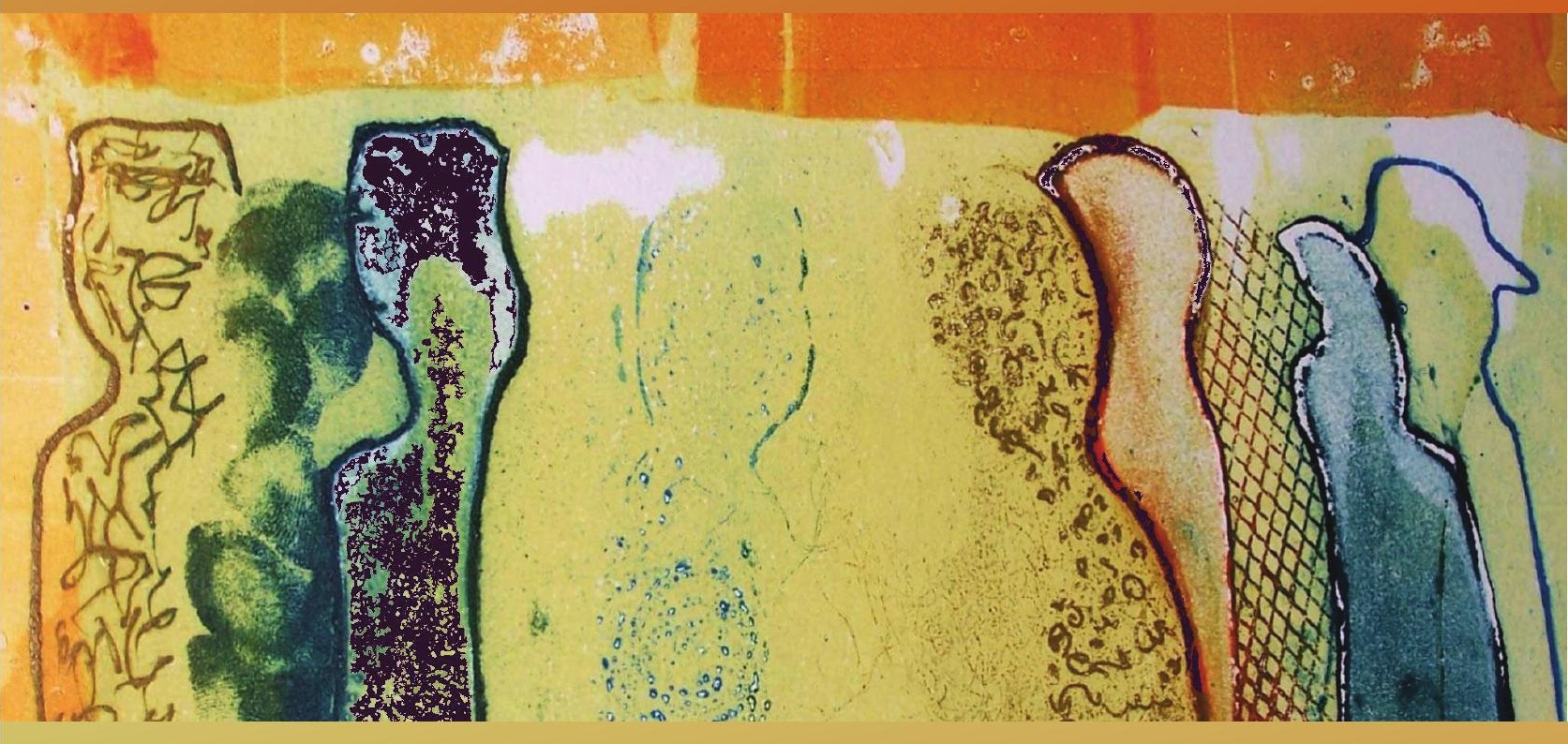

\title{
On the generation of shared symbols
}

\section{DONDERS}

\section{series}


On the generation of shared symbols

Arjen Stolk 


\title{
On the generation of shared symbols
}

\author{
Proefschrift
}

ter verkrijging van de graad van doctor aan de Radboud Universiteit Nijmegen op gezag van de rector magnificus prof. mr. S.C.J.J. Kortmann, volgens besluit van het college van decanen

in het openbaar te verdedigen op dinsdag 2 september 2014

om 12.30 uur precies

door

\section{Arjen Stolk}

geboren op 31 oktober 1982

Ph.D. thesis, Radboud University Nijmegen

te Arnhem

On the generation of shared symbols

C 2014 Arjen Stolk

ISBN 978-94-62592-52-0

Cover design: In gesprek, Marianne Schipper 


\section{Promotor}

Prof. dr. I. Toni

Copromotoren

Dr. ir. R. Oostenveld

Dr. L. Verhagen

\section{Manuscriptcommissie}

Prof. dr. O. Jensen

Prof. dr. J.J.A. van Berkum (Utrecht University)

Prof. dr. R.T. Knight, MD (University of California, Berkeley)

\section{Table of Contents}

Chapter 1 General introduction 6

Chapter 2 Analytical tools for studying social neural dynamics

2.1 Online and offline tools for head movement compensation in MEG

Chapter 3 Social neural dynamics of human communication

3.1 Neural mechanisms of communicative innovation

3.2 Cerebral coherence between communicators marks the emergence of meaning

Chapter 4 Interfering with human communication

4.1 Understanding communicative actions: a repetitive TMS study

4.2 Altered communicative adjustments following ventromedial prefrontal lesions

Chapter 5

Development of human communication

5.1 Early social experience predicts referential communicative adjustments in five-year-old children

Chapter 6 General discussion

24

Appendix

Dutch summar

Reference list

Donders series

Acknowledgments

About the author

Publication list 

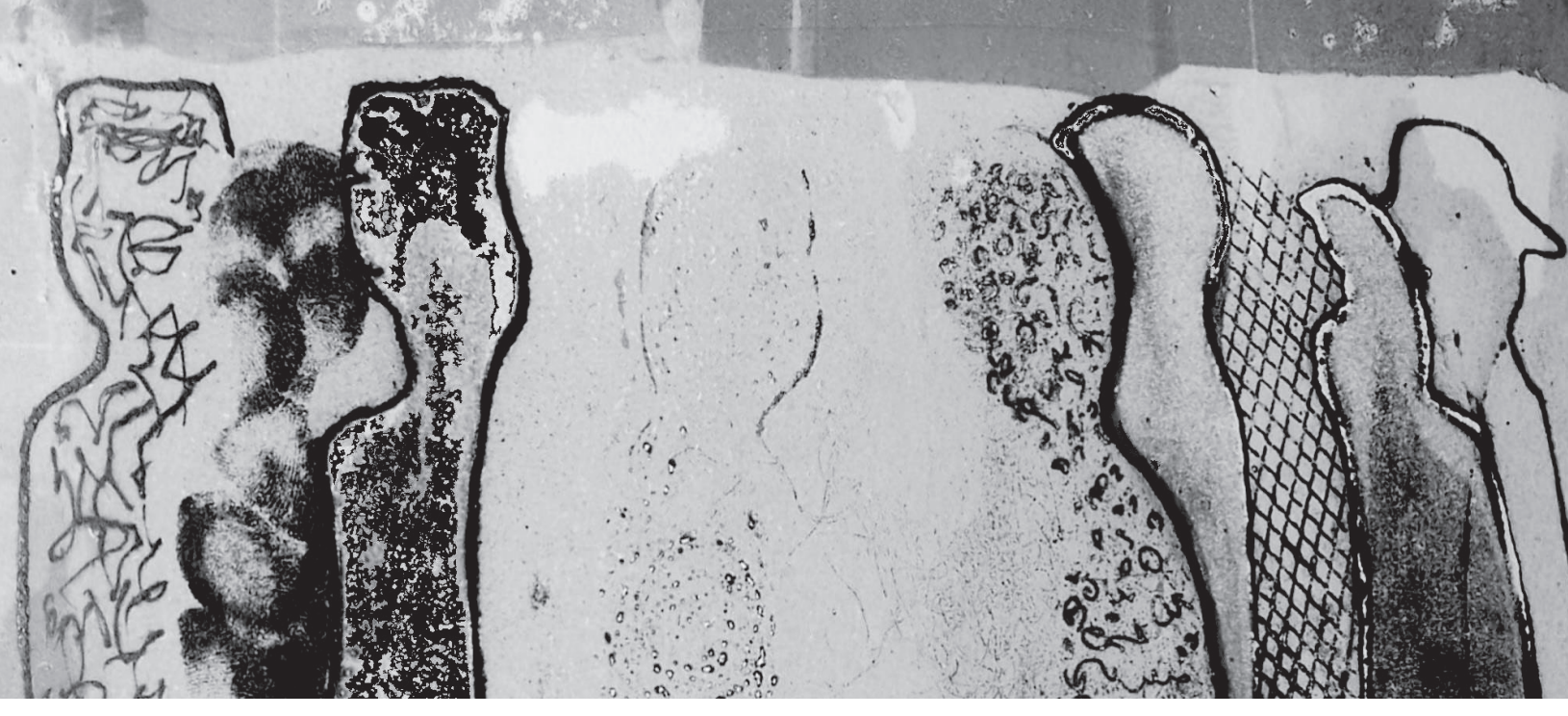

General introduction 
Imagine yourself in a bar grasping an empty glass from a table and raising it in the air. To understand this action, it is relevant to study the mechanisms that mediate the grasping and raising movement, for instance how retinal patterns of photic stimulation can be translated into muscular patterns of torques. This explanatory framework is fundamental to account for the instrumental aspects of the movement, but a bartender observing the glass-raising action would sorely miss its point by considering only its sensorimotor features. Some actions, perhaps most human actions, are selected and comprehended by taking into account not only their mechanical consequences, but also their social implications. The challenge is to specify how the social constructs that disambiguate communicative actions are mechanistically integrated with sensorimotor processes. The goal is to generate a neurobiologicallygrounded account of the computational mechanisms supporting our referentia communicative abilities, a necessary step to understand the biological and cognitive bases of human sociality. This thesis is concerned with understanding our ability to select actions with the intent of altering another agent's mental state, as epitomized by the foresight that leads a customer to order a drink by raising his empty glass in front of a bartender. 
Referential communication is a complex and anomalous instance of biological social interactions (Dawkins \& Krebs, 1978; Owings \& Morton, 1998). Referential communication is anomalous because it relies on context-dependent behaviors designed to influence the mental state of specific addressees, rather than on stable traits designed by natural selection to reliably influence bystanders (Danchin, Giraldeau, Valone, \& Wagner, 2004). Referential communication is complex because each of its behavioral vehicles can carry multiple meanings, and a given meaning can be conveyed by a variety of behaviors. A great deal of effort has been spent in understanding features and rules of the system most frequently used by humans for achieving referential communication, i.e. natural language (Chomsky, 1995; de Saussure, 1910-1911; Jackendoff, 2002). Although those efforts have undoubtedly improved our understanding of the cognitive structures intrinsic to the language faculty (Hauser, Chomsky, \& Fitch, 2002), considerably less emphasis has been given to defining the cognitive processes that support the communicative use of language (Levinson, 2006; Schilbach et al., 2013; Wittgenstein, 1953/2001). This thesis steps into this gap by focusing on our ability to share the meaning of a novel symbol, independently from the conventions and additional complexities introduced by linguistic processing (de Ruiter et al., 2010; Galantucci \& Garrod, 2011). Although it is often assumed that pre-existing symbols can be shared across interlocutors by simply coding and decoding them, using those symbols requires a computational mechanism powerful enough to mutually negotiate them across communicators (Levinson, 2006). Studying the generation of novel shared symbols provides a privileged window into this mechanism: given that novel symbols lack a pre-existing shared representation, jointly establishing their meaning relies on converging on a common ground of knowledge and beliefs across communicators, even more so than the meaning of already known words and gestures. As elaborated in the next section, existing accounts cannot explain the exceptional flexibility of human referential communication (when compared to other forms of animal communication), which may underlie our ability to share meanings and create language in the first place (Levinson, 2006). This thesis elaborates on the neural mechanisms supporting this human faculty, addressing the question of how communicators can design and interpret communicative acts. Starting from the premise that the generation of shared symbols depends on inferred knowledge and beliefs of a communicative partner, i.e. conceptual knowledge that accumulates and is sharpened in our minds as we interact, I reason that these mechanisms should be shared by the interlocutors of the communicative exchange and involve conceptual predictions based on a dynamic conversational context.

\section{Existing accounts of human communication}

In the late 40s, communication was formalized by Claude Shannon as an instance of signal transmission (Shannon, 1948). In Shannon's framework, agents can communicate as long as they have the same set of pre-defined coding-decoding rules. However, that framework does not explain how agents can negotiate those rules. Natural selection can drive organisms towards shared coding-decoding rules across multiple generations (Danchin, et al., 2004), bu this account does not explain how humans can rapidly disambiguate situations lacking pre-defined coding-decoding rules. This is not an exceptional situation. In fact, we achieve this feat during most daily conversations, when learning a language as infants, or when communicating with others in the absence of a common idiom (Levinson, 2006; Noordzij et al., 2010). Even common words do not contain fixed meanings - they may provide us with clues on a communicative meaning - but are coordinated through an interactive process by which people in dialogue seek and provide evidence that they understand one another (Brennan, Galati, \& Kuhlen, 2010). For instance, when a customer asks a bartender "Could you prepare a Margarita?", the bartender is not likely to pause wondering why the customer is questioning his skills, and the customer would not be puzzled by a logically un-related answer like "Happy Hour starts in five minutes".

Studies on natural dialogue and recent reports in controlled experimental situations (de Ruiter, et al., 2010; Galantucci, 2005; Scott-Phillips, Kirby, \& Ritchie, 2009) have shown that humans quickly develop new semiotic conventions when they need to. However, it remains to be explained how those new semiotic conventions can be generated in the absence of an a priori common code. Computer simulations, using reinforcement-learning algorithms, have shown that communication systems can arise without the presence of common knowledge (Barr, 2004; Kirby \& Hurford, 2002; Puglisi, Baronchelli, \& Loreto, 2008; Steels, 2003). For instance, two computer agents can share novel symbols by virtue of guesses and explicit performance feedback (Steels, 2003). However, establishing these arbitrary signal-meaning mappings required many thousands of pair-wise interactions. Accordingly, general-purpose learning algorithms like temporal difference (Behrens, Hunt, \& Rushworth, 2009) or Hebbian learning (Keysers \& Perrett, 2004) do not seem suitable to explain the human ability to quickly grasp a meaning or to design an action that can be understood from scratch (de Ruiter, et al., 2010) since those learning algorithms require many trials to converge on statistically relevant features. Other scholars have suggested that human referential communication relies on cognitive modules that are involved only when communication requires it, e.g. when having to "repair" a misunderstanding (Horton \& Keysar, 1996; Keysar \& 
Horton, 1998) or when a certain representation is primed (automatically) by the utterance of an interlocutor (Garrod \& Pickering, 2004). Further simplifications of this approach has led other scholars to suggest that actions can convey communicative meanings "without any cognitive mediation", by virtue of an automatic sensorimotor mechanism ("mirroring") that link the mental representation of an observed action to the representation of an executed action, and the latter to its outcome (Rizzolatti \& Craighero, 2004). However, those accounts leave un-specified how humans can effectively repair, prime, or "mirror" a communicative action when required, and they remain silent on how we organize our behavior for conveying intentions. Automatic priming, reinforcement learning, or sensorimotor associations might be instrumental in finessing a solution once a communicative action has been drafted, but they do not seem suitable to explain how we can rapidly converge towards a common understanding of a novel symbol. Those symbols, being novel, do not have well-defined priors (Fodor, 2000; Levinson, 2006; Sperber \& Wilson, 2001) and dedicated neuronal circuits for unpacking their references (Giese \& Poggio, 2003; Peelen, Fei-Fei, \& Kastner, 2009).

Accordingly, the generation of shared symbols requires a mechanism that allows us to rapidly converge on a shared meaning, constraining a potentially infinite cognitive search space of mappings between symbols and representations. I suggest that this mechanism should be shared by interlocutors of the communicative exchange and, in order to alter an interlocutor's mental state in a predictable manner, should involve predictions based on presumed knowledge and beliefs of that specific interlocutor, conceptual knowledge that needs to be continuously updated and sharpened according to the shared history of the interaction. This account is closely linked to accounts of human social abilities based on the theory-of-mind framework (Frith \& Frith, 2006; Premack $\&$ Woodruff, 1978). In this framework, the assumption is that behavior is the observable product of mental states, and making inferences about these mental states ("mentalizing" (Grezes, Frith, \& Passingham, 2004)) requires knowledge of their content and relationship with behavioral responses (Nichols \& Stich, 2003). This conceptually-based account of our mentalizing abilities has been linked with cerebral structures that are distinct from the sensorimotor system, and include the superior temporal sulcus, the temporo-parietal junction, the temporal poles, and the medial prefrontal cortex (Amodio \& Frith, 2006; Frith \& Frith, 1999, 2006; Grezes, et al., 2004; Walter et al., 2004). Unfortunately, the majority of imaging studies that aimed to probe this theory-of-mind network have been conducted in non-interactive settings. In those settings, participants read or view story scenarios that trigger reasoning about the mental state of story characters (Saxe, Xiao, Kovacs, Perrett, \& Kanwisher, 2004). To date, the exact roles and contributions of the distinct brain regions making up the theory-of-mind network remain unknown. Furthermore, the theory-of-mind framework is theoretically heterogeneous (Carruthers, 1996; Leslie, Friedman, \& German, 2004; Nichols \& Stich, 2003) and it remains to be seen whether and how theory-of-mind mechanisms play a role in genuine social interaction (Schilbach, et al., 2013).

\section{Novel shared symbols as a privileged window into} communicative interactions

The central tenet of this thesis is that the study of novel shared symbols provides a privileged view into the mechanisms of human communication, capturing the joint construction of meaning across interacting agents, contingent on the interaction dynamics, rather than on stereotypical stimulus-response mappings (Hasson, Ghazanfar, Galantucci, Garrod, \& Keysers, 2012; Rizzolatti \& Craighero, 2004). Unveiling the fundamental properties of human referential communication requires experimental procedures that capture these principles of human interaction, rather than surface features (e.g. conventional linguistic representations). One way to address this issue is to generate experimental situations in which people need to communicate independently from the speech and gestures that are often used as behavioral vehicles for those mental representations. Novel symbols, like new words and gestures, are arbitrary tokens that may represent and be used to convey ideas and beliefs while their meaning becomes shared between interlocutors. Studying how people generate shared novel symbols (technically known as 'experimental semiotics', (Galantucci \& Garrod, 2011)) therefore may provide a window into the mechanisms supporting the human competence to rapidly generate and understand communicative actions.

An experimental platform suitable for studying human communicative interaction requires that it is simple enough to be abstracted in computationa models and neurophysiological experiments. Yet, it needs to be sufficiently flexible to capture non-trivial aspects of human communication. Several human communicative games have been developed and studied (Camerer, 2003; Feiler \& Camerer, 2010; Galantucci, 2005; Scott-Phillips, et al., 2009; Selten \& Warglien, 2007), with the 'Tacit Communication Game' (de Ruiter, et al., 2010) being one of the few that has been studied from both a computational and neuroscientific perspective. In this communication game, interlocutors do not have access to pre-existing conventions (e.g. a common language, body emblems, facial expressions) that may provide clues on the meaning of a symbol. The only available communicative vehicle consists of geometric shape movements, controlled by and visible to both players on a game board. This 
novel medium enforces the participant pairs to mutually (re-)negotiate novel symbols over the course of the task, effectively creating a new communication system. Consequently, the same symbol can be used by different communicative pairs to negotiate different meanings. The same symbol can even be used to convey different meanings by the same pair at different points in time, and vice versa (for examples see movies in (Stolk et al., 2013)). These behavioral observations emphasize how, in this task, a symbol acquires meaning by virtue of the history of the communicative interactions within a given pair, rather than by virtue of its sensory attributes.

The goal of the communication game is for pairs of participants - as a Communicator and an Addressee - to jointly re-create a spatial configuration of two geometric shapes shown only to the Communicator (see the thought cloud in Figure A of BOX 1, and epoch 2 in Figure B). This requires the Communicator to use the movements of his shape (in blue, epoch 3 in Figure B, BOX 1) to indicate to the Addressee how she should configure her shape (in orange). There are no a priori correct solutions to this communicative task, nor a limited set of options from which the Communicator can choose. The Addressee cannot solve the communicative task by reproducing the movements of the Communicator's shape. Rather, she needs to disambiguate communicative and instrumental components of the Communicator's movements, and find some relationship between the shape movements, i.e. the symbol, and their meaning. Success in this game thus relies on the Communicator designing a symbol that can be understood by the Addressee, and on the Addressee inferring the Communicator's intentions. Participants turn out to be remarkably successful communicators under these constrained conditions (de Ruiter, et al., 2010). Given that they do not have access to pre-existing conventions, the participant pairs need to take into account the presumed beliefs and knowledge of their interlocutors when selecting and interpreting novel symbols as Communicators and Addressees respectively. Manipulation of the task structure shows that game performance (i.e. number of spatial configurations successfully recreated by the two players) improves when Communicators are able to see the Addressees' behaviors (epoch 5 in Figure B), suggesting that they take into account how Addressees interpreted their messages (de Ruiter, et al., 2010). This interpretation is reinforced by another study (Blokpoel et al., 2012) showing that changes in the Communicators' movement characteristics after a misinterpretation of the Addressees are dependent on the type of error made by the Addressee. If an Addressee had placed her shape in an incorrect location, but with correct orientation, the Communicator tended to pause relatively longer on the Addressee's goal location. Such change in behavior is intended to indicate that a long pause should be interpreted as being dissociated from the rest of the movement, making it in effect less ambiguous for the Addressee which of the locations on the board was marked by the Communicator as the Addressee's goal location.

In sum, the communication game induces the generation of symbols that pertain to the inferred knowledge of the communicative partner. Within this task, communicative difficulty is easy to manipulate, using different combinations of shapes (for examples see (Blokpoel, et al., 2012)). Furthermore, it allows manipulating common ground knowledge across communicators, by having pairs encounter problems for which they previously had jointly established a solution. In this thesis, I discuss empirical findings obtained in the context of this interactive task that throw light on the fundamental properties of human referential communication. 


\section{BOX 1. The 'Tacit Communication Game'}

The emergence of shared symbols as a privileged window into communicative interactions

The Tacit Communication Game involves two players, a Communicator and an Addressee (left and right person respectively in the scenario depicted in Figure A), controlling geometric shape movements on a game board. Their joint task is to re-create a spatial goal configuration of two eometric shapes. The crucial manipulation is that this target information is known to one of the players only - the Communicator - who then needs to convince the other player - the Addressee - to move her shape (in orange) to the desired target location and orientation The game is tacit: the two players interact only through the visual consequences of their movements on the game board. The Communicator can thus only convey a message to the Addressee by moving his shape (in blue) knowing that she will observe those movements to decide where and how to move her shape. For experimental purposes, the game setup is computer-programmed and presented individually on two separate monitors (Figure B). The players control their shape movements (horizontal and vertical translations, and 90 degree

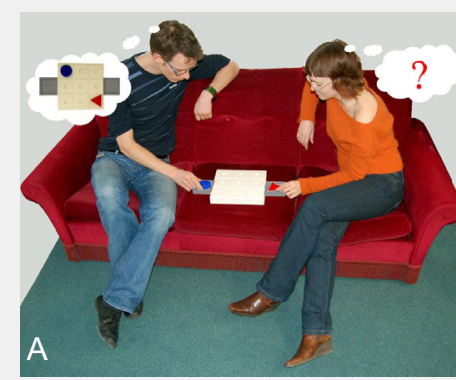
(lockwise rotations) using hand-held controllers. At to each player (event 1 in the same figure), followed by presentation of the goal configuration to the Communicator (event 2). During this event, he can plan as long as needed, but he has only five seconds to execute his movements in the next. After pressin a start button, the Communicator's shape will appear in the center of the grid. He now can execute his actions, visible to the Addressee who needs to infer

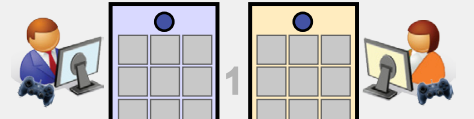
the Communicator's intentions from his movements (event 3). For instance, by first going to her target location, ostensibly 'pause' to indicate the relevance of that location (number 1 action), and then 'wiggle' to indicate her shape's orientation (number 2 action), and then completing his own target configuration (number 3 action). After this event, the Addressee can plan (event 4) and execute her actions (event 5) in order to complete their joint goal configuration. Finally, the same feedback on their task performance is presented to both players in the form of a green tick or a red cross (event 6). Please note that this is only one among a series of possible solutions. For instance, some participants converge on using the number of subsequent 'wiggles' to mark the number of clockwise rotations that the Addressee needs to make to achieve the target orientation of her shape, while others do not use the 'wiggle' but leave the triangle location along the direction to which the triangle needs to point.
Outline of the thesis

This thesis is concerned with understanding our remarkable ability to select actions with the intent of altering another agent's mental state, as epitomized by the foresight that leads a customer to order a drink by raising his empty glass in front of a bartender. This thesis contributes to understanding human communicative abilities by focusing on the generation of novel shared symbols. Novel symbols, like words and gestures, are used to convey ideas and beliefs, and their meaning emerges as those symbols become shared across interlocutors. Here, I present an outline of the studies described into detail in the following chapters.

Studying social neural dynamics comes with hard methodological challenges. First, it is necessary to appropriately capture the social dynamics. That means having an experimental platform that- combines experimental control with sensitivity to the multidimensional and time-varying features of interpersonal interactions (BOX 1). Second, it is necessary to appropriately capture the neural dynamics. Magnetoencephalography (MEG) offers the possibility to do that, non-invasively, in humans (BOX 2). However, MEG is also exquisitely sensitive to variations in the position of the head of participants engaged in communicative interactions over multiple experimental sessions. Chapter 2.1 describes a set of tools that I have created to overcome those methodological issues, a necessary step for being able to capture functionally specific neuronal markers of human communicative interactions.

In this thesis, human communication is considered a joint construct of interacting agents, contingent on the interaction dynamics. Meanings are continuously negotiated during most daily conversations, when learning a language as infants, or when communicating with others in the absence of a common idiom. Even common words do not contain fixed meanings - they may provide us with clues on a communicative meaning - but are coordinated through an interactive process by which people in dialogue seek and provide evidence that they understand one another. Chapter 3 captures the social neural dynamics of the mechanisms supporting our referentia communicative abilities, as operationalized with the communication game outlined in BOX 1. In chapter 3.1, I use MEG to assess spectral, temporal, and spatial characteristics of neural activity evoked when people generate and understand novel shared symbols during live communicative interactions. By directly contrasting those phenomena with a control interaction involving no communicative necessities, I test the prediction that both production and comprehension of novel communicative actions rely on shared neural patterns associated with flexible conceptual knowledge. In chapter 3.2 I qualify the 
characteristics of this flexible conceptual knowledge, showing that it relates to inferred knowledge and beliefs shared across interlocutors. I simultaneously record fMRI (BOX 2) in two interacting participants engaged in building a pair-specific conversational context during the communicative exchange. I use an experimental manipulation of common ground dynamics to isolate patterns of cerebral activity shared across interlocutors. I isolate cerebral signals with stronger coherence across communicating pairs than across participants engaged in performance of the same task, but without a shared common ground dynamics. This section shows that updating of communicative ground relies on inferential mechanisms shared across communicators, contingent on the ongoing interaction.

The studies in chapter 3, as well as many others dealing with understanding of another agent's intentions, have highlighted several key cortical regions, such as the right posterior superior temporal sulcus (pSTS) and the ventromedial prefrontal cortex (vmPFC). Chapter 4 qualifies the contributions of those regions to human communicative abilities by interfering with their neural activity. In chapter 4.1 I disturb neural activity in right pSTS to test whether this region is necessary for understanding the meaning of novel communicative actions. Specificity of TMS intervention (BOX 2) is ensured by controlling for the communicative relevance of the stimuli and for the cerebral location of the intervention. In chapter 4.2, I exploit an unfortunate experiment of nature and test whether a lesion in the vmPFC impairs patients' ability to adapt their referential communicative behavior to the presumed age, or cognitive level, of their interlocutor. Specificity of the effects of brain damage is ensured by lesionsymptom mapping and by comparing communicative behaviors evoked by vmPFC patients with behaviors evoked in lesion- and healthy-control subjects.

In chapter 5 I consider a different element of the causal mechanisms supporting our communicative abilities. I take a developmental perspective to understand how the neural mechanisms isolated in chapters 3 and 4 are acquired and shaped. Building on the suggestion that collaborative experiences early in life might be crucial for the emergence of mental coordination abilities, in chapter 5.1 I assess the relative contribution of social exposure to familial and non-familial agents on 5-year-olds' referential communicative adjustments to their mental model of an addressee within a communication game. In contrast to linguistic communication, the communicative behaviors evoked under these experimental conditions cannot be directly based on previous concrete experiences. Accordingly, the novel situation that is experienced by the children allows me to directly tap into their ability to influence the mental states of others through internally generated behaviors.
Lastly, chapter 6 provides an integrated view of the mechanisms supporting our communicative abilities, in the light of the novel findings described in this thesis. It provides a general outlook on how this view influences our understanding of the ability to select actions with the intent of altering another agent's mental state and proposes possible directions of future research. 


\section{BOX 2. Cognitive neuroimaging methods}

Convergent tools to investigate the neural mechanisms supporting communicative interactions

\section{Magnetoencephalography (MEG)}

This technique measures the magnetic fields arising from electrophysiological activity in the brain. These magnetic fields are very small - approximately one millionth of the earth's magnetic field - and require superconductive magnetic field sensors ('SQUIDS') and a magnetically shielded room in order to be measured. In contrast to single-cell recordings which measure predominantly the neuronal output of a neuron (i.e. an 'action potential', $1 \mathrm{~ms})$, MEG measures local field potentials generated by many neurons. These potentials are mainly determined by gradual fluctuations in neuron membrane potentials, relatively sluggish compared to the action potentials, caused by the input received from many other neurons (see Appendix B of chapter 3.1 for more details). If a large enough assembly of neurons with dendrites is sufficiently aligned, as it is often the case for large pyramidal neurons in the human brain cortex, many simultaneous inputs to that assembly will spatially and temporally add to each other. This integrated signal may lead to an extracellular current strong enough to be measured outside the head. In combination with its high temporal resolution - at the rate of 1 millisecond - the instantaneous detectability of these neurophysiologic processes provides MEG with high temporal precision. Furthermore, MEG signals have spectro-temporal characteristics (i.e. frequency, amplitude, and phase of a cortical rhythm) that reflect different physiological mechanisms and cognitive functions (see Appendix B of chapter 3.1). In this thesis I have exploited this property of MEG to implement a stringent test of the hypothesized computational overlap between selection and comprehension of a communicative action (chapter 3.1).

Studying social neural dynamics with MEG requires additional experimental and analytical tools. The subject's head, and thereby the neuronal sources in the brain generating the neuromagnetic fields, can move relative to the MEG sensors. Head movement, and consequentially different head positions, has detrimental effects on the statistical sensitivity of MEG data (see chapter 2.1). Given the need for recording the communicative and control interactions over two sessions as each interactive task requires different instructions and practice sessions, I developed an online experimental procedure that allows for optimal repositioning of subjects' heads between sessions, promoting inter-session consistency in head location (procedure described in chapter 2.1). Following, an absolute index of neural activity could be computed (chapter 3.1), i.e. source-reconstructed time-resolved estimates of signal frequency power, using adaptive spatial filtering of neural activity recorded at the MEG sensors. This index is appropriate for isolating state-dependent effects that are not exclusively bound to the occurrence of task events, as one could expect with neural correlates of communicative signals whose meanings depend on the communicative context.

\section{Functional magnetic resonance imaging (fMRI)}

In magnetic resonance imaging (MRI), a strong static magnetic field is used to bias the alignment of the magnetic dipoles of hydrogen atoms in the body. Radio-frequency pulses, in combination with variable magnetic gradient fields perpendicular to the static magnetic field, selectively excite particular body areas (in our case the head) by temporarily flipping the magnetization spin axis of the local hydrogen atoms out of alignment with the static magnetic field. The precessing nuclei induce a voltage in a receiver coil placed around the body region of interest. This technique allows for constructing structural images of the brain with different tissue types in the brain (e.g. grey and white matter, cerebral spinal fluid, skull, and fat) exhibit different MR signals. Functional MRI (fMRI) takes advantage of the different magnetic properties associated with oxygen-rich and oxygen-poor blood. These properties relate to the levels of oxygenation of the oxygen-transporting protein haemoglobin, abundant in red blood cells. These levels can be quantified as the blood-oxygenated-level-dependent signal (BOLD), a signal that can be measured accurately with high spatial resolution, currently on the volumelevel of $1 \mathrm{~mm}$ cubed, known as a volumetric pixel, or 'voxel'. The BOLD signal is generally considered a good proxy for neural activation. Neurons require energy to function and take up glucose and oxygen from the blood in surrounding capillaries. When a neuronal population becomes more activated, it will consume more oxygen. To replenish the consumed oxygen, blood flow and volume is locally increased, potentially a consequence of the dilation of smooth capillary muscles which might be initiated by a signal cascade involving the release of diffuse nitric oxide by the neurons themselves and other chemical signals from nearby supporting glial cells. This 'haemodynamic response', reflected in the BOLD signal, is sluggish and peaks about 6 seconds after neuronal activation and may take more than 20 seconds to return to baseline. Despite this delay and its low temporal resolution - an inter-scan-interval typically lasts 2 seconds or longer - fMRI allows for accurate mapping of human brain function. Its high spatial resolution offers the opportunity to localize differential neural activity in regions with high precision.

As outlined in chapter 3.2, I hypothesize that conveying and understanding the meaning of signal relies on inferred common ground knowledge, contingent on the ongoing communicative interaction. To test this hypothesis I simultaneously monitored cerebral activity (dual-fMRI) in both elements of communicating pairs and localized changes in BOLD signal with stronger coherence within communicative pairs than across the group of participants engaged in task performance.

\section{Transcranial magnetic stimulation (TMS)}

This technique uses electromagnetic induction, thereby overcoming the electrical insulation of the skull, to evoke low-intensity electrical currents targeted to the surface of the cortex. During TMS, a very rapidly changing electric pulse is passed through a conductive coil held above the head. This changing current generates a pulsed magnetic field - following Faraday's law - that penetrates the scalp and skull reaching the neural tissue of the cerebral cortex underneath. Depending on conditions like current direction and orientation, stimulus strength, pulse shape, and importantly, the neural state at the moment of stimulation, the electromagnetically induced electric current can excite neurons, triggering action potentials, but also modulate neuronal excitability inducing facilitated or inhibited states. Interestingly, these modulatory effects can even outlast the time of stimulation when a repetitive stimulation protocol is used. For example, repetitive stimulation at $1 \mathrm{~Hz}$ for about 20 minutes has shown to reduce metabolic activity and cortical excitability within the stimulated area by $5-30 \%$ for up to 30 minutes. 
The transient perturbation of a brain region allows for testing of the necessity of that region for a particular function (e.g. the role of posterior superior temporal sulcus in understanding communicative actions, chapter 4.1). This technique thereby allows moving beyond associations of brain activity with behavior, correlational evidence that cannot rule out epiphenomena. Advantages of an offline repetitive stimulation protocol, as I have applied, as compared to an online protocol in which disruptive stimulation takes place during task performance, are that temporally stable and task-independent modulation of local cortical processing efficiency can be induced and that non-specific distracting effects of the auditory and somatosensory sensation can be avoided. 

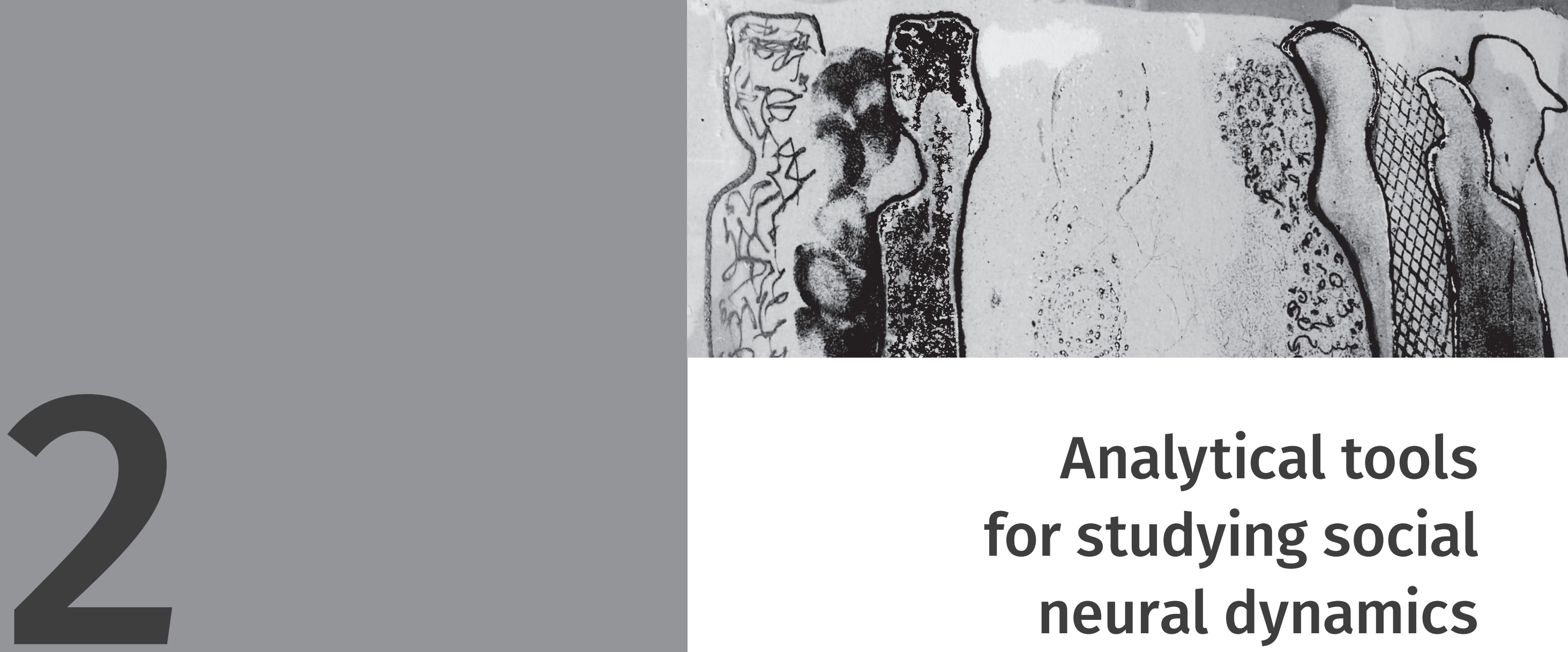

\author{
Analytical tools \\ for studying social \\ neural dynamics
}




\section{Online and offline tools for head movement compensation in MEG}

Stolk A, Todorovic A, Schoffelen JM, Oostenveld R 


\section{Introduction}

MEG measurements are performed with superconductive magnetic field sensors that are mounted inside a helmet-shaped dewar. The subject's head is positioned under the dewar as close as possible to the sensors, but in most MEG experiment settings the subject's head is not fixated. The consequence is that the subject's head, and thereby the neuronal sources in the brain generating the neuromagnetic fields, can move relative to the MEG sensors. To minimize the detrimental effects of head movements on the data, subjects are typically instructed by the experimenter to maintain the same posture and lay or sit still throughout the recording, which can take a considerable amount of time.

Head movement during recording causes topographical blurring of the measurements at the sensor level and thereby introduces source localization errors (Medvedovsky, Taulu, Bikmullina, \& Paetau, 2007; Uutela, Taulu, \& Hamalainen, 2001). Also, the mixture of different head positions over time adds variance to the data that is not accounted for by the experimental manipulation, thus potentially deteriorating statistical sensitivity. Studies that involve subjects who have difficulty remaining still (Gaetz, Otsubo, \& Pang, 2008; Wehner, Hamalainen, Mody, \& Ahlfors, 2008) or studies that involve recordings of the same subject in multiple sessions (potentially over multiple days) (Nieuwenhuis, Takashima, Oostenveld, Fernandez, \& Jensen, 2008), face a related problem. A difference in the head position causes not only the MEG sensor level topographies to be inconsistent between the sessions, but may also lead to differences in the signal-to-noise ratio between sessions, due to the weaker signal that is picked up from a source when it is further away. Without accurate repositioning over sessions, the comparison or the combination of data from separately recorded sessions suffers from the increased between-sessions variance.

Ideally, one would allow the MEG subjects to move freely and correct the sensor level data for the movements. In EEG recordings, head movements are in principle not problematic because the electrodes move along with the head. For fMRI it is possible to compensate for movements during the acquisition by instantaneous adjustments of the gradients (Maclaren, Herbst, Speck, \& Zaitsev, 2012; Thesen, Heid, Mueller, \& Schad, 2000). Future advances with optically pumped magnetometers (Kominis, Kornack, Allred, \& Romalis, 2003; Sander et al., 2012) may result in a cap-style MEG sensor array that can move along with the head. At present, MEG recordings have to be performed with a spatially fixed sensor array, relative to which the head can move. Offline compensation methods are available to correct the raw sensor level data (Knosche, 2002; 
Numminen, Ahlfors, Ilmoniemi, Montonen, \& Nenonen, 1995; Taulu, Simola, \& Kajola, 2005; Uutela, et al., 2001), which attempt to approximate the MEG data that would have been recorded, had the head been on a fixed position relative to the sensors. The compensation techniques rely on model assumptions, such as stationarity of the signal-to-noise ratio and the field distributions. However, after correction the MEG data does not correspond ideally with the desired data due to the imperfection of the assumptions and the difficulty for the user selecting the optimal algorithm parameters.

Preferably, head position differences and movements are avoided in the first place, e.g. by fixating the subject's head using a subject specific nylon or silicon head-cast (Gareth Barnes, personal communication). Bite-bars have been employed in MEG for the purpose of accurate co-registration with MRI (Adjamian et al., 2004; Singh, Holliday, Furlong, \& Harding, 1997) but have not been used to fixate the head relative to the MEG sensors. For magnetic resonance imaging bite-bars have sporadically been used to reduce head movements (e.g. (Heim, Amunts, Mohlberg, Wilms, \& Friederici, 2006)). However, a limiting factor in the applicability of a bite-bar in MEG recordings is increased muscle tension in the jaw muscles and the associated increase in noise.

Besides implementing strategies to avoid head movements, methodological advances in the past decade have been proposed to compensate for the effects of head movements in offline data analysis. For this purpose, most MEG systems are able to continuously localize the position of the head relative to the dewar (de Munck, Verbunt, Van't Ent, \& Van Dijk, 2001; Uutela, et al., 2001; Wilson, 2004). Yet, the majority of published MEG studies to date do not incorporate this knowledge about head movements in the analysis pipelines. The present study explores the potential of using continuous head location monitoring for online and offline head movement compensation with a focus on the consequences of head movements on statistical sensitivity. Without downplaying the relevance of obtaining crisp MEG sensor-level topographies and accurate source localizations, most cognitive research questions are addressed using an experimental manipulation to statistically test a hypothesis. Consequently, the effects of head movements have impact on the usability and sensitivity of MEG in the study of cognition.

The remainder of the introduction is structured as follows: We will first provide a common methodological framework that describes the problem of variability in head position. Subsequently, we will present each of the existing methods suggested in the literature in this common framework and explain their opportunities and limitations. Finally, we will introduce our methodological contributions and how we will assess their potential for improving statistical sensitivity.

For a given source $\mathbf{S}$ with location $r$ and time sample $t$, the data $\mathbf{X}$ at MEG sensors can be represented as the magnetic field of that source projected through lead field $L$ (i.e. the physical forward model of the field distribution):

$$
\mathbf{X}(t)=\mathbf{L}(r) \cdot \mathbf{S}(r, t)+\mathbf{N}(t)
$$

where $\mathbf{N}$ is measurement noise. The dimensions of matrix $\mathbf{X}$ and $\mathbf{N}$ are channels by time samples, the dimensions of matrix $\mathbf{L}$ are channels by number of source components (e.g. three orientations for a free-orientation dipole), and the dimensions of matrix $\mathbf{S}$ are number of source components by time samples. Rather than expressing the position of the head relative to the sensors, we consider the position of the MEG sensors relative to the head and the location $r$ of the source is also expressed relative to the head. If we now consider that the MEG sensors move relatively to the head, the data can be described as

$$
\mathbf{X}(t)=\mathbf{L}\left(r_{s} ; r_{h}\right) \cdot \mathbf{S}\left(r_{s}, t\right)+\mathbf{N}(t)
$$

where $r_{s}$ is the source location and $r_{h}$ is the helmet location, i.e. the position of the helmet-shaped sensor array relative to the head. When $r_{h}$ is not constant over time samples $t$, such as after head movements within a recording session or after the concatenation of separate sessions, this will introduce variability over time of $\mathbf{L}$ and thereby of the signal in data $\mathbf{X}$. In terms of MEG experiments involving repeated trials of an evoked or induced brain response, different head positions will cause trial-by-trial variance that is not accounted for by the description of the experimental manipulation. Consequently, this leads to blurred topographies, increased localization errors, and reduced statistical sensitivity.

The existing offline approaches that try to compensate for head movements can broadly be divided into two categories. One category accounts for head position differences and movements at the sensor level and the other category at the source level. The sensor level correction is based on the interpolation/ extrapolation, or realignment, of the magnetic field distribution measured by the sensor array to a magnetic field distribution that would have been measured had the sensors been at the desired location. This interpolation can be achieved by an inverse modeling step, based on a distributed source model and using the original sensor positions, followed by a forward modeling step, where the reconstructed source activation is projected to a new set of sensor positions (Knosche, 2002; Numminen, et al., 1995) (see Eq. (7)). Typically, the 
inverse model involves a regularized minimum norm estimate, using a large number of dipoles that are placed near the surface of the brain compartment. Another approach is signal space separation (SSS), which allows for a sensor level interpolation that is based on modeling the magnetic field distribution using a set of spherical harmonic functions (Medvedovsky, et al., 2007; Taulu, et al., 2005). The model can subsequently be used to estimate the magnetic field distribution at the desired sensor positions. This technique is implemented in the 'MaxFilter' software (Elekta Neuromag Oy, Helsinki, Finland).

Alternatively, head position information can be incorporated into the source reconstruction procedure. Source reconstruction involves the construction of an inverse linear operator, that can be thought of as a (pseudo-) inverse of the lead field, $\mathbf{L}^{+}$(Dale \& Sereno, 1993). Typically, the lead field matrix and its inverse are considered to be time-invariant, assuming a fixed position of the neuronal source model with respect to the sensors. Variability of the positions of the MEG sensors relative to the head can be taken into account by adjusting the lead field gains that relate the source amplitudes to the field distribution (Uutela, et al., 2001). Correspondingly, the estimated source activity $\hat{\mathbf{S}}$ can be expressed as:

$$
\hat{\mathbf{S}}\left(r_{s}, t\right)=\mathbf{L}^{+}\left(r_{s} ; r_{h}\right) \cdot \mathbf{X}(t)
$$

where $r_{h}$ reflects the position of the MEG sensors relative to the head. The lead field matrix can be adjusted on a trial-by-trial basis, i.e. by using a separate lead field for each trial/repetition $k$ corresponding to the head position during that trial, and by averaging afterwards:

$$
\hat{S}_{\text {avg }}\left(r_{s}, t\right)=\frac{1}{K} \sum_{k=1}^{K} \mathbf{L}_{k}^{+}\left(r_{s} ; r_{h, k}\right) \cdot \mathbf{X}_{k}(t)
$$

Alternatively, information about the head positions during the recording is first averaged over repetitions and then represented in a spatially blurred version of the lead field:

$$
\mathbf{L}_{\text {avg }}=\frac{1}{K} \sum_{k=1}^{K} \mathbf{L}_{k}\left(r_{s} ; r_{h, k}\right)
$$

Inversion of this trial-averaged lead field, in combination with the trial-averaged data, leads to the forward calculation corrected estimate:

$$
\hat{\boldsymbol{s}}_{\text {avg }}\left(r_{s}, t\right)=\mathbf{L}_{\text {avg }}^{+} \cdot \frac{1}{K} \sum_{k=1}^{K} \mathbf{X}_{k}(t)
$$

The implementation according to Eq. (6) has been shown to be significantly less noise-sensitive than Eq. (4) (Uutela, et al., 2001) because the inverse operator is typically obtained using a regularization of the lead field $\mathbf{L}$ with an estimate of the noise covariance, which can be more robustly estimated across multiple trials compared to on a single trial level (see also (Dale \& Sereno, 1993)).

Note that when estimation of the source amplitude is followed by a forward modeling step towards a new set of sensor positions $r_{0}$, the realigned magnetic field distribution $\mathbf{X}_{0}$ as described above (Knosche, 2002; Numminen, et al., 1995) can be obtained:

$$
\mathbf{X}_{\mathbf{0}}(t)=\mathbf{L}\left(r_{s} ; r_{0}\right) \cdot \hat{\mathbf{s}}\left(r_{s}, t\right)
$$

where $\mathbf{X}_{0}$ can be either estimated per trial using $\hat{\mathbf{S}}_{\mathbf{k}}$ or for the average using $\hat{\mathbf{S}}_{\text {avg. }}$. The SSS-based MaxFilter method (Taulu, et al., 2005) is conceptually comparable to this, except that it uses lead fields based on a harmonic expansion of the magnetic field in the volume of the sensor array rather than lead fields based on a neurophysiologically inspired dipole source model.

The methods described above can only approximate (i.e. inter-/extrapolate) the data that would have been measured if there had been no head movements, to the level that the assumptions of those methods (e.g. field distributions and stationarity of the signal-to-noise) are met. Furthermore, these methods are all relatively complicated modeling approaches that need detailed additional information and choices to be made, such as geometric information with respect to the source and volume conduction model when using an inverse/ forward modeling approach, the selection of cut-off values in the regularization of the lead field inversion (Hamalainen \& Ilmoniemi, 1994), or the number of expansion coefficients when using spherical harmonic functions (Nurminen, Taulu, \& Okada, 2008; Taulu, et al., 2005). As a consequence, these methods are not guaranteed to work robustly in a wide variety of experimental situations.

Here we introduce and quantify the performance of head movement compensation techniques that are not based on sensor interpolation or lead field adjustments. Crucially, we demonstrate that the proposed tools can be 
readily applied to a wide range of tasks and experiments. First, we test a realtime head localizer that provides continuous visual feedback about the subject's current head position; both to the experimenter and to the subject (see section 2.5 and 2.7). This allows for accurate online repositioning of the head between separate recordings, reducing the between-sessions variance. Second, we use general linear modeling (GLM; see section 2.8) to remove head movement related trial-by-trial variance from the data (Worsley \& Friston, 1995), both at the sensor- and source level. By incorporating time-varying head position in the data analysis, the GLM allows to reduce the effect of movements.

We study the application of these two techniques in MEG experiments with various tasks covering different sensory modalities. We assess the consequences to the variance in the position of a dipole fitted to somatosensory evoked fields (Haegens, Osipova, Oostenveld, \& Jensen, 2010; Litvak et al., 2007; Medvedovsky, et al., 2007) and to the t-statistics of source-reconstructed ('beamformed') visually induced gamma-band activity (Hoogenboom, Schoffelen, Oostenveld, Parkes, \& Fries, 2006) and ('minimum-norm estimated') auditory evoked fields (Todorovic, van Ede, Maris, \& de Lange, 2011). For validation, we test the performance of the offline GLM method on data from three large MEG group studies.

\section{Methods}

The data for this study comprises two parts. For the first part we use MEG recordings to investigate the performance for single subject data. These recordings were performed specifically to test the online head repositioning method and as a proof of principle for the offline GLM method. For the second part we address the performance of the offline GLM method for group analysis, using MEG data from three previously performed group MEG studies.

\section{Subjects}

For the single subject analyses, two subjects, $M N$; male, aged 29, $L B$; female, aged 25 , took part in this study. They had no history of psychiatric or neurological problems and had normal $(L B)$ or corrected-to-normal $(M N)$ vision. Both subjects gave informed consent according to institutional guidelines of the local ethics committee (CMO Arnhem-Nijmegen, The Netherlands).

For the group analyses, we used MEG datasets obtained from studies performed by colleagues and that contained similar experimental stimuli as those used in the single subject recordings. The first dataset $(\mathrm{N}=16)$ originated from a group study on somatosensory spatial attention (Haegens, Luther, \& Jensen, 2012). The second dataset $(\mathrm{N}=32)$ was taken from a study investigating the genetic determination of visually induced gamma-band peak frequency (van Pelt, Boomsma, \& Fries, 2012). A study on neuronal suppression in auditory cortex (Todorovic \& de Lange, 2012) provided us with the third dataset $(\mathrm{N}=$ 20).

\section{Experimental design and procedures}

We here first describe the single subject experimental paradigm. To test whether repositioning of the subject's head using a real-time head localizer (see section 2.5) improves the consistency and statistical sensitivity of the data, we recorded and analyzed two pairs of measurements (see Figure 1), totaling four experimental recording sessions labeled A, B, C and D. Each session was started by positioning the subject in the MEG scanner. At the beginning of session $A$ the subject was placed comfortably in the scanner. At the beginning of session $\mathrm{B}$ the subject was repositioned to session A. At the beginning of session $\mathrm{C}$ the subject was not repositioned, but again placed comfortably in the scanner. At the beginning of session $\mathrm{D}$ the subject was repositioned to session $\mathrm{B}$. One measurement pair comprises data recorded in sessions B and D ('dataset BD'; with repositioning), another that of sessions A and C ('dataset AC'; without repositioning). We evaluated the consistencies in task-specific effects evoked during three tasks each involving a different sensory modality (see section 2.3) The specific tasks were selected for their wide coverage and relevance in recent cognitive research questions and serve to demonstrate the applicability of the investigated techniques to a wide range of tasks and experiments.

Subjects visited the laboratory on two separate days, taking part in four sessions in total (see Figure 1). Each session involved the three tasks explained below (see section 2.3). The first two sessions (sessions A and B) were recorded on day I, the other sessions (sessions C and D) were recorded at a similar time on day II (Figure 1). By recording the sessions on two separate days, we reduce the differences in evoked and induced responses due to fatigue and other timespecific effects. Subject $M N$ re-visited the lab two weeks after the first session and subject $L B$ re-visited the lab on the subsequent day. Within each session the subjects were instructed to maintain the same head posture during the three consecutive tasks. This instruction was identical to the normal procedures in our MEG lab. The subjects were not informed about the purpose of the study regarding the head movements, but were debriefed following the last session. To keep the subjects naive with respect to the reason of leaving the scanner prior to session B on day I and D on day II, they were instructed to perform a 
reading test outside the scanner in which they had to pronounce 50 English words as quickly as possible ( $\sim 1 \mathrm{~min}, M N$; 59 and $49 \mathrm{~s}, L B ; 64$ and $54 \mathrm{~s})$.

The group MEG studies each involved a specifically designed experimental paradigm addressing the respective cognitive research question. Detailed descriptions of the experimental paradigms can be found elsewhere (Haegens, et al., 2012; Todorovic \& de Lange, 2012; van Pelt, et al., 2012).

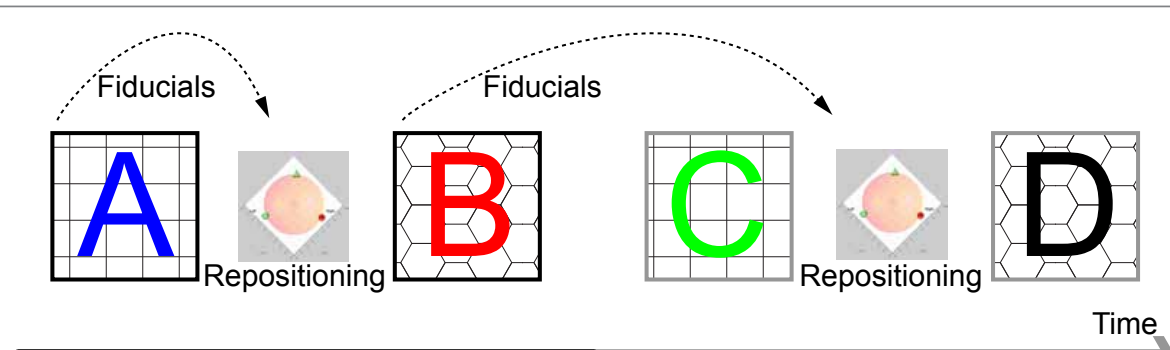

Day I

Day II

Figure 1. Experimental design. Subjects' head positions and task-induced brain activity were recorded during four sessions (A, B, C, and D) on two separate days. Prior to recording session B on day I, subjects repositioned their heads to their initial position of session A using a real-time head localizer that displayed the real-time fiducial locations in 3-D. On day II, repositioning was done prior to the second session of that day (session D). The target fiducial locations for session D were the initial locations obtained from session B. The analyses focused on the combinations of recordings during sessions B and D (with repositioning) and on those of sessions A and C (without).

\section{Materials}

A single MEG session ( $\sim 30 \mathrm{~min})$ of a single subject recording consisted of three consecutive tasks involving different sensory modalities. In each task, a small fixation cross was presented in the center of the projection screen during stimulus presentation and inter-stimulus intervals (ISI). Subjects were instructed to fixate on this cross to reduce ocular activity. Trials were interspersed with occasional blank periods when the subjects were encouraged to blink.

In the first task (tactile task, $\sim 10 \mathrm{~min}$ ), subjects' median nerves were stimulated by applying an electrical pulse to the left index finger (Haegens, et al., 2010;
Litvak, et al., 2007; Medvedovsky, et al., 2007). The stimuli were delivered with a constant current high voltage stimulator (Digitimer, Hertfordshire, UK). The intensity (350-600 mA, average $500 \mathrm{~mA}$ ) of the $200 \mu$ s electric pulses was set to a salient, yet comfortable level as individually established prior to the recordings. The task consisted of 200 trials lasting $\sim 2.5$ seconds each (ISI 2000-3000 ms, tactile stimulation $200 \mu$ s, blink period every 7 trials for 2500 $\mathrm{ms})$.

In the second task (visual task, $\sim 12 \mathrm{~min}$ ), subjects were visually stimulated with a foveal, circular sine wave grating (Hoogenboom, et al., 2006). The sine wave grating contracted inward, toward the fixation point (diameter $5 \mathrm{deg}$, spatial frequency 2 cycles/deg, contrast $100 \%$, velocity $1.6 \mathrm{deg} / \mathrm{s}$, duration 1350 ms). The task consisted of 200 trials lasting $\sim 3.5$ seconds each (ISI 1500-2500 ms, visual stimulation 1350 ms, blink period every 10 trials $2500 \mathrm{~ms}$ ).

In the third task (auditory task, $\sim 6 \mathrm{~min}$ ), brief tones (frequency $1000 \mathrm{~Hz}$, duration $5 \mathrm{~ms}, \sim 70 \mathrm{~dB}$ SPL) were presented binaurally via MEG-compatible air tubes (Todorovic, et al., 2011). The task consisted of 250 trials lasting $\sim 1$ second each (ISI 1000-1250 ms, auditory stimulation $5 \mathrm{~ms}$, blink period every 10 trials $2500 \mathrm{~ms}$ ).

All tasks contained deviant trials (omission of a pulse in the tactile task, outward contracting sine wave gratings in the visual task, and $1050 \mathrm{~Hz}$ tones in the auditory task). Subjects were instructed to count the deviants to encourage them to remain engaged throughout the experiment and attending the stimuli in all three tasks. A simple query at the end of each task ('How many deviants did you count?') was used for a check. Subjects had a binomial choice; e.g. 'More than 30' or 'Less than 30', to be answered with a single button press with the right index finger (average accuracy was 75\%). The stimuli were presented using a PC running Presentation software (Neurobehavioral Systems, Albany, CA, USA).

The stimuli used in the group MEG studies were comparable to those used in the single subject recordings described above; i.e. electrical pulses $(97 \pm 2.25$ trials per subject; mean \pm SD) delivered to the left thumb at $150 \%$ threshold level in the first study (Haegens, et al., 2012), a foveally presented circular sine wave grating that contracted toward the fixation point ( 2.7 cycles/deg; velocity, $0.75 \mathrm{deg} / \mathrm{s}$; contrast 100\%; $140 \pm 23$ trials) in the second study (van Pelt, et al., 2012), and presentations of brief tones ( $20 \mathrm{~ms} ; 1318 \mathrm{~Hz}$; $75 \mathrm{~dB}$ SPL; 150 trials) in the third study (Todorovic \& de Lange, 2012). 


\section{Data acquisition}

Ongoing brain activity was recorded using a whole-head magnetoencephalograph (MEG) with 275 axial gradiometers (VSM/CTF Systems, Port Coquitlam, British Columbia, Canada) in seated position (analog low-pass filter, $300 \mathrm{~Hz}$; sampling rate, $1200 \mathrm{~Hz}$ ). The subject's head position relative to the MEG sensors was measured before, during, and after each session using localization coils, placed at anatomical fiducials (nasion, left and right ear canals). High-resolution anatomical images of the whole brain for forward model generation were acquired (voxel size $=1 \mathrm{~mm}^{3}$ ) using a 1.5-T Siemens Avanto scanner (Erlangen, Germany). During MR acquisition, identical earplugs (now with a drop of Vitamin E in place of the MEG localization coils) were used for co-registration of the MRI and MEG data.

\section{Online head repositioning}

The CTF MEG system comes with three head localization coils, which are small coils that, when energized by an electrical current, result in a field distribution that approximates magnetic dipoles. Each coil is driven with sinusoidal current at a unique frequency and the combined field distribution is measured by the MEG channels. The contribution from each coil is extracted using spectral line extraction, and a magnetic dipole fit is performed on the extracted signals for each of the frequencies to determine the position of the corresponding coil. For each coil, the data contains the $\mathrm{x}-$, $\mathrm{y}$-, and z-coordinates in a 3-D cartesian coordinate system defined relative to the dewar.

The CTF MEG system also provides a shared memory interface in which the data is available for real-time analysis. Software from the open source FieldTrip toolbox (Oostenveld, Fries, Maris, \& Schoffelen, 2011) transfers this data from shared memory to a FieldTrip buffer on the acquisition computer. The data in this FieldTrip buffer is read on another computer over a TCP connection, which gives it access to the real-time coordinates of the head localization coils. The implementation we used in the current study is specific for the CTF MEG system, but the real-time software interface has recently been implemented for Neuromag MEG systems as well (Sudre et al., 2011).

The subjects reposition their heads with the aid of a visual projection using a projector and a screen mounted in front of the subject. A 3-D figure is displayed with a target head position. The figure shows the positions of the localization coils from a previous recording session, together with the real-time position of the localization coils. The subject's instruction is to match the real-time localization coil positions with the target positions, by moving his head until the graphical markers are overlaying. The distance to the target location is color-coded to aid the subject (Anim. S1). All online software is freely available as part of the FieldTrip toolbox ((Oostenveld, et al., 2011), see also http://www. ru.nl/donders/fieldtrip).

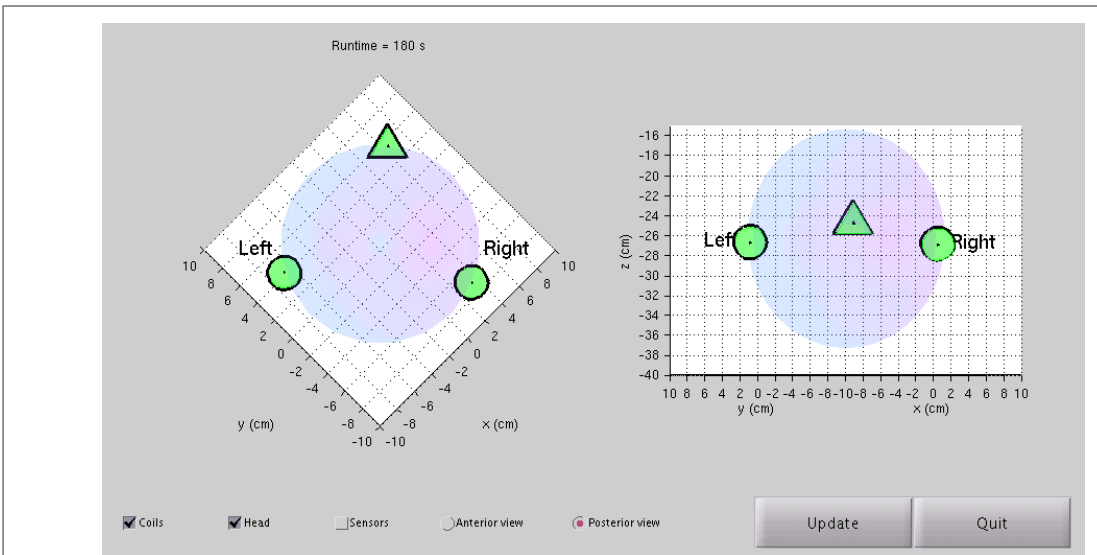

Anim. S1. Screenshot of the real-time head localizer, captured during the recording of session B of subject MN (this supplemental animation can be viewed in the online article version of this chapter). Repositioning of a subject can be performed by visualizing the anatomical fiducials from a reference dataset (nasion, left and right ear canals, black unfilled markers) and the graphically updating of the real-time head position. To aid the subject with repositioning, the real-time fiducial positions are color coded to indicate the distances to the targets (green $<1.5 \mathrm{~mm}$, orange $<$ $3 \mathrm{~mm}$, and red $>3 \mathrm{~mm}$ ).

\section{Data analysis}

Data were analyzed offline using the FieldTrip toolbox and MATLAB (MathWorks, Natick, MA, USA). Trials with muscle and SQUID artifacts were removed from the MEG time-series using standardized procedures.

For the single subject analyses, this resulted in $90-100 \%$ (mean $=97 \%$ ) of the original (non-deviant) trials being included for further analysis. The data was segmented around each stimulus presentation and baseline corrected using an interval of $100 \mathrm{~ms}$ before the occurrence of the stimulus. The analyses consisted of computing the somatosensory evoked fields (SEF; tactile task; 40-50 ms, (Haegens, et al., 2010; Litvak, et al., 2007; Medvedovsky, et al., 2007)), visually induced gamma-band activity (visual task; 0-500 ms, 65 $\pm 10 \mathrm{~Hz}$, (Hoogenboom, et al., 2006)) and auditory evoked fields (AEF; auditory task; 90-100 ms, (Todorovic, et al., 2011)) 
The general sensor level analysis of the three tasks was performed by testing the SEF peak, visually induced gamma-band activity, and AEF peak against the activity in prestimulus baseline using non-parametric cluster-based permutation paired samples t-tests (Maris \& Oostenveld, 2007). For the SEF and AEF the baseline was selected from -100 to $0 \mathrm{~ms}$. For the visually induced gamma-band activity the baseline was selected from -200 to $0 \mathrm{~ms}$. Only the last 150 trials of each task were used for the analyses to ensure that signal-to-noise ratios, degrees of freedom, and adaptation effects were matched between sessions.

The source modeling of the median nerve SEF implicated fitting equivalent current dipoles models to the data using a single-shell volume conduction model of the brain (Nolte, 2003). This volume conduction model was co-registered to each subject's sensor locations; we chose to use the average location of the combined sessions' head positions at recording onsets. The consistency of the position of the fitted dipole in a pair of measurements was determined through bootstrapping (1000 repetitions) of the concatenated trials (150 from one session, 150 from another). We chose to describe the variance in dipole position by quantifying the volume of the $95 \%$ confidence ellipsoid. This volume is defined by computing the multivariate (3-dimensional) eigenvectors and their values to estimate the $95 \%$ confidence according to McIntyre and colleagues (McIntyre, Stratta, \& Lacquaniti, 1998).

Sources of visually induced gamma-band activity were reconstructed using DICS, a frequency domain 'beamforming' approach (Gross et al., 2001; Van Veen, van Drongelen, Yuchtman, \& Suzuki, 1997). This method constructs a spatial filter for each of the 3-dimensional grid positions covering the brain (10 $\mathrm{mm}$ spacing), which passes activity from each grid location with unit gain, while maximally suppressing activity from all other sources. The beamformer spatial filter is constructed from the lead field and the cross-spectral density matrix of the data. The lead field is the physical forward model of the field distribution calculated from an assumed source at a given location and the subject-specific volume conduction model. The sessions were beamformed separately using session- and subject-specific lead fields and cross-spectral density matrices. The single-trial beamformer estimates of the two sessions were concatenated and their consistencies were evaluated by comparing the t-statistics (task vs. baseline activity) for dataset AC to that of dataset BD.

Sources of the AEFs were reconstructed using minimum-norm estimates. This method is a distributed inverse solution, constrained by a minimum-norm current estimate. The source space consisted of a large number of equivalent current dipoles placed on the cortical surface (preprocessed with the FreeSurfer and MNE Suite software packages, Martinos Center for Biomedical Imaging, Charlestown, MA, USA). It estimates the amplitude of all modeled source locations simultaneously and recovers a source distribution with minimum overal energy that produces data consistent with the measurement. This distributed source reconstruction was applied to each session before concatenation and subsequent statistical analyses (task vs. baseline activity).

With respect to the data obtained from the group MEG studies, we performed analyses similar to the general sensor level analyses described above. Separately for each study, single-subject statistical assessments were made and the peak activations (i.e. the absolute maximum of the subject t-statistics) were then tested against null at the group-level. The statistical assessments in the first study were made by comparing somatosensory evoked activity in the right hemisphere around 50-60 ms after stimulus onset (i.e. around the P60 trough), with activity in a $100 \mathrm{~ms}$ baseline period $(97 \pm 2.25$ trials, mean $\pm \mathrm{SD})$. For the second and third study respectively, comparisons were made between gammaband activity induced during a $600 \mathrm{~ms}$ task period and activity in $600 \mathrm{~ms}$ prestimulus baseline (140 \pm 23 trials), and between auditory evoked activity around $90-100 \mathrm{~ms}$ (i.e. around the N1 peak) and activity in $100 \mathrm{~ms}$ prestimulus baseline (150 trials).

\section{Testing the online head repositioning procedure}

The circumcenter of the three head localization coils, i.e. the center of the circle that passes through all the positions of the fiducials, was used as an index for head position. Differences in head positions between sessions were obtained by computing the position of the head on a trial-by-trial basis of each session. Subsequently, a principal component analysis was used to project these 3-D positions on the axes that explain the most variance. To test for systematic differences in head positions, we computed the statistical difference in head positions between the two pairs of sessions (between sessions A and C and between $\mathrm{B}$ and $\mathrm{D}$ ) using independent samples t-tests.

To test for changes in statistical sensitivity of the data due to online head repositioning (dataset $\mathrm{AC}$ vs. dataset $\mathrm{BD}$ ), we compare the two pairs of measurements on their task-specific statistical assessments (see section 2.6) at the source level. The sensor level statistics are affected by the proximity of the head to the sensors (Gaetz, et al., 2008). In the four sessions we did not explicitly control for this proximity, causing the measurement pairs AC and BD to have trivial differences in sensor level statistical values. 


\section{Testing the offline GLM-based head movement compensation}

The continuous representation of the fiducial locations is written to disk along with the MEG by the acquisition software. Trial-by-trial estimates of the position and the orientation of the circumcenter of the three fiducial markers were computed. These were demeaned and z-transformed to obtain the normalized deviants, i.e. translations $\left(H_{x}, H_{y}, H_{z}\right)$ and rotations $\left(H_{\varphi}, H_{\theta}, H_{\psi}\right)$, from the average head position and orientation. For both the sensor- and source level analyses, the data can be modeled with the following linear equation:

$$
\mathbf{Y}=\mathbf{b}_{\mathbf{0}} \mathbf{H}_{\mathbf{0}}+\mathbf{b}_{1} \mathbf{H}_{x}+\mathbf{b}_{2} \mathbf{H}_{y}+\mathbf{b}_{3} \mathbf{H}_{z}+\mathbf{b}_{4} \mathbf{H}_{\varphi}+\mathbf{b}_{5} \mathbf{H}_{\theta}+\mathbf{b}_{6} \mathbf{H}_{\psi}+\mathbf{E}
$$

where $\mathrm{Y}$ is a $1 \mathrm{xK}$ vector with the sensor- or source data over $\mathrm{K}$ trials, $\mathbf{b}_{0}$ is the intercept constant, $\mathbf{H}_{0}$ is a $1 \mathrm{xK}$ vector of ones, $\mathbf{b}_{1-6}$ are regression coefficients for the head position and orientation, and $\mathbf{E}$ is unexplained model error. The least squares solution to the linear equation,

$$
\min \|\mathbf{Y}-\mathbf{b H}\|^{2}
$$

results in a vector of $\mathbf{b}$ values per channel/voxel for each session of each subject. Subsequently, the estimated contributions of the regressors to the (source reconstructed) signal amplitude or spectral power can be removed from the original single-trial data:

$$
\mathbf{Y}_{\text {clean }}=\mathbf{Y}-\mathbf{b}_{1 . .6} \mathbf{H}_{x . \psi}
$$

where $\mathbf{Y}_{\text {clean }}$ represents the data with the movement related variance removed. Note that this general linear modeling (GLM) approach only affects the signal variance and not the signal mean over trials; i.e. the intercept constant $\mathbf{b}_{0}$ remains in the data.

An important aspect in the practical application is that the compensation should be performed in conjunction with the statistical analysis and cannot be done at an arbitrary point in the analysis pipeline. E.g., in the case of ERFs, the estimation of the regression coefficients $\mathbf{b}$ is performed separately for each channel and each latency, i.e. vector $\mathbf{Y}$ is formed for each channel and for each latency and the regression coefficients $\mathbf{b}$ are estimated for the specific channel and latency. In the case of powerspectra, the estimated regression coefficients are channel and frequency specific, and in the case of time-frequency responses they are channel, latency and frequency specific. Consequently, after compensation, the sensor level data cannot be used anymore for source modeling. To employ the GLM based compensation on the source level, single trial estimates for the cortical locations of interest have to be made from the original sensor level data preferably using a common spatial filter based on all trials. The beta weights are subsequently estimated for each cortical location and the variance in source amplitude over trials that is explained by the head movement is removed.

To account for the non-linear effects of head motion on the signal, we chose to consider also the squares, cubes and all their derivatives (the first three terms in the Taylor series expansion) of the head movement parameters in the model (resulting in a total of 36 regressors plus one constant). Following the removal of the trial-by-trial variance that was explained by the head movements, the task vs. baseline activation t-scores were computed and compared to the t-scores without the head movement compensation. We report descriptive statistics (t-score histograms and peak values) of the single subject analyses at the sensor and source level. Furthermore, we document how the GLM-based head movement compensation affects group level inferential statistics (second level effect sizes) by employing this technique on the single-subject data from the three group MEG studies.

\section{Results}

\section{Online head repositioning}

Subjects were consistently able to reposition their heads, effectively reducing intersession distances in head position at recording onsets; from 4.8 and 6.8 $\mathrm{mm}$ to 1.2 and $2.0 \mathrm{~mm}$ for subject $M N$ and $L B$ respectively (see Table 1). Visual inspection showed that the lack of repositioning resulted in session $\mathrm{C}$ to be an outlier (shown in green in Figure 2), while the head positions during sessions A, $\mathrm{B}$ and $\mathrm{D}$ were closer to each other. It can also be observed from Figure 2 that the subjects' heads, throughout the $\sim 30$ min. recordings, slowly but progressively drifted away.

Repositioning improved the consistencies of head positions in paired datasets as indicated by smaller statistical intersession difference in the positions; for subject $M N, \mathrm{t}(1077)=-74$ with, and $\mathrm{t}(1100)=-100$ without repositioning; for subject $L B, \mathrm{t}(1155)=19$ with, and $\mathrm{t}(1151)=351$ without repositioning This improvement was most pronounced early after repositioning, i.e. during the tactile task; subject $M N, \mathrm{t}(307)=-57$ with, and $\mathrm{t}(330)=-180$ without repositioning; subject $L B, \mathrm{t}(332)=15$ with, and $\mathrm{t}(343)=314$ without repositioning 


\begin{tabular}{|c|c|c|c|}
\hline \multirow[t]{2}{*}{ Sessions } & \multirow[t]{2}{*}{ Time } & \multicolumn{2}{|c|}{ Distance (mm, t-value*) } \\
\hline & & Subject $M N$ & Subject $L B$ \\
\hline \multirow{5}{*}{$\begin{array}{l}\text { A and C (without } \\
\text { repositioning, } \\
\text { different day) }\end{array}$} & Recording onset & 4.8 & 6.8 \\
\hline & Tactile task (0-10 $\mathrm{min})$ & $5.4, \mathrm{t}(330)=-180$ & $7.1, t(343)=314$ \\
\hline & Visual task (11-22 min) & $4.5, \mathrm{t}(341)=-84$ & $7.1, \mathrm{t}(358)=267$ \\
\hline & Auditory task (23-28 min) & $4.1, \mathrm{t}(425)=-196$ & $8.4, \mathrm{t}(446)=342$ \\
\hline & Throughout recording & 4.6, $\mathrm{t}(1100)=-100$ & $7.5, \mathrm{t}(1151)=351$ \\
\hline \multirow{5}{*}{$\begin{array}{l}\text { B and D } \\
\text { (with } \\
\text { repositioning, } \\
\text { different day) }\end{array}$} & Recording onset & 1.2 & 2.0 \\
\hline & Tactile task (0-10 min) & $2.7, \mathrm{t}(307)=-57$ & $1.3, \mathrm{t}(332)=15$ \\
\hline & Visual task (11-22 min) & $4.3, \mathrm{t}(332)=-109$ & $2.0, \mathrm{t}(357)=4$ \\
\hline & Auditory task (23-28 min) & $6.2, \mathrm{t}(434)=-215$ & $4.9, \mathrm{t}(462)=103$ \\
\hline & Throughout recording & $4.6, \mathrm{t}(1077)=-74$ & $2.9, \mathrm{t}(1155)=19$ \\
\hline \multirow{5}{*}{$\begin{array}{l}\text { A and } B^{* *} \\
\text { (with } \\
\text { repositioning, } \\
\text { same day) }\end{array}$} & Recording onset & 2.0 & 2.7 \\
\hline & Tactile task (0-10 $\mathrm{min})$ & $2.3, \mathrm{t}(317)=34$ & $1.2, \mathrm{t}(338)=10$ \\
\hline & Visual task (11-22 $\mathrm{min})$ & $3.8, \mathrm{t}(339)=33$ & $2.2, \mathrm{t}(363)=-5$ \\
\hline & Auditory task (23-28 $\mathrm{min})$ & $6.3, t(425)=214$ & $3.9, \mathrm{t}(458)=-82$ \\
\hline & Throughout recording & $4.3, t(1085)=48$ & $2.2, \mathrm{t}(1163)=-14$ \\
\hline
\end{tabular}

\begin{tabular}{|c|c|c|c|c|c|c|c|c|c|}
\hline \multirow[t]{2}{*}{ Task } & \multirow[t]{2}{*}{ Subject } & \multicolumn{4}{|c|}{ Without repositioning } & \multicolumn{4}{|c|}{ With repositioning } \\
\hline & & \multicolumn{2}{|c|}{ standard } & \multicolumn{2}{|c|}{ GLM } & \multicolumn{2}{|c|}{ standard } & \multicolumn{2}{|c|}{ GLM } \\
\hline \multirow[t]{2}{*}{ Tactile } & $M N$ & $0.88 \mathrm{~cm}^{3}$ & t 12.6 & n.a. & $\mathrm{t} 13.2$ & $0.58 \mathrm{~cm}^{3}$ & $\mathrm{t} 10.8$ & n.a. & $\mathrm{t} 11.5$ \\
\hline & $L B$ & $0.18 \mathrm{~cm}^{3}$ & $\mathrm{t} 20.8$ & n.a. & $\mathrm{t} 23.1$ & $0.14 \mathrm{~cm}^{3}$ & $\mathrm{t} 24.5$ & n.a. & $\mathrm{t} 27.0$ \\
\hline \multirow[t]{2}{*}{ Visual } & $M N$ & $\mathrm{t} 11.9$ & $\mathrm{t} 9.8$ & $\mathrm{t} 12.6$ & $\mathrm{t} 10.4$ & $\mathrm{t} 14.0$ & t 9.5 & $\mathrm{t} 14.9$ & t 10.1 \\
\hline & $L B$ & $\mathrm{t} 21.2$ & $\mathrm{t} 18.7$ & $\mathrm{t} 24.3$ & $\mathrm{t} 21.9$ & $\mathrm{t} 23.7$ & $\mathrm{t} 23.7$ & $\mathrm{t} 26.1$ & $\mathrm{t} 25.7$ \\
\hline \multirow[t]{2}{*}{ Auditory } & $M N$ & $\mathrm{t} 17.3$ & $\mathrm{t} 24.6$ & t 18.9 & $\mathrm{t} 26.3$ & $\mathrm{t} 17.2$ & $\mathrm{t} 25.0$ & t 18.0 & $\mathrm{t} 26.8$ \\
\hline & $L B$ & $\mathrm{t} 20.8$ & $\mathrm{t} 33.2$ & $\mathrm{t} 22.3$ & $\mathrm{t} 37.8$ & $\mathrm{t} 20.5$ & $\mathrm{t} 30.8$ & $\mathrm{t} 22.2$ & t 32.9 \\
\hline
\end{tabular}

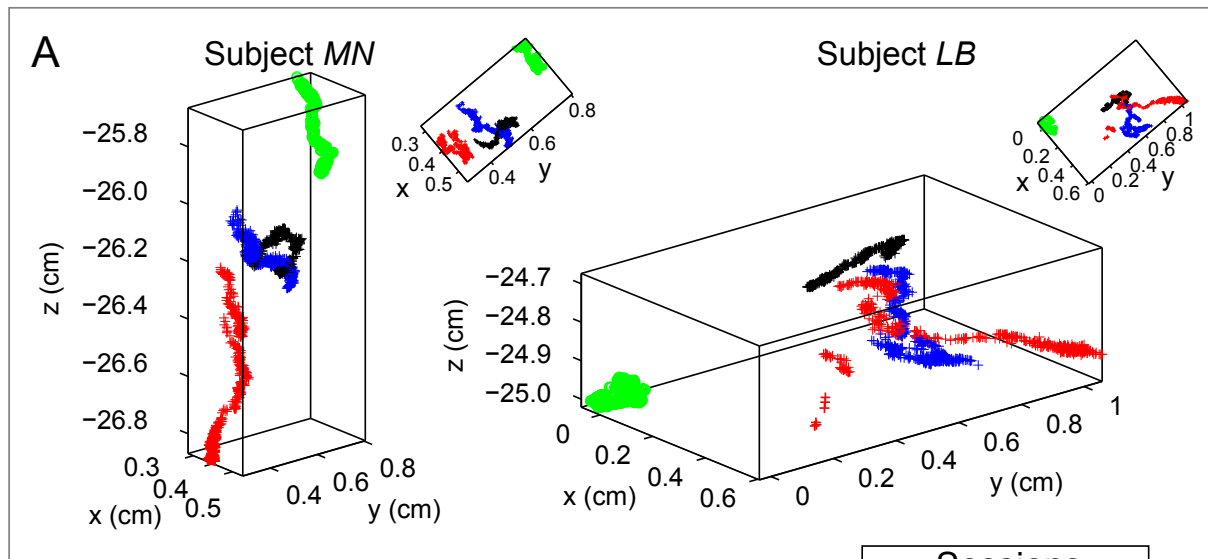

Head positions in MEG dewar coordinates

$+A \stackrel{\text { Sessions }}{+}+\mathrm{B} \circ \mathrm{C}+\mathrm{D}$
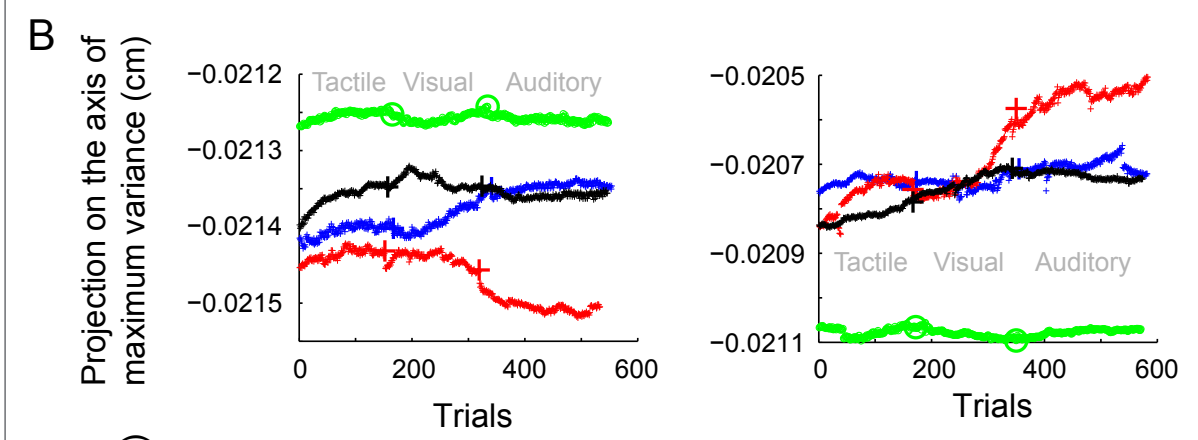

$\mathrm{C} \div \widetilde{\mathrm{E}}$
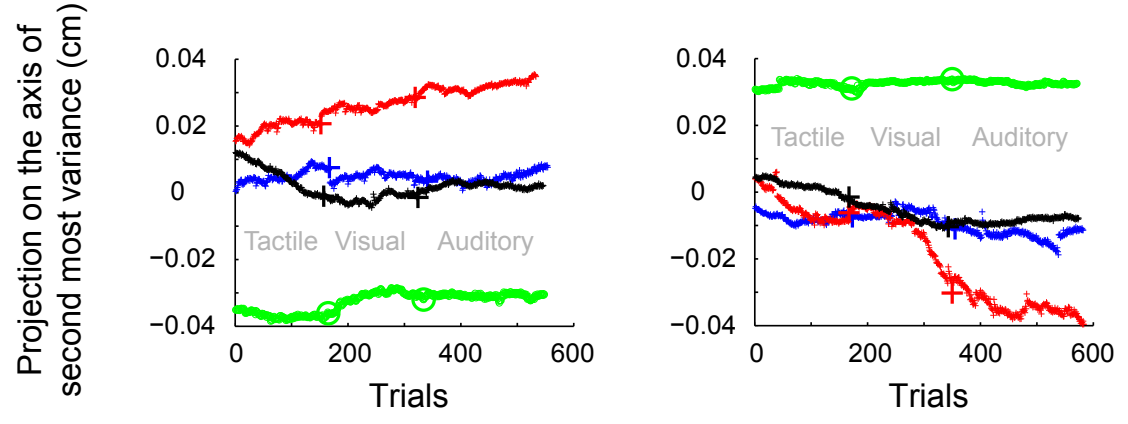

Figure 2. Head positions, as indexed by the circumcentra of the three head localization coils, throughout the recording sessions of subjects MN (left panel) and LB (right panel). (A) Trial-to-trial head positions plotted in MEG dewar coordinates. (B) Head positions plotted along the axis explaining most variance in the head positions of all four sessions together against trials, where zero is recording onset and the respective tasks are denoted. (C) Same as in B, but plotted along the axis of second most variance. 
We next investigated the consequences of repositioning on the source reconstructed data. For the median nerve somatosensory evoked fields (SEFs), repositioning of each subject reduced the variability in dipole fit positions (quantified by the 95\% confidence ellipsoid volume) from 0.88 to $0.58 \mathrm{~cm} 3$ and from 0.18 to $0.14 \mathrm{~cm} 3$ respectively for both subjects $M N$ and $L B$ (see Figure $3 \mathrm{~A} ;-28 \pm 8 \%$, mean $\pm \mathrm{SD}$ ). Furthermore, it enhanced peak t-statistics of visually induced gamma-band activity from 11.9 to 14.0 and from 21.2 to 23.7 (see Figure 3B; $15 \pm 4 \%$ ), but not that of auditory evoked fields (AEFs) in the chronologically last task; from 17.3 to 17.2 and from 20.8 to 20.5 (see Figure $3 \mathrm{C} ;-1 \pm 1 \%)$.

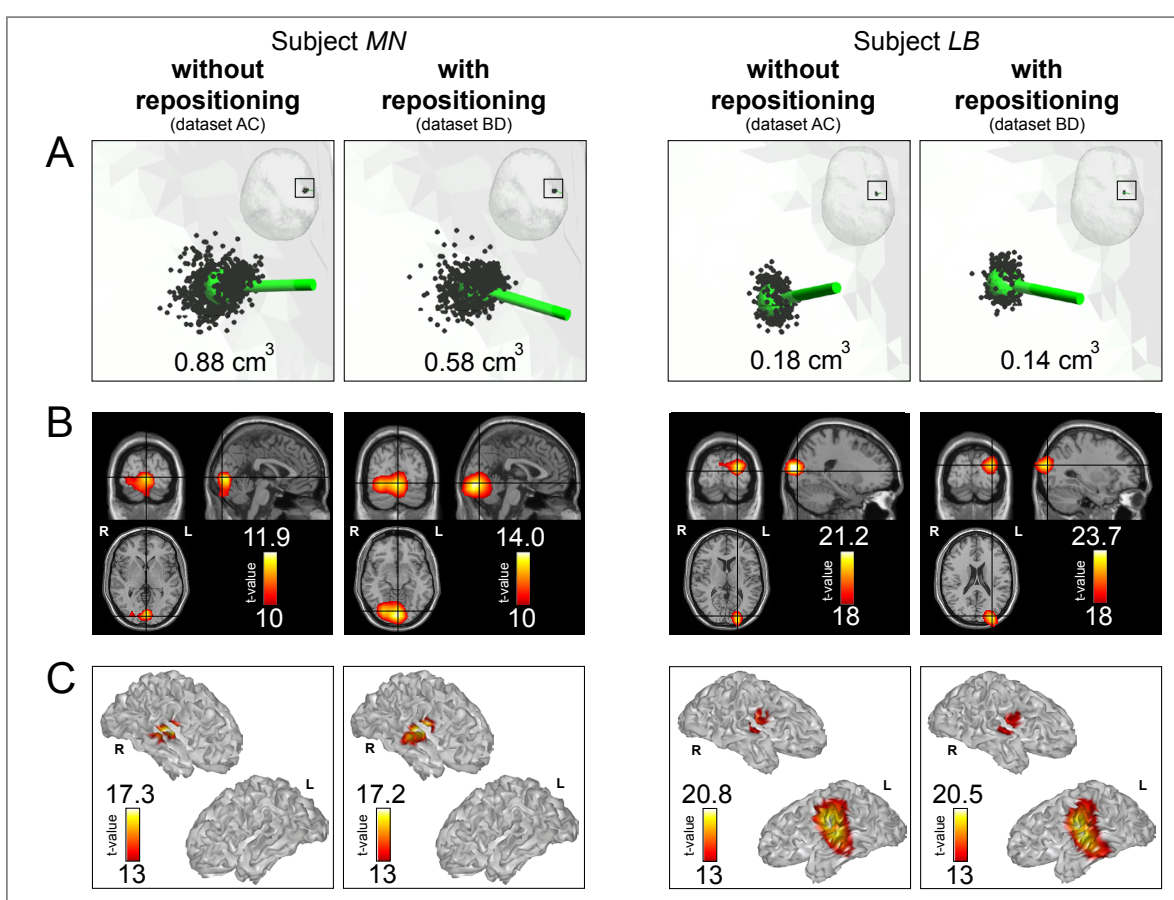

Figure 3. Source level consequences of online head repositioning between sessions for subjects $M N$ and $L B$. Panels follow the order of the experimental tasks. (A) Trials were randomly selected from concatenated SEF sessions, averaged and dipole fitted (1000 iterations). The variance in dipole position (black dots) of median nerve somatosensory evoked fields $(40-50 \mathrm{~ms})$ was described by quantifying the volume of the $95 \%$ confidence ellipsoid. The green marker represents the averaged dipole position and its orientation. (B) Source localization (beamforming) of visuallyinduced gamma-band activity $(65 \pm 10 \mathrm{~Hz}, 0-500 \mathrm{~ms})$. The threshold of the color axis was raised in order to resolve the spatial structure around the statistically significant peaks. The upper t-values are the peaks. (C) Source localization (minimum-norm estimation) of auditory evoked fields $(90-100 \mathrm{~ms})$.

\section{Offline general linear modeling}

Head position and orientation confounds were estimated and their contribution was removed from the single-trial sensor- and source level data. Comparing peak t-statistics with and without this operation, we found GLM to yield improvements in all task vs. baseline activity contrasts of both subjects $M N$ and $L B$, both for sensor- and source level (see Table 2, $9 \pm 3 \%$ increase in peak t-statistics, overall mean $\pm \mathrm{SD}$ ). Example topographic plots and histograms show that, with regression analysis, the distribution of t-statistics overall appears increased with the shape preserved (Figure 4).

Employing the GLM-based head movement compensation on sensor level data from three existing group studies revealed t-statistics that were greater than without using this technique. On average, peak t-statistics of activity evoked during 97 electrical pulses delivered to the left thumb improved by $23 \% \pm$ $5 \%$ in the first study (mean \pm SEM, 16 subjects mean; from $\mathrm{t}(96)=1.09$ to $\mathrm{t}(96)=1.39, p<<0.001)$. In a similar vein, the effect size of the increase in peak t-statistics of induced gamma-band activity during 140 presentations of a circular sine wave grating in the second study was $29 \pm 4 \%$ (32 subjects mean; from $\mathrm{t}(139)=13.4$ to $\mathrm{t}(139)=16.9, p<<0.001)$. In the third study, head movement compensation yielded a $15 \pm 1 \%$ increase in absolute peak t-statistics of activity evoked during 150 presentations of brief tones (20 subjects mean; from $\mathrm{t}(149)=3.61$ to $\mathrm{t}(149)=4.15, p<<0.001)$.

\section{Discussion}

The present study investigated online and offline tools to compensate MEG data for head movement, as assessed through a statistical evaluation that is similar to how analysis is performed in most cognitive research projects. We recorded single subjects' brain activity in four sessions (Figure 1) during the performance of three tasks (tactile, visual, auditory) to demonstrate the applicability of the investigated techniques to a wide range of tasks and experiments. We analyzed data from three larger MEG group studies to further validate the effect of the offline GLM-based technique on statistical sensitivity.

Using a real-time head localizer, subjects were consistently able to accurately reposition their heads between sessions. We observed that online head repositioning effectively reduced inter-session differences in head position (Figure 2 and Table 1), thereby improving the statistical assessment of brain activity recorded during those sessions (Figure 3). Interestingly, we observed this improvement also when we analyzed the recordings separately and 
A

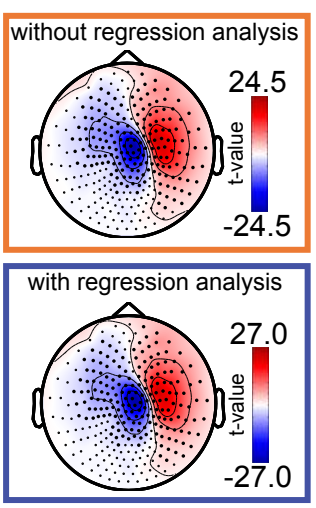

C

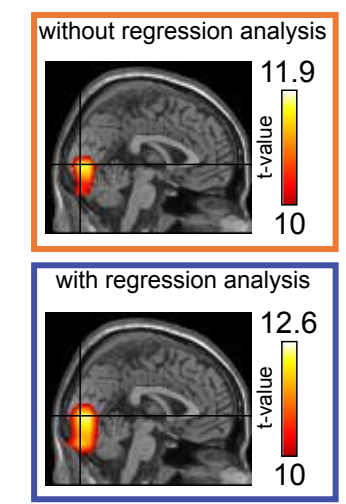

E

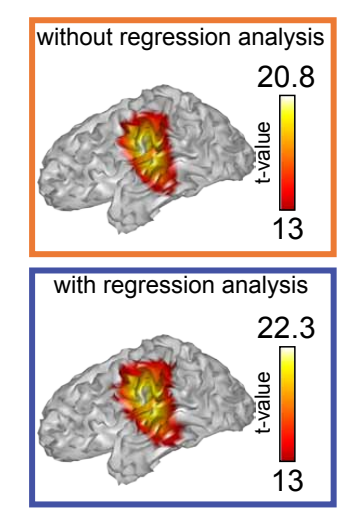

B

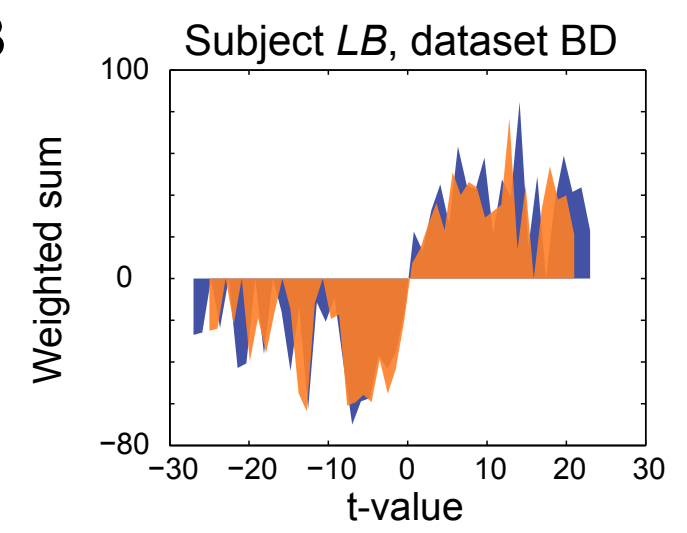

$\mathrm{D}$

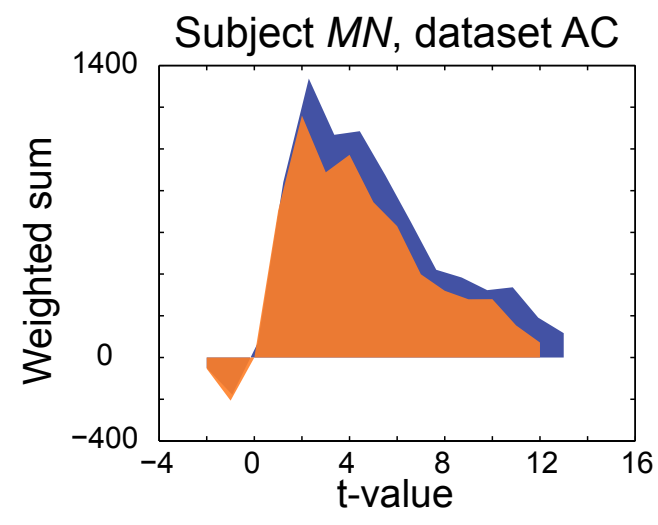

$F$

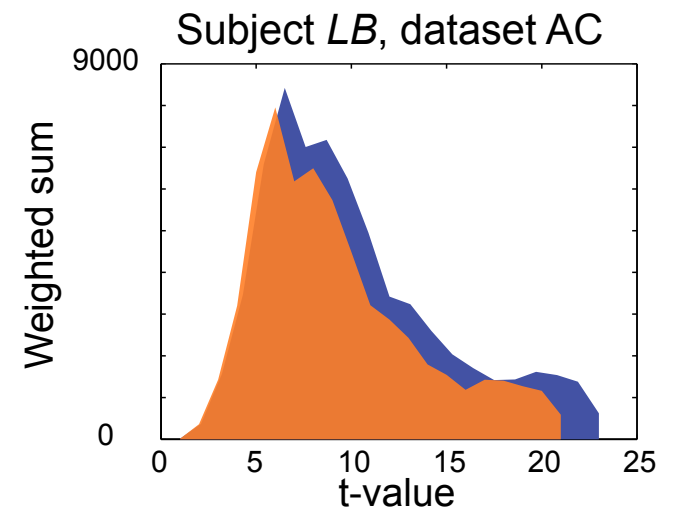

combined them in source space (Figure 3B). This finding suggests that in the combined analysis of two sessions, different head positions negatively affect the outcomes, even if accounted for by the source estimation. We hypothesize this to be due to differences in signal-to-noise ratio that are caused by differences in the distance between the sources and sensors, and due to the non-linear fitting of those sources.

Regression analyses, i.e. the offline incorporation of the head position timeseries into a general linear model (GLM), successfully reduced the confounding variance that was due to within-sessions head movements. Our subjects did not make abrupt movements but slowly drifted down (see Figure 2A and B), a movement that can be well compensated with offline general linear modeling (Figure 4). The use of GLM to remove confounding head motion is common practice in the analysis of functional magnetic resonance imaging (Worsley \& Friston, 1995). To our knowledge the present study is the first demonstration that it can successfully be applied to MEG recordings. The application of the GLM-based approach to a 16 subject study addressing somatosensory spatial attention (Haegens, et al., 2012) shows an improvement of statistical sensitivity, as assessed by an increase in t-scores, by $23 \%$. The application of the same method to a 32 subject visual attention (van Pelt, et al., 2012) and a 20 subject auditory expectation study (Todorovic \& de Lange, 2012) demonstrated an increase in t-scores of $29 \%$ and $15 \%$ respectively. In each study the initial statistical sensitivity was already sufficient to address the research question for the respective studies and the improvement with the GLM method did not change the inferences that were drawn from the data. However, the large increase in statistical sensitivity suggests that the GLM method allows future studies to be performed with smaller subject group sizes, or alternatively allows studies to be performed with smaller effect sizes. Furthermore, by compensating

Figure 4. Example statistical distributions with (blue) and without (orange) offline GLM-based head movement compensation. Each row displays one of the experimental tasks for one of the subjects. Note the different color scaling limits. (A) SEFs of subject LB, sessions B and D combined. Highlighted sensors indicate statistically significant differences ( $\mathrm{p}<0.05$, corrected for multiple comparisons). (B) Histogram of the t-values of all channels and timepoints in the 40-50 ms interval, with (blue) and without (orange) regression analysis. Each histogram bin is weighted by its corresponding t-value. (C) Visually-induced gamma-band activity $(65 \pm 10 \mathrm{~Hz}$, $0-500 \mathrm{~ms}$ ) of subject MN, sessions A and C combined. (D) Histogram of the t-values of all voxels. (E) AEFs (90-100 ms) of subject LB, sessions A and C combined. (F) Histogram of the t-values of all cortical mesh surfaces. 
for head movement related variance, this technique opens the way for cleaner investigations of trial-by-trial coupling of behavioral measures and the MEG signal.

The combination of both these online and offline tools yielded the largest improvement, i.e. online repositioning by means of a real-time head localizer reducing the between-sessions variance and offline GLM regressing out the within-sessions variance. The present study does not address the use of the real-time head localizer to reduce the variance within a session. We suggest that the real-time head localizer can also be used to compensate for withinsessions variance by correcting for head movement using a short repositioning instruction between experimental blocks. This could counteract the slow but progressive drifting away from the position at recording onset of the subject's head without the need for head fixation. Such an approach may be specifically of relevance for studies that include subject groups that find it more difficult to maintain a constant position over a long period of time.

Finally, we have demonstrated that the tools introduced here can be readily applied to a wide range of tasks and experiments. Compared to the existing offline compensation methods which are based on sensor interpolation or lead field adjustments (see Introduction of this chapter) and for which an implementation is not available for all MEG systems, the application of these tools is relatively straightforward and is made available in the open-source FieldTrip toolbox, which allows it to be employed on data from all commonly used MEG systems. The user does not need detailed additional information (e.g. source and head model) and does not need to make modeling specific choices. It remains to be seen how the tools presented here complement and compare to the existing methods when dealing with the consequences of head movements on statistical sensitivity. For instance, the within-session head repositioning, as suggested in this paper, may have a positive effect on the SSS-based sensor level interpolation method (Taulu, et al., 2005) by virtue of reducing trial-bytrial variations of head position and inherent improvements in signal-to-noise ratio. In a similar vein, it would be interesting to study how general linear modeling enhances statistical sensitivity after incorporation of head position information into the source reconstruction procedure (Uutela, et al., 2001). The latter method, either by the relatively noise-sensitive adjustment of the lead field matrix on a trial-by-trial basis (see Eq. (4)) or using an averaged, spatially blurred version of the lead field (see Eq. (5) and (6)), has proven to reduce source localization error after head movement (Uutela, et al., 2001). However, to date it has not been investigated whether and how it affects statistical sensitivity. The blurred version provides a static solution for the inverse source estimation and therefore cannot account for the dynamic trial-by-trial variation in the relative location of the sources (see Appendix A for an initial exploration of this method on the present data). Consequently, it does not contribute to the statistical power, which we aimed at with the present study.

In summary, we consider the real-time head localizer tool a valuable addition to the experimental setup and the use of the GLM-based head movement compensation a necessary attribute of the MEG analysis toolbox. 


\section{Appendix A}

Forward calculation correction and statistical sensitivity

Forward calculation correction is a technique that incorporates the variability in head position into the forward calculations of the magnetic field (see Eq. (5) and (6)). For a dipole with an arbitrary location and orientation, the magnetic field picked up by a sensor coil at any position relative to the dipole can be computed. Variability in the exact location of the dipole relative to the sensor due to changes in head position can be taken into account by a computing a linear combination of the modeled magnetic fields for different sensor locations. We took a similar approach as proposed by Uutela and colleagues (Uutela, et al., 2001). For computational efficiency, rather than taking a plain average of the lead fields computed for each trial (and head position) separately, we computed a weighted average of a well-chosen set of lead fields. These sets of lead fields reflected a representative set of positions of the head relative to the sensors. We used k-means clustering on the single trial head position estimates and obtained 10 clusters. The centroid of each of these clusters was used as a head position for which the lead fields of the sources were computed. The numbers of trials contributing to each of the clusters were used as a weighting factor, with which the lead fields were weighted before they were summed across the clusters. The lead fields obtained using this approach were used for the source reconstruction of activity evoked in all three tasks (see section 2.6) and the statistical sensitivity was compared to that without using this correction (listed under 'Standard' in Table 2). The incorporation of variable head positions into the magnetic field forward calculations did not reduce the variance in dipole position of the SEFs, from 0.88 to $0.84 \mathrm{~cm} 3$ ('dataset AC') and from 0.58 to 0.60 $\mathrm{cm} 3$ ('dataset BD') for subject MN and from 0.18 to $0.22 \mathrm{~cm} 3$ and from 0.14 to $0.11 \mathrm{~cm} 3$ for subject LB ( $0 \pm 18 \%$, overall mean \pm SD). Furthermore, it did not significantly improve the peak t-statistics of beamformed visually induced gamma-band activity $(1 \pm 1 \%$, overall mean $\pm \mathrm{SD}$; subject $\mathrm{MN}$ : from $\mathrm{t}=11.9$ to 11.9 and from $t=14.0$ to 14.0 ; subject $L B$ : from $t=21.2$ to 21.4 and from $\mathrm{t}=23.7$ to 23.9$)$ and minimum-norm estimated AEFs $(1 \pm 3 \%$, overall mean $\pm \mathrm{SD}$; subject $\mathrm{MN}$ : from $\mathrm{t}=17.3$ to 17.4 and from $\mathrm{t}=17.2$ to 17.5 ; subject LB: from $t=20.8$ to 20.1 and from $t=20.5$ to 21.1).The lack of this method contributing to the trial-by-trial consistency, and thus the statistical sensitivity of the data, can be explained: the varying head position during the recording gets represented in a spatially blurred version of the actually measured magnetic field and the lead fields used in the forward calculation. Although the blurred lead field can provide a more accurate spatial topography of the true sources and thereby improve the accuracy of the reconstructed source location, the static lead field solution cannot account for the dynamic trial-by-trial variability that is represented as across-trial variance of the source positions and source amplitudes. 

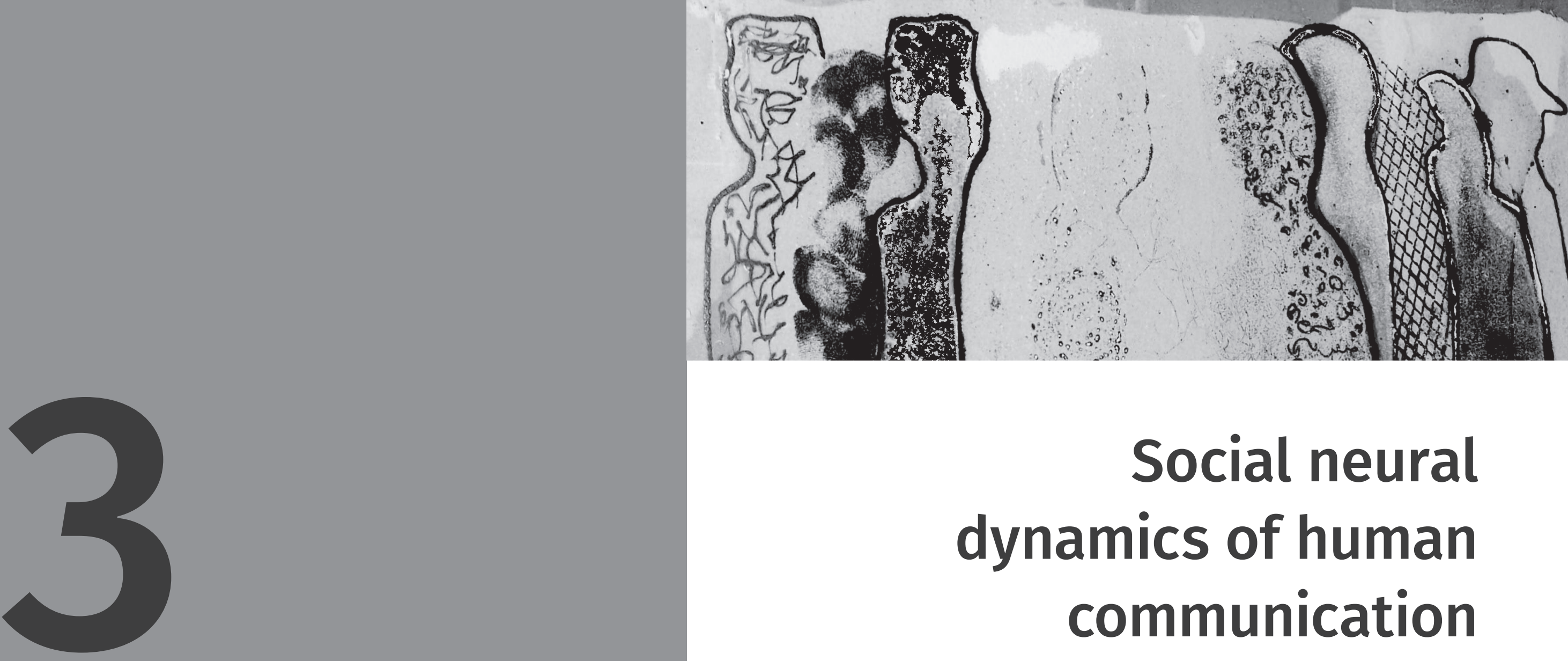

\author{
Social neural \\ dynamics of human \\ communication
}




\section{Neural mechanisms of communicative innovation}

Stolk A, Verhagen L, Schoffelen JM, Oostenveld

R, Blokpoel M, Hagoort P, van Rooij I, Toni I 


\section{Introduction}

We can modify reality by selecting either instrumental actions that change the physical state of the environment according to the mechanics of the action, or communicative actions that change the mental state of other agents according to the content of the action (Noordzij, et al., 2010; Searle, 2010). For instance, we can fill a glass with a drink, or ask a bartender to do that. A common language might help to achieve the latter by providing access to previously established shared symbols, but those symbols presuppose a computational mechanism powerful enough to negotiate them across interlocutors (Levinson, 2006). Here we study the electrophysiological correlates supporting the rapid negotiation of shared symbols, a fundamental property of human communication (Evans \& Levinson, 2009; Tomasello, 2008).

Given the vast number of possible meanings that can be attributed to a novel communicative action (Galantucci, 2005; Jablonka, 2002), it remains unclear how novel shared symbols can be rapidly selected and understood. Generalpurpose learning algorithms like temporal difference or Hebbian learning (Behrens, et al., 2009; Keysers \& Perrett, 2004) do not seem suitable, since they require many trials to converge on statistically relevant features. There are brain circuits that support fast predictions on sensory inputs or consequences of planned actions (Giese \& Poggio, 2003; Peelen, et al., 2009), but those circuits are geared towards a specific domain of application with well-defined priors (e.g. faces, (Adolphs, 2009)). Novel shared symbols, being novel, do not have welldefined priors (Fodor, 2000; Levinson, 2006; Sperber \& Wilson, 2001). Solving this type of communicative problems requires a mechanism that supports a rapid exploration through a large search space, generating connections between different conceptual structures (Gentner, 2003; Goldstone \& Rogosky, 2002).

a symbls, communication is often thought as coding-decoding a computational mechanism powerful enough to mutually negotiate them. Sharing the meaning of a novel symbol might rely on similar conceptual inferences across communicators, or on statistical similarities in their sensorimotor behaviors. Using magnetoencephalography, we assess spectral, temporal, and spatial characteristics of neural activity evoked when people generate and understand novel shared symbols during live communicative interactions. Solving those communicative problems induced comparable changes in the spectral profile of neural activity of both communicators and addressees. This shared neuronal upregulation was spatially localized to the right temporal lobe and the ventromedial prefrontal cortex, and emerged already before the occurrence of a specific communicative problem. Communicative innovation relies on neuronal computations that are shared across generating and understanding novel shared symbols, operating over temporal scales independent from transient sensorimotor behavior.

These theoretical considerations about human communication lead to three predictions on its underlying mechanism. First, given that establishing shared symbols requires taking into account the inferred knowledge of the interlocutor ("audience design", (Clark, 1996; de Ruiter, et al., 2010; Galantucci \& Garrod, 2011)), the generation and comprehension of those symbols should involve neural patterns associated with flexible conceptual knowledge (Derix, Iljina, Schulze-Bonhage, Aertsen, \& Ball, 2012; Kumaran, Summerfield, Hassabis, \& Maguire, 2009; Lambon Ralph, Sage, Jones, \& Mayberry, 2010; Siegal \& Varley, 2002), rather than sensorimotor couplings with limited generalization patterns (Hasson, et al., 2012; Jiang et al., 2012; Keysers \& Perrett, 2004; Orban de Xivry et al., 2011; Pickering \& Garrod, 2004). Second, cerebral activities supporting these conceptual processes during generation and comprehension 
of novel shared symbols should overlap, given that these processes relate to the specific conversational context shared by the interlocutors of the communicative exchange (Menenti, Pickering, \& Garrod, 2012). Third, cerebral activity supporting this predicted overlap should predate in time the processing of the communicative stimuli themselves, given that the meaning of any stimulus arises from a conceptual space defined by the ongoing communicative interaction (Clark, 1996; Stephens, Silbert, \& Hasson, 2010), rather than by the sensory material itself.

We test these predictions by characterizing spatial, spectral, and temporal features of neural activity supporting the planning and understanding of novel communicative actions, using an absolute index of source-reconstructed magnetoencephalographic activity (MEG). In contrast to previous work largely focused on individuals perceiving instrumental actions (de Lange, Spronk, Willems, Toni, \& Bekkering, 2008; Iacoboni et al., 2005) or known linguistic material (Lerner, Honey, Silbert, \& Hasson, 2011; Stephens, et al., 2010), here we investigated both production and comprehension of novel communicative actions during a live interaction between pairs of participants, and directly contrast those phenomena with a control interaction involving no communicative necessities (Figure 1).

\section{Materials and Methods}

\section{Participants}

Fifty-two participants (22 males, 30 females; ages 18-40), were recruited to take part in this study. They were screened for a history of psychiatric and neurological problems and had normal or corrected-to-normal vision. Participants gave informed consent according to institutional guidelines of the local ethics committee (CMO region Arnhem-Nijmegen, The Netherlands) and were either offered a financial payment or given credits towards completing a course requirement. MEG was acquired from one member of each pair. Two pairs of participants were excluded from data analysis due to MEG-system failure and muscle artifacts, leaving 24 pairs of participants for data analysis

Tasks

The communicative and the instrumental tasks are described in detail in Appendix A of this chapter.

\section{MEG and MRI data acquisition}

Brain activity was recorded over two sessions using a whole-head magnetoencephalograph (MEG) with 275 axial gradiometers (CTF275, VSM MedTech; $1200 \mathrm{~Hz}$ sampling rate, $300 \mathrm{~Hz}$ analog low-pass filter). Before the second session, each participant repositioned his/her head in the same location and orientation as the position measured before the first session, using a real-time head localizer tool (Stolk, Todorovic, Schoffelen, \& Oostenveld, 2013). Anatomical images of the brain for forward model generation (voxe size $=1 \mathrm{~mm}^{3}$ ) were acquired using a 1.5T Siemens Avanto scanner. During MR acquisition, identical earplugs (with a vitamin E capsule in place of the MEG localization coils) were used for co-registration of the MRI and MEG data.

\section{MEG data analysis}

Data were analyzed offline using the FieldTrip toolbox (Oostenveld, et al., 2011) and custom MATLAB code (MathWorks, Natick, MA, USA). Trials with muscle and SQUID artifacts were removed from the MEG timeseries, resulting in 91 $\pm 5 \%$ of the original trials being included for further analysis. Following our experimental rationale, we focused the analysis of the MEG data on the trial epochs during which the Communicator and Salesman planned their actions (epoch D: planning, Figure 1), and the Addressee and Roadworker observed the other player's movements (epoch E: observation). For each epoch, we also considered the preceding baseline period $(1 \mathrm{sec})$, during which only the empty grid was visible. We analyzed these task epochs in two ways, differing in the time scale at which the inferences can be drawn.

In Analysis \#1, we considered the whole time interval covered by the planning and observation events. Accordingly, we extracted the overall changes in cerebral neural activity evoked during those events, using adaptive spatial filtering ('beamforming', Appendix A of this chapter) to estimate local neural population activity throughout the brain as a function of frequency. We matched the signal-to-noise ratios of the different conditions within each participant by ensuring that each condition contributed the same number of samples to the data analysis. To achieve this, each trial was segmented into multiple consecutive non-overlapping windows of $500 \mathrm{~ms}$. For each participant, windows were randomly selected and excluded from subsequent analyses until the different conditions provided the same number of windows. Then the windowed time series from each trial epoch were tapered with a set of 4 orthogonal Slepian tapers prior to spectral estimation and the resulting estimates of the (cross-) spectral densities were averaged across tapers. This resulted in a spectral smoothing of $\pm 5 \mathrm{~Hz}$. 
In Analysis \#2, we extracted the fine-grained temporal dynamics of power changes during the task epochs mentioned above, performing a time-frequency analysis at the source level. This analysis was time-locked to the moments the Communicator and Salesman started and finished planning (epochs D: planning) and the Addressee and Roadworker started and finished observing (epochs E: observation), extending over a time window of 2.75 seconds (range: -0.5 to +2.25 and -2.25 to 0.5 seconds resp., resolution: $50 \mathrm{~ms}$ ). We applied an adaptive spatial filtering approach within a set of frequencies $(55-85 \mathrm{~Hz})$ shown to contain task-relevant neural activity by Analysis \#1. Here, $200 \mathrm{~ms}$ windows were tapered with 3 orthogonal Slepian tapers ( $\pm 10 \mathrm{~Hz}$ smoothing) prior to applying the Fourier transforms. Projection of the sensor-level data through the spatial filters, and subsequently computing the magnitude squared, yielded a location-specific (absolute) estimate of the time course of spectral power at the frequency of interest.

\section{Statistical model and inference}

We considered differential effects evoked during corresponding trial epochs in participants playing the Communicator or the Salesman role (epoch D: planning in Figure 1) and the Addressee or the Roadworker role (epoch E: observation). First, we estimated participant-specific effects (independent samples t-tests) on signal power at the source level (obtained from Analysis \#1) for each of these two sets of temporally-independent comparisons. Second, these participantspecific effects were z-normalized in order to account for differences in degrees of freedom and entered into a second-level random effects analysis correcting for multiple comparisons at the cluster level ( $\mathrm{p}<0.05 ; 10,000$ randomizations) (Maris \& Oostenveld, 2007). Third, the resulting group statistics of the two contrasts were entered into a conjunction analysis (Nichols, Brett, Andersson, Wager, \& Poline, 2005), effectively implementing a logical AND relation between the individual contrasts.

\section{Results}

We studied twenty-four pairs of participants engaged in real-time controlled interactions (de Ruiter, et al., 2010), and measured neural activity with MEG from one participant within each pair. Each pair of participants played an interactive game which requires the generation and understanding of novel, mutually negotiated communicative actions (i.e. communicative interactions between a "Communicator" and an "Addressee" pair, Figure 1, Movie 1). We distinguished neural activity specifically associated with those communicative actions from activity evoked during another interactive game that involved the same stimuli, responses, attention, and between-participants dependencies, but no communicative necessities (i.e. instrumental interactions between a "Salesman" and a "Roadworker" pair, Figure 1, Movie 2). Within each task, participants alternated between those two task-specific roles on a trial-bytrial basis (80 trials in each task). We further distinguished neural activity common to both generating (epoch D: planning, Figure 1) and understanding communicative actions (epoch E: observation, Figure 1) from activity uniquely evoked by either task component by means of conjunction analyses (Nichols, et al., 2005). An absolute index of neural activity was quantified by estimating ('beamforming') time-resolved spectral power of the signals recorded with MEG before and during task performance (Gross, et al., 2001).

The communicative and instrumental tasks are explained in detail in Appendix A of this chapter. Here we highlight their overlapping and differing features relevant for labeling and interpreting the results. In both tasks, pairs of participants were instructed to move their token on a visually presented $3 \times 3$ digital grid (Figure 1). In the communicative task, the goal of the 'Communicator' was to make sure that both his token (e.g. a circle) and that of the 'Addressee' (e.g. a triangle) were arranged according to a configuration visually presented to the Communicator only. This required the Communicator to use the movements of his token to indicate to the Addressee how she should configure her token on the grid. This task has proven effective in encouraging the generation of pair-specific communicative behaviors (Blokpoel, et al., 2012; de Ruiter, et al., 2010; Volman, Noordzij, \& Toni, 2012). The same movements could be used by different pairs to negotiate different meanings, and the same meaning could be conveyed by different movements across different pairs (Movie 3). The same movement could even be used to convey different goal states by the same pair in different trials (Movie 4), and vice versa (Movie 5). The latter observation emphasizes how, in this game, a movement acquires meaning by virtue of the history of the communicative interactions within a given pair, rather than by virtue of its sensory attributes. In the instrumental task, the goal 


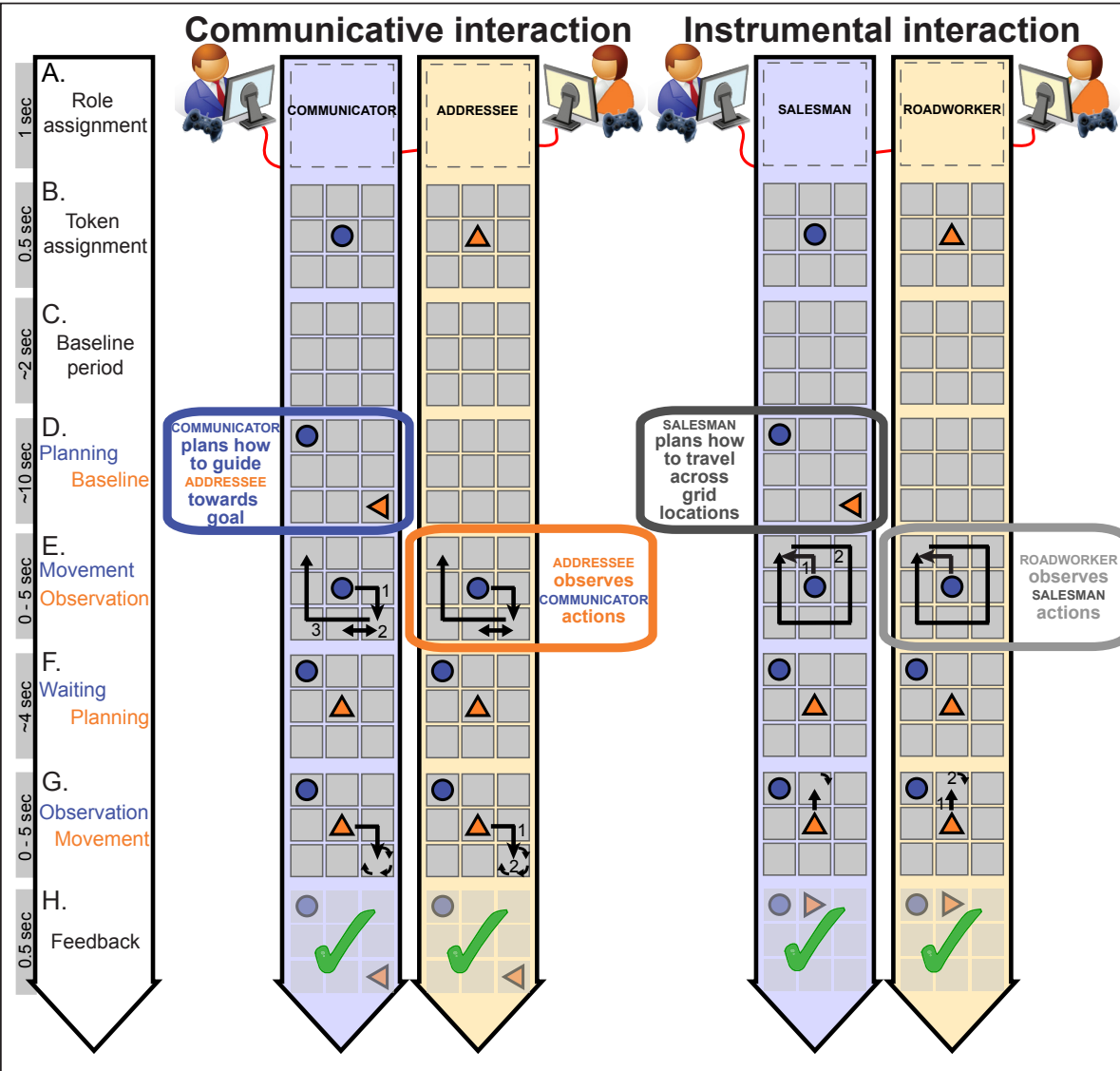

Figure 1. Live interactions. During a communicative interaction (see Movie 1), pairs of participants had to jointly reproduce a spatial configuration of two tokens shown only to the Communicator (epoch D). This required the Communicator to use the movements of his token (in blue, epoch E) to indicate to the Addressee how she should configure her token (in orange). In this game, shared meanings of the behaviors had to be constructed and inferred. During an instrumental interaction (see Movie 2) the same pair interacted by moving their tokens on the board according to pre-assigned rules. The Salesman implemented his rules on a visually presented configuration. The Roadworker implemented her rules according to the behavior of the Salesman in epoch E. The critical epochs for the analysis of neural activity are the planning phase (D) for the Communicator/ Salesman and the observation phase (E) for the Addressee/ Roadworker. of the 'Salesman' was to move his token across the board following a learned rule, according to a visually presented configuration. The co-player, labeled 'Roadworker', was instructed to place her token on the board following a learned rule, according to the movements of the Salesman on the board. Stimuli, movements, and between-player dependencies were matched between the two types of interactions, but the necessity to construct and infer shared movementmeaning mappings differed. In the communicative task, the success of a trial relied on the Communicator designing an action that can be understood by the Addressee, and on the Addressee inferring the Communicator's intentions. In the instrumental task, the success of a trial relied on each of the two players

\section{Behavioral characteristics of communicative interactions}

Participants solved both tasks well above chance level (communicative trials: $71 \pm 3 \%$ correct; instrumental trials: $73 \pm 4 \%$ correct, mean \pm SEM; Figure S1E; estimate of chance level: 1/32th, 8 locations with 4 potential orientations). The communicative interactions evoked stronger mutual adjustments between pairs than the instrumental interactions. First, during the communicative interactions, Communicators spent longer times at the grid location where the Addressee should place her token (Addressee 'target'), as compared to other visited locations on the board ('non-targets'; Location x Task interaction: $\mathrm{F}(1,23)=108.0, p<0.001$; Figure $\mathrm{S} 1 \mathrm{~F})$. This pausing behavior was adjusted to the inferred knowledge of the communicative partner on a trial-by-trial basis (Blokpoel, et al., 2012), a quantitative indication of recipient design (Newman Norlund et al., 2009). Second, during the communicative interactions, Communicators made repeated movements from and to the target location to indicate the desired orientation of the Addressee's token ( $2.09 \pm 0.49$ visits per trial, mean \pm SD; see action \#2 - epoch E in Figure 1, communicative task). Thi behavior was not observed in the instrumental task, and it follows the general principle of using a patently dysfunctional action to ostensively mark the action as being communicative in nature (Sperber \& Wilson, 2001). Third, in the communicative interactions, the within-trial coupling between Communicator and Addressee planning times ( $r=0.29 \pm 0.17$, z-transformed cross-correlations) was stronger than in the instrumental interactions (i.e. between Salesman and Roadworker planning times; $\mathrm{r}=0.09 \pm 0.22 ; \mathrm{t}(23)=4.2 ; p<0.001)$. This observation suggests that a difficult communicative problem was concomitantly more difficult for both Communicators and Addressees (de Ruiter, et al., 2010 van Rooij et al., 2011). Fourth, in the instrumental task, the number of executed movements explained a larger portion of planning time variance than in the communicative task (instrumental: $r=0.63 \pm 0.12$; communicative: $r=0.42$ $\pm 0.17 ; \mathrm{t}(23)=5.5, p<0.001)$. This finding suggests that, in the instrumental task, planning times increase almost linearly with an increasing number of 
movement steps to plan. In contrast, in the communicative task, planning times were governed by cognitive operations less directly related to the mechanics of the individual movement steps.

\section{Neural characteristics of communicative interactions - spatial} and spectral features

Having shown the relevance of the communicative task for studying novel communicative actions, we also verified that the neural activity evoked by performance of the communicative and instrumental tasks was largely matched (Figure S2), and devoid of eye movement confounds (Figure S3). Having satisfied these pre-conditions, we proceeded to test the three hypotheses of this study. First, we isolated neural activity evoked by the communicative task over and above the instrumental task, testing whether those neural differences were present in the sensorimotor system or in higher-order cortical areas. We considered the whole time interval covered by the planning and observation epochs (epoch D and E in Figure 1), a conservative approach that intrinsically focuses towards neural effects spanning both epochs. Two brain regions (right temporal lobe and ventromedial prefrontal cortex; Figure 2A and C) falling outside the core sensorimotor systems exhibited significantly stronger power over a broad frequency range (Figure $2 \mathrm{~B}$ and $\mathrm{D}$ ) during the processing of communicative actions than during instrumental actions. There were no significant clusters where planning or observing instrumental actions evoked stronger responses than communicative actions.

The second hypothesis of this study predicts an overlap in the cognitive processes evoked during generation and comprehension of novel shared symbols. Accordingly, we tested whether those task-dependent neural differences are shared between planning (epoch D) and observing (epoch E) communicative actions. We used a minimum-statistic conjunction analysis (Nichols, et al., 2005) to isolate neural effects shared across communicative roles, and different from the corresponding instrumental roles (Price \& Friston, 1997), effectively filtering out between-tasks differences that are not consistent across paired roles within each task (Figure S1C and D). The overlap in neural effects across communicative roles was statistically most pronounced in the $55-85 \mathrm{~Hz}$ gammaband (Figure S4) and spatially encompassed the ventromedial prefrontal cortex (vmPFC) and the right temporal lobe (TL; Figure 2E, in brown). 


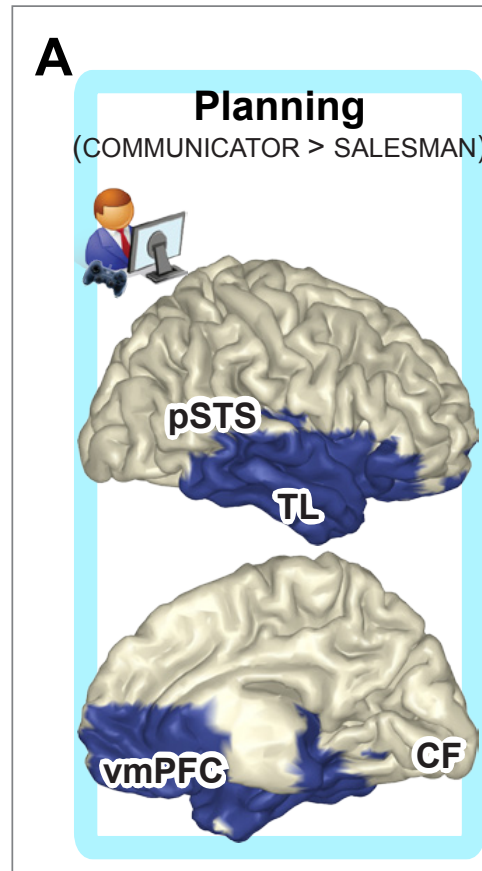

Communicative interactions Planning (COMMUNICATOR) Baseline (COMMUNICATOR)

\section{Instrumental interactions}

Planning (SALESMAN)

$\square$ Baseline (SALESMAN)
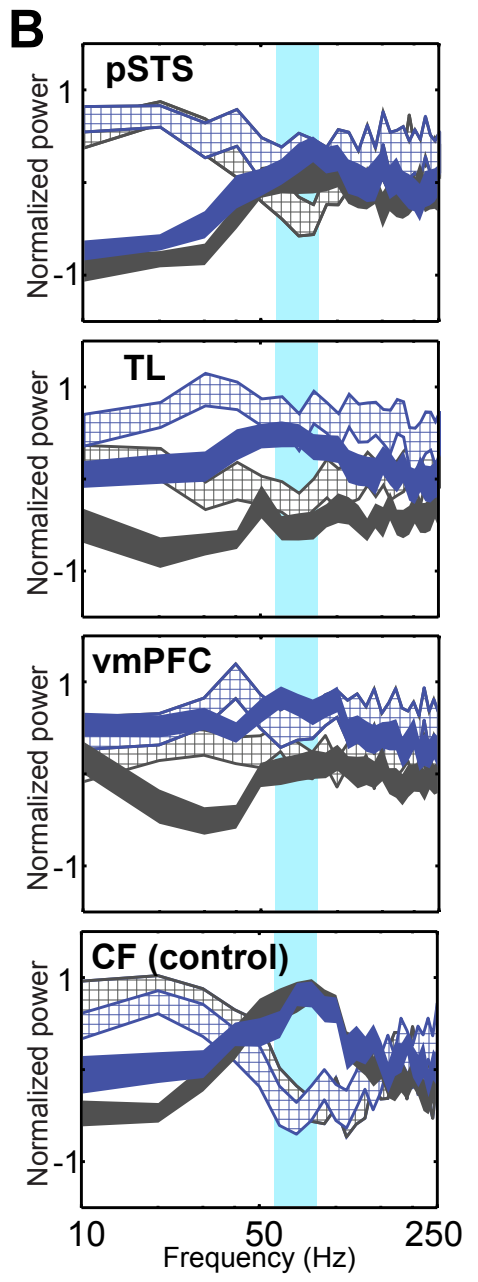

Figure 2. Spatial and spectral characteristics of neural activity evoked during communicative and instrumental interactions. Brain regions exhibiting stronger gamma-band activity $(55-85 \mathrm{~Hz})$ when participants planned (A) and observed (C) communicative action as compared to instrumental actions. The spatial distribution of the conjunction (E, in brown) was lateralized to the right hemisphere, covering most of the temporal lobe (TL) and ventromedial prefrontal cortex (VmPFC). Power spectral densities of neural activity ( +1 SEM,
C

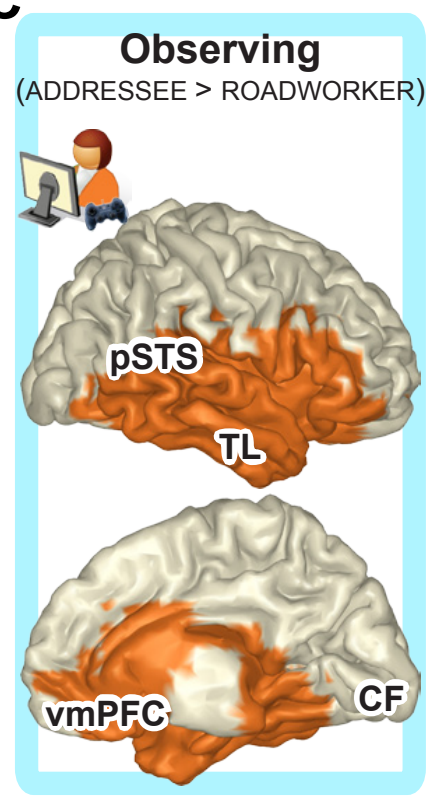

Communicative interactions Observing (ADDRESSEE)

Baseline (ADDRESSEE)

Instrumental interactions

Observing (ROADWORKER)

$\square$ Baseline (ROADWORKER)
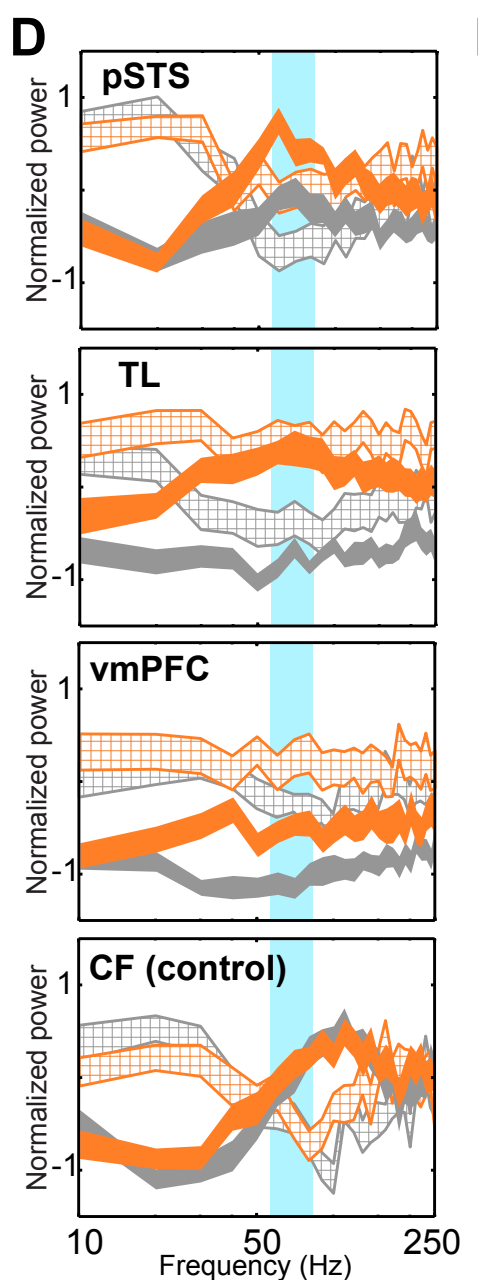

$E$

\section{Overlap}

(planning \& observing)

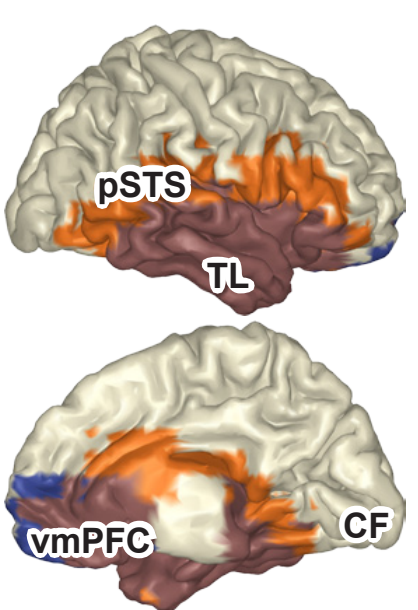

Left hemisphere

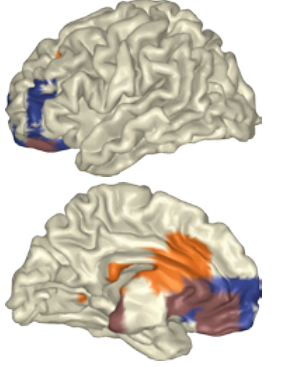

striped for baseline epochs, filled for task epochs; B and D) indicate that the task differences were broadband in nature (compare blue vs. dark grey and orange vs. light grey ribbons), statistically most pronounced in the $55-85 \mathrm{~Hz}$ frequency range (in cyan). Spectral power is mean-centered (i.e. the average across the eight experimental epochs was set to zero) and variance-normalized (SD $=1)$ over subsequent $10 \mathrm{~Hz}$ frequency bins. When averaged over the whole task epochs, the differences in the right posterior superior temporal sulcus (pSTS) were present only during the observation epoch (but see Figure 3). The calcarine fissure (CF) is presented for control purposes, showing only band-limited modulations from baseline. 
Neural characteristics of communicative interactions -

\section{temporal features}

The third hypothesis of this study predicts that selecting and understanding novel shared symbols relies on a cognitive set implemented through ongoing neural activity that predates the occurrence of the communicative stimulus material itself. Therefore, we explored the temporal dynamics of an absolute index of neural activity, i.e. source-reconstructed time-resolved estimates of gamma band power (Gross, et al., 2001). This index is appropriate for isolating tonic state-dependent effects that are temporally stable and not exclusively bound to the occurrence of task events. We observed upregulated neural activity in three regions (Figure 3). A ventrolateral portion of the right temporal lobe showed a tonic upregulation of gamma-band power during both planning and observation of communicative actions (TL, Figure $3 \mathrm{~A}$ and $\mathrm{B}$ ), without transient responses time-locked to the sensori-motor events occurring during those epochs. This temporal dynamics indicate that neural activity in the right temporal lobe is modulated by the communicative task, but over a timescale decoupled from within-trial events. A different neural dynamics was found in the ventromedial prefrontal cortex. This region showed a sustained decrease in gamma-band power during the observation epochs of both tasks, again with stronger gamma-band power in the communicative task, and a sharp power increase when participants started selecting their actions on the basis of the observed movements of their co-player (vmPFC, Figure 3). This temporal dynamics indicates that neural activity in the vmPFC is tonically upregulated during performance of the communicative task, with planning and observation of actions evoking opposite computational loads in this region with respect to the pre- and post-epoch phases. A third temporal profile of gamma-band activity was found in the right posterior superior temporal sulcus (pSTS), a region previously reported to increase its metabolic demands as a function of communicative difficulty, both for Communicators generating novel communicative actions and Addressees trying to decode those signals (Noordzij et al., 2009). Differently from the ventral portions of the right TL and the vmPFC, the right pSTS is sensitive to computational demands that occur early in planning and that rise during action observation (pSTS, Figure 3).

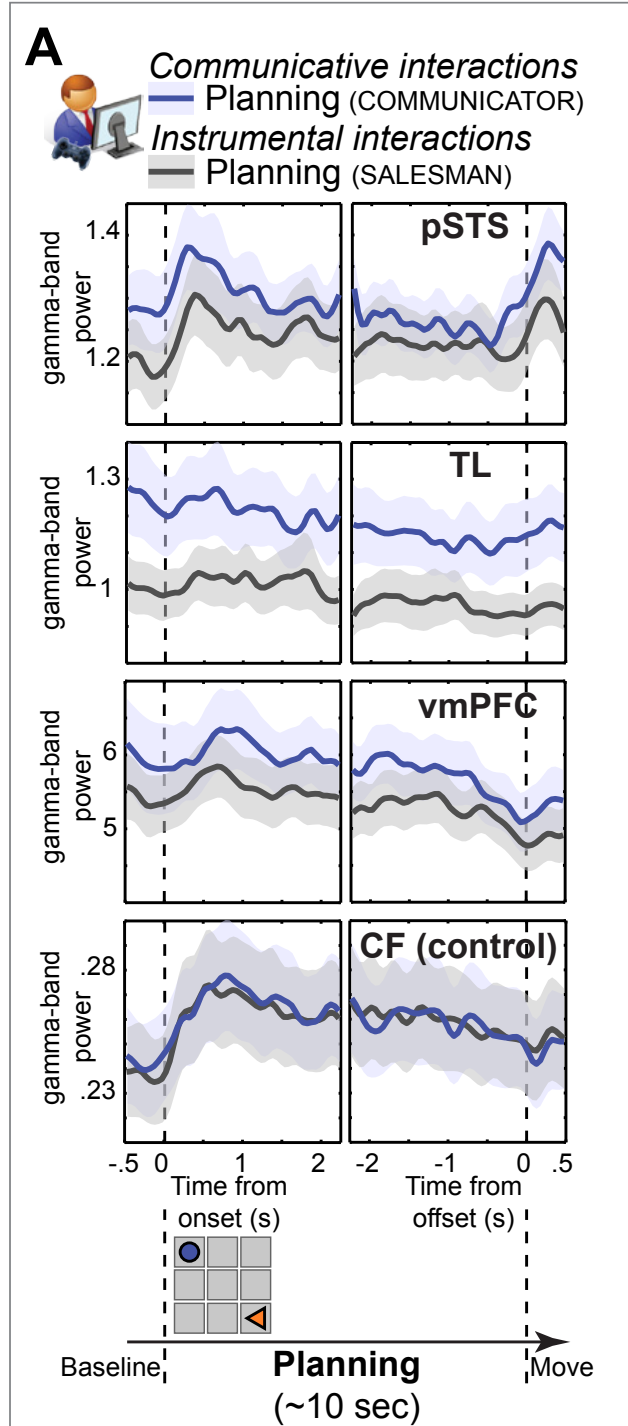

$B$ is

Communicative interactions - Observing (ADDRESSEE) Instrumental interactions
Observing (ROADWORKER)

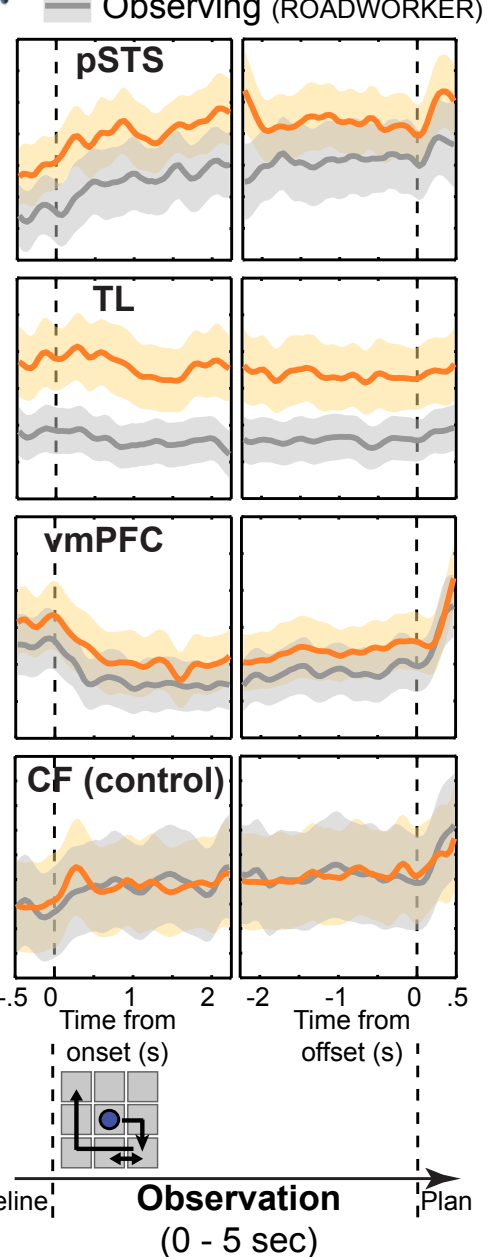

Figure 3. Temporal characteristics of neural activity evoked during communicative and instrumental interactions. Gamma-band activity $(55-85 \mathrm{~Hz}$, in arbitrary units \pm 1 SEM) in posterior superior temporal sulcus (PSTS), temporal lobe (TL), ventromedial prefrontal cortex (vmPFC), and calcarine fissure (CF), time-locked to planning (A) and observation (B) onset and offset. The graphic panel at the bottom highlights the characteristics of the relevant task epochs. Both tasks induced transient changes in neural activity in PSTS, vmPFC, and CF. During the communicative task neural activity was tonically upregulated in TL, VMPFC, and pSTS, but not in CF. 


\section{Communicative consequences of tonic upregulation of} gamma-band power

The gamma-band power changes described above were spatially and functionally specific, as illustrated by the absence of a tonic upregulation of gamma-band power during the same task epochs in a primary sensory area (the ipsilateral calcarine fissure, Figure 3 bottom row), despite strong transient changes in gamma-band power time-locked to the presentation of the visual stimuli. A fourth analysis tested whether those tonic gamma-band power changes are behaviorally relevant, with measurable consequences on the performance of the communicative task. We assessed trial-by-trial correlations of neural activity and behavioral performance (Appendix A). Gamma-band activity measured during the baseline period preceding the occurrence of observable events predicted the planning time of the subsequent trial epoch both when solving communicative and instrumental problems (both as Communicator/ Salesman, or as Addressee/ Roadworker). Critically, the spatial distribution and magnitude of the baseline neural activity predicting task performance differed as a function of the current cognitive set. During the communicative task, tonic baseline activity in the right temporal lobe (of both Communicator, epoch $\mathrm{C}$ in Figure 1; and Addressee, epoch D) predicted planning time in the same trial (Communicator: epoch D; Addressee: epoch F; $r=0.07 \pm 0.02 ; \mathrm{t}(23)=2.5, p$ $<0.03$; Figure 4A, C). The spatial distribution of this effect overlaps with the changes in gamma-band activity shared across the two communicators (Figure 2E). In contrast, during the instrumental task, tonic baseline activity in the parieto-occipital cortex (of both Salesman and Roadworker) predicted planning time in the same trial $(\mathrm{r}=0.09 \pm 0.02 ; \mathrm{t}(23)=3.5, p<0.05$; Figure 4B, C). The spatial distribution of this effect overlaps with the known contribution of the parieto-occipital cortex in supporting visuospatial transformations during action planning (Verhagen, Dijkerman, Medendorp, \& Toni, 2012), and with the observation that planning time during the instrumental task was linearly related to the number of movement steps performed by the subjects in the subsequent task epoch. In both tasks, there were no significant correlations between tonic baseline activity and planning time in the preceding trial $(\mathrm{r}=$ $0.02 \pm 0.02$ for each task).

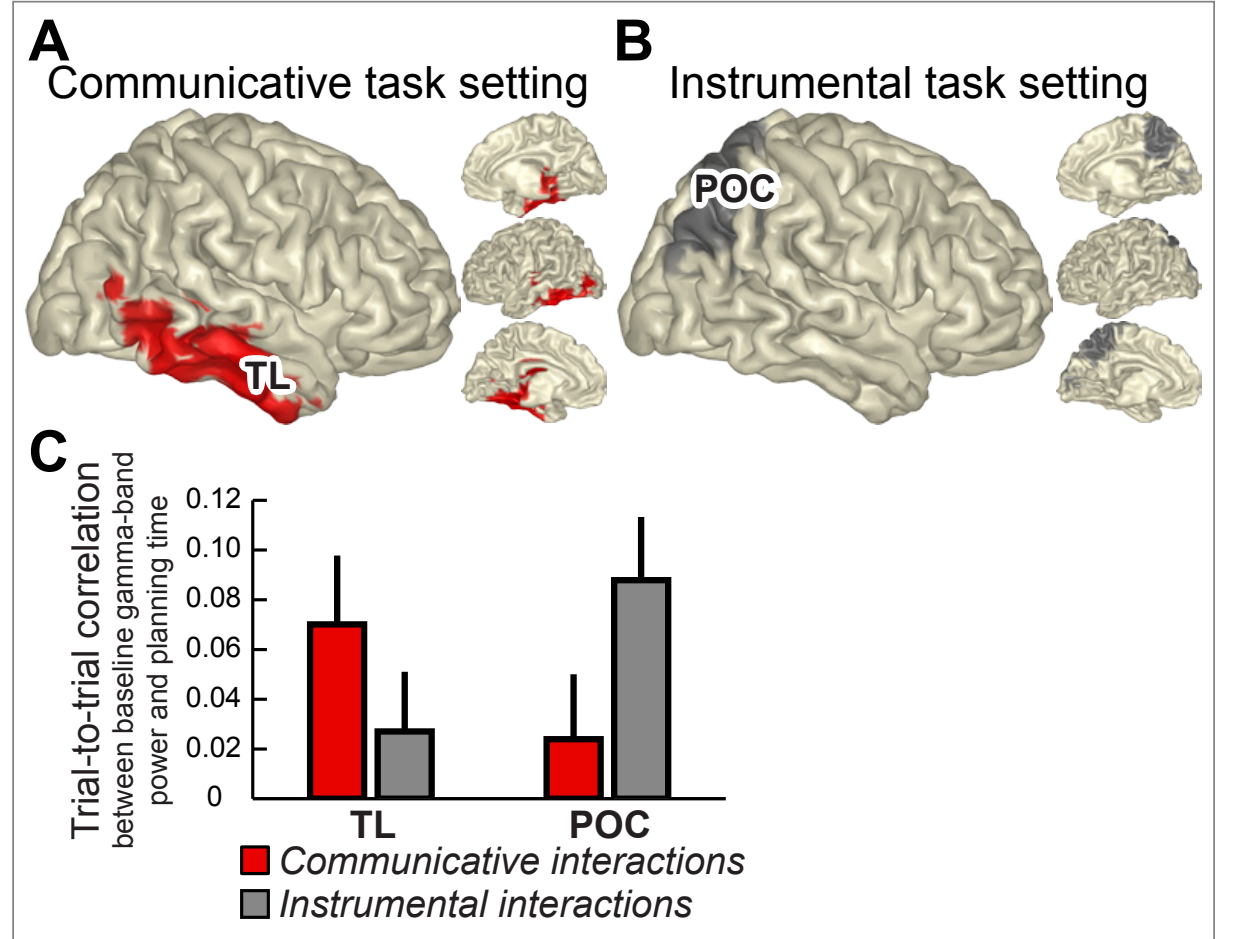

Figure 4. Ongoing neural activity associated with the cognitive set. (A and B) Spatial distribution of cortical regions showing trial-by-trial correlation between baseline neural activity and task performance. Baseline gamma-band $(55-85 \mathrm{~Hz})$ power in the temporal lobe (TL) accounted for variation in planning time of Communicators and Addressees; baseline gamma-band power in the parieto-occipital cortex (POC) accounted for variation in planning time of Salesmen and Roadworkers. (C) Groupaveraged correlations for each of the two tasks and cortical regions ( \pm 1 SEM). 


\section{Discussion}

This study describes the spectral, spatial, and temporal features of neural activity evoked during the selection and comprehension of novel shared symbols, two processes essential for understanding the flexibility of human communication (Levinson, 2006; Tomasello, 2008). There are three main findings. First, solving novel communicative problems upregulated local neural activity in the right ventrolateral temporal lobe and the ventromedial prefrontal cortex, two regions necessary for processing conceptual knowledge and mental models of other agents (Lambon Ralph, et al., 2010; Milne \& Grafman, 2001; Sabbagh, 1999). Second, the same upregulation of neural activity was found across Communicator and Addressee, irrespectively of whether a communicative action was being selected or comprehended. This finding indicates that the overlapping neural upregulation was driven by abstract task features shared across interlocutors, rather than sensorimotor events which differed between interlocutors. Third, the overlapping neural upregulation was present well before the occurrence of a specific communicative problem. This finding provides a neural counterpart to the notion that the meaning of novel communicative actions is inferred by embedding those stimuli in a conceptual space whose activation predates in time the processing of the communicative stimuli themselves (van Berkum, van den Brink, Tesink, Kos, \& Hagoort, 2008). Taken together, these observations indicate that the brain solves the computational challenges evoked by creating novel shared symbols by upregulating the same neuronal mechanism in the same brain regions across pairs of communicators, and over temporal scales independent from transient sensorimotor events (Hasson, Yang, Vallines, Heeger, \& Rubin, 2008).

\section{Tonically increased neural activity during communicative} interactions

The upregulation of neural activity evoked by the presence of communicative demands had specific spatial, spectral, and temporal characteristics. First, the spatial distribution of differential neural activity between the communicative and the instrumental task was confined to the right temporal and medial prefrontal regions. These two areas have been shown to be necessary for accessing conceptual knowledge and mental models of other agents (Lambon Ralph, et al., 2010; Milne \& Grafman, 2001; Sabbagh, 1999). Second, the spectral profile of this differential source-reconstructed neural activity was extremely broad. Physiologically, broadband shifts of local neural activity are functionally distinct from band-limited neuronal oscillations (Buzsaki \& Wang, 2012), and they are thought to reflect changes in mean firing rates of neuronal populations, see Appendix B of this chapter for an elaboration on this topic
(Buzsaki, Anastassiou, \& Koch, 2012; Manning, Jacobs, Fried, \& Kahana, 2009; Miller, 2010; Miller, Sorensen, Ojemann, \& den Nijs, 2009). Population-level firing rates have been shown to be affected by internal cortical states as much as by external stimuli (Arieli, Sterkin, Grinvald, \& Aertsen, 1996; Luczak, Bartho, \& Harris, 2013), and they are instrumental for integrating driving afferences with contextual information (Behabadi, Polsky, Jadi, Schiller, \& Mel, 2012; Jarsky, Roxin, Kath, \& Spruston, 2005; Larkum, 2012). Third, the temporal profile of the broadband shift of neural activity started already during the baseline epoch, before the presentation of a particular communicative problem, and well before the observation of communicative actions. This baseline-related local neural activity had measurable behavioral consequences on communicative performance during a subsequent epoch in the same trial (Figure 4), and it fits with the behavioral observation that these subjects displayed audience design during trials following a communicative error (Blokpoel, et al., 2012). Taken together, these observations suggest that the tonic upregulation of broadband neural activity evoked by communicative challenges reflects increased firing rates of neuronal populations in the right ventrolateral temporal lobe and the ventromedial prefrontal cortex. Those increased firing rates might provide a neurophysiological mechanism for integrating the current communicative problem with conceptual knowledge. Crucially, the present data suggest that this integration is not temporally bound to the presentation of a specific communicative problem in the course of a trial. In fact, the current findings support the notion that conceptual knowledge during a communicative interaction needs to be continuously aligned to the conversational context and to the interlocutor's behavior (Clark, 1996). The tonic upregulation of broadband activity observed in this study during communicative interactions might be a novel neural marker of this cognitive phenomenon.

\section{Shared tonic computations between production and} comprehension of communicative actions

A large portion of the right temporal lobe showed a sustained increase in broadband activity during both planning and understanding of communicative actions. This finding qualifies the characteristics of the coarse spatio-temporal cerebral overlaps between communicators reported in previous studies (Lerner, et al., 2011; Noordzij, et al., 2009; Schippers, Roebroeck, Renken, Nanetti, \& Keysers, 2010; Stephens, et al., 2010). Namely, the presence of a spectral overlap between communicators suggests that the human brain uses the same neurophysiological mechanisms when planning and understanding communicative actions. Given that those two epochs had considerable sensorimotor differences, and that the spectral overlap arose from brain 
regions necessary for processing conceptual knowledge and mental models of other agents, it is conceivable that Communicators and Addressees might share a basic conceptual mechanism that supports a rapid exploration through a large search space (Noordzij, et al., 2009).

\section{Shared phasic computations during social and non-social}

behaviors

This study shows that solving complex communicative and instrumental problems relies on computational processes with a surprisingly matched phasic neural dynamics. For instance, gamma-band power in the ventromedial prefrontal cortex transiently increased during the selection of complex action sequences, irrespectively of the communicative characteristics of those actions. The within trial fluctuations of gamma-band power in pSTS also showed a strikingly similar pattern when solving communicative compared to instrumental problems. These findings suggests that vmPFC and pSTS are involved in selecting communicative actions using neural dynamics similar to those involved in selecting non-communicative actions (Behrens, et al., 2009).

This observation argues against the notion that these two regions are exclusively dedicated to social cognition (Adolphs, 2009).

\section{Conclusions}

Humans are surprisingly effective at creating novel shared symbols (de Ruiter, et al., 2010; Galantucci, 2005), an evolutionary anomaly at the root of human communication (Levinson, 2006; Tomasello, 2008). This study describes the spectral, temporal, and spatial characteristics of neural activity evoked during planning and understanding of novel communicative actions. The computational challenges evoked by solving communicative problems result in tonically upregulated neural activity over right temporal and ventromedial prefrontal regions. The phasic temporal dynamics of those regions was sensitive to the occurrence of transient sensory or motor events, but it was indifferent to the communicative characteristics of the problems. These findings define the neurophysiological characteristics of a mechanism supporting human communicative innovation, opening the way for understanding the neural implementation of human symbolic communication. 


\section{Appendix A}

\section{Supporting Information}

\section{Experimental setting}

Each pair of participants engaged in two types of real-time sequential interactive tasks, a communicative task and an instrumental task - Figure 1 - with the order of presentation of the two tasks counterbalanced over participant pairs. The interactions between participants took place on a digital grid, visually presented and computer-controlled. Each participant controlled the movements of a token on the game board by means of a hand-held controller. Four buttons controlled by the right thumb moved the token to the left, right, up and down, respectively; the right shoulder button rotated the token 90 degrees clockwise; and the left shoulder button was used as a start- and end- button (panel A) During the experiment, one participant was supine on a bed inside a magnetically-shielded and sound-proof room. This participant was facing a projection screen and holding a MEG-compatible handheld controller (with the electrical wiring replaced by fiber optic cables - panel B). The visually presented digital game board subtended a visual angle of $\sim 2^{\circ}$ to minimize eye movements. The other participant sat outside the magnetically shielded room, in front of a 19 inch LCD monitor, using a structurally identical hand-held controller and wearing a sound-proof headset. An experiment lasted about three hours (see Experiment details below)

\section{Experiment details}

An experiment lasted about three hours with the following sequence of experimental sessions: preparation of the participants (delivery of instructions, placement of electrodes for electrocardiogram (ECG) and electrooculogram (EOG), $\sim 20$ minutes); training with using the hand-held controller $(\sim 15$ minutes); training in the first interactive game (20 trials, $\sim 20$ minutes); performance/recording of the first interactive game (80 trials, $\sim 45$ minutes); training in the second interactive game (20 trials, $\sim 20$ minutes); performance/ recording of the second interactive game ( 80 trials, $\sim 45$ minutes); acquisition of a MR anatomical scan ( $\sim 15$ minutes). Task events within each training and performance sessions were programmed using Presentation 9 (Neurobehavioral 
Systems, Albany, CA, USA) and run on a Windows XP personal computer handling visual presentation, receiving triggers from the hand-held controllers, and marking task events through triggers sent to the MEG acquisition system.

\section{Communicative interaction}

This task involves two players alternating between the roles of Communicator and Addressee across successive trials. At trial onset, each player is assigned a role and a token (left panel of Figure 1, epochs A and B: role and token assignment). After a baseline epoch consisting of an empty grid display (epoch C: baseline period), the Communicator (and the Communicator only) is shown the target configuration of that trial (epoch D: planning). The target configuration contains the tokens of the Communicator and the Addressee, at the grid locations and orientations they should have at the end of the trial. The Communicator knows that the Addressee does not see the target configuration, and that he cannot move the Addressee's token. Therefore, the Communicator needs to communicate to the Addressee the location and orientation that her token should have at the end of the trial. To comply with the task requirements, the Communicator also needs to ensure that at the end of his turn his token is at the location and orientation specified by the target configuration. In this game, the only means available to the Communicator for communicating with the addressee is by moving his own token around the grid, namely horizontal translations, vertical translations, or clockwise rotations. Both Communicator and Addressee also know that the Communicator has unlimited time available for planning his moves, but only five seconds for moving his token on the grid. The Communicator signals his readiness to move by pressing the start/stop button. At this point, the target configuration disappears, the Communicator's token appears in the center of the grid, and he can start moving his token (epoch E: movement). After five seconds, or earlier if the Communicator hits the start/stop button again, the Communicator's token cannot move further and the Addressee's token appears in the center of the grid. This event indicates that the addressee has acquired control over her token. The Addressee has unlimited time to infer the target location and orientation of her token on the basis of the observed movements of the Communicator (epoch F: planning). After the Addressee presses the start/stop button, she has five seconds to move her token (epoch G: movement). Finally, after five seconds, or earlier if the Addressee hits the start/stop button again, the same feedback is presented to both players in the form of a green tick or red cross (positive or negative feedback, respectively; epoch $\mathrm{H}$ : feedback). The feedback indicates whether the participants had matched the location and orientation of their tokens with those of the target configuration
Two important features of this communicative task should be emphasized. First, the Addressee cannot solve the communicative task by reproducing the movements of the Communicator's token. Rather, the Addressee needs to disambiguate communicative and instrumental components of the Communicator's movements, and find some relationship between the Communicator's movements and their meaning. Second, there are no a priori correct solutions to the communicative task, nor a limited set of options from which the Communicator could choose.

\section{Instrumental interaction}

In this task, two players alternated between the roles of Salesman and Roadworker across successive trials. At trial onset, each player is assigned a role and a token (right panel of Figure 1, epochs A and B: role and token assignment). After a baseline epoch consisting of an empty grid display (epoch C: baseline period), only the Salesman is shown the target configuration of that trial (epoch D: planning). The target configuration contains the tokens of the Salesman and the Roadworker. Differently from the communicative task, the target configuration of the instrumental task defines the trial-specific conditions of a problem that the Salesman needs to solve individually. Namely, the goal of the Salesman is to select a path of translations of his token through the grid, passing through a set of waypoints, in the following sequence: 1) starting position in the center of the grid (where the Salesman's token is placed at the end of the planning phase); 2) location of the Salesman's token as displayed in the target configuration (labeled as Salesman's "home" for clarity); 3) location and orientation of the token displayed in the target configuration that is different from the Salesman's token (labeled as the "outlet" for clarity); 4) location of the Salesman's home. The Salesman needs to satisfy a further requirement, namely he needs to pass exactly twice through one grid location different from the Salesman's home (that is also meant to be visited twice, see points 2 and 4 above). As in the communicative task, during the instrumental task the Salesman has unlimited time available for planning his moves, but only five seconds for moving his token on the grid. The Salesman signals his readiness to move by pressing the start button. At this point, the target configuration disappears, the Salesman 's token appears in the center of the grid, and he can start moving his token (epoch E: movement). After five seconds, or earlier if the Salesman hits the start button again, the Salesman's token cannot move further and the Roadworker's token appears in the center of the grid. This event indicates that the Roadworker has acquired control over her token. Similarly to what happened for the Addressee in the communicative task, the task of the Roadworker in the instrumental task depends on the movements of the co-player (i.e. Salesman). However, differently from the communicative task, in the instrumental task the Roadworker uses 
inadvertently displayed features of the Salesman's movements to solve her task. Namely, the Roadworker is asked to move to the grid location visited twice by the Salesman, excluding the Salesman's house. The Roadworker has unlimited time to decide where to move her token on the basis of the observed movements of the Salesman (epoch F: planning). After the Roadworker presses the start button, she has five seconds to move her token (epoch G: movement). Finally, after five seconds, or earlier if the Roadworker hits the start button again, feedback is presented to the two players in the form of a green tick or red cross (positive or negative feedback, respectively; epoch H: feedback). The feedback indicates to each player independently whether they had complied with the requirements of the instrumental task on that trial.

\section{Manipulations of task difficulty}

In both the communicative and instrumental task, we increased task difficulty across successive trials (for examples see Figure S1A and B). In the communicative task, the rationale of this intervention was to drive participants to continuously create new communicative behaviors, rather than exploiting already established communicative conventions. Communicative task difficulty was increased by introducing deliberate mismatches between the geometrical characteristics of the tokens of Communicators and Addressees. For instance, when the Communicator's token was a circle and the Addressee's token was a triangle (Figure S1A, middle column), then the Communicator needed to find a new way to indicate to the Addressee the orientation of her token, since rotations of the circle token were not visible. A further level of difficulty could be introduced by using a triangular token pointing outward the grid as the Addressee's target configuration, the Communicator's token being a circle (Fig S1A, right column).

In the instrumental task, the rationale was to match the surface behavior evoked in the communicative task. Instrumental task difficulty was also increased by introducing triangular shaped tokens for the Roadworker (the "outlet"). Outlets with a triangular token required the Salesman to leave the outlet along the direction to which the token was pointing and to enter it from any but the same side ('one-way rule', Figure S1B, middle column). A further level of difficulty could be introduced by using a triangular token as the Salesman's home since then the same rule would also apply to that location (Figure S1B, right column). Triangular tokens would also increase task difficulty for the Roadworker. Namely, if the Roadworker's token was a triangle, her task would then involve rotating her token such that the triangle pointed to the direction of the movement of the Salesman's token when it left that re-visited location the second time.

\section{Behavioral data analysis}

We considered mean planning times, mean movement times, and mean number of moves of Communicator and Addressee in the communicative task, and Salesman and Roadworker in the instrumental task (see Figure S1). These dependent variables were calculated for each of the twenty-four pairs of participants and for each of the two tasks, and compared statistically by means of paired t-tests (two-tailed $\alpha$-level $=0.05$ ). We also compared the mean time spent on target locations and on non-target locations (within movement epochs E - Figure 1) separately for each game, in a 2-way ANOVA with task setting (communicative, instrumental) and location (target, non-target) as factors. In the communicative task the target refers to the Addressee's target location that had to be communicated by the Communicator. In the instrumental task the target was defined as the location that had to be visited twice by the Salesman and reached by the Roadworker. Non-target locations were defined as the other grid locations visited by the Communicator or by the Salesman. We considered pairs of participants as the unit of observation for the statistical analysis since in the communicative task performance is dependent of both elements of a pair, and for consistency we adopted the same approach with the instrumental task. Finally, we considered the percentage of correct trials achieved by the participants in the communicative and instrumental task. Given the tasks characteristics, correct outcome could be defined on the basis of individual performance in the instrumental task, but only on the basis of joint performance in the communicative task. Accordingly, we refrained from directly comparing performance between the two tasks.

\section{MEG source reconstruction}

Participant-specific anatomical MRIs were used to linearly transform a 3-dimensional template grid (10 mm spacing) in MNI coordinates (MNI; Montreal Neurological Institute, Montreal, Quebec, Canada) to the coordinate system specific to the participant's head. To this end we used SPM8 (Statistical Parametric Mapping; http://www.fil.ion.ucl.ac.uk/spm) to estimate the affine transformation between the two coordinate systems. We subsequently applied the inverse of this transformation to obtain grid positions at matched brain locations across participants. For each of the positions on that grid, neural activity was estimated using a frequency-domain 'beamforming' approach This method constructs spatial filters for each of the grid positions, passing the activity from the location of interest with unit gain, while maximally suppressing activity from all other possible sources of neural and non-neura electrical activity. The beamformer spatial filter is constructed from the lead field and the cross-spectral-density matrix of the data. The lead field is the 
physical forward model of the field distribution calculated from an assumed source at a given location and the participant-specific volume conduction model of the head. Here, we used a single-shell volume conduction model of the brain, based on the brain boundary determined by the segmented anatomical MRI, and computed the leadfields according to (Nolte, 2003). In this study, we considered spatial filters generated by using condition- and participant- specific lead fields. This approach takes into account and controls for differences in head position and orientation of the sources relative to the MEG sensors, leading to more consistent and less biased estimates of source-level effects across participants and conditions.

\section{General assessments of neurophysiologic data}

The participant's head position relative to the MEG sensors was measured before, during, and after each session using localization coils, placed at the nasion and the left and right ear canals. Before the second session, each participant was asked to reposition his/her head in the same location and orientation as the position measured before the first session, using a real-time head localizer tool (Stolk, Todorovic, et al., 2013). To test for systematic differences in head positions, we computed the difference in the position of the center of the head between the two sessions for all participants. The average position difference along the axis accounting for most of the variance was $0.6 \pm 0.4 \mathrm{~mm}$ (mean \pm SEM), an indication of strong inter-session consistency in head location. Electrocardiogram traces (ECG), and vertical and horizontal electrooculogram traces $\left(\mathrm{EOG}_{\mathrm{v}}\right.$ and $\mathrm{EOG}_{\mathrm{h}}$ ) were recorded during task performances, using 3 pairs of $10-\mathrm{mm}$ diameter $\mathrm{Ag}-\mathrm{AgCl}$ surface electrodes with bipolar montages. The ECG showed no statistically significant differences in heart rate; communicative setting $69.2 \pm 1.8 \mathrm{bpm}$ versus instrumental setting $69.5 \pm 2.1 \mathrm{bpm}$ (mean \pm SEM). The EOG traces showed no statistically significant differences in overal signal energy: Communicator vs. Salesman, $\mathrm{t}(23)<0.8$; and Addressee vs. Roadworker, $\mathrm{t}(23)>-1.4$ (paired samples t-tests). To provide a more stringent filter against the effects of eye movements on the spatial distribution of taskrelated effects and on the temporal dynamics of source-reconstructed activity, we directly removed continuous eye movement estimates from the sourcereconstructed MEG data prior to further analyses. The contribution of vertical $\left(E O G_{v}\right)$ and horizontal $\left(E G_{h}\right)$ electro-oculographic signals was estimated in the same time segments and frequency bands as that of the source-reconstructed signal, and removed from that signal, according to the following procedure: where $\mathrm{Y}$ is source data over $\mathrm{K}$ trials, $\mathrm{b}$ is the intercept constant, $\mathrm{C}$ is a $\mathrm{K}$ vector of ones, $b_{1-2}$ are regression coefficients for eye movement related activity recorded at the vertical and horizontal EOG channels respectively. $\mathrm{E}$ is unexplained model error. The least squares solution to the linear equation,

$$
\min \left\|\mathrm{Y}-\mathrm{b}_{0} \mathrm{C}-\mathrm{b}_{1} \mathrm{EOG}_{\mathrm{v}}-\mathrm{b}_{2} \mathrm{EOG}_{\mathrm{h}}\right\|^{2}
$$

then results in three $b$ values per voxel (two for the EOG channels, and one constant). Subsequently, the estimated contributions of the EOG regressors to the source reconstructed spectral power was removed from the original singletrial source data:

$$
\mathrm{Y}_{\text {clean }}=\mathrm{Y}-\mathrm{b}_{1} \mathrm{EOG}_{\mathrm{v}}-\mathrm{b}_{2} \mathrm{EOG}_{\mathrm{h}}
$$

where $\mathrm{Y}_{\text {cean }}$ represents the data with eye movement related variance removed (and with the intercept constant remaining in the data). Supplemental Figure 3 illustrates the spatial distribution of beta values estimated for each EOG channel (vertical and horizontal) and epoch type (planning and observation of actions). It can be seen that the EOG signal in the $55-85 \mathrm{~Hz}$ band was significantly correlated with source-reconstructed activity from orbitofrontal cortex, most likely due to both locations picking up activity of the extra-ocular muscles involved during saccades (Carl, Acik, Konig, Engel, \& Hipp, 2012; Keren, Yuval-Greenberg, \& Deouell, 2010).

\section{Trial-by-trial coupling between baseline neural activity and} task performance

The source level trial-by-trial gamma-band powers were ensured free from head movements (Stolk, Todorovic, et al., 2013) prior to computing trial-bytrial correlations between gamma-band activity and planning times in a subsequent trial epoch. The planning times (i.e. Communicator/Salesman and Addressee/Roadworker in trial epochs D and F resp.) were log-transformed and both dependent variables were normalized per task role separately before concatenation (with equal number of trials per interaction type) and subsequent correlation. The significance of the coupling was tested by testing the z-transformed single-subject correlations against null at the group-level.

$$
\mathrm{Y}=\mathrm{b}_{0} \mathrm{C}+\mathrm{b}_{1} \mathrm{EOG}_{v}+\mathrm{b}_{2} \mathrm{EOG}_{h}+\mathrm{E}
$$


Supplemental Figure 1. Representative examples (A and B) and summar statistics (C-F) of interactive behaviors in the communicative and instrumental tasks. (A, B) Each column shows representative examples of interactive behaviors at three different levels of task difficulty, separately for communicative interactions (panel A) and instrumental interactions (panel B). The first row describes the initial problem faced by the Communicator (panel A) and by the Salesman (panel B). This task epoch

A
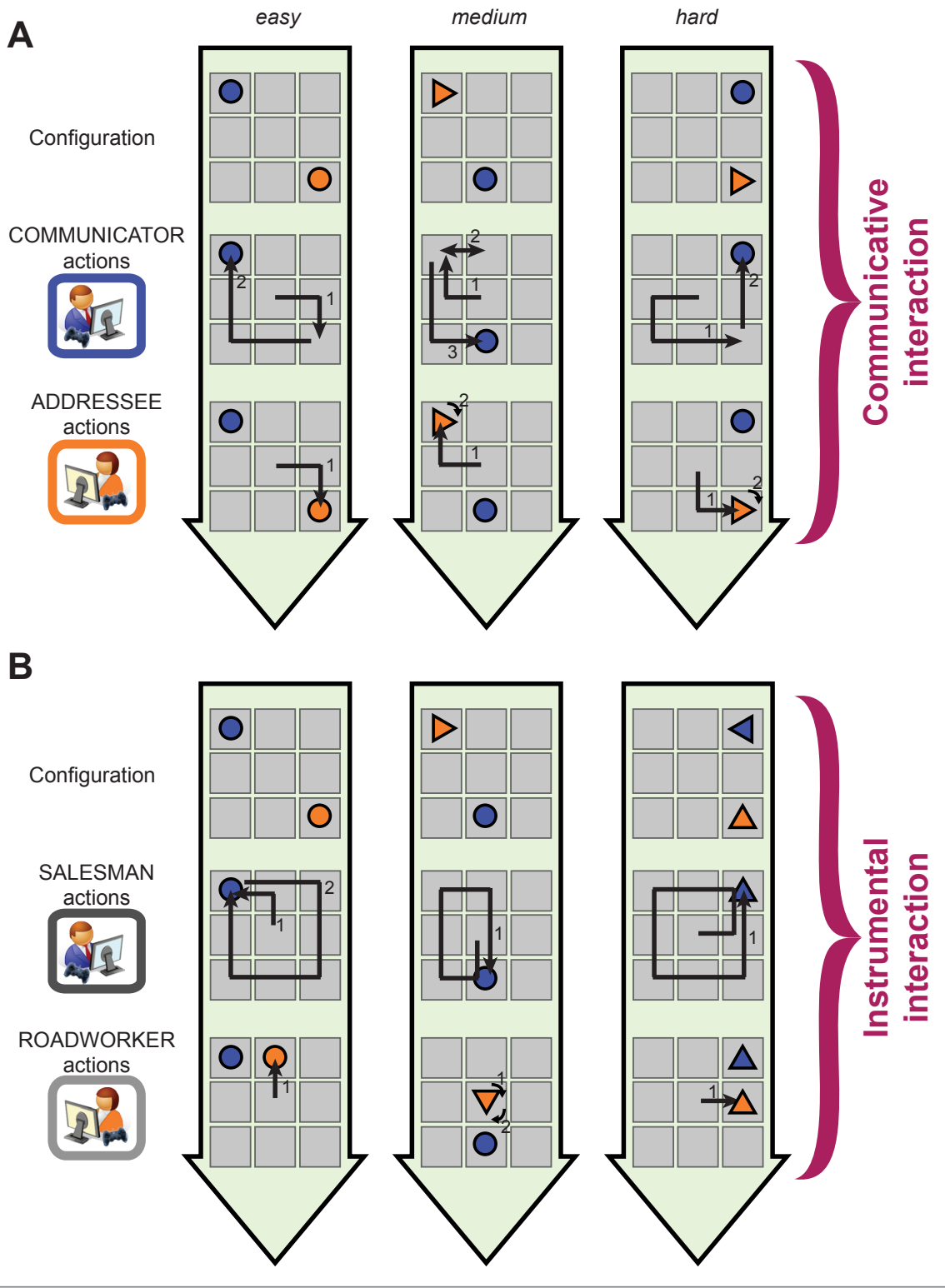

corresponds to epoch D in Figure 1. The second row in each panel describes the actions of the Communicator/Salesman (see epoch E in Figure 1), i.e. horizontal/vertical translations (arrows), sequences of translations (broken arrows), return translations (double arrows), and 90 degree clockwise rotations (small curved arrows). The third row in each panel describes the actions of the Addressee/Roadworker (see epoch H in Figure 1). On the next page we provide an account of some frequently observed interactive behaviors.

C
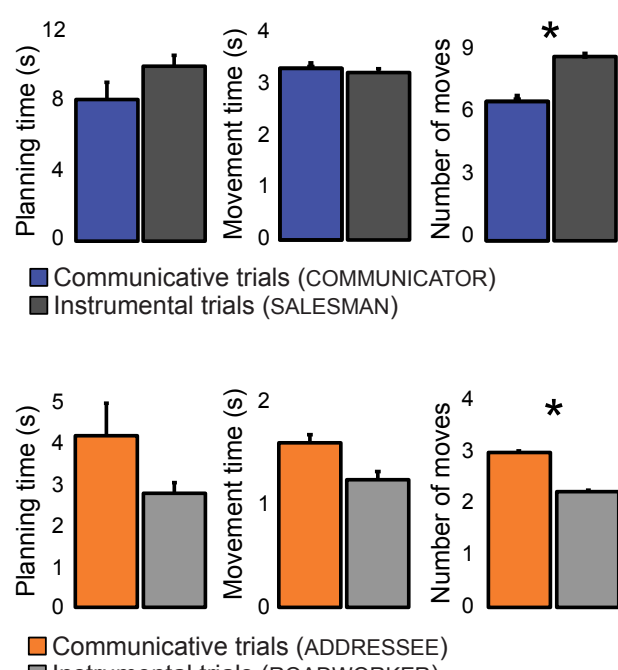

Communicative trials (ADDRESSEE)

$E$

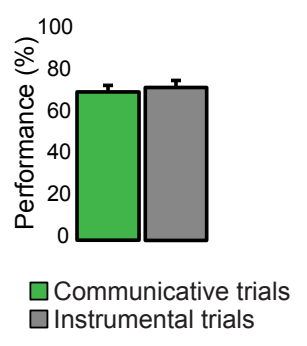

$\mathbf{F}$

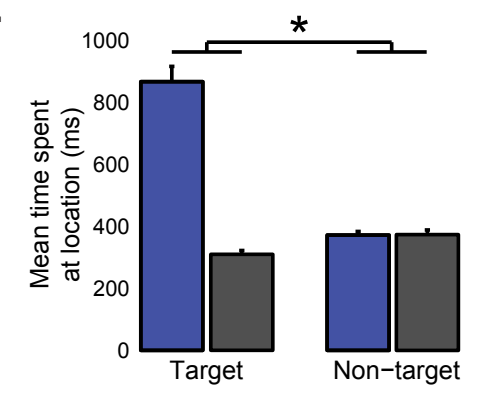

(C) Planning times (epoch D in Figure 1), Movement times (epoch E in Figure 1), and Number of moves of Communicators and Salesmen. Note that the Communicator and Salesman Movement times determine the Addressee and Roadworker observing times. (D) Planning times (epoch $\mathrm{F}$ in Figure 1), Movement times (epoch G in Figure 1), and Number of moves of Addressees and Roadworkers. Note that Addressees make more moves than Roadworkers, whereas Communicators make fewer moves than Salesmen. Therefore, task-related differential effects common to Communicators and Addressees (Figure 2E) cannot be driven by these behavioral differences in task performance. (E) Percentage of successful trials in the communicative and instrumental task. Note that, in the communicative task, successful performance is conditional on both players (green bar); the same parameter is provided for the instrumental task (grey bar). (F) Mean time spent at grid locations within the movement intervals, separately for target and non-target locations (in each case the average per trial is taken). In the communicative trials, target refers to the Addressee's target grid location that had to be communicated by the Communicator. For the instrumental trials, target refers to the location that was meant to be visited twice by the Salesman. The non-target locations refer to other visited locations on the digital grid. Error bars indicate \pm 1 SEM. *, $p<$ 0.001 . 


\section{Supplemental Figure 1 continued.}

Communicative interaction - Easy: The Communicator moves towards the addressee's target grid location (orange token), pauses, then moves his token to the own target location (blue token). The pause is dysfunctional to the communicator's goal of reaching his target. The Addressee infers that this instrumentally dysfunctional behavior performs a communicative function, marking the location that her token should have on the grid.

Communicative interaction - Medium: The Communicator moves towards the Addressee's target grid location, pauses, then moves one grid location along the direction the triangle is pointing to, moves back to the Addressee's target location, pauses again, and then moves to the own target location. The 'wiggling' signal (i.e. moving one grid location aside and back, depicted by the double arrow) is a more complex instrumentally dysfunctional behavior that assumes a communicative value, providing the addressee with an indication for the orientation that her token should have on the grid.

Communicative interaction - Hard: The Communicator makes a detour before going towards the addressee's target location, pauses at the Addressee's target location, and then goes to the Communicator's own target location. Marking the orientation of a token pointing outwards on the grid cannot be mapped to the communicative behaviors described above. Communicators solve this problem by exploiting the conversational context set by the previous examples, namely avoiding to produce wiggles, and marking this absence with an instrumentally dysfunctional detour. The absence of an orientation signal (the "wiggles"), together with an ostensive cue marking the salience of that absence (the detour), provides a new communicative signal that is interpreted as indicating a token orientation that cannot be marked by the "wiggle" strategy. Please note that this is only one among a series of possible solutions. For instance, some Communicators use the number of subsequent "wiggles" to mark the number of clockwise rotations that the Addressee needs to make to achieve the target orientation of her token.

Instrumental interaction - Easy: The Salesman moves towards the salesman's "home" (blue token) and returns to the grid location he came from which he now visited twice. He then moves towards the "outlet" (orange token) and subsequently to his "home" again, while avoiding re-visiting another grid location. There are three alternative solutions of which two include the re-visiting of the center left grid location instead. The Roadworker moves towards the grid location visited twice by the Salesman conform her objective ('repairing' the grid location visited twice by the Salesman).

Instrumental interaction - Medium: The Salesman visits the home location, then the outlet while obeying the one-way rule associated with the triangle's orientation (a triangular token required the salesman to leave that grid location along the direction to which the token was pointing and to enter it from any but the same side), and subsequently moves towards the home while re-visiting the center field (the star at the center grid counts as one visit to this location). The Roadworker stays at the center grid but rotates her token such that the triangle points to the direction of movement of the Salesman's token when it left that re-visited location the second time, conform her objective (see Manipulations of task difficulty in the SI Materials and Methods).

Instrumental interaction - Hard: While the Salesman now has to move along the one-way rules of two tokens, there is only one solution to his problem. The Salesman moves to the right so he can enter and leave his home along the direction of the triangle's point, and then moves around the grid towards the outlet while not revisiting any other grid location. He subsequently enters and leaves the outlet along the direction the triangle is pointing to, re-visits a previously visited grid location for the first time before returning home. The Salesman does not need to match any orientation with his own token. The Roadworker's token is triangular shaped and, therefore, needs to match the Salesman's movement direction (similar to the Medium example). However, since her token's orientation at start already matches the target orientation of this trial, she moves towards the Salesman's re-visited location without making any additional rotations. 

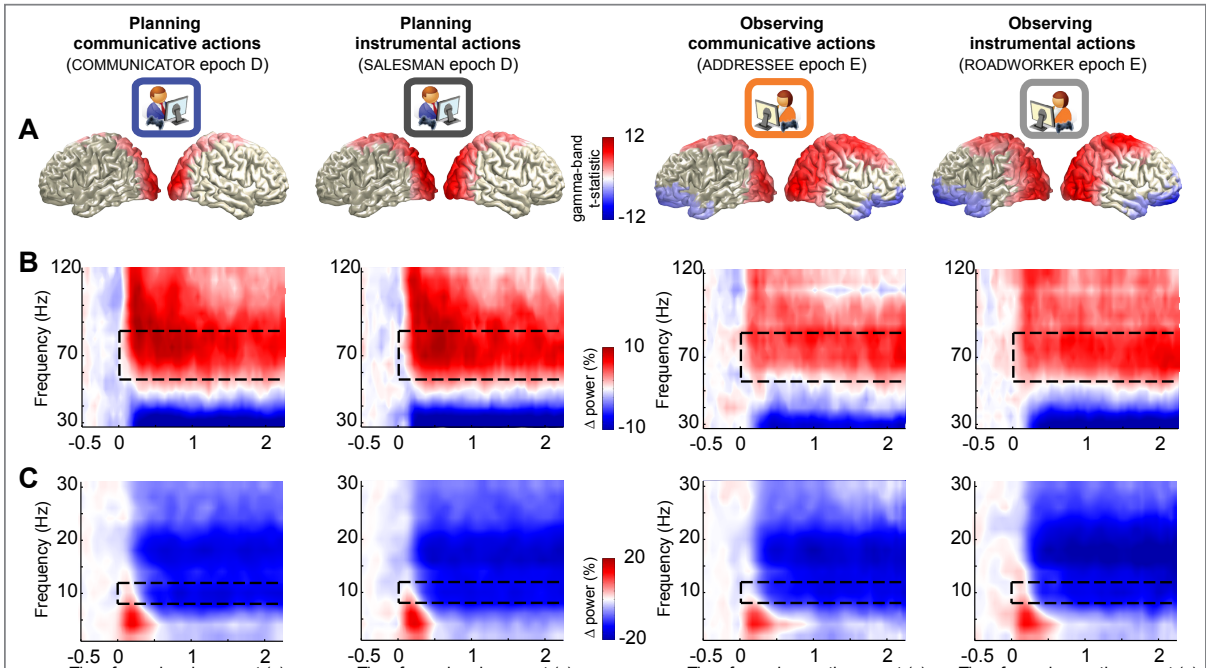

$\int_{-20}^{20}$
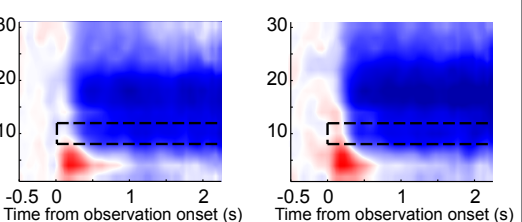

Time from planning onset (s)

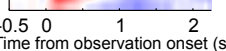

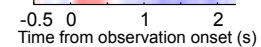
C.
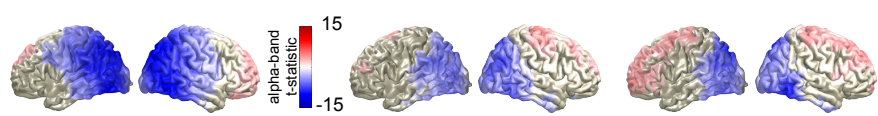

Supplemental Figure 2. Spatial, spectral, and temporal profile of task-related neural activity (A-D). The task evoked modulations in signal power (relative from baseline) indicate highly comparable patterns of induced neural activity in the sensorimotor system (occipital and posterior parietal cortex) within the two planning epochs (Communicator and Salesman; first and second column) and within the two observation epochs (Addressee and Roadworker; third and fourth column). The top two rows (A and B) represent the spatial, temporal, and spectral characteristics of changes in high frequency power $(>30 \mathrm{~Hz})$ evoked by the task. This analysis was based on $200 \mathrm{~ms}$ windows tapered with a set of 3 orthogonal Slepian tapers. The bottom two rows (C and D) represent similar characteristics of changes in low frequency power $(<30 \mathrm{~Hz})$ evoked by the task. This analysis was based on 500 ms windows and a single Hanning taper. (A) Lateral views on functional source reconstructions of gamma $(55-85 \mathrm{~Hz})$ activity evoked during the whole of the planning and observation epochs contrasted with the endmost second of their preceding baseline periods. (B) The power responses resolved in time and frequency in voxels that survived the multiple comparison statistics as a positive cluster in A $(p<0.05)$. (C) The power responses resolved in time and frequency in voxels that survived the multiple comparison statistics as a negative cluster in $\mathrm{D}(p<0.05)$. (D) Lateral views on functional source reconstructions of alpha $(8-12 \mathrm{~Hz})$ activity evoked during the whole of the planning and observation epochs contrasted with the endmost second of their preceding baseline periods.

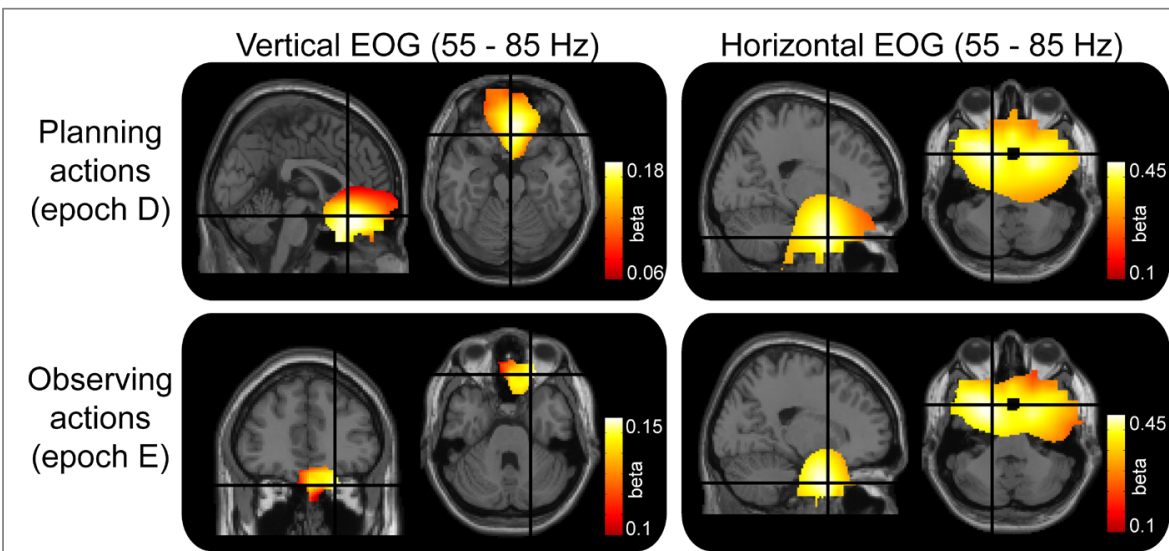

Supplemental Figure 3. Contributions from eye movement during the planning (upper row) and observation of actions (bottom row) were estimated and regressed out from the source-reconstructed data prior to further analysis. The normalized betaweights (obtained by normalizing the source and EOG data prior to multiple linear regression analysis) reveal the spatial structure of source-reconstructed activity, i.e. around the extra-ocular muscles, that is significantly correlated with vertical and horizontal EOG activity in the $55-85 \mathrm{~Hz}$ frequency range. The threshold of the color axis was raised in order to resolve the spatial structure around the statistically significant peaks (t-value $>8, p<0.05$, multiple comparison corrected). The upper beta-values are the peaks. 

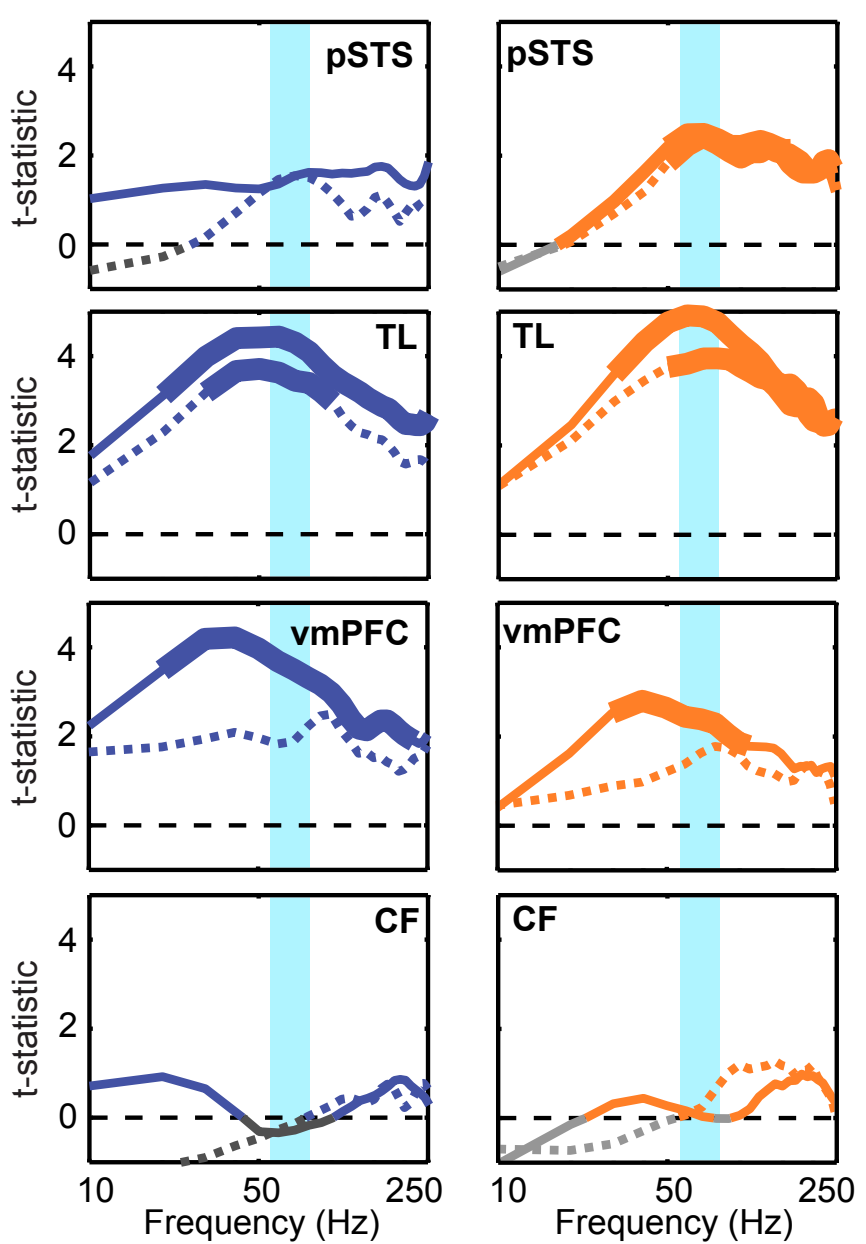

\begin{tabular}{|cc|}
\hline \multicolumn{2}{|c|}{ t-statistics, bold when $p<0.05$ corrected } \\
COMMUNICATOR vs. SALESMAN & ADDRESSEE vs. ROADWORKER \\
Planning & Observing \\
\hline & Baseline
\end{tabular}

Supplemental Figure 4. The t-statistics per frequency bin indicate that the differences in (absolute) neural activity between the communicative and instrumental task epochs were statistically most pronounced in the $55-85 \mathrm{~Hz}$ gamma-band (in cyan). The panels follow the presentation order of the power spectral densities in Figure 2B and D. The solid lines represent the t-statistics derived from group-level paired t-tests on source-reconstructed cerebral neural activity evoked during the whole of the planning and observation epochs. The dashed lines represent the same contrasts, but now regarding the endmost second of preceding baseline periods during which only the empty grid was presented.

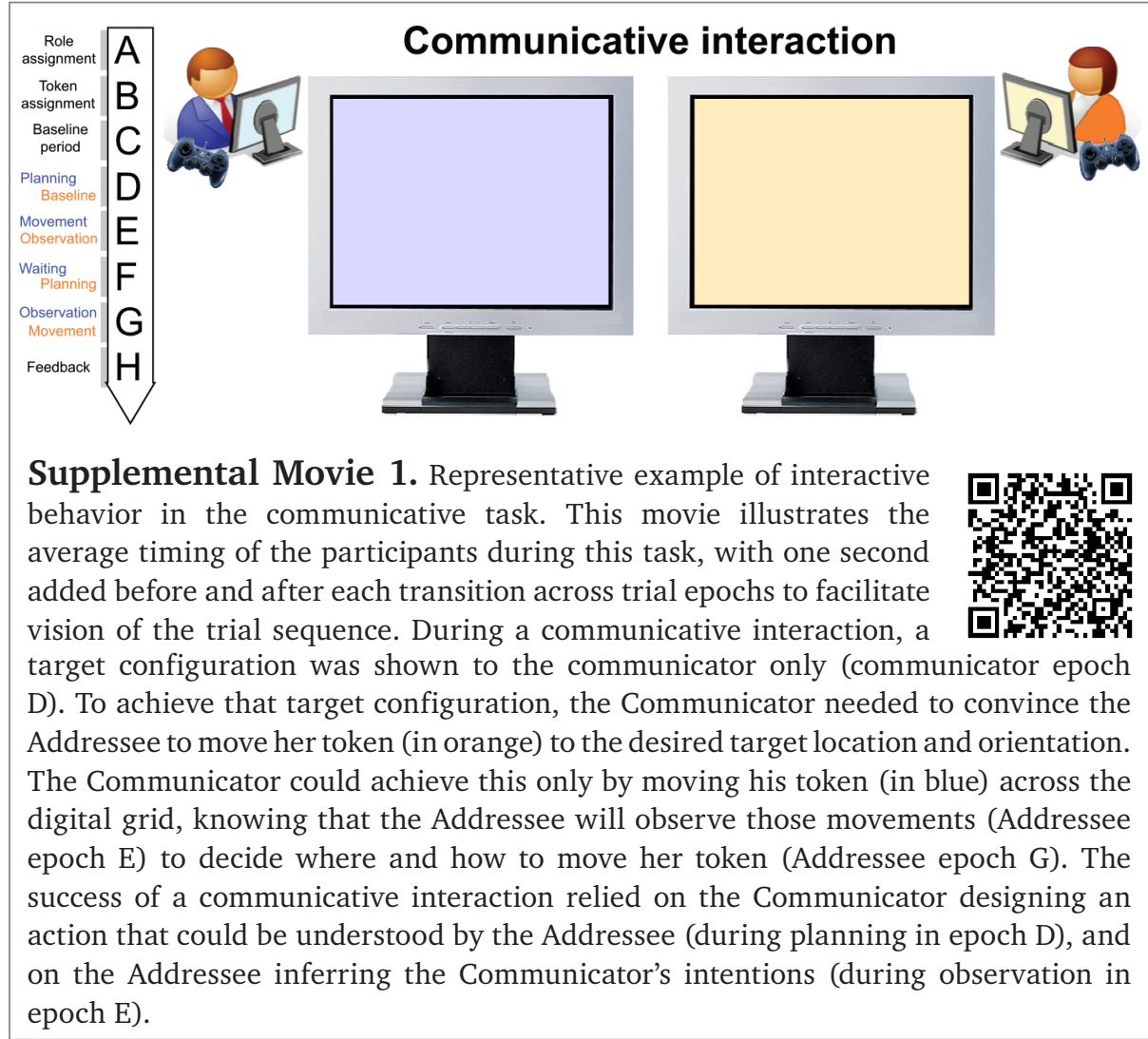

Supplemental Movie 2. Representative example of interactive behavior in the instrumental task. This movie illustrates the average

timing of the participants during this task, with one second added

before and after each transition across trial epochs to facilitate vision of the trial sequence. During an instrumental interaction, the Salesman's objective was to travel between two grid locations while visiting only one grid location twice (Salesman epoch D), knowing that the Roadworker will observe those movements (Roadworker epoch $\mathrm{E}$ ) to decide where and how to move to the grid location visited twice (Roadworker epoch G). A triangular token required the Salesman to leave that grid location along the direction to which the token was pointing and to enter it from any but the same side ('one-way rule'). Concomitantly, it required the Roadworker to rotate her token such that the triangle pointed to the direction of the movement of the Salesman's token when it left the re-visited location the second time. The success of an instrumental interaction relied on the Salesman designing an action according to pre-established rules (during planning in epoch D), and on the Roadworker implementing her assigned rules according to the behavior of the Salesman (during observation in epoch E). 
Supplemental Movie 3. Movies 3, 4, and 5 reproduce exactly the behavior of the participants recorded during the trials on display, with one second added before and after each transition across trial epochs to facilitate vision of the trial sequence. Interactive behaviors evoked during trial $\# 26$ of the communicative task in 4 different participant pairs. Three successful pairs showed different communicative behaviors, illustrating how different conversational contexts may evoke different communicative behaviors with the same meaning. For instance, subjectively interpreted, the Communicator of pair \#6 briefly pauses on the target location and then uses an "exit-point" strategy to indicate orientation, leaving that grid location along the direction to which the triangular token needs to point (Movie 3A) Communicator \#18 uses an "entry- and exit-point strategy", making two additional rotations at the target location to emphasize the need for the Addressee to rotate (Movie 3B). Communicator \#21 moves to the target location and rotates as many times as the Addressee has to rotate (Movie 3C). The interpretation of those behaviors is by no means trivial. For instance, in participant pair \#2 (Movie 3D), the Communicator makes a similar communicative behavior (two rotations at the target location) as the Communicator of pair \#18 (see Movie 3B), but it is interpreted differently by the respective Addressees. Argubly, Addressee $\# 2$ may have inferred from the Communcato actions that she needed to rotate twice, similar to the strategy used by pair \#21.

Supplemental Movie 4. Interactive behaviors evoked during trial \#30, \#32, \#46, and \#50 of the communicative task by the same participant pair (pair \#21). A communicative behavior can have different meanings in different trials, depending on the current conversational context of a pair. For instance, in trial \#30 the Communicator uses an "exit-point" strategy to indicate the orientation of the Addressee's triangular token, leaving the relevan grid location along the direction where the triangular token needs to point (Movie 4A). In trial \#32 (and onwards), the same player has started to use a "wiggle" strategy to indicate the target orientation of the triangle (Movie 4B). In trial \#46, the same player is presented (for the first time) with a goal configuration involving a triangle that points 'outwards'. In this trial, the wiggle is absent (Movie 4C). This absence is successfully interpreted by her Addressee as indicating an unusual orientation of the triangle. The success of this communicative interaction is even more remarkable given that in trial \#30 the Communicator produced a similar behavior to mean a different goa configuration. In this pair of participants, the absence of a wiggle as a mark for an outward pointing triangle is used in a few more trials (e.g. trial \#50, Movie 4D), until a different strategy is selected in later trials (not shown).
Supplemental Movie 5. Interactive behaviors evoked during trial \#9, \#11, and \#17 of the communicative task by the same pair of participants (pair \#9). A particular problem type can induce different communicative behaviors in different trials, depending on the current conversational context of a pair. For instance, in trial \#9 both participants' tokens are triangular, and the Communicator tries to convey to the Addressee her goal configuration by matching it with his own token (Movie 5A). This strategy, however, does not apply to trial \#11, where each player controls a differently shaped the Communicator chooses to wiggle to indicate the orientation of the triangle, and the meaning of this behavior is understood by the Addressee (Movie 5B). This shared symbol is also used in trial \#17 (Movie 5C), despite the fact that the problem presented in this trial is similar to the problem of trial \#9, and that in trial \#9 a different communicative behavior was used (see Movie 5A). token, forcing them to negotiate a different strategy. In this case

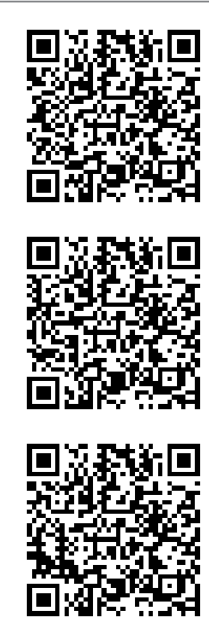

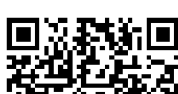

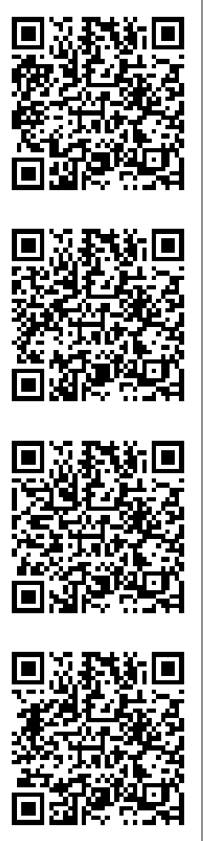




\section{Appendix B}

\section{A neurophysiological account of state- dependent processing during human communication}

Broadband shifts - the brain "at work". Brown Bag Meeting (2011), Donders Centre for Cognitive 


\section{Abstract}

A remarkable feature of the brain, and of the cerebral cortex in particular, is the ability to associate aspects of perceived experience with an internal representation of the world and make predictions about the future. How these complex cognitive processes are rapidly brought about by the intricate network of neurons that composes the human brain remains a mystery. The neural signal recorded by electrodes placed on the cortex or scalp is very rich, and in the past decades a large body of literature has been devoted to analyzing averaged evoked responses in the temporal domain, or focused more on rhythmic patterns observed in the spectral domain. However, increasing evidence suggest that the neural signal might contain highly relevant information beyond the evoked or rhythmic features. As I will outline in the present chapter appendix, a spatially resolved, non-oscillatory, broadband signal can be observed in the electrophysiological signal originating in the cerebral cortex, and it is in fact likely to be a macroscale correlate of population firing rate. This finding is based on simulation of neural activity based on a 'leaky integrate-and-fire' neuron model. Furthermore, in this appendix I will hypothesize about a relationship between the underlying mechanism of the broadband phenomenon and the phase of low-frequency rhythm, the blood-oxygenation-level-dependent (BOLD) signal, and higher cognitive functions (e.g. human communication)

\section{The noisy brain}

"Noise" typically is considered undesired as it interferes with the quality of a signal, but disregarding it completely without trying to understand the observed noise, or at least quantifying it, might also mean disregarding potentially relevant information. Since the discovery of the well-known alpha-brain-waves by Hans Berger in 1924, cognitive neuroscientists have had a strong focus on brain signal components within a special timescale, i.e. rhythmic activity. But what about non-oscillatory activity, i.e. the broadband signal we typically observe in the power spectral density spectrum computed from our brain signals? It has no special timescale and in the power spectrum it ends up 'everywhere' with a typical 1 over $f$ falloff with increasing frequency $f$ (see Figure 1A). Could it contain relevant information?

\section{Power-law scaling}

Many phenomena other than the brain signal power spectral density follow a power-law, such as the sizes of earthquakes, craters on the moon, solar flares, foraging patterns of various species, frequencies of words used in most languages, and frequencies of family names. The power-law, see equation below, refers to the $1 / f$ falloff of power spectral density $P$ with increasing frequency $f$.

$$
P \sim A \cdot f^{-x}
$$

where the coefficient $A$ determines the amplitude of the entire power spectral density and the scaling index $x$ determines the scale. Crucially in 'broadband shifts', the shape is preserved but the amplitude is not. Viewed in the frequency domain, the (temporal) power spectrum of arrhythmic brain activity roughly follows a straight line when plotted in coordinates of log power versus log frequency (see Figure 1B).

Shifts of energy in the entire power spectral density spectrum are referred to as broadband shift. Importantly, these broadband shifts reflect the changes in the amplitude of all frequencies, and not the exponent (the scaling index, e.g. the black line in Figure 1B). This latter feature of the brain signal is potentially equally interesting. Namely, the power-law exponent has been found to differ between brain networks and to correlate with fMRI signal variance and brain glucose metabolism (He, 2011; He, Zempel, Snyder, \& Raichle, 2010). But the scope of present investigation is restricted to changes in amplitude of the entire spectrum, i.e. broadband spectral changes, as these could represent a (macroscale) correlate of local neuron population activity (Miller, 2010). 


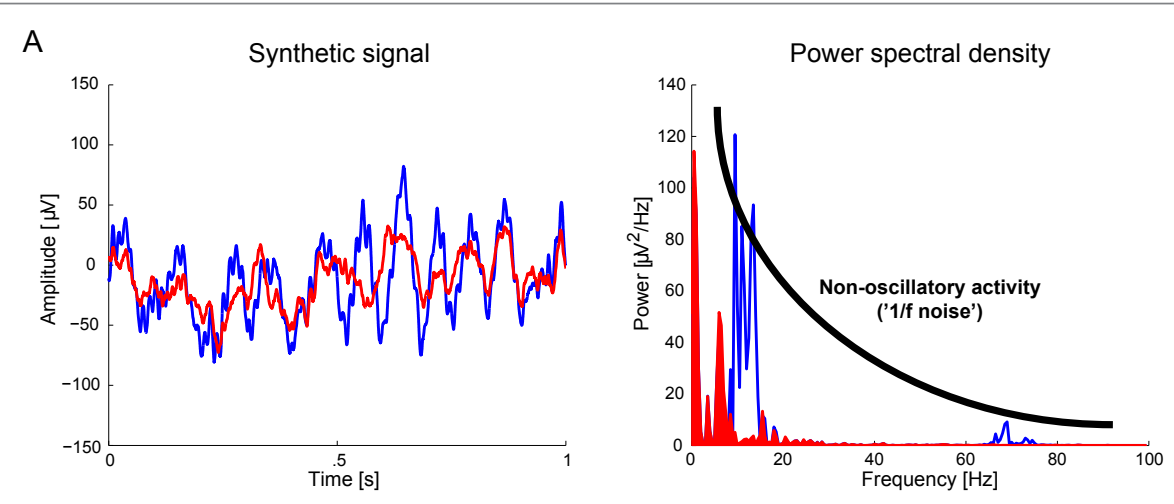

B
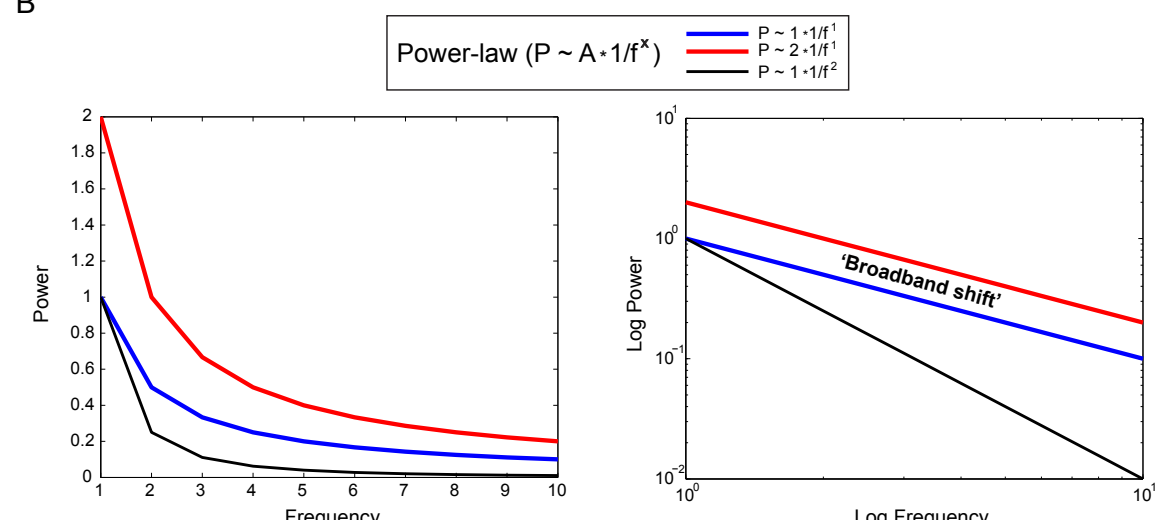

Figure 1. 'Broadband shift'. A) In the left panel, a one second synthetic signal consisting of an alpha-rhythm $(\sim 10 \mathrm{~Hz})$, a gamma rhythm $(\sim 70 \mathrm{~Hz})$, and nonoscillatory activity is shown in blue. The latter component is also shown in red in the same figure and, when spectrally resolved (right panel), is characterized by a $1 / \mathrm{f}$ falloff with increasing frequency; hence ' $1 / \mathrm{f}$ noise'. B) Broadband shifts refer to the change in amplitude A of the entire power spectrum (i.e. compare blue and red line in the left panel). When plotted in coordinates of log power versus log frequency, a frequency-consistent shift in amplitudes of the straight lines describing the power spectra can be observed. Note that in reality such a shift may be obscured by other processes (e.g. oscillatory activity) that may be of greater magnitude in the local field potential and of opposite direction.
In the remainder of this appendix, I will first describe where and when they have been found, and what is driving them. Second, I will generate broadband shifts, using a simulation involving a 'leaky-integrate-and-fire' neuron model. It will become apparent that the $1 / f$ shape in the power spectrum is likely related to synapse and membrane parameters ( $x$ in the power-law formula); the amplitude ( $A$ in the power-law formula) is related to spike firing rates. Third, I will briefly speculate and present my thoughts on links between broadband shifts and lowfrequency phase, the BOLD signal, and higher cognitive functions. This last point in particular may be of interest for the cognitive operations occurring in human communication, as we have seen in the present thesis chapter (chapter 3.1).

\section{Empirical findings}

Several studies have already shown the functional and behavioral relevance of broadband spectral changes. For example, in an electrocorticography (ECoG) study involving repetitive, self-paced finger movements (Miller, denNijs, et al. 2007; Miller, Leuthardt, et al., 2007; Miller, et al., 2009), an average shift in broadband spectral power was found in the signals of electrodes overlaying motor cortex when subjects started to make those movements as compared to rest. Power-law fits demonstrated a scaling index $x$ of 4 for frequencies $>80 \mathrm{~Hz}$ and 2 for $<80 \mathrm{~Hz}$, consistent over subjects. Furthermore, there was a 'knee', i.e. a bump, around this frequency.

Another study (Ray \& Maunsell, 2011) highlights the functional difference between the gamma-rhythm and what the authors call 'high-gamma activity' (between 50 and $150 \mathrm{~Hz}$ ). When a larger stimulus was shown, gamma rhythm power at $50 \mathrm{~Hz}$ increased simultaneously in macaque primary visual cortex, but high-gamma activity favored the smaller stimulus. Furthermore, the authors found a relation between this high-gamma activity and neuronal firing rates. In fact, this correlation had a different, negative sign for the gamma rhythm. These results distinguish broadband high-gamma activity from gamma oscillations as an index of neural firing near the microelectrode. And it suggests that highgamma activity is not the same type of process as gamma oscillations, simply operating at a higher or broader frequency. Instead, it seems to be related to spiking activity and these results therefore highlight the importance of making a careful dissociation between gamma rhythms and broadband/high-gamma activity.

Here I would like to postulate that this so called high-gamma activity might actually be a reflection of a broadband shift (a shift in power at each frequency) of which the low-frequency portion may become obscured at lower frequencies 
by coincident changes in rhythmic phenomena. This rationale is reinforced by another study in which human subjects were recorded intracranially when performing a virtual navigation task (Manning, et al., 2009). The researchers found that the overall power in the local field potential (LFP) powerspectrum changed over time. Then they found that the changes in neuronal firing rate were concurrent with those changes in broadband LFP power. Manning and colleagues thus demonstrated that broadband spectral change in the electric potential is correlated with neuronal action potential firing rate.

Recent measures of activity in cell assemblies of zebrafish olfactory bulb underscore the functional relevance of the temporal characteristics of those firing rates. Namely, it was found that subsets of output neurons (mitral cells) engage in synchronized oscillations during odour responses, but information about odour identity was contained mostly in the non-oscillatory firing rate pattern (Blumhagen et al., 2011).

Finally, it is worth mentioning here that broadband shifts have also been found in a recent electroencephalography (EEG) study (Onton \& Makeig, 2009). EEG is typically measured extracranially and may capture brain signals that are smeared by the skull, as compared to ECoG. It typically has a lower signal-tonoise ratio and may therefore require more advanced techniques (i.e. involving spatial filtering) to reconstruct small effects on the signal originating from the brain. Onton and her colleague used a non event-related paradigm without external stimulation. They found broadband shifts originating from sources in anterior temporal lobe with the imaging of emotional states. They further illustrated that there are more sources that can produce broadband spectral changes and that typically mix with the EEG signal, such as eye, head, and jaw muscles, emphasizing the relevance of the advanced signal analysis techniques that were used.

\section{Simulation}

The empirical findings mentioned above support the idea that a relation exists between broadband LFP power and neuronal firing rate. We will perform a relatively simply simulation to provide us insight into this relation. In this simulation, we will simulate the effect of neuronal firing on the local field potential of a pyramidal cell in grey matter. Our ECoG/EEG signal is best seen as the spatial average over many of these LFPs. Beneath one electrode, there are many neurons (see Figure 2A), each having many synapses where they receive inputs. For our simulation, we will focus on one pyramidal neuron of such a population. We will generate broadband shifts using three steps:
1) During active computation, the population of pyramidal neurons engages in a complex, Poisson-distributed (noise-like) pattern of mutual excitation (indicated with label ' 1 ' in Figure 2B).

2) Each arriving action potential from a presynaptic neuron induces a post-synaptic current. Its temporal shape has an extremely fast rise and an exponential decay (label '2' in Figure 2B).

3) While the temporally integrated postsynaptic charge (bolus) diffuses toward the soma, it loses current across the dendritic membrane (label ' 3 ' in Figure 2B).

The combination of these three processes is thought to be the source of macroscale potentials as picked by an ECoG/EEG electrode. The last two steps can be seen as the origin of the $1 / f$ shape in the power spectrum. The first step determines the amplitude of the entire power spectrum.

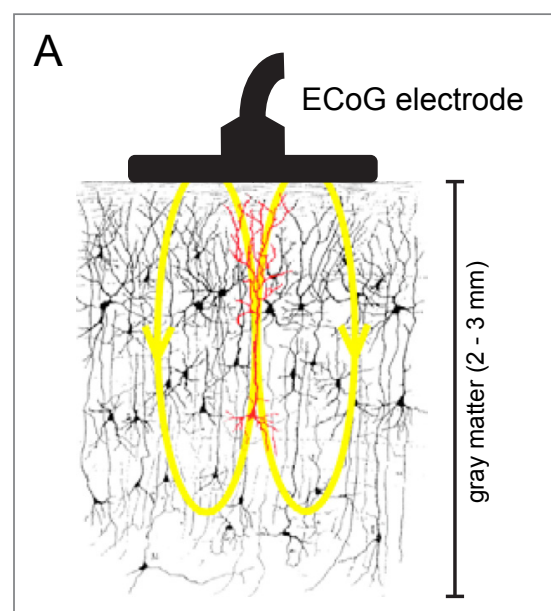

B

Figure 2. Situation and steps considered in the simulation of local neural activity. A) Beneath one electrode, there are many neurons. B) A single neuron receives roughly 6000 presynaptic inputs at its synapses (indicated in blue). 1) During active computation, the population of pyramidal neurons engages in a complex (noise-like) pattern of mutual excitation. 2) Each arriving action potential from a presynaptic neuron induces a post-synaptic current. Its temporal shape has an extremely fast rise and an exponential decay. 3) The input from all synapses is combined, and charge accumulates over time and is lost, ohmically, through the dendritic membrane. Figure adapted from (Miller, et al., 2009). 
It is worth emphasizing here that, unlike axonal action potentials, dendritic spikes do not propagate reliably over long distances in dendrites. If dendritic spikes fail to entirely propagate to the soma, this results in subthreshold somatic depolarization, and no action potential is triggered in the axon. Although no downstream action potential is triggered, changes in the transmembrane potential are occurring. The time dependence of transmembrane potentials are captured by the LFP, and likely by the macroscale potential as well (Okun, Naim, \& Lampl, 2010). That is, the spatially weighted average of the synaptic transmembrane currents.

In the simulation, actions potentials with poisson-distributed arrival times arrive at a neighboring neuron (i.e. step 1). I here consider input from 6000 presynaptic neurons, each firing 10 spikes per second, for a total time of 2 minutes. These have different strengths (related to the distance to the soma) and directions of effect (inhibitory/excitatory) on the (hypothetical) transmembrane potential. The sum of this presynaptic input is a very noisy signal (e.g. orange line in left panel of Figure 3A). In fact it can be characterized as white noise; each frequency is equally represented (see orange line in right panel of Figure 3A). Second, each arriving action potential triggers release of a neurotransmitter and postsynaptic current influx. We simulate this by performing a convolution of the signal with a function that has a sharp rise and exponential decay. This produces a $1 / f^{2}$ form following a kink (the 'knee') at a frequency determined by the decay time at the synapse (see orange line in right panel of Figure 3B). Third, we simulate the integration of charge from synaptic inputs of 6000 synapses over time, counteracted by Ohmic leakage, i.e. loss across the dendritic membrane. This operation causes the trans-membrane difference in charge concentration to be perturbed, acting as another low-pass filter (see orange line in right panel of Figure 3C)

We could repeat the exact same steps as outlined above and create broadband shifts by simply change one parameter: the input spike rate in step 1 . Compare the orange (on average 10 spikes/s) and purple lines (40 spikes/s) throughout the panels of Figure 3 for the effect of simulating not 10 but 40 spikes per second, i.e. more presynaptic activity. In summary, the broadband amplitude modulation is caused by a change in input spike rate, or mean population firing rate. The $1 / f$ falloff with frequency results from the shape of synaptic current decay and the effect of temporal (and spatial) integration in the dendritic arbor (steps 2 and 3 respectively).

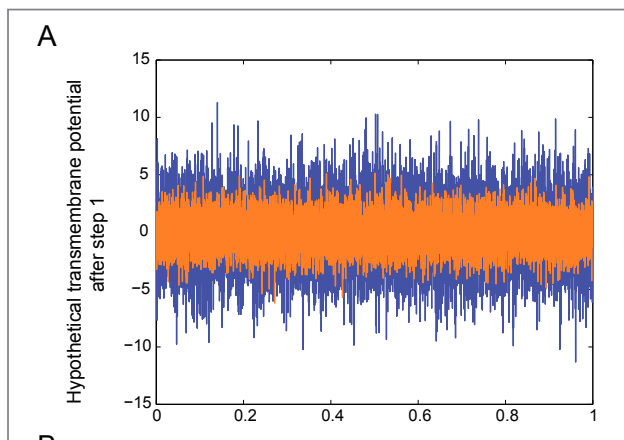

$B$
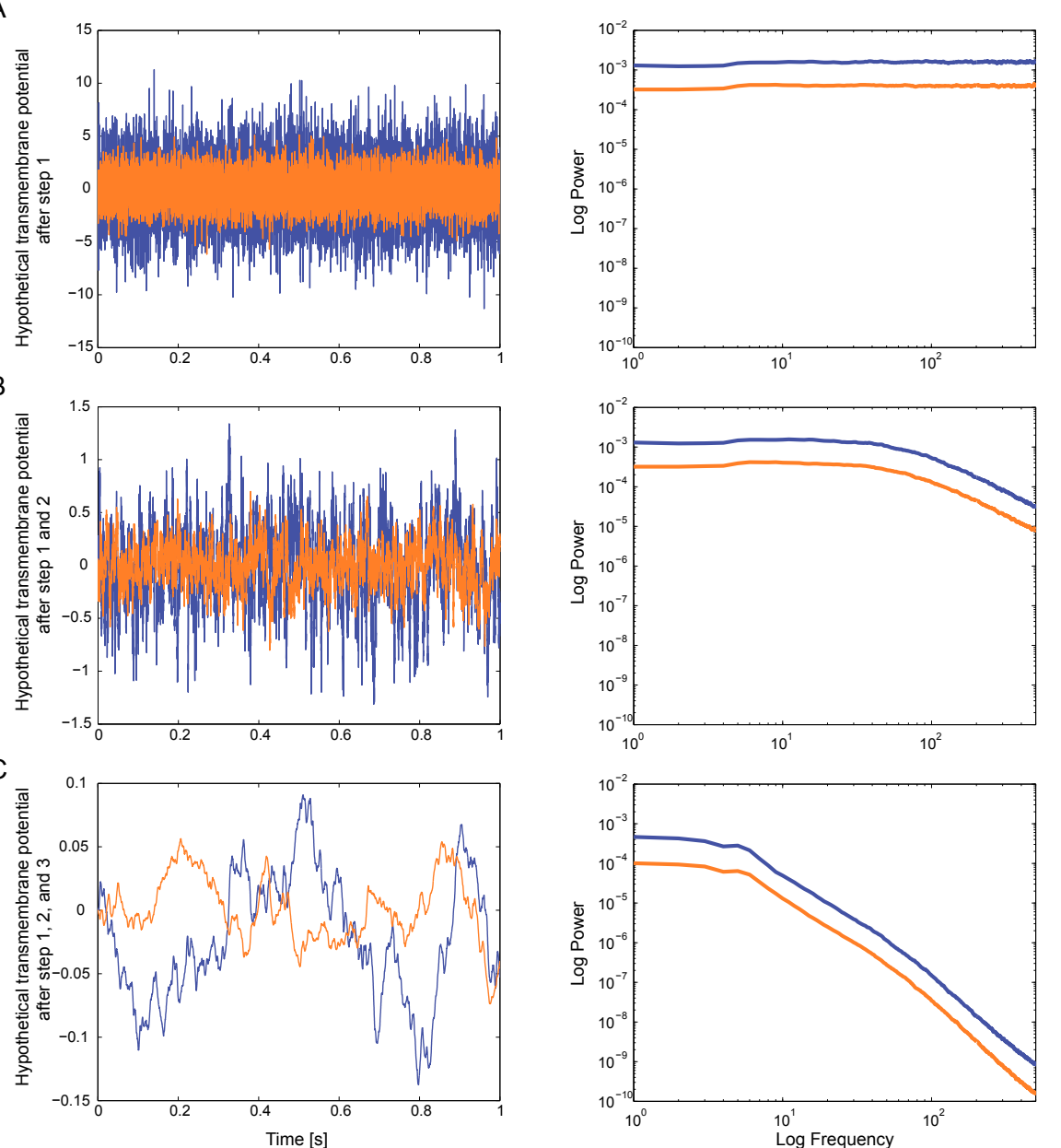

${ }_{\text {Log Frequency }}^{10^{1}}$

Figure 3. Simulation of broadband shifts in the local field potential by changing the input spike firing rate. A) The sum of 6000 presynaptic input is a very noise signal, i.e. white noise. Note that the change in spike firing rate (on average 10 vs. 40 spikes/s) already determines the broadband spectral change (right panel). B) Each arriving action potential triggers release of a neurotransmitter and postsynaptic current influx. This produces a $1 / f^{2}$ form following a kink (the 'knee') at a frequency determined by the decay time at the synapse. C) Then the charge from synaptic inputs of 6000 synapses is integrated over time, counteracted by Ohmic leakage, i.e. loss across the dendritic membrane. This operation causes the trans-membrane difference in charge concentration to be further perturbed, acting as another lowpass filter on the membrane potential time series. 


\section{Coupling with low-frequency phase}

A recent intriguing finding provides more detail on the functional specificity of broadband shifts. Miller and colleagues (Miller et al., 2010) found that the broadband amplitude was inversely correlated with amplitude of the alpha rhythm. A more detailed analysis demonstrated that the broadband amplitude was modulated by the phase of the rhythm. The authors speculate that the alpha rhythm reflects synchronized input to a cortical population of neurons, i.e. a synchronizing neuron' (rhythm generator) is synchronously driving 'inhibitory neurons' that on their turn deliver, roughly simultaneously, input near the soma of separate pyramidal neurons. They further reasoned that whether these synchronized inputs are excitatory or inhibitory on a single-neuron scale, the cortico-cortical inputs between pyramidal neurons will need to be stochastically resonant with the synchronized input to induce a downstream action potential (on a population scale). In other words, weak but synchronous input keeps the entire pyramidal population in a 'dynamically suppressed' state, thus characterized by smaller broadband amplitude in the macroscale potential.

\section{BOLD signal}

Hermes and colleagues (Hermes et al., 2012) recorded the hemodynamic response and ECoG of subjects making simple finger movements (visually cued thumb/finger flexion at the rate of $2 \mathrm{~Hz}$, for 30 seconds). They found that the blood oxygen level dependent signal (BOLD signal) was related to both low frequency (inversely and spatially distributed) and high frequency power modulations (positively and spatially focal). Interestingly, although the study setup did not allow measurement higher than $100 \mathrm{~Hz}$, that high frequency band power modulation might be reflection of a broadband shift with the lowfrequency portion obscured by low-frequency rhythms having their effects in opposite direction. In line with the above sections, the high frequency signal power may thus be related to local, cortico-cortical neuronal activity whereas the low frequency rhythms $(<30 \mathrm{~Hz})$ can be thought to reflect an aspect of cortical-subcortical interaction. Building further on the bridge between broadband shifts and the BOLD signal, the BOLD signal has been found related to the LFP (Logothetis, Pauls, Augath, Trinath, \& Oeltermann, 2001), and thus, to presynaptic spiking rate as detailed earlier in this appendix. Accordingly, I would like to postulate that a part of the BOLD signal may be the hemodynamic equivalent of electrophysiological broadband shifts. One way to investigate this may involve the use of a task that is known to separate the different brain signal phenomena of gamma rhythm and broadband shifts (e.g. the task used in (Ray \& Maunsell, 2011)) while simultaneously recording brain activity with EEG and fMRI.

\section{Higher cognitive functions}

As mentioned earlier, dendritic spikes do not propagate reliably over long distances in dendrites. If dendritic spikes fail to entirely propagate to the soma, this results in subthreshold somatic depolarization (but with consequences for the local field potential of that neuron), and no action potential is triggered in the axon. This feature of a pyramidal neuron's dendrite has led neuroscientists (Behabadi, et al., 2012; Jarsky, et al., 2005; Larkum, 2012) to suggest that it opens the way for two excitatory input streams contacting the same dendrite to interact in an asymmetric non-linear way that depends on their absolute and relative locations. For instance, enhanced local cortico-cortical interactions lead to enhanced forward propagation (by increasing the neuron's excitability) of distal synaptic inputs (Jarsky, et al., 2005). This previously unknown form of spatial analog computation has been suggested to be ideally suited to support interactions between classical "driver" inputs that give rise to their basic response properties, and "contextual" inputs that nonlinearly modulate those responses (Behabadi, et al., 2012).

Building on this notion, an increase in noise-like cortico-cortical interaction, electrophysiologically represented by a broadband shift at the macroscale potential, may be a suitable mechanism for holding the neural population near its excitability threshold in a state-dependent manner. It is an intriguing idea that this mechanism could already operate before the occurrence of a sensory event itself, holding online high-level representations, to be integrated with expected low-level sensory input. In the present thesis chapter (chapter 3.1), we have observed broadband shifts in cortical areas known to be necessary for processing conceptual knowledge and mental models of other agents (Lambon Ralph, et al., 2010; Milne \& Grafman, 2001; Sabbagh, 1999), i.e. right temporal lobe and ventromedial prefrontal cortex, during human communicative interactions. Interestingly, these broadband shifts emerged already before the occurrence of a specific communicative problem, raising the question whether we might have captured the putative mechanism described above in operation. Currently, this remains an open question, but the empirical findings and simulation work presented here support the idea that this noise-like phenomenon is related to neural processes, and that therefore it is worth further investigation. 


\section{Cerebral coherence}

between communicators marks the emergence of meaning

Stolk A, Noordzij ML, Verhagen L, Volman I, Schoffelen JM, Oostenveld R, Hagoort P, Toni I 


\section{Introduction}

Imagine you are taking your friend to the movies to cheer her up after she recently broke-up with Tom. Suddenly, you recognize Tom's bicycle parked near the cinema. By virtue of a pointing movement directing her attention to his bike, you have managed to silently ask her whether she wants to go somewhere else instead (Tomasello, 2008). When two friends of Tom see the same bike minutes later, the exact same pointing movement is made again, but with a radically different meaning. Selecting and understanding the meaning of communicative signals relies on knowledge and beliefs knowingly shared across the participants of the communicative act. This "common ground" is specific to the context and participants of the interaction (Clark, 1996), being continuously negotiated between interlocutors (Brennan, et al., 2010; Levinson, 2006). It is widely accepted that common ground is crucial for disambiguating the meaning of the symbols used during a dialogue (Clark, 1996), but it is unknown how common ground is cerebrally implemented (Schilbach, et al., 2013).

\section{Abstract}

Commonground, orknowledge knowinglyshared among communicators, provides a cognitive context necessary to disambiguate the meaning of the symbols used in human referential communication. Current accounts of dialogue suggest that interlocutors generate common ground by predicting individual sensory tokens used during a communicative interaction. Alternatively, communicators could create common ground by reasoning over conceptual features shared across multiple instances of transient sensory tokens. Both hypotheses predict neural dynamics coherent across communicators, but at different frequencies. Here we use fMRI to simultaneously record brain activation in pairs of participants building common ground over multiple communicative interactions. As common ground emerged within a pair of interlocutors, activity in the right superior temporal gyrus also increased, during both production and comprehension of a communicative action. This increased activity was coherent across participants of a pair ove periods spanning multiple communicative interactions, well above the occurrence of individual sensory tokens. This slow-frequency withinpair coherency was considerably stronger than that measured in pairs of communicators not sharing a conversational context. These findings indicate that establishing common ground relies on shared, pair-specific reasoning about conceptual features of an ongoing interaction, rather than about the sensory tokens of the interaction.

We reasoned that interlocutors generate common ground by predicting individua sensory tokens used during a communicative exchange (Hasson, et al., 2012; Pickering \& Garrod, 2013); or by reasoning over conceptual features shared across multiple instances of transient sensory tokens (Brennan, et al., 2010; Levinson, 2006). Both possibilities predict neural dynamics coherent across communicators, with the former at frequencies driven by the occurrence of individual tokens and the latter at much lower frequencies driven by adjustments of more abstract conceptual representations. This study investigates the cerebra mechanisms supporting the emergence of common ground by manipulating its dynamics, in the context of an experimentally controlled communicative task ((de Ruiter, et al., 2010), Figure 1A). The experimental setting precludes the use of pre-established communicative conventions (e.g. a common idiom, body emblems, facial expressions), thereby focusing communicators towards the generation of mutually negotiated shared meanings (Stolk, Verhagen, et al., 2013). Furthermore, by monitoring both elements of a communicative pair, simultaneously, we could capture pair-specific neural dynamics reflecting the shared creation of common ground, over and above neural effects evoked by each communicative token (Brennan, et al., 2010; Levinson, 2006). 


\section{Materials and Methods}

\section{Participants}

Fifty-four male participants (assigned to twenty-seven communicative pairs, ages 18-27 years), were recruited to take part in this study. They were screened for a history of psychiatric and neurological problems and had normal or corrected-to-normal vision. Participants gave written informed consent according to institutional guidelines of the local ethics committee (CMO region Arnhem-Nijmegen, The Netherlands) and were either offered a financial payment or given credits towards completing a course requirement.

Task

At interaction onset, each player is assigned a token (epoch 1: token assignment, Figure 1A). Then the Communicator (and the Communicator only) is shown the target configuration of that interaction (epoch 2: planning). The target configuration contains the tokens of the Communicator and the Addressee, at the grid locations and orientations they should have at the end of the interaction. The Communicator knows that the Addressee does not see the target configuration, and that he cannot move the Addressee's token. Therefore, the Communicator needs to communicate to the Addressee the location and orientation that her token should have at the end of the interaction. To comply with the task requirements, the Communicator also needs to ensure that at the end of his turn his token (in blue) is at the location and orientation specified by the target configuration. In this game, the only means available to the Communicator for communicating with the Addressee is by moving his own token around the grid, namely horizontal translations, vertical translations, or clockwise rotations. Both Communicator and Addressee also know that the Communicator has unlimited time available for planning his moves, but only five seconds for moving his token on the grid. The Communicator signals his readiness to move by pressing the start/stop button. At this point, the target configuration disappears, the Communicator's token appears in the center of the grid, and he can start moving his token (epoch 3: movement). After five seconds, or earlier if the Communicator hits the start/stop button again, the Communicator's token cannot move further and the Addressee's token appears in the center of the grid. This event indicates that the Addressee has acquired control over her token. The Addressee has unlimited time to infer the target location and orientation of her token on the basis of the observed movements of the Communicator (epoch 4: planning). After the Addressee presses the start/stop button, she has five seconds to move her token (epoch 5: movement) Finally, after five seconds, or earlier if the Addressee hits the start/stop button again, the same feedback is presented to both players in the form of a green tick or red cross (positive or negative feedback, respectively; epoch 6: feedback). The feedback indicates whether the participants had matched the location and orientation of their tokens with those of the target configuration.

It should be noted that the Addressee cannot solve the communicative task by reproducing the movements of the Communicator's token. Rather, the Addressee needs to disambiguate communicative and instrumental components of the Communicator's movements, and find some relationship between the Communicator's movements and their meaning. Second, there are no a prior correct solutions to the communicative task, nor a limited set of options from which the Communicator could choose.

\section{Experimental procedures}

An experiment totaled two parts; one training session outside the scanners, and one recording session in which functional brain images were acquired on both participants of the pair simultaneously. Prior to the training session, the participants were individually trained with using the hand-held controller (40 interactions of token configuration matching) and jointly familiarized with the general procedures and events of the interactive task (10 interactions with no communicative demands).

The training session allowed participants to get familiarized with the communicative aspect of the task. In fact, unbeknownst to the participants themselves, within this session the participants were meant to establish common ground on a set of communicative problems. The training session was completed only when the participants had successfully accomplished a significant amount of subsequent interactions. They completed at least 25 interactions, and if they had a negative outcome on one of the last ten interactions, they had to complete ten additional interactions until they jointly solved all those ten interactions correctly.

The fMRI session consisted of 84 interactions. Half of these interactions consisted of the problems previously faced by the participant pair within the training session, now labeled as 'Known' interactions. These interactions were intermixed with 'Novel' interactions, i.e. target configurations that were not presented to the pair before. Not more than either three Known or three Nove interactions were presented sequentially. There were four different problem types involving different token shape and orientation combinations (to build the target configurations) used within the Known and Novel interactions. For the Known interactions these were triangle-rectangle, rectangle-circle, 
triangle-circle, and triangle*-circle for the Communicator-Addressee token pair (*triangle pointing outwards the game board). For the Novel interactions these were rectangle-triangle, circle-rectangle, circle-triangle (e.g. Figure 1A), and circle-triangle*. As compared to the Known interactions, in these interactions the Communicator's token had fewer orientations than the Addressee's token. This deliberate mismatch between the geometrical characteristics of the tokens of Communicators and Addressees increased the communicative task difficulty. For instance, when the Communicator's token was a circle and the Addressee's token was a triangle (e.g. Figure 1A), then the Communicator needed to find a new way to indicate to the Addressee the orientation of her token, since rotations of the circle token were not visible. A further level of difficulty could be introduced by using a triangular token pointing outward the grid (denoted by the asterisk symbol) as the Addressee's target configuration, the Communicator's token being a circle. The presentation order of problem types was intermixed for both the Known and Novel interactions. When a pair jointly solved four Novel interactions of the same problem type consecutively, it was assumed that the pair had established a communicative rule for that problem type and that specific problem type was substituted by other types for the remainder of the experiment. The rationale of this intervention was to drive participants to continuously create new communicative behaviors, rather than exploiting already established communicative conventions during the Novel interactions.

\section{fMRI image acquisition and analysis}

Communicators were scanned using a 3 Tesla Trio scanner (Siemens, Erlangen, Germany). BOLD sensitive functional images were acquired using a single shot gradient echo planar imaging (EPI) sequence (TR/TE 2.60s/40ms, 34 transversal slices, interleaved acquisition, voxel size $3.5 \times 3.5 \times 3.5 \mathrm{~mm}^{3}$ ). Structural images were acquired using a MP-RAGE sequence (TR/TE $2.30 \mathrm{~s} / 3.9 \mathrm{~ms}$, voxel size 1 $\mathrm{x} 1 \mathrm{x} 1 \mathrm{~mm}^{3}$ ). Addressees were functionally scanned using a 1.5 Tesla Sonata scanner (Siemens, Erlangen, Germany) and a single shot gradient EPI sequence (TR/TE $2.70 \mathrm{~s} / 40 \mathrm{~ms}, 34$ transversal slices, ascending acquisition, voxel size 3.5 x $3.5 \times 3.5 \mathrm{~mm}^{3}$ ). Structural images were acquired using a MP-RAGE sequence (TR/TE $2.25 \mathrm{~s} / 3.68 \mathrm{~ms}$, voxel size $1 \times 1 \times 1 \mathrm{~mm}^{3}$ ).

The images were preprocessed and statistically analyzed using SPM8 (Statistical Parametric Mapping; www.fil.ion.ucl.ac.uk/spm). Preprocessing of the functional scans included brain extraction (Smith, 2002), spatial realignment (rigid body transformations using sinc interpolation algorithm), slice-time correction, coregistration (of functional and anatomical images, after prior coregistration of both image types to an MNI template), reslicing (1.5 x $1.5 \mathrm{x}$
$1.5 \mathrm{~mm}$ ), spatial normalization (to MNI space), and spatial smoothing (isotropic $8 \mathrm{~mm}$ FWHM Gaussian kernel). Each anatomical image was segmented into three different tissue compartments (grey matter, white matter, cerebral spinal fluid). Mean signals in the latter two compartments and head movement related parameters (translations and rotations to the mean as obtained during the spatial realignment, and their first derivatives) were entered as regressors in all first level fMRI analyses. Furthermore, the data was high-pass filtered (cut-off $128 \mathrm{~s}$ ), and temporal autocorrelation was modeled as an AR(1) process.

The seven epochs shown in Figure 1A were considered for the first level fMRI analyses of both Communicators and Addressees. The feedback event (epoch 6, Figure 1A) was modeled separately based on the positive or negative outcome of a interaction. Each epoch time series was convolved with a canonical hemodynamic response function and used as a regressor in the SPM multiple regression analysis. Following our hypothesized difference in neural activity, we modeled specific epochs separately for the known and novel interactions, i.e. pertaining to the planning periods of Communicators and the observation periods of Addressees (both with variable durations). We additionally considered the time modulatory effects of one parameter on those specific epochs; the behaviorally obtained change in joint performance on the novel interactions (green curve in Figure 1D, see Supporting Information). This curve roughly followed a logarithmic over the course of the experiment, and we modeled the parametric modulation of planning (Communicators) and observing epochs (Addressees) using the log transformed interaction number for the novel and known interactions separately. For visualization purposes of these time modulatory effects, we considered seven planning and observation regressors instead of one for the known and novel interactions. Each regressor encompassed six interactions in chronological experimental order (i.e. 'scanning time bin', see Figure 2).

Coherence analyses were performed using the FieldTrip toolbox for MEG/EEG analysis (Oostenveld, et al., 2011) and custom MATLAB code (MathWorks, Natick, MA, USA). Whole-experiment neural traces were extracted from regions of interest, synchronized to experiment start, band-pass filtered (0.0025 - 0.02 $\mathrm{Hz}$ frequency-domain brick-wall), and nuisance-corrected using mean signals of grey matter and cerebral spinal fluid compartments and head movement related parameters. The neural traces of Addressees were resampled to match the sample frequency of Communicators (TR of 2.70s as compared to 2.60s) For each participant, the neural time series was segmented into multiple consecutive overlapping windows of 400s. The windowed time series were tapered with a set of 3 orthogonal Slepian tapers prior to spectral estimation 
and calculation of the magnitude squared coherence. This resulted in a spectral smoothing of $\pm 0.005 \mathrm{~Hz}$

\section{Statistical model and inference}

Our main interest was the time-invariant and time-variant differences in planning (Communicators, epoch 2 in Figure 1A) and observation (Addressees, epoch 3) related neural activity between the known and novel interaction types. We therefore considered the contrasts Known vs. Novel, Novel vs. Known, and the time/interaction dependent changes in neural activities on the Known (as a control, joint performance did not change over time for this interaction type, see Figure 1D) and Novel interactions by testing the null hypothesis of no change. These contrasts were entered into a full factorial model with Role (Communicator, Addressee) as between-subjects variable, and Interaction type (Known, Novel) and Time (Invariant, Variant) as within-subjects variable, treating subjects as a random variable. Unequal variance between the conditions was assumed and the degrees of freedom were corrected for nonsphericity at each voxel. We report the results of a random effects analysis, with inferences drawn at the cluster level, corrected for multiple comparisons over the whole brain using family-wise error correction ( $\mathrm{p}<0.05$, (Friston, Holmes, Poline, Price, \& Frith, 1996)). We play on the strength of conjunction analyses to isolate neural effects overlapping between the two roles, Communicators and Addressees (Nichols, et al., 2005).

Interlocutor pair-specificity of neural signal coupling was tested by comparing coherences calculated on signals of participants with their communicative partners $\left(\mathrm{N}_{\text {pairs }}=27\right)$ with those calculated on signals of elements of different pairs (e.g. Communicator from pair \#1 with Addressee from pair \#2; N

$=702$; i.e. $27 \times 26$ ). The coherence measures were entered into a second-level random effects analysis correcting for multiple comparisons at the cluster level (Monte Carlo $p<0.05$; 10,000 randomizations across participant pairs, (Maris \& Oostenveld, 2007)).

\section{Results}

Twenty-seven pairs of participants were asked to jointly create a goal configuration of two geometrical tokens, using the movements of the tokens on a digital game board as the only available communicative channel. One member of a pair, the Communicator, knew the goal configuration, and he moved his token on the game board with his right hand to inform an Addressee where and how to position his token (Figure 1A, epochs 2 and 3). Details of this communicative task are described in the Materials and Methods. This task has proven effective in encouraging the generation of pair-specific communicative behaviors (Blokpoel, et al., 2012; de Ruiter, et al., 2010; Volman, et al., 2012) The same movements could be used by different pairs to negotiate different meanings, and the same meaning could be conveyed by different movements across different pairs. The same movement could even be used to convey different goal states by the same pair in different interactions, and vice versa (Stolk, Verhagen, et al., 2013). The latter observation emphasizes how in this game, a movement acquires meaning by virtue of the shared history of the communicative interactions within a given pair, rather than by virtue of its sensory attributes.

Cerebral activity of each pair was simultaneously monitored with BOLD-sensitive fMRI, while we experimentally manipulated common ground dynamics using two types of communicative problems (42 interactions each, intermixed such that no same type was presented more than 3 times sequentially). There were problems in which the pairs already had previously established common ground ('Known' interactions, with stable performance), and there were problems in which common ground yet had to be established ('Novel' interactions, with increasing performance). We first examined the main neural effect of having access to common ground on the selection (Communicators, epoch 2 in Figure 1A) and comprehension of communicative actions (Addressees, epoch 3). Given the large differences in the sensory inputs and motor outputs of those task epochs, overlapping neural activity should be related to abstract task features shared across the interlocutors. Despite overall differences in behaviora performances between the Known and Novel interactions (cf. performances in Figure 1B-D, and see Supporting Information), strikingly overlapping brain regions between Communicators and Addressees were activated more strongly during the Known than the Novel interactions, and vice versa (Figure S1). 

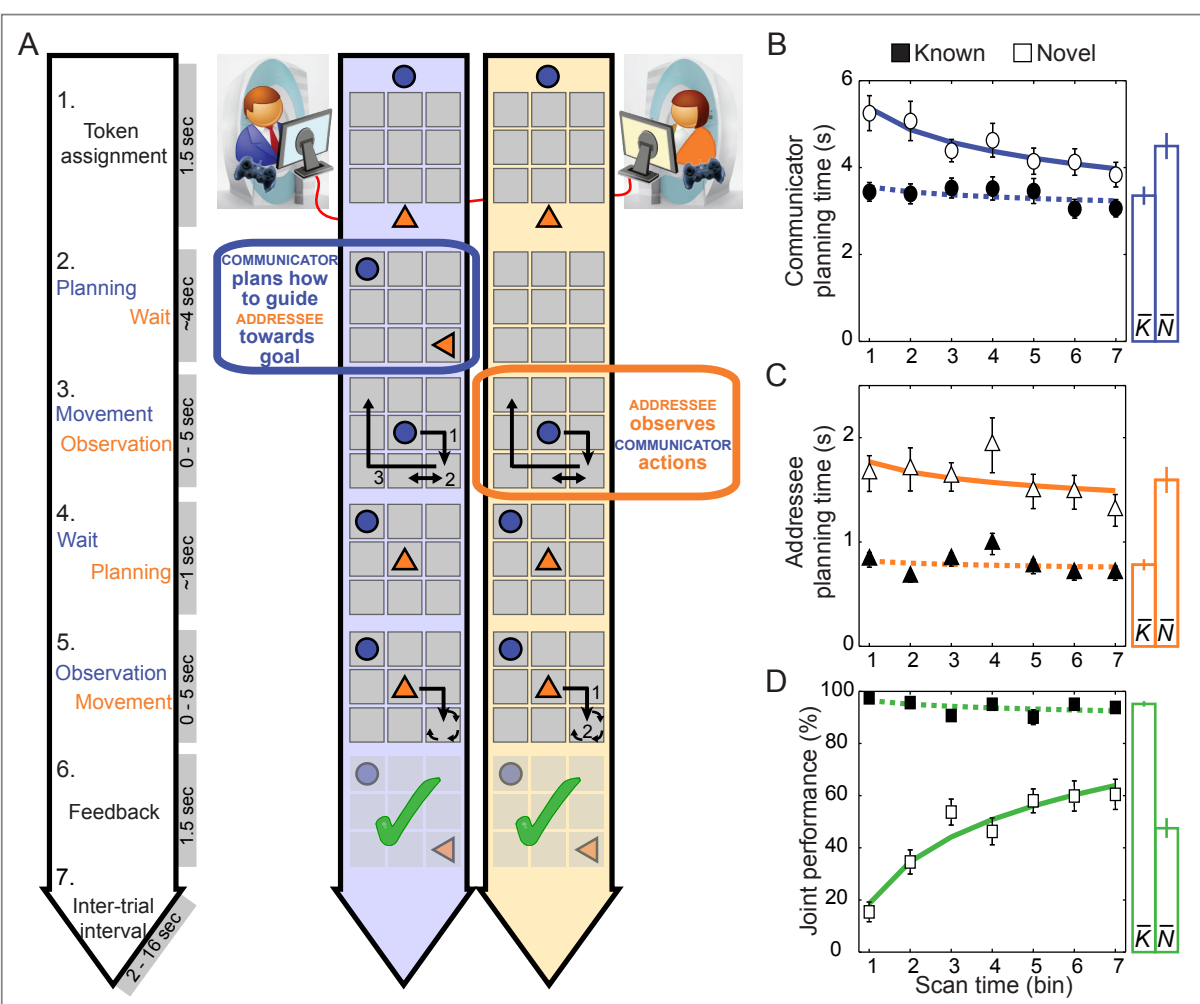

Figure 1. Communicative interaction. (A) The target configuration was shown to the Communicator only (Communicator epoch 2). To achieve that target configuration, the Communicator needed to convince the Addressee to move her token (in orange) to the desired target location and orientation. The Communicator could achieve this only by moving his token (in blue) across the digital grid, knowing that the Addressee will observe those movements (Addressee epoch 3) to decide where and how to move her token (Addressee epoch 5). Success of a communicative interaction relied on the Communicator designing an action that could be understood by the Addressee (during planning in epoch 2), and on the Addressee inferring the Communicator's intentions (during observation in epoch 3). (B and C) Planning times of Communicators and Addressees over scanning time (binned in blocks of 6 interactions) for interactions in which the pairs already had previously established common ground ('Known' interactions) and for interactions in which common ground yet had to be established ('Novel' interactions). (D) Joint performances of Communicator and Addressee pairs for the two interaction types. The change in joint performance on the Novel interactions roughly follows a logarithmic (green curve). Error bars indicate \pm 1 SEM.
Given the prediction that neural activity changes with the emergence of meaning within each pair, we proceeded to investigate the change in joint task performance on the Novel interactions over the course of the experiment. This change was approximated by a logarithmic function, $F(1,40)=51.6, p<<$ $0.001, \mathrm{R}^{2}{ }_{\mathrm{adj}}=.55$ (cf. the green curve in Figure 1D). Searching for changes in brain activity following the same temporal profile across both Communicators and Addressees highlighted a region with activity increasing over the course of the experiment during Novel interactions (Figure 2A). Namely, the right superior temporal gyrus (STG, Brodmann Area 48; peak activation in rolandic operculum, MNI $[63,-6,9])$, showed a significant increase in activity during Novel interactions (Figure 2B), approaching activity levels observed in the Known interactions by the end of the experiment (masks: Known $>$ Novel and Novel $_{\text {time }}>$ Known $_{\text {time }}$, cf. Figure S1 and Figure S2). Similarly to the joint performance of each interlocutor pair, activity in right STG thus incrementally builds up during the Novel interactions (blue and orange solid lines, see Figure 2B) but not during the Known interactions (dashed lines).
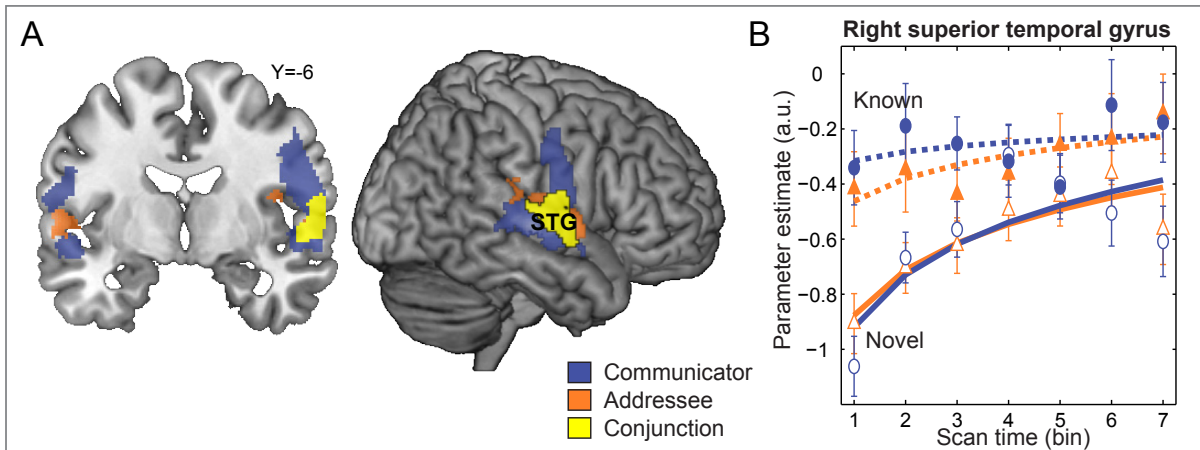

Figure 2. Acquisition of shared meaning. (A) Brain regions whose activity follows the same dynamics as joint communicative performance on the Novel interactions within each pair (cf. green curve in Figure 1D). The conjunction shows the commonalities in neural activities between the two roles (i.e. Communicators planning, epoch 2 in Figure 1A, Addressees observing, epoch 3). The statistical maps are masked (for presence of common ground and time-specificity on Novel interactions, cf. Figure S1 and S2) and thresholded at $p<0.05$, whole-brain FWEcorrected, see Table S1 for details. (B) Parameter estimates of activity evoked in right superior temporal gyrus (STG) over scanning time (binned in blocks of 6 interactions) for interactions in which the pairs already had previously established common ground (Known interactions - filled markers) and for interactions in which common ground yet had to be established (Novel interactions - empty markers). For planning (Communicators - blue markers) and observing communicative actions (Addressees - orange markers), the estimated signal is mean-baseline-subtracted. The data points are fitted with a logarithmic function. 
Next, we tested the prediction that the emergence of meaning during a communicative interaction is specific to the context and the participants of the interaction, using coherence analysis of the BOLD signal across pairs. This model-free analysis also allowed us to assess the spectro-temporal fingerprint of signal in right STG, investigating whether the increases in activity over the course of the experiment are caused by stronger evoked responses associated with stimulus material or by abstract task features of the communicative interactions. We observed strong within-pair coherence of the neural dynamics in this region at $0.01-0.04 \mathrm{~Hz}(25-100 \mathrm{sec}$, Figure 3C), well below the dominant experimental frequency (indicated by the dashed line, Figure 3C; $\sim 0.05 \mathrm{~Hz}, 20.1 \pm 0.3 \mathrm{sec}$, mean interaction length \pm SEM). Critically, this coherence had zero phase-lag, indicating simultaneous in-phase fluctuations across interlocutors, and was significantly stronger within pairs (green line, Figure 3C) than between pairs (black line, Figure 3C). Together, these findings signify a neural coupling that was specific for elements of a communicative pair, driven by temporal synchronization of their BOLD changes, over a time scale spanning several communicative interactions (representative BOLD traces across communicators are shown in Figure 3D). However, differences in coherence are seldom observed without co-occurring changes in spectral power, and therefore might be suspected to be a trivial side-effect. To test this notion we computed the power spectral densities of the signals evoked by the right STG of Communicators and Addressees. As expected, this analysis revealed a predominance of slow fluctuations in this cortical region, but importantly, it also showed that neither the amplitude (Figure $3 \mathrm{~A}$ ) nor the distribution (Figure 3B) of spectral power was affected by within- or across-pair comparisons (as assessed with intersubject correlations of power spectra, Figure 3B), adding emphasis to the validity of the coherence measurements.

Finally, we tested whether the pair-specific temporal synchronization of BOLD changes was driven by simultaneous updating of communicative common ground, specific to epochs involving Novel interactions. This possibility was tested by modeling the STG BOLD signal of each participant separately per interaction epoch (lasting 1 to 3 interactions), thereby obtaining a time series of regressor weights ( 26 for each interaction type), with equal length for each participant, no matter how long their experiment lasted. Single task events (see Figure 1A), and feedback events (separately for positive or negative outcome) were modeled and removed through multiple regression, in order to account for BOLD signal variations not related to the task state within each epoch of Novel interactions. Cross-correlation analyses on time series of task states across pairs revealed an interaction effect of Pair (Within, Between) and Interaction type (Known, Novel), $F(1,1454)=4.6, p=0.032$, see Figure 3E. This interaction was driven by stronger within- than between-pair cross-correlation of the Novel task states, $t(727)=2.0, p=0.044$ (two-sided independent $\mathrm{t}$-test), consistent with stronger within- than between-pair cross-correlation of planning times on Novel interactions (see Supporting Information), and by stronger cross-correlation of the Novel than the Known task states, $t(26)=3.3, p=0.003$ (two-sided paired t-test). There was no statistically significant difference in cross-correlation of between-pair task states (Known vs. Novel). These findings signify that only during the emergence fluctuations of conceptual representations of common ground are coupled across interlocutors (within-pair coherence across Nove task states), but no longer when common ground has already been established and does not need to be adjusted (within-pair coherence across Known task states). 


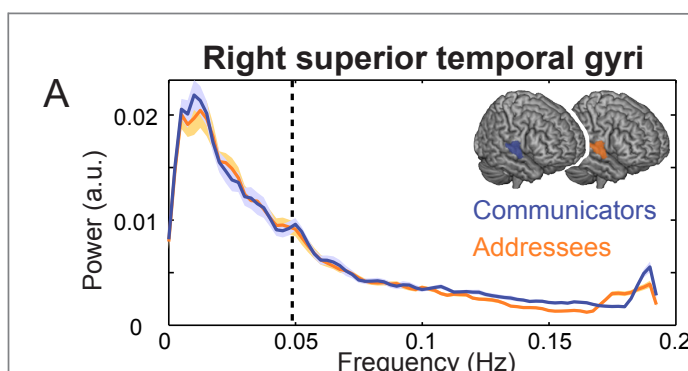

B
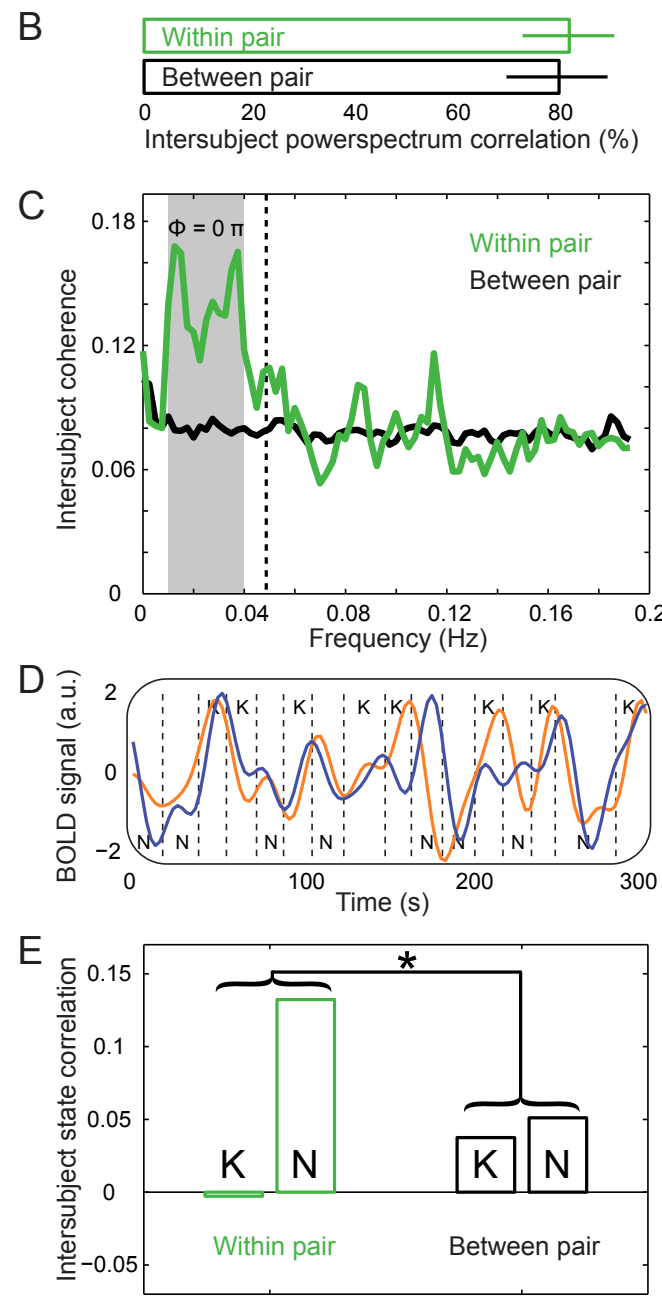

F Left sensorimotor cortices (control)

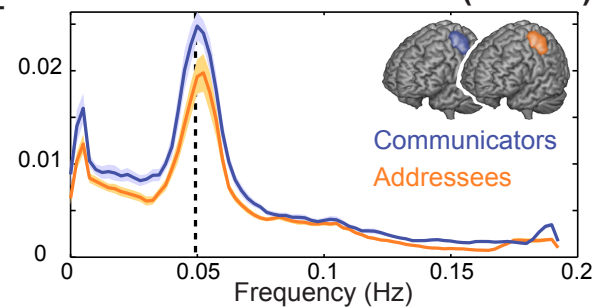

G
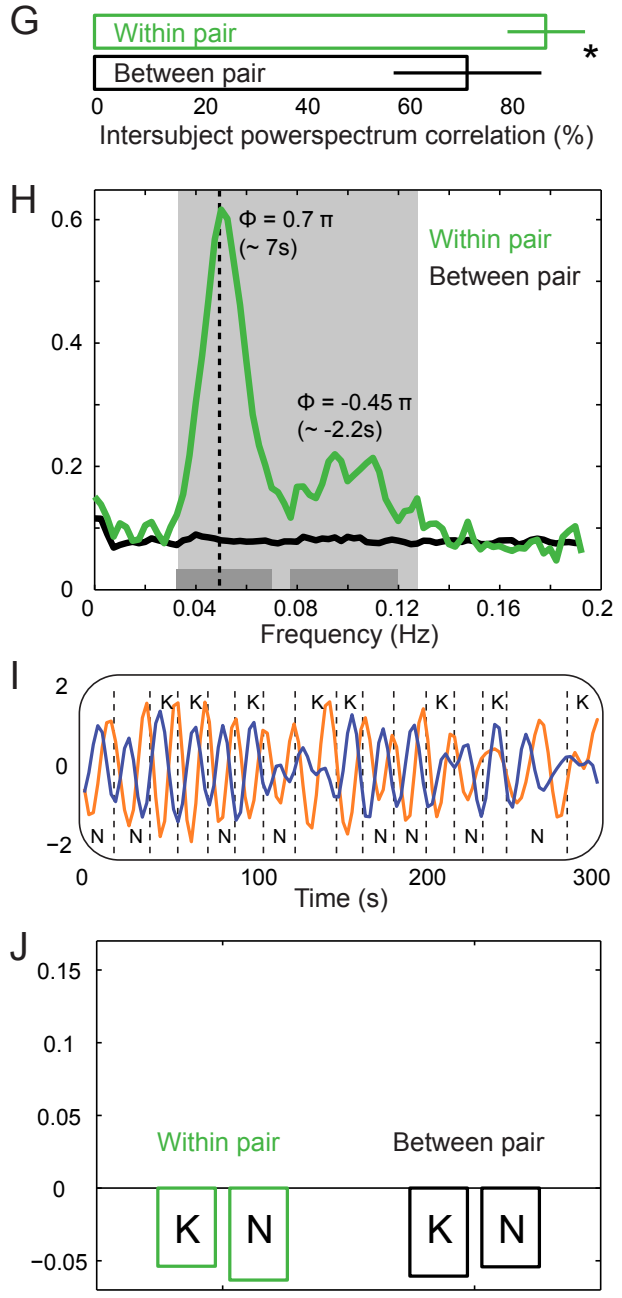

After showing the specificity of these inter-subject coherent neural dynamics for frequency, pairs, and state of common ground, and after testing for potential confounds in spectral amplitude and distribution, we compared the effects observed in right STG to a control region that is a priori expected to show coherency tightly coupled to the experimental structure, the left sensorimotor cortex (MNI [-38, -19, 55]). Indeed, this region showed stronger within- than between-pair coherence, but in contrast to right STG, at $0.05 \mathrm{~Hz}$ with a phase-lag of $0.7 \pi$ ( $7 \mathrm{sec})$. These parameters reflect the dominant experimental frequency and the average temporal gap between = the movements of the participants in a pair (Figure 3F-I). In line with these findings, within-pair coherency of activation in the left sensorimotor cortex was not affected by the cognitive task state of the participants (Known vs. Novel; Figure 3J).

Figure 3. Construction of pair-specific conversational context. (A) Power spectral densities of BOLD signal in right superior temporal gyrus reveal predominantly low frequency content in both Communicators and Addressees. Shades indicate \pm 1 SEM. (B) The frequency content within-pairs was not dissimilar from between-pairs. Error bars indicate $\pm 1 S D$. (C) Coherence spectral densities reveal significantly stronger within- than between-pair temporal synchronization of BOLD changes, with zero phase-lag, and in frequencies lower than the dominant experimental frequency (indicated by dashed line, $\sim 0.05 \mathrm{~Hz}$ ). Grey surface indicates frequencies with a significant difference in coherence $(p<0.05$, corrected for multiple comparisons across frequencies, nonparametric randomization across participant pairs). (D) Example neural traces of a representative pair, band-pass filtered between 0.01 and $0.04 \mathrm{~Hz}$. Dashed lines indicate interaction on- and offsets, where $\mathrm{K}$ and $\mathrm{N}$ denote interaction types, i.e. Known and Novel respectively. (E) Cross-correlations between Communicator and Addressee time series of task states reveal pair-specific conversational context when establishing common ground (Novel interactions). (F) Consistent activation of left sensorimotor cortex at dominant experimental frequency. (G) More similar frequency content within-pairs than between-pairs; asterisk denotes $p<<0.001$. (H) Stronger within- than between-pair coupling at dominant experimental frequency, and higher. Dark grey bars at bottom indicate frequencies with an additional significant difference in phase. (I) Traces of the same pair as in panel D, but band-pass filtered between 0.03 and $0.07 \mathrm{~Hz}$. (J) For sensorimotor cortex, task states are roughly in anti-phase, regardless of presence of common ground and communication. 
Several further control analyses were performed to test alternative interpretations of this finding. First, it could be argued that the intersubject coherence observed in the right superior temporal gyrus is driven by intrinsic ("resting-state") brain activity within the default-mode network (Raichle et al., 2001), rather than by conceptual abstractions related to the communicative interactions. Accordingly, we showed that the effects were robust to removing default-mode resting-state activity, as indexed by timeseries of BOLD signal from the posterior cingulate cortex [(Raichle, et al., 2001), see Figure S3 for voxelwise functional connectivity maps of activity that was regressed out prior to computing coherence]. Second, it could be argued that the intersubject coherence is driven by coherent changes in attention to the auditory stimulation of the MR scanners. Accordingly, we showed that the effects were robust to removing cortical signals related to auditory stimulation, as indexed by timeseries of BOLD signal from the primary auditory cortex [(Bandettini, Jesmanowicz, Van Kylen, Birn, \& Hyde, 1998), see Figure S4 for voxelwise functional connectivity maps]. Third, we tested whether the intersubject coherence spanning several communicative interactions was in fact induced by trial-specific features. Accordingly, we performed the same coherence analysis as above, but this time on a timeseries with trial-specific features regressed out. This was accomplished by constructing an extensive model of task contingencies accounting for BOLD activity driven by each task epoch (see Figure 1A), with feedback events, planning periods of Communicators, and observation periods of Addressees modeled separately for each outcome (Positive, Negative) and interaction type (Known, Novel), leading to a total of nine regressors for each Communicator and Addressee, respectively. The intersubject coherence previously observed between left sensorimotor cortices of Communicator and Addressee was severely affected by this control analysis, resulting in lack of signal power and coherence at the dominant experimental frequency $(\sim 0.05$ $\mathrm{Hz}$, see Figure S5C and D). In contrast, the intersubject coherence observed in the right superior temporal gyrus of Communicator and Addressee remained significant, with stronger within- than between-pair coherences in frequencies lower than the dominant experimental frequency (Figure S5B). This finding emphasizes that intersubject coherence in right STG, unlike left sensorimotor cortex, was driven by abstract features independent from trial-specific elements of the communicative interactions.

\section{Discussion}

This study describes the cortical dynamics supporting the generation of a conceptual space shared across communicators and necessary to understand the symbols used in human referential communication. There are three main findings. First, there was an incremental build-up of common ground within each pair of communicators, as indexed by the gradual increase in joint success when novel communicative problems were tackled over the course of the experiment. This behavioral effect was associated with increasing activation in the right superior temporal gyrus, both in Communicators planning communicative actions and in Addressees observing those actions. Second, the right superior temporal gyrus showed pair-specific temporal synchronization of BOLD changes, at a time scale spanning several communicative exchanges. Third, this pair-specific temporal synchronization was driven by interactions involving novel communicative problems, over and above effects involving the same sensorimotor contingencies, but known communicative problems. Together, these findings suggest that the emergence of meaning, a feature crucial for human communication, relies on conceptual operations shared across interlocutors, contingent on the ongoing interaction, and abstracted away from transient sensorimotor events. This temporal dynamics is not immediately compatible with models of communication that emphasize signal transmission (Hasson, et al., 2012), or predictions driven by individual communicative events (Pickering \& Garrod, 2004, 2013). Rather, the current findings support the notion that conceptual knowledge during a communicative interaction needs to be continuously aligned to the conversational context and to the interlocutor's behavior (Clark, 1996), and that the meaning of a communicative token arises by embedding it in a conceptual space whose activation predates in time the processing of the communicative stimuli themselves (van Berkum, et al., 2008). 


\section{Appendix A}

\section{Supporting Information}

Behavioral data analysis and results

We considered mean planning times (Figure 1B and C), mean movement times, and mean number of moves of Communicator and Addressee within the fMRI session. These dependent variables were calculated for each of the twentyseven pairs of participants and for each of the two problem types (Known, Novel), and compared statistically by means of paired t-tests (two-tailed $\alpha$-level $=0.05$ ). We considered the pairs of participants as the unit of observation for the statistical analysis of task performance (Figure 1D), as the communicative task performance is dependent of both elements of a pair. Participants jointly solved the interactions well above chance level $(71 \pm 2 \%$ correct, mean \pm SEM; conservative estimate of chance level: $1 / 8$ th, 8 potential target locations, neglecting the potential orientations). They solved more Known (94 $\pm 1 \%$, mean \pm SEM) than Novel interactions (47 $\pm 4 \%) ; t(26)=13.6, p<0.001$ Figure 1D. In a similar vein, the participants planned longer (Communicators: $t(26)=5.9, p<<0.001$; Addressees: $t(26)=8.5, p<<0.001$, Figure $1 \mathrm{~B}$ and C), moved longer (Communicators: $t(26)=8.1, p<<0.001$; Addressees: $t(26)$

$=13.2, p<<0.001$ ), and made more moves (Communicators: $t(26)=3.1, p$ $<.005$; Addressees: $t(26)=17.7, p<0.001)$ within the Novel interactions than within the Known interactions. These findings indicate that the Known interactions were easier than the Novel interactions; most likely because of the different types of problems faced by the participant pairs within these interaction types, and because these interactions were completed before during the training session outside the scanners (see Experimental procedures in the Materials and Methods)

Given our prediction that neural activity changes with establishing common ground within a pair, we investigated the change in joint task performance on the Novel interactions over the course of the experiment. Both a linear and a logarithmic function were considered to describe this change. The linear function turned out to be good fit, $F(1,40)=35.2, p<<0.001, \mathrm{R}^{2}{ }_{\text {adj }}=.46$, but the change was best approximated by a logarithmic function, $F(1,40)=51.6, p$ $<<0.001, \mathrm{R}^{2}{ }_{\text {adj }}=.55$ (see the green curve in Figure 1D). We chose to use this logarithmic function as a parametric time modulation of specific task regressors in the first level fMRI analysis (see fMRI image acquisition and analysis in Materials and Methods). Neither function significantly approximated the changes in joint performance on the Known interactions (both $F s<1.8$ ).

Previous work showed stronger within-interaction coupling between Communicator and Addressee planning times than between players of a 
non-communicative control interaction (Stolk, Verhagen, et al., 2013). This observation suggests that the novel communicative problems encountered by the participants evoked stronger mutual adjustments between pairs than the non-communicative problems. Here, we performed a similar cross-correlation analysis, separately for planning times of Communicators and Addressees evoked during Known and Novel interactions. In the Novel interactions, the within-interaction coupling between Communicator and Addressee planning times was stronger within pairs $(r=0.15 \pm 0.18$, mean \pm SD) than between pairs $(r=0.06 \pm 0.16 ; t(727)=2.8 ; p=0.006$, two-sided independent $t$-test $)$. There was no such significant difference on the Known interactions (withinpairs: $r=0.09 \pm 0.17$; between-pairs: $r=0.06 \pm 0.17$ ). This observation suggests that a Novel communicative problem was concomitantly more difficult for both Communicators and Addressees of the same pair.

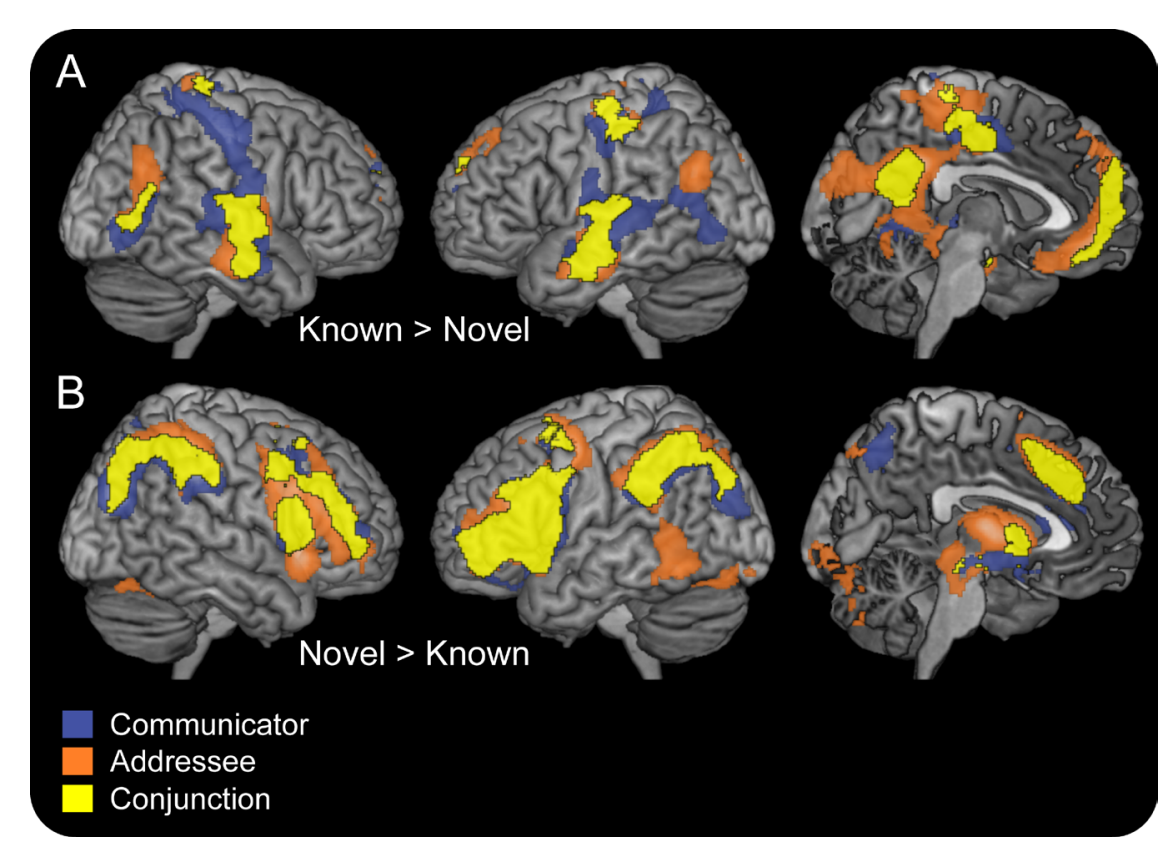

Supplemental Figure 1. Imaging results for the main effect of familiarity (panel A, Known > Novel), and novelty (panel B, Novel > Known) on neural activity evoked during planning (Communicators, epoch 2 in Figure 1A) and observing communicative actions (Addressees, epoch 3). The conjunction shows the commonalities in neural activities between the two roles. The statistical maps are thresholded at $p<0.05$, whole-brain FWE-corrected, see Table S1 for details.

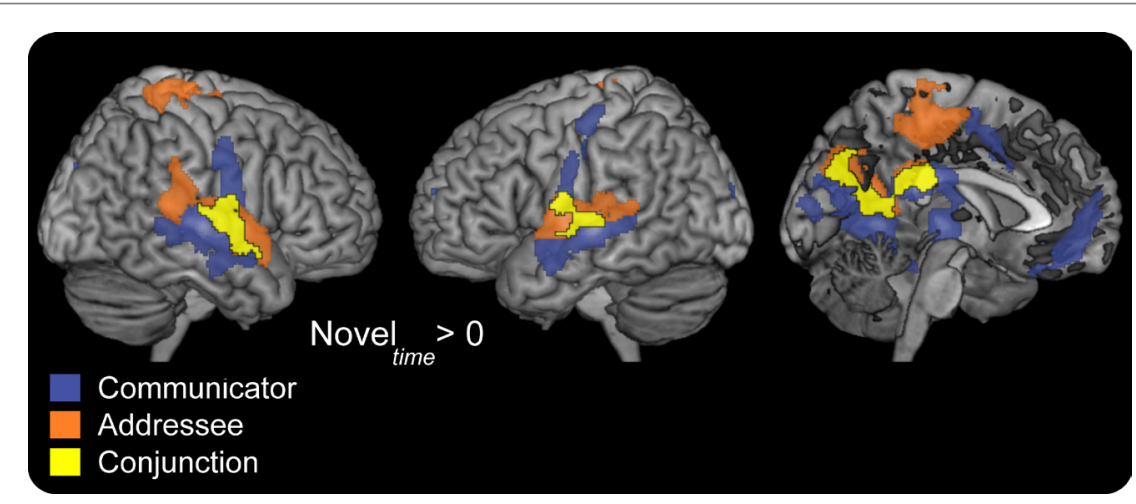

Supplemental Figure 2. Brain regions showing increasing activity with increasing joint performance on the Novel interactions over the course of the experiment (see green curve in Figure 1D), evoked during the planning (Communicators, epoch 2 in Figure 1A) and observation of communicative actions (Addressees, epoch 3). The statistical maps are thresholded at $p<0.05$, whole-brain FWE-corrected, see Table S1 for details. As expected on basis of the behavioral data, the time modulations of activity evoked during the epochs of the Known interactions did not yield any overlapping activity patterns between the two roles (not shown). 


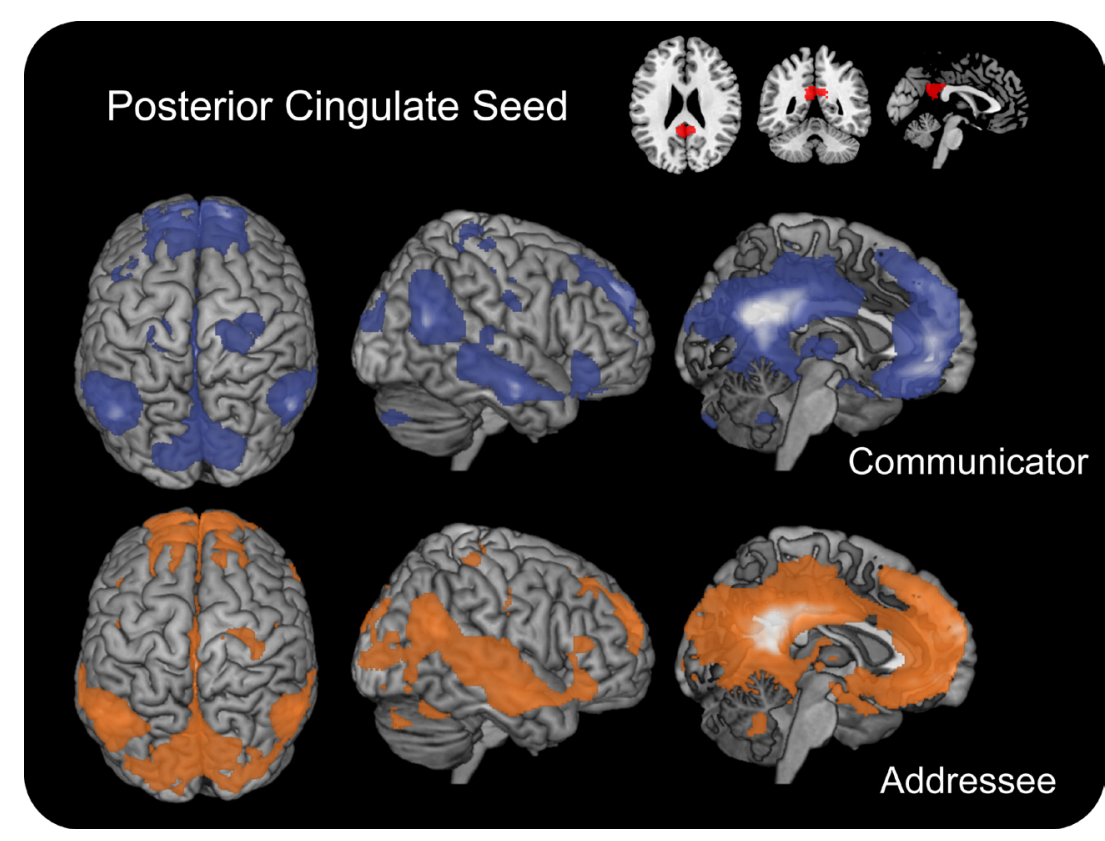

Supplemental Figure 3. Voxelwise functional connectivity maps of posterior cingulate cortex (PCC), separately for Communicators and Addressees. The statistical maps are thresholded at $p<0.05$, whole-brain FWE-corrected. The posterior cingulate was individually defined for each participant on the basis of the spatial overlap between the PCC AAL-template ((Tzourio-Mazoyer et al., 2002), indicated in red in top right) and the participant's segmented grey matter, to ensure minimal spatial overlap between the template and CSF or white matter. The PCC time course was calculated by averaging across all voxels within the seed region.

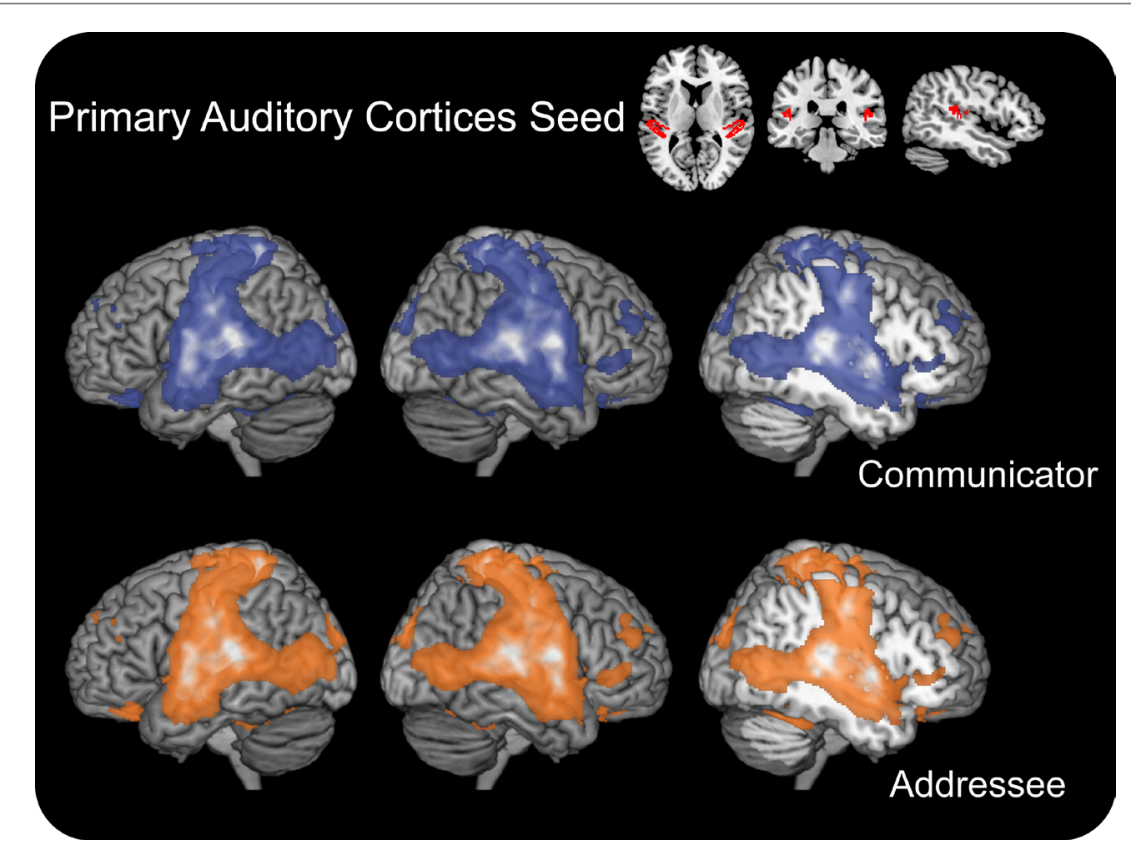

Supplemental Figure 4. Voxelwise functional connectivity maps of primary auditory cortices (Brodmann Area 41), separately for Communicators and Addressees. The statistical maps are thresholded at $p<0.05$, whole-brain FWE-corrected. The primary auditory cortices were individually defined for each participant on the basis of the spatial overlap between the Brodmann Area 41 AFNI-template ((Morosan et al., 2001), indicated in red in top right) and the participant's segmented grey matter, to ensure minimal spatial overlap between the template and CSF or white matter. The primary auditory cortices time course was calculated by averaging across all voxels within the seed region. 

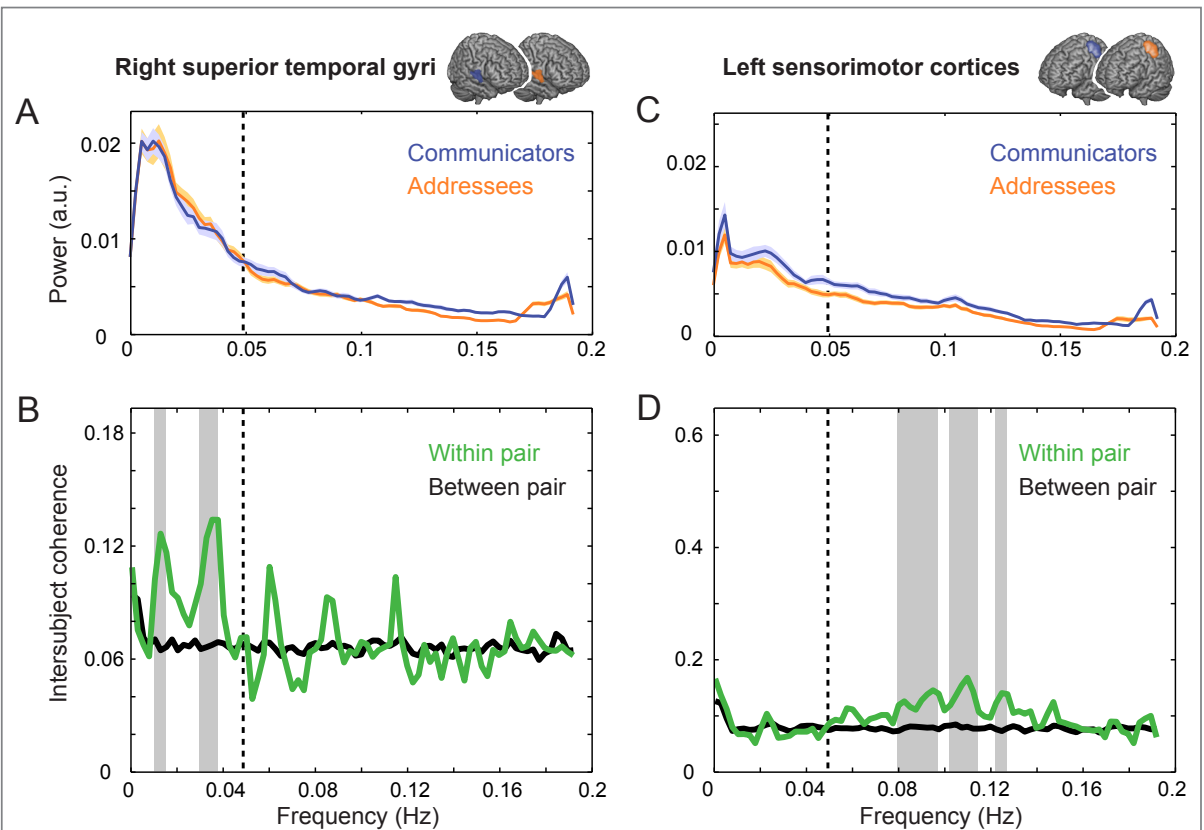

Supplemental Figure 5. Power spectral density and coherence spectra of whole-experiment BOLD signals as in Figure 3, but with additional modeling and regressing out of activity attributed to the separate task epochs (Figure 1A). As compared to Figure 3 , left sensorimotor cortices now lack signal power and coherence at the dominant experimental frequency $(\sim 0.05 \mathrm{~Hz}$, panels $\mathrm{C}$ and $\mathrm{D})$. In contrast, the right superior temporal gyri remained showing significantly stronger within- than between-pair coherences in frequencies lower than the dominant experimental frequency (panel B). Grey surfaces indicate frequencies with a significant difference in coherence $(p<0.05$, corrected for multiple comparisons across frequencies, nonparametric randomization across participant pairs).

Supplemental Table 1. Results of the random effects analysis related to planning (Communicators) and observing (Addressees) Known and Novel communicative actions.

\begin{tabular}{|c|c|c|c|c|c|c|}
\hline \multirow[t]{2}{*}{ contrast } & \multirow[t]{2}{*}{ Region } & \multirow[t]{2}{*}{ Cluster size } & \multicolumn{3}{|c|}{ MNI coordinates } & \multirow[t]{2}{*}{ t.value } \\
\hline & & & $x$ & $\mathrm{x}$ & $z$ & \\
\hline \multirow[t]{5}{*}{ Communicator: Known > Novel } & Right precentral gyrus/ Insula lobe & 33957 & 4 & -20 & 23 & 6.7 \\
\hline & Posterior cingulate cortex & ${ }^{3134}$ & -3 & .53 & 19 & 5.5 \\
\hline & Mid orbital gyrus & 2466 & -1 & 55 & 13 & 4.6 \\
\hline & Leff middle temporal gyrus & 1697 & -48 & -65 & 9 & 4.5 \\
\hline & Right middle temporal gyus & 1612 & 48 & -65 & ${ }^{3}$ & 5.0 \\
\hline \multirow[t]{5}{*}{ Communicator: Novel > Known } & Leff anterior cingulate cortex Infereriof fronat byus & 23315 & -24 & 18 & 17 & 9.1 \\
\hline & Left supramargrinal gyus & 17703 & 4 & -57 & 39 & 8.2 \\
\hline & Right middle frontal gyrus & 4794 & 35 & 21 & 34 & 7.0 \\
\hline & Right inferior fronatal gyrus & 1977 & 51 & 15 & 10 & 6.2 \\
\hline & Right cerbellum & ${ }^{924}$ & 21 & -75 & -30 & 4.9 \\
\hline \multirow[t]{3}{*}{ Communicator: Novel...., > $>0 "$} & Leff middele temporal gyrus & ${ }^{5003}$ & .50 & .20 & ${ }_{12}$ & 7.6 \\
\hline & Right superior temporal gyrus & 4395 & 53 & -14 & 12 & 7.1 \\
\hline & Mid orbital gyrus & 693 & -4 & 52 & -1 & 5.0 \\
\hline \multirow[t]{5}{*}{ Addressee: Known $>$ Novel } & Posterior cingulate cortex & 27949 & -15 & -34 & 25 & 9.0 \\
\hline & Mid orbital gyrus & 8073 & -3 & 46 & 6 & 9.0 \\
\hline & Right superior temporal gyrus/Insula lobe & 7496 & 43 & -12 & ${ }^{2}$ & 6.2 \\
\hline & Right middle temporal gyrus & ${ }^{2263}$ & 46 & -59 & 17 & 5.9 \\
\hline & Leff middle emporal gyrus & 1583 & -45 & -62 & 26 & 7.4 \\
\hline \multirow[t]{8}{*}{ Addressee: Novel > Known } & Left pallidum/Inferior frontal gyrus & 28974 & -22 & 16 & 17 & 10.3 \\
\hline & Right inferior fronatal gyrus & 12747 & 34 & ${ }^{21}$ & -5 & 7.9 \\
\hline & Left supramarginal gyus & 6920 & -40 & .54 & 39 & 7.7 \\
\hline & Right supramarg inal gyus & 5906 & 40 & -53 & 44 & 6.1 \\
\hline & Left cerebellum & 3529 & -20 & -77 & -21 & 5.6 \\
\hline & Right cerebellum & 2311 & 21 & .72 & -27 & 7.8 \\
\hline & Left middle emporal gyrus & 1513 & -56 & -51 & -3 & 5.6 \\
\hline & Leff precuneus & ${ }^{617}$ & -7 & -68 & 47 & 4.9 \\
\hline \multirow[t]{3}{*}{ Addressee: Novel $\operatorname{lime}_{\text {mex }}>0^{*}$} & Right superior temporal gyus Insula lobe & 1560 & 50 & -13 & ${ }^{11}$ & 5.6 \\
\hline & Right precuneus & 970 & ${ }^{11}$ & -57 & 18 & 6.3 \\
\hline & Leff sperior remporal gyus & 504 & -56 & -10 & ${ }^{5}$ & 6.2 \\
\hline \multirow[t]{7}{*}{ Conjunction: Known $>$ Novel } & Right superior temporal gyrus/ Insula lobe & 5159 & 44 & -12 & 3 & 5.7 \\
\hline & Left superior remporal gyus & 4987 & -44 & -18 & 1 & 5.2 \\
\hline & Posterior cingulate cortex & 2461 & 0 & -53 & 26 & 5.5 \\
\hline & Middle cingulate cortex & 2327 & 7 & -21 & 54 & 4.9 \\
\hline & Left postcentral gyrus & 2205 & -34 & -24 & 51 & 4.2 \\
\hline & Mid orbital gyrus & 2064 & -1 & 55 & 12 & 4.4 \\
\hline & Right middle emporal gyus & 566 & 47 & -64 & 7 & 3.7 \\
\hline \multirow[t]{8}{*}{ Conjunction: Novel> > Known } & Leff inferior frontal gyrus & 14297 & -33 & 22 & 22 & 8.4 \\
\hline & Leff supramargrinal gyus & 4507 & -42 & .54 & 39 & 7.8 \\
\hline & Right supramargrginal gyrus & 4235 & 41 & .55 & 42 & 5.8 \\
\hline & Right middle frontal gyrus & 3215 & 37 & ${ }^{21}$ & 33 & 5.8 \\
\hline & Right inferior frontal gyrus & 1782 & 52 & 16 & ${ }^{11}$ & 5.8 \\
\hline & Leff putamen & 1395 & -11 & 3 & -1 & 7.1 \\
\hline & Right caudate nucleus & 1236 & ${ }^{11}$ & 4 & ${ }^{2}$ & 5.8 \\
\hline & Right cerbellum & 704 & 20 & .74 & .30 & 4.9 \\
\hline 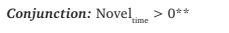 & Right superior temporal gyrus & 829 & 58 & -8 & 5 & 4.4 \\
\hline
\end{tabular}



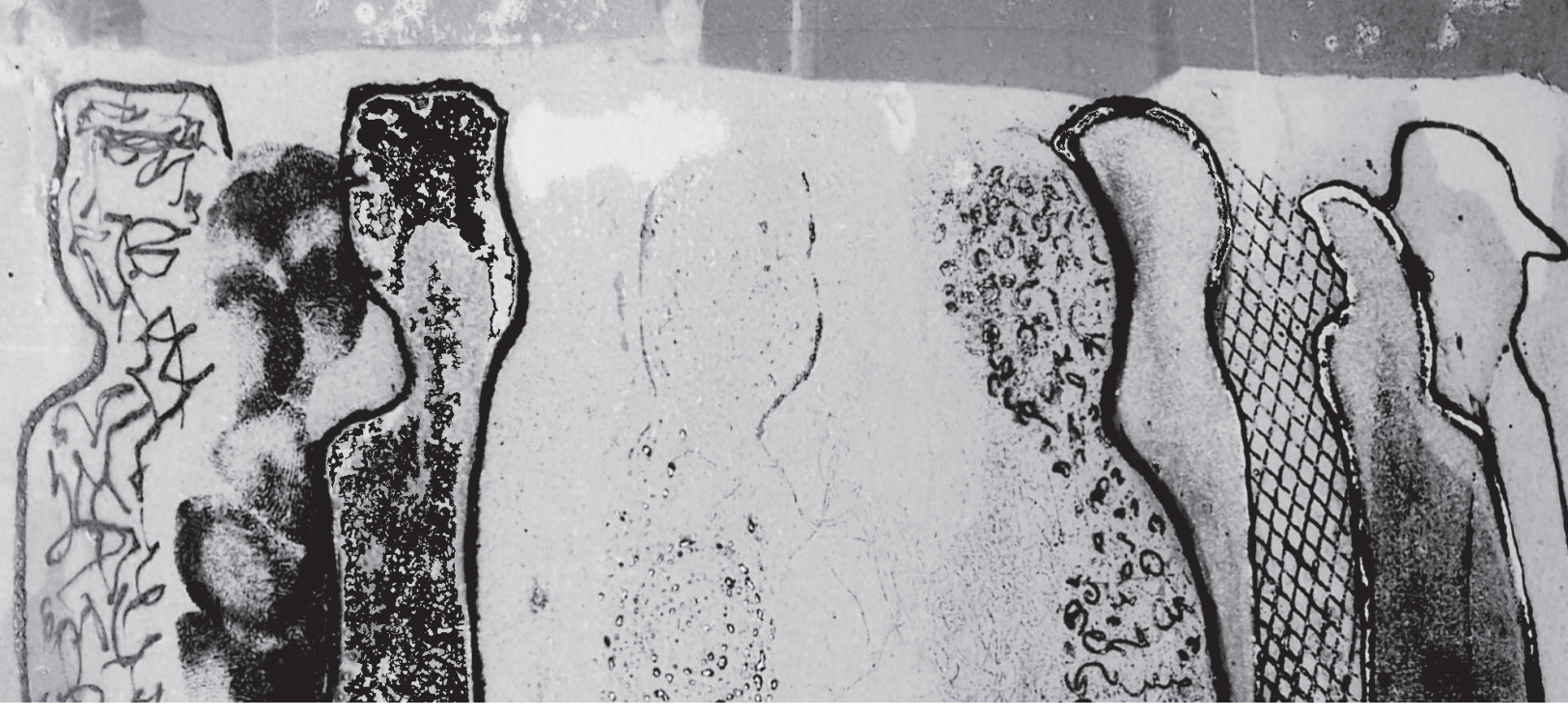

\author{
Interfering with human \\ communication
}




\section{Understanding communicative actions: a repetitive TMS study}

Stolk A, Noordzij ML, Volman I, Verhagen L, Overeem

S, van Elswijk G, Bloem B, Hagoort P, Toni I 


\section{Introduction}

Human referential communication involves selecting behaviors that allow an addressee to recognize the communicative intentions of those behaviors (Levinson, 2006). In everyday communication, we largely exploit pre-established shared symbols built in a common language to make those intentions accessible to our interlocutors. Yet, even within such a conventional symbol system, those intentions still need to be inferred from multiple semantic ambiguities present in every utterance (Levinson, 2006). This study aims at characterizing a neural mechanism that supports the inferential processes required for human referential communication (Cutica, Bucciarelli, \& Bara, 2006; Noordzij, et al., 2009; Sabbagh, 1999).

Given that human referential communication rides on a large background of pragmatic inferences, several authors have started to study these inferential abilities under situations in which shared symbols are not available and their occurrence can be experimentally controlled (de Ruiter, et al., 2010; Galantucc \& Garrod, 2011). This work has shown that the right posterior superior temporal sulcus (pSTS) is an important element of the cerebral system supporting human referential communication, both for communicators generating novel signals as well as for addressees trying to decode those signals (de Langavant et al., 2011; Gao, Scholl, \& McCarthy, 2012; Mashal, Faust, Hendler, \& Jung-Beeman, 2007; Noordzij, et al., 2009, 2010). However, it remains to be seen whether unperturbed functioning of pSTS is necessary for inferring the intentions of communicative behaviors, in particular when those intentions cannot be retrieved on the basis of conventional symbols. Here, we address this issue by temporarily disrupting neural function in the right pSTS by using low-frequency repetitive transcranial magnetic stimulation (rTMS), in the context of a task requiring participants to infer the meaning of novel referential communicative behaviors. We aim at qualifying the nature of the right pSTS contributions to communicative inferences by analyzing the alterations caused by a transient interference with neural activity in this region.

The involvement of the right pSTS in establishing a novel referential communicative system is one among several contributions associated with this region, including the perception of biological motion and goal directed actions, moral judgments, and mental state attribution (Arfeller et al., 2013, Bahnemann, Dziobek, Prehn, Wolf, \& Heekeren, 2010; Grossman, Battelli, \& Pascual-Leone, 2005; Shultz, Lee, Pelphrey, \& McCarthy, 2011). This heterogeneity might reflect superficial differences of an underlying unitary function. The right pSTS might generate predictions based on the integration 
of current sensory stimuli with domain-independent priors (Jakobs et al., 2012; Schultz, Friston, O'Doherty, Wolpert, \& Frith, 2005), as suggested by its involvement with predictions pertaining to different domains [e.g. body schema (Blanke et al., 2005; Grossman, et al., 2005), gravity (Bosco, Carrozzo, \& Lacquaniti, 2008), and beliefs (Young, Camprodon, Hauser, Pascual-Leone, \& Saxe, 2010)]. Although this predictive function could be an instance of a general Bayesian inference mechanism (Friston, 2010; Friston, Kilner, \& Harrison, 2006), the pSTS appears to be distinctively able to construct predictions based on information from several different categories. This property appears particularly well suited for handling the domain-independent abductions that are required when producing and interpreting novel symbols (Fodor, 2000; Quine, 1960). We reasoned that those predictions could capture 1) sensory predictions based on statistical regularities of the sensory stimuli experienced by the participants, as implied in some accounts of human communication (Iacoboni, 2005; Schippers, et al., 2010; Tognoli, Lagarde, DeGuzman, \& Kelso, 2007; Turesson \& Ghazanfar, 2011); 2) conceptual predictions based on semantic conventions, as established by the participants during the experiment (Schultz, et al., 2005; Wyk, Hudac, Carter, Sobel, \& Pelphrey, 2009; Young, et al., 2010); or 3) conceptual predictions based on a dynamic context shared among communicators, as determined by the trial-by-trial adjustments of the participants to the intended meaning of the stimuli (Menenti, et al., 2012). The first possibility would suggest that rTMS over the right pSTS alters performance across different tasks that use the same timeseries of sensory stimuli. The second possibility would suggest rTMS-related alterations that are a function of the overall level of proficiency in a communication task. We indexed proficiency as the number of correct responses per unit time (Efficiency, (Machizawa \& Driver, 2011; Nixon, Lawton-Craddock, Tivis, \& Ceballos, 2007; Townsend \& Ashby, 1983; Woltz \& Was, 2006)). The third possibility would suggest rTMS-related alterations that depend on the recent history of communicative interactions of the participants. We indexed these dynamic adjustments in communicative proficiency as the rate of change in Efficiency over trials (Efficiency Rate).

In this study, participants' abilities to comprehend novel communicative actions were quantified in a controlled and validated experimental setting; the Tacit Communication Game (TCG; Figure 1A) (Blokpoel, et al., 2012; de Ruiter, et al., 2010; Newman-Norlund, et al., 2009). In this interactive task, two players are asked to recreate a spatial configuration of two simple geometrical shapes (one for the Communicator and one for the Addressee) on a digital game board. Crucially, this spatial configuration is shown to the Communicator only (trial epoch 1 in Figure 1A). This requires the Communicator to convey, and the Addressee to comprehend, the position and orientation of the Addressee's shape.
The only means the players had to communicate is with an unconventional tool, namely by moving their simple geometrical shape. Different pairs of participants solve these novel communicative problems in different ways (Blokpoel, et al., 2012; de Ruiter, et al.,2010), an indication that pairs mutually converge on a common solution from a potentially infinite set of possible arbitrary solutions (van Rooij, et al., 2011). This feature of the task makes it possible to isolate adjustments in communicative performance driven by the recent history of interactions between participants from overall variations in communicative performance, and their interaction with stable cognitive traits. Those traits are an important source of inter-subject variance in communicative abilities. For instance, Addressee's performance on the Raven's progressive matrices test (Raven, 1989, 2000) predicts how quickly a communicative pair establishes novel shared symbols (Volman, et al., 2012). Accordingly, we used this psychometric index to characterize the inferential processes supported by the right pSTS during referential communication. Namely, Addressees with high Raven's scores might more readily use abstract relations to quickly generate novel analogical mappings between observed actions and their communicative intentions (Blokpoel, et al., 2012; Carpenter, Just, \& Shell, 1990; Volman, et al., 2012). We reasoned that if rTMS over right pSTS influences Addressees' ability to quickly grasp novel communicative meanings according to recent communicative interactions (see Hypothesis \#3 above), then Addressees with high Raven's scores might be particularly impaired by rTMS-induced cerebral alterations.

Specificity of the rTMS intervention was ensured by controlling for the communicative relevance of the stimuli and for the cerebral location of the intervention, across four different experimental sessions. Functionally, we assessed the effects of rTMS during a visual tracking task that used exactly the same timeseries of stimuli shown during the communication task, but with no communicative requirements. Anatomically, we contrasted the effects of stimulating the right pSTS with those evoked by stimulating a contiguous homotopic region involved in integrating position information when viewing moving objects [left MT+, (Bosco, et al., 2008; Maus, Ward, Nijhawan, \& Whitney, 2012)], a function required for processing the stimuli used during the communication and the visual tracking tasks. 


\section{Materials and methods}

\section{Participants}

Thirteen right-handed healthy adults (18-28 years, mean $=22$; seven women) with normal or corrected-to-normal vision participated in this study. Participants were screened for contra-indications of TMS and gave written informed consent according to the institutional guidelines of the local ethics committee (Committee on Research Involving Human Subjects, Region Arnhem-Nijmegen, The Netherlands). They received written instructions for each experimental task and were either offered a financial payment or given credits towards completing a course requirement as a compensation for their participation.

\section{Experimental design}

Participants performed two tasks: a communication task and a visual tracking task. The task that the participants were currently performing was indicated throughout the relevant trials on the participants' screen. Each task involved two players, i.e. a participant and a co-player, two geometrical shapes ("tokens"), and two hand-held game controllers. In both tasks the participants observed the movements of the co-player's token, namely horizontal translations, vertical translations or 90 degrees clockwise rotations on a visually presented $3 \times 3$ digital grid (the "game board"). The co-player was facing another 19 inch monitor in the same sound-proof experimental room. The participants and the co-player were not otherwise allowed to interact with each other.

During the communication trials both players had to jointly reproduce a spatial configuration of two tokens presented to the co-player only (trial epoch 1 in Figure 1A). The participant observed the communicative actions of the co-player, the Communicator, to infer the target position and orientation of his token (epoch 2). A yellow bar signaled the end of the Communicator's movements and indicated that the participant's turn had started. The participant (the Addressee) had unlimited time to plan his actions (but was instructed to plan as fast as possible, epoch 3). After pressing the start button, the participant had five seconds to move his token from the center grid position towards the inferred target position and orientation (epoch 4). When the participant pressed the start button again (and within a maximum movement time of five seconds), feedback was presented to both players to indicate whether they had jointly reproduced the target configuration (epoch 5).

During the visual tracking trials, the participant observed the token's movements on the grid, as during the communication trials. However, the participant was told that in these tracking trials the co-player was instructed to move across predetermined grid locations. The task of the participant was to move his token to the grid location last visited twice by the co-player before she completed her movements. Grid locations where the co-player rotated her token twice were also considered as "visited twice". After moving to the grid location last visited twice, the participants were asked to rotate their token twice on that location. If the co-player had not visited or rotated at any location twice during her movements, the participant had to visit the center grid location and rotate twice. After five seconds or when he pressed the start button, feedback was presented to indicate whether the participant had successfully completed the trial according to the task instructions.

Unbeknownst to the participants, the co-player was a confederate that was only pretending to control her token with the game controller. In fact, during both tasks the co-player actions were identical, namely pre-recorded token movements reproducing frequently used strategies identified in previous studies involving this task setting (Blokpoel, et al., 2012; de Ruiter, et al., 2010; Noordzij, et al., 2009). For instance, in case the Communicator's token was orientation specific (i.e. a rectangle or triangle, but not a circle), the Communicator went to the Addressee's target position, rotated her token to indicate the target orientation of the Addressee's token, and then moved to her own target position completing her part of the target configuration. In case her token was a circle, the Communicator first went to the Addressee's target position and briefly waited. Then she wiggled a couple of times (e.g. repeating the number 2 action in trial epoch 2 - Figure 1A) to indicate the target orientation of the Addressee's token, and then moved to her own target position. Occasionally, she did not wiggle when such an action was redundant given a situation in which the target orientation matched that of the begin orientation of the receiver token.

An experiment consisted of 4 sessions (Figure 1B) spread over 2 separate days ( $\sim 2$ weeks separation) and lasted about 5 hours in total. An initial familiarization block preceded the two sessions on the first day ( $\sim 35$ minutes) and a re-familiarization block those on the second day ( $\sim 5$ minutes). Each session encompassed 80 trials ( $\sim 15$ minutes) organized by type (Communication, Visual tracking) into 8 blocks of 10 trials (Figure 1B). The participants alternated between the two types on a block-by-block basis with the order counterbalanced across participants. Two sessions with no prior stimulation were used to determine pre-stimulation and post-stimulation baselines. These sessions took place $\sim 1$ hr before session 2 and $\sim 1$ hr after session 3 respectively. The participants received low-frequency rTMS over right pSTS or left MT+ $(\sim 20$ minutes, Figure 1C) just prior to task performance in sessions 2 and 3 which were recorded on separate days. For each participant the moment of the two 
TMS interventions was matched for the time of the day ( $\pm 1.5 \mathrm{hr}$ ). The order of stimulation site was counterbalanced across participants.

\section{Transcranial magnetic stimulation protocol}

The pulses were administered offline, i.e. prior to task performance, to induce a temporally stable and task-independent modulation of local cortical processing efficiency. The stimulation sites in the right pSTS and left MT+ were selected on the basis of the peak voxel (group contrast, Communicative $>$ NonCommunicative) reported in a previous fMRI study with the communication task (right pSTS, MNI coordinates: [50, -42, 14], (Noordzij, et al., 2009)), and on the basis of the mean spatial location reported in a cytoarchitectonic analysis of the human extrastriate cortex (left MT+, [-43, -70, 10], (Malikovic et al., 2007)). Restricting the stimulation of this control area to the opposite hemisphere as our experimental site of interest minimizes the possibility of cortical spread across stimulation sites as a result of the repetitive stimulation (Paus et al., 1997)

High-resolution anatomical images were acquired using a MP-RAGE sequence on a separate day (176 slices, TE/TR $=3.68 / 2.25 \mathrm{~s}$, voxel size $1 \times 1 \times 1 \mathrm{~mm}$ ). These images were spatially normalized to standard MNI space using SPM2 (Wellcome Department of Imaging Neuroscience, London, UK) and used to calibrate a frameless stereotactic system (BrainSight, Rogue Research Inc.) linking each participant's structural scan and stimulation sites. By means of neuronavigation the TMS coil was positioned over the relevant brain location (either right pSTS or left MT+). TMS was then delivered using a biphasic Magstim Super Rapid Stimulator (Magstim Company Ltd, Whitland, UK), using a figure-of-eight coil with a diameter of $70 \mathrm{~mm}$. We applied a 20 minute train of repetitive low-frequency $(1 \mathrm{~Hz})$ stimulation at $60 \%$ maximum stimulator output (Grossman, et al., 2005). Previous studies have shown that $1 \mathrm{~Hz}$ stimulation temporarily reduces metabolic activity by $5-30 \%$ (Mottaghy et al., 2002; Valero-Cabre, Payne, \& Pascual-Leone, 2007) and excitability of the cortex within the stimulated area (Boroojerdi, Prager, Muellbacher, \& Cohen, 2000).
A

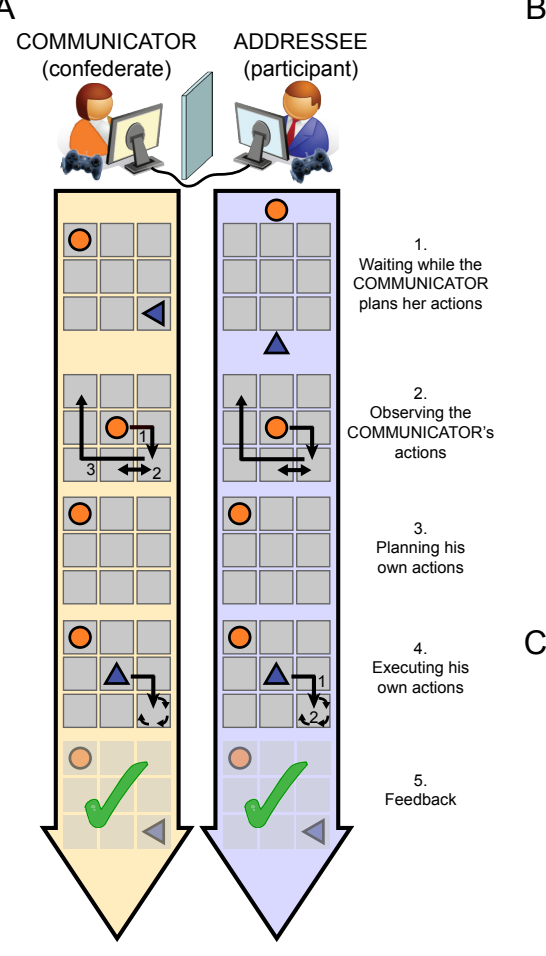

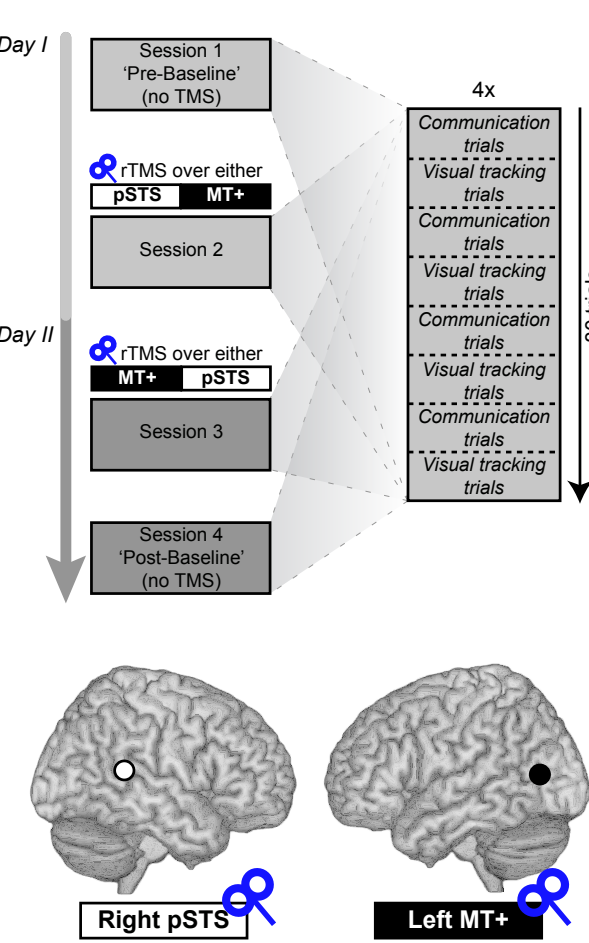

Figure 1. Task setup. (A) Example communication trial in which both players had to jointly reproduce a spatial configuration of two tokens presented to the first player in turn only, i.e. the Communicator (epoch 1). A participant, the Addressee, had to infer from the Communicator's actions (epoch 2, orange token, starting at the center) where and how to position his token (blue). During a visual tracking trial involving the same sequence of events (not shown), the participant viewed identical actions but with the instruction to determine the grid location last visited twice or rotated at by his co-player. (B) The experiment consisted of 4 sessions spread over 2 separate days. Participants received TMS at $1 \mathrm{~Hz}$ for 20 minutes just prior to task performance in sessions 2 and 3 . The order of stimulation sites was counterbalanced across participants. Each session encompassed 80 trials organized by type (Communication, Visual tracking) into 8 blocks of 10 trials and with the order counterbalanced across participants. (C) Whole-brain visualization of stimulation sites; right pSTS (white dot, MNI coordinates: [50, -42, 14]) and left MT+ (black dot, [-43, -70, 10]). 


\section{Cognitive traits}

A recent study (Volman, et al., 2012) shows that the ability of a pair of communicators to establish novel shared symbols is predicted by the Communicator's score on the Need for Cognition test (NCS, (Cacioppo, Petty, \& Kao, 1984)) and by the Addressee's score on the Raven's progressive matrices test (Raven, 1989, 2000). The score on the Raven test is a non-verbal measure of fluid reasoning, in which participants solve up to 36 problem items with increasing difficulty in 20 minutes. Each item of this test consists of three series of three images. The last of the three series lacks the third image and has to be filled out by the participant choosing from one of eight options. Solving problems in the Raven test is thought to capture cognitive processes related to encoding and inferring regularities in the test items. This test is thought to distinguish individuals on their ability to induce abstract relations and to handle a large set of problem-solving goals in working memory (Carpenter, et al., 1990). Accordingly, we reasoned that if rTMS over right pSTS influences Addressees' ability to quickly grasp novel communicative meanings according to recent communicative interactions (see Hypothesis \#3 in the Introduction), then Addressees with high Raven's scores might be particularly impaired by rTMS-induced cerebral alterations. Namely, Addressees with high Raven's scores might more readily use abstract relations to generate novel mappings between observed actions and their communicative intentions (Blokpoel, et al., 2012; Carpenter, et al., 1990; Volman, et al., 2012). Participants' Raven's scores were therefore expected to account for a significant portion of inter-individual variability in task performance during the communication trials (Volman, et al., 2012), both before and after rTMS. To assess the specificity of the cognitive traits captured by the Raven, we also asked the Addressees tested in this experiment to complete the Need for Cognition questionnaire (NCS, (Cacioppo, et al., 1984), translated in Dutch), a personality questionnaire consisting of 18 statements targeting participants' intrinsic motivation to solve cognitive challenges. The psychometric assessments were completed by the participants on the second day, between the third and fourth session.

\section{Data analysis}

Planning times and accuracies on both tasks were recorded by Presentation software (Neurobehavioral Systems, Albany, CA, USA) and analyzed offline using custom MATLAB code (MathWorks, Natick, MA, USA). Participants' planning time was defined as the interval in seconds starting after the Communicator's movement interval and ending when the participant pressed the start button (epoch 3 in Figure 1A). Accuracy refers to the percentage of successfully accomplished trials. In order to consider the combined effects of both measures of task performance, we used Efficiency (i.e. Accuracy/Planning time; see (Machizawa \& Driver, 2011; Nixon, et al., 2007; Townsend \& Ashby, 1983; Woltz \& Was, 2006) and Efficiency Rate (d(Efficiency)/d(trials)). Efficiency increases with greater accuracies and smaller planning times and it indicates the number of correct responses per unit time. Efficiency Rate indicates the rate of change in Efficiency over trials, i.e. the benefits from task experience gained during preceding trials in a session, calculated as the beta value of a linear regression of Efficiency across the 40 task-specific trials of each experimental session. To ensure that the data adheres to the assumption of normality, we filtered the planning times and accuracies (which is a binomial measure) with a moving average of 7 trials prior to estimating the regression. This approach allows a robust estimate of the overall (linear) trend across an experimental session that is sensitive to sustained changes in performance rather than singletrial errors. A positive rate indicates an improvement in task performance over trials.

We analyzed the effect of experimental manipulations on Efficiency and Efficiency Rate in two steps. First, we tested whether participants improved their performance in either task over the course of the experiment. Showing changes in performance between the pre- and post-stimulation baseline sessions excludes that floor or ceiling effects prevent the detection of rTMS-related effects in task performance in the intervening stimulation sessions (Figure 1B). Accordingly, the effects of Task (Communication, Visual tracking) and Time (Pre-, Post-stimulation baseline) on Efficiency were estimated using a $2 \times 2$ repeated-measures analysis of variance (ANOVA). Second, we tested whether rTMS over the right pSTS influenced overall task performance (Efficiency) and within-session changes in task performance (Efficiency Rate). In order to avoid spurious differences between sessions with and without rTMS intervention, we focused this analysis on sessions 2 and 3, i.e. sessions with prior rTMS intervention. Accordingly, the effects of Task (Communication, Visual tracking) and TMS site (pSTS, MT+) on Efficiency and Efficiency Rate were estimated using a $2 \times 2$ repeated-measures analysis of covariance (ANCOVA). The covariates in this analysis considered the inter-subject variance accounted for by the order of stimulation (e.g. session 2: pSTS; session 3: MT+; or viceversa) and cognitive traits (i.e. mean-centered psychometric scores on Raven and NCS, see the Cognitive traits section). By adding the interaction terms between the covariates and the within-subject variables to the model we test whether the hypothesized within-subject interaction between Task and TMS site is affected by each subject covariates scores (Anstey et al., 2007; Delaney \& Maxwell, 1981). The significant results $(p<.05)$ are reported. 


\section{Results}

Prior to any stimulation participants were able to successfully accomplish each task well above chance level (conservative estimate of chance level over 9 game board locations: 11\%). The percentage of correct responses at pre-stimulation baseline was $85 \pm 3 \%$ and $86 \pm 3 \%$ (mean \pm standard error of the mean) for the communication task and the visual tracking task respectively.

We compared task performance before and after the rTMS intervention (prebaseline and post-baseline sessions, see Figure 1) to assess the presence of overall learning effects. A repeated-measures analysis of variance on Efficiency revealed main effects of Task (Communication, Visual tracking; $F(1,12)=5.2, p$ $=.042$, effect size partial $\eta^{2}=.30$.) and Time (Pre-, Post-stimulation baseline; $F(1,12)=25.1, p<0.001$, partial $\eta^{2}=.68$.). These results indicate that the visual tracking task (mean Efficiency over both baseline sessions: 0.85), was more difficult than the communication task (mean Efficiency: 0.96), and that the participants became more proficient over the course of the experiment (see Figure 2). Crucially, there was no interaction effect between Task and Time factors on the Efficiency index. This finding suggests that the overall learning rates from initial baseline (session 1) to final learned performance (session 4) of the two tasks are well comparable. This result allows for an unbiased assessment of whether Efficiency and Efficiency Rate during task learning (sessions 2 and 3) are affected by TMS perturbation.

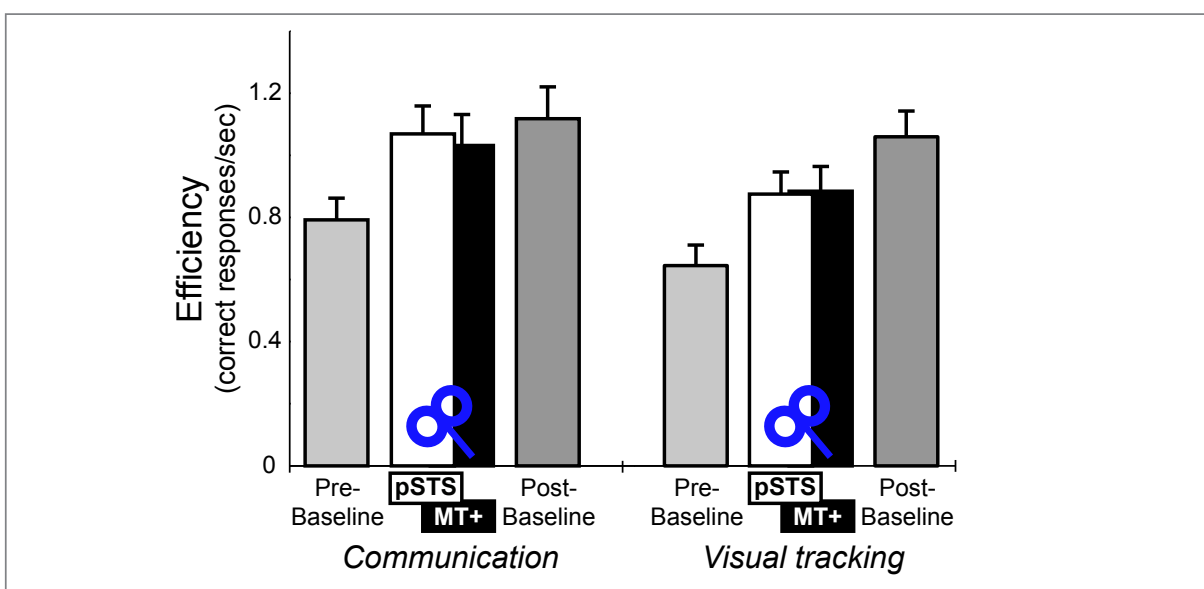

Figure 2. Group results for Efficiency on the communication and visual tracking task. Participants became more proficient at each task over the course of the experiment. There was no interaction of Task and TMS site (right pSTS, left MT+). Error bars indicate 1 standard error of the mean.
We then assessed the influence of rTMS-induced cerebral alterations on the level of task performance during sessions 2 and 3, as captured by the measure of Efficiency, and on the ability to benefit from task experience gained during preceding trials in a same session, as captured by the Efficiency Rate. A repeatedmeasures analysis of covariance on Efficiency (covariates: order of stimulation site, Raven and NCS scores) revealed a main effect of Task (Communication, Visual tracking), $F(1,9)=11.8, p=.008$, partial $\eta^{2}=.57$, and an interaction effect of TMS site (pSTS, MT+) and stimulation order, $F(1,9)=8.0, p=.020$ partial $\eta^{2}=.47$. These results indicate that the two tasks differed in complexity also in the rTMS sessions, and that the order of rTMS intervention (session 2 : pSTS; session 3: MT+; or viceversa) had a strong impact on mean performance across both tasks.

Importantly, following TMS to the pSTS, there was less improvement over trials (smaller Efficiency Rate) in the communicative setting than following TMS to MT+ or over visual tracking trials, $F(1,9)=6.4, p=.032$, partial $\eta^{2}=.42$. Thus, the interaction between Task (Communication, Visual tracking) and TMS site (pSTS, MT+) did not have an effect on Efficiency (Figure 2), but it did have an effect on the Efficiency Rate (see Figure 3A). Furthermore, this interaction effect was affected by a participant's score on the Raven test, as indicated by an interaction of Task, TMS site, and Raven, $F(1,9)=5.6, p=.042$, partial $\eta^{2}$ $=.39$. There were no other statistically significant main or interaction effects, all $p>.12$.

In a post-hoc analysis of the simple effects constituting this interaction we observed a strong negative association between the Raven test score and the Efficiency Rate at the communication task, $\mathrm{r}_{\mathrm{s}}=-.695, p=.008$, but not at the visual tracking task following TMS over pSTS, $r_{s}=.169, p=.6$ (see Figure 3B), as indicated by Spearman's rank-order correlation. Further post-hoc exploration of the relation between Raven's scores and performance in both tasks indicated that there was a statistically significant positive correlation between the Raven test score and the Efficiency Rate at the communication task during the pre-stimulation baseline, $r_{s}=.657, p=.015$. This result indicates that individuals that quickly grasped novel communicative meanings according to recent communicative interactions before receiving rTMS, were also most affected by rTMS over pSTS during the communication task. There were no other significant correlations between the Raven and the Efficiency Rate, or the Efficiency, across any of the other sessions. 


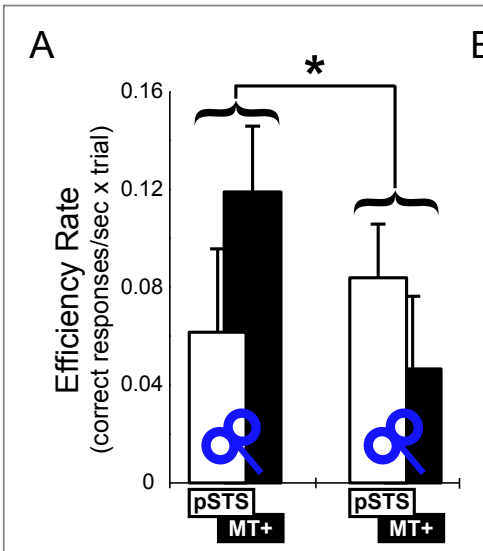

B

Communication Visual tracking

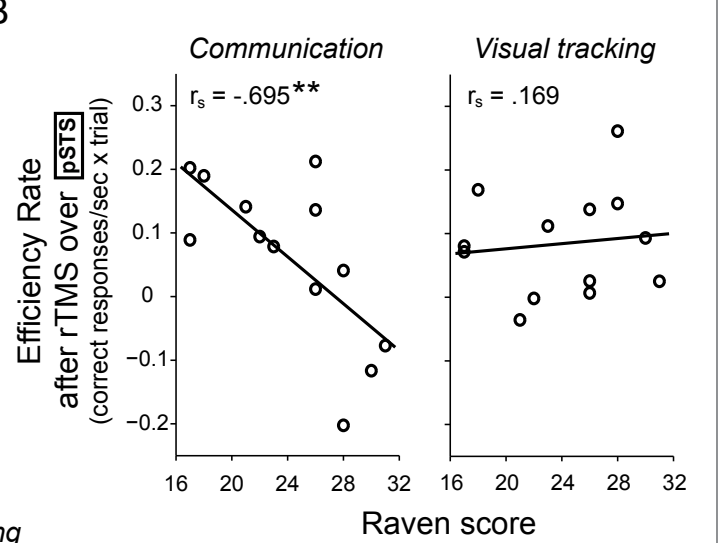

Figure 3. Group results for Efficiency Rate on the communication and visual tracking task. (A) Efficiency Rate on the communication and visual tracking task with prior rTMS. A positive rate indicates an improvement in task performance over trials. Asterisk (*) indicates a significant interaction between Task (Communication, Visual tracking) and TMS site (right pSTS, left MT+) on the Efficiency Rate ( $p<$ $.05)$. Error bars indicate 1 standard error of the mean. (B) Scatter plots of individuals Raven's score against Efficiency Rate during performance of the communication and the visual tracking task, following rTMS over right pSTS. Black line: least-square regression line; rs: Spearman rank correlation coefficient; ** $p<.01$.

\section{Discussion}

This study was designed to investigate whether a functionally intact right pSTS is necessary for understanding the intention of novel communicative actions. We used an experimental setting where an Addressee needs to disambiguate communicative and instrumental components of the movements of a communicative partner, and find a relation between the communicator's movements and their meaning. Mimicking the movements of the communicative partner (Hasson, et al., 2012; Tognoli, et al., 2007) or following low-level statistical regularities in the stimulus material (Pickering \& Garrod, 2004) are not viable options for solving this task. Rather, the absence of pre-existing conventions encourages the Addressee to use higher-order conceptual structures when inferring meaning from the observed movements (van Rooij, et al., 2011). We targeted the right pSTS given that previous studies indicated increased metabolic activity in this region when subjects made those communicative inferences (Noordzij, et al., 2009). There are two main results. First, rTMS over the right pSTS reduced participants' ability to improve their task efficiency during the course of the communicative interactions. This impairment of Efficiency Rate was functionally and anatomically specific. Functionally, the impairment occurred in relation to corresponding effects in a visual tracking task that used exactly the same sequences of stimuli. Anatomically, the impairment occurred in relation to rTMS effects on a contiguous homotopic temporal region (left MT+). Second, the magnitude of the rTMS effect over right pSTS was particularly strong in those Addressees with high Raven's scores. The analogical movement-meaning mappings established by those participants might be less sensitive to the continuously changing surface structure of the communicative problems (Volman, et al., 2012). This observation suggests that alterations of the right pSTS are particularly disruptive for those participants that under normal circumstances process the movements of their communicative partner according to abstract relations. Taken together, these observations indicate that the contribution of the right pSTS to communicative inferences is dynamic and conceptual in nature. This finding qualifies the suggestion that the right pSTS integrates current sensory stimuli with internalized contextual priors (Jakobs, et al., 2012; Schultz, et al., 2005), by showing that this region is involved in updating higher-order predictions on sensory material according to the recent history of communicative interactions. 


\section{Interpretational issues}

It could be argued that the significant within-session effect on communicative performance (as indexed by the Efficiency Rate) should have given rise to a detectable between-sessions effect on Efficiency, i.e. reduced Efficiency between pre- and post-stimulation baseline sessions. However, this subtle effect is likely lost in the large between-sessions variance (partly owing to the order of stimulation site) and unspecific improvements in efficiency across sessions. Accordingly, we cannot completely exclude that the pSTS plays a role in specifying predictions based on invariant meanings of the communicative stimuli (see Hypothesis \#2 in the Introduction).

The analyses focused on those sessions preceded by rTMS interventions and with comparable tasks experience. The differential effects observed between pSTS and MT+ stimulation on performance of the communication and visual tracking task raise the question of how the stimulation effects compare to a sham or null baseline session involving the same experience with the tasks. The existing baseline sessions (sessions 1 and 4) cannot be used for this comparison, given that these were not matched for experience with the tasks. An additional (fifth) session without rTMS might have been included in the experimental design, but we refrained from doing so given that it is debatable whether a null or a sham session can provide an interpretable control for TMS studies (Drager, Breitenstein, Helmke, Kamping, \& Knecht, 2004). For instance, incidental effects of TMS (stimulation noise, somatosensation on the head) have been found to influence task performance in multiple ways (Duecker \& Sack, 2013). Future experiments using neurostimulation techniques with more selective sham controls (e.g. transcranial direct current stimulation, (O'Shea et al., 2013)) might be able to better address this issue.

It could be argued that the strong improvements in performance of the communication task during the first experimental session (pre-stimulation baseline) caused participants with relatively high Raven's score to reach a performance ceiling. Accordingly, the relative alteration of their communicative behavior in the subsequent session would be a consequence of that performance ceiling, rather than of the rTMS intervention. Two observations argue against this possibility. First, task performance continued to improve in the post-stimulation baseline (session 4, see Figure 2). Second, if participants with high Raven scores were at ceiling level after the first session, while participants with relatively ow Raven scores still had room for improvement on the communication task, then there should have been a negative relation between the Raven scores and Efficiency Rate when MT+ was stimulated. In fact, this relation was absent, and there were clear improvements in communicative performance across the group when MT+ was stimulated. Accordingly, the disruption in communicative performance observed in participants with high Raven's scores is more consistent with the notion that alterations of the right PSTS are particularly disruptive for those participants that under normal circumstances process the movements of their communicative partner according to abstract relations.

It could be argued that the relative decline in Efficiency Rate of the communication task after rTMS over pSTS is un-specific, since a similar decline was observed in the visual tracking task after rTMS over MT+. In fact, this finding reinforces the notion that pSTS effects are specifically tuned to sensory stimuli processed in a communicative framework, since the two tasks used exactly the same stimuli. By the same token, this finding emphasizes that MT+ is also involved in predicting stimulus characteristics (and changes thereof) relevant for the visual tracking task (Beintema \& Lappe, 2002; Sterzer, Haynes, \& Rees, 2006), for instance movement patterns that can be used to predict which grid location is visited twice (see Figure 1)

\section{Relevance for human communication}

Although the current findings were obtained under experimental conditions that purposely limited the availability of pre-existing shared symbols among communicators, we believe that this work is relevant for understanding the contribution of the right pSTS to a fundamental property of human communication, namely how humans rapidly create those shared symbols from scratch (Evans \& Levinson, 2009; Tomasello, 2008). The ability to quickly build new semiotic conventions and re-configure existing ones emerges at different levels of human communication, from infants learning a language without access to the local communicative conventions, to adults disambiguating semantic relations according to pragmatic cues (Egidi \& Caramazza, 2013; van Berkum, et al., 2008). Even during a simple conversation, we continuously update and sharpen conceptual predictions on sensory material according to the recent history of the communicative interaction (Menenti, et al., 2012). The present findings increase our understanding of the neural mechanisms of human communication by showing that the right pSTS is necessary for continuously adjusting those priors according to the recent history of interactions of the communicators, over and above the statistical regularities of the sensory stimuli experienced by the participants. This suggests that human communicative abilities operate on conceptual inferences, rather than sensorimotor brain-tobrain couplings (Hasson, et al., 2012), and that those conceptual inferences are continuously updated. This suggestion fits well with the temporal dynamics of neural activity observed in this region, namely context-dependent neuronal upregulation emerging already before the occurrence of communicative 
stimuli, and further transient responses to incoming visual information (Stolk, Verhagen, et al., 2013). It remains to be seen whether the right pSTS supports the dynamic updating of communicative inferences also when communication relies on linguistic material with strongly established semantic conventions (Mitchell, Ames, Jenkins, \& Banaji, 2009; van Ackeren, Casasanto, Bekkering, Hagoort, \& Rueschemeyer, 2012; Willems et al., 2010).

\section{Conclusion}

This study uses repetitive transcranial magnetic stimulation to investigate the necessity and nature of the contributions of the right posterior superior temporal sulcus to human communication. After interference with neural activity in this region, the communicative abilities of addressees were reduced. This impairment was confined to their ability to benefit from recent communicative experiences. These findings qualify the contributions of the right pSTS to human communicative abilities, showing that this region might be necessary for incorporating previous knowledge, accumulated during interactions with a communicative partner, to constrain the inferential process that leads to action understanding. 


\section{Altered communicative adjustments following ventromedial prefrontal lesions}




\section{Introduction}

It has been repeatedly shown that our communicative actions are selected according to what we believe addressees of a communicative interaction know and believe [audience design, (Clark, 1996; Holler \& Stevens, 2007; Jacobs \& Garnham, 2007; O'Neill, Bard, Linnell, \& Fluck, 2005)]. For instance, adults tend to modify their speech, gestures, and accompanying body motions when addressing a child (Brand, Baldwin, \& Ashburn, 2002; Brodsky, Waterfall, \& Edelman, 2007; Grieser \& Kuhl, 1988; O’Neill, et al., 2005; Warrenleubecker \& Bohannon, 1984). Even the mere belief of interacting with a child leads us to produce more emphatic communicative actions (Newman-Norlund, et al., 2009; Stolk, Hunnius, Bekkering, \& Toni, 2013). Yet, little is known about the cerebral mechanisms supporting this cognitive skill. We reasoned that if audience design is an instance of our ability to adjust actions to the presumed knowledge and beliefs of others, then the ventromedial prefrontal cortex (vmPFC) should be involved in adjusting referential communicative behaviors to a mental model of an addressee (Amodio \& Frith, 2006; Behrens, Hunt, Woolrich, \& Rushworth, 2008; Mitchell, Macrae, \& Banaji, 2006; Stolk, Verhagen, et al., 2013).

The ventromedial prefrontal cortex (vmPFC) has been consistently implicated in supporting behaviors guided by a mental model of the social world. Yet, patients with vmPFC lesions appear to consider the presumed knowledge of their interlocutor during verbal interactions. It remains unclear whether that performance reflects linguistic phenomena, or genuinely preserved communicative abilities. Here we quantify non-verbal referential interactions in 8 patients with vmPFC damage, 8 patients with brain damage outside vmPFC, and 15 healthy controls. Participants were asked to communicate non-verbally with two different addressees, a child or an adult, in an interactive online setting. In reality, a confederate acted as both child and adult addressees, with matched performance and response times, such that the two addressees differed only in terms of the communicator's beliefs. VmPFC patients communicated as effectively as lesion- and healthy-controls, but failed to adjust their communicative behavior to the addressee's performance and presumed abilities. These findings indicate that following a lesion to the ventromedial prefrontal cortex, humans are still motivated and able to select and alter effective communicative actions, but those actions are not guided by a mental model of their current communicative partner. This observation confirms that the ventromedial prefrontal cortex is involved in selecting behaviors guided by a mental model of the social world, and it suggests a cerebral dissociation between selecting an effective communicative action and adjusting the manner of that action to the presumed characteristics of the addressee.
Patients with vmPFC lesions [often involving the neighboring orbitofrontal cortex as well (Zald \& Andreotti, 2010)) are more strongly guided by the immediate outcome of an event than by mental models of reality, e.g. by future consequences of their behavior, beliefs, social norms and stereotypes (Bechara, Damasio, Damasio, \& Anderson, 1994; Beer, John, Scabini, \& Knight, 2006; Ciaramelli, Braghittoni, \& di Pellegrino, 2012; Ciaramelli, Muccioli, Ladavas, \& di Pellegrino, 2007; Ciaramelli, Sperotto, Mattioli, \& di Pellegrino, 2013, Kaczmarek, 1984; Milne \& Grafman, 2001; Shamay-Tsoory, Tomer, Berger, Goldsher, \& Aharon-Peretz, 2005; Stone, Baron-Cohen, \& Knight, 1998). For instance, moral judgements of vmPFC patients are more strongly influenced by the outcome of an harmful action than by its underlying intention, i.e. whether the harm was attempted or accidental (Ciaramelli, et al., 2012). Here we test whether the inability of vmPFC patients to use a mental model of the social world impairs their non-verbal communicative abilities.

We focus on non-verbal communication since it is known that damage to the vmPFC does not modify patients' use of shorter utterances and definite references following repeated verbal interactions, a linguistic behavior assumed to rely on the ability to understand another's knowledge (Gupta, Tranel, \& Duff, 2012). However, communicative interactions based on verbal materia might by-pass mental models of an interlocutor and approximate normal behavior through increased accessibility of syntactic and semantic structures 
recently used in a communicative interaction (Sass, Krach, Sachs, \& Kircher 2009; Segaert, Menenti, Weber, Petersson, \& Hagoort, 2012). Accordingly, here we test whether the inability of vmPFC patients to use a mental model of an interlocutor becomes evident once communicative interactions are not confounded with linguistic phenomena.

Audience design effects were quantified in a controlled experimental setting involving the production of referential non-verbal behaviors with a communicative goal (de Ruiter, et al., 2010), exploiting a previously validated protocol (Newman-Norlund, et al., 2009; Stolk, Hunnius, et al., 2013). Given the novel communicative situation experience by the participants, the behaviors evoked in this two-player game could not be directly based on previous concrete experiences or on pre-established conventions (e.g. a common language). The task features allowed us to directly tap into patients' ability to spontaneously generate communicative adjustments to their mental model of an addressee. Participants were told they were playing an online interactive game with an adult or a child, in alternation. In fact, a confederate performed the role of both addressees, while remaining blind to which one the two roles she was performing in any given trial. Accordingly, both performance and response time of the two presumed addressees were matched.

Communicative behaviors of vmPFC patients were compared to the behaviors of a brain-lesion control group without vmPFC alterations (LC, matched on lesion extent to the vmPFC group), and of a healthy control group (HC, matched on gender, age, and education to the other groups). We also used 'voxel-based lesion-symptom mapping' (VLSM) to obtain a data-driven index of the anatomical relationship between lesion location and communicative adjustments (Bates et al., 2003; Rorden \& Karnath, 2004), independently from pre-established patients' classifications.

\section{Materials and Methods}

\section{Participants}

Participants $(\mathrm{N}=32)$ included 17 patients with brain damage, and 15 healthy controls (HCs), recruited at the Centre for Studies and Research in Cognitive Neuroscience, Cesena, Italy. The patients were selected on the basis of the location of their lesion as assessed on MRI or CT scans. One patient was excluded from data analysis due to poor task performance, as indicated by disproportionately slow movements (with mean total movement time per trial of $48 \mathrm{~s}$, compared to a $5 \mathrm{~s}$ sample population mean). Eight patients had lesions centered on the ventromedial prefrontal cortex (vmPFC, see Figure 1), consequences of the rupture of an aneurysm of the anterior communicating artery. Eight lesion controls (LC) were selected on the basis of having a cerebral vascular damage that did not involve the ventromedial prefrontal cortex. Six of these patients had a left unilateral lesion (covering parts of occipital, temporal, parietal, or subcortical areas), one patient had a right unilateral lesion (temporaloccipital area), and one patient had a lesion that extended bilaterally (occipital area). All patients were screened for hemiparesis, neglect, and for unobstructed performance and perception of the task (in case of hemianopia, lesion control group). The healthy control group (HC) comprised 15 individuals matched to patients with vmPFC lesions on gender, age, and education (see Table 1). Al participants gave informed consent, according to the Declaration of Helsinki and the Ethical Committee of the Department of Psychology, University of Bologna.

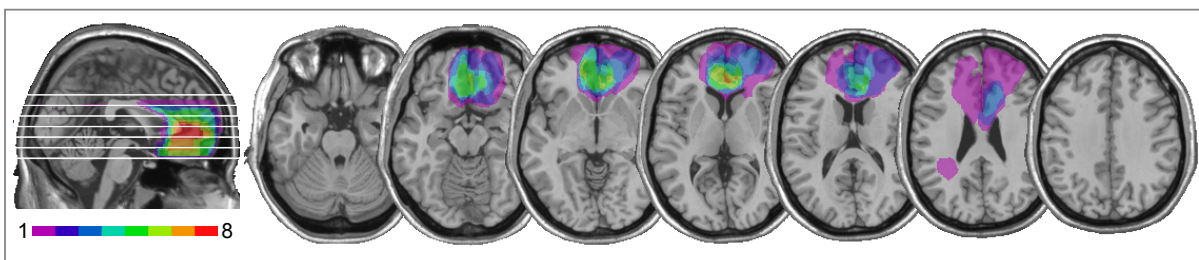

Figure 1. Location and degree of overlap of brain lesions in eight patients with ventromedial prefrontal cortex (vmPFC) damage. The color bar indicates the number of overlapping lesions. Maximal overlap occurs in Brodmann areas 10, 11, and 32. 
Table 1. Participant groups' demographic and clinical data.

$\begin{array}{lllll}\text { Group } & \text { Sex (M/F) } & \text { Age (years) } & \text { Education (years) } & \text { Lesion volume (cc.) } \\ \begin{array}{l}\text { vmPFC } \\ (\mathrm{N}=8)\end{array} & 8 / 0 & 55(9) & 11(4) & 43(18) \\ \mathrm{LC} & 6 / 2 & 63(8) & 12(7) & 59(22) \\ (\mathrm{N}=8) & p=.13 & p=.07 & p=.70 & p=.16 \\ \mathrm{HC} & 13 / 2 & 58(12) & 11(5) & -(-) \\ (\mathrm{N}=15) & p=.28 & p=.42 & p=.80 & \\ \text { LC = lesion controls; HC = healthy controls; } \mathrm{F}=\text { female; } \mathrm{M}=\text { male; the values in parentheses are standard deviations; }\end{array}$

$\mathrm{LC}=$ lesion controls; $\mathrm{HC}=$ healthy controls; $\mathrm{F}=$ female; $\mathrm{M}=$ male; the values in $\mathrm{pa}$
statistical inferences are based on independent samples t-test (chi-squared test for gender)

\section{Lesion analysis}

For each patient, lesion extent and location was documented by using the most recent CT or MRI scans. Patients' lesions were reconstructed onto templates from the Montreal Neurological Institute with MRIcro software (Rorden \& Brett, 2000). Superimposing each patient's lesion onto the template brain allowed us to estimate the total brain lesion volume. The location of lesions was identified by overlaying the lesion area onto the Automated Anatomical Labeling template provided with MRIcron. Figure 1 shows vmPFC patients' location and degree of overlap of brain lesions.

\section{Communication task}

We used the same communication task as employed and described in previous experiments (Newman-Norlund, et al., 2009; Stolk, Hunnius, et al., 2013). The game involves a Communicator (the participant, displayed as a bird on the game board) and an Addressee (the confederate, displayed as a squirrel) interacting on a digital game board with a $3 \times 3$ grid layout. On each trial, their joint goal was for the Addressee to collect an acorn from the game board, located on any of the fifteen white circles (see Figure 2A). Given that knowledge of the acorn's location in the game board was available to the Communicator only (on a printed copy of the game board, visible throughout the trial), a successful trial of this game required the Communicator to inform the Addressee where the acorn was located. Given the experimental setup, the Communicator could inform the Addressee only by moving the bird across the game board (Figure 2A). The Addressee could then move the squirrel to the acorn's location only by interpreting the meaning of the Communicator's movements on the game board (Figure 2A).
By touching a square on the screen with his/her finger, the Communicator could move the bird token to that square, and this movement was also visible to the Addressee. The bird could only move to the center of each of the nine grid squares, and only through vertical or horizontal displacements. This feature of the task was introduced to create a spatial disparity between the movements of the bird and the potential locations of the target object (any of the fifteen

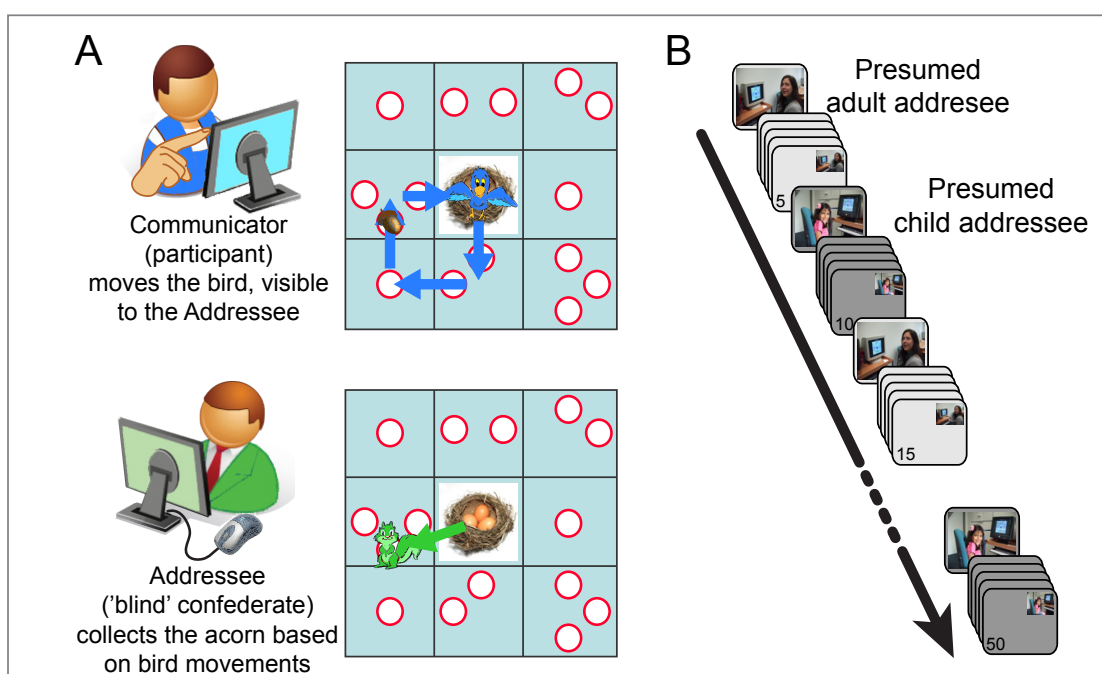

Figure 2. (A) The joint goal of the Communicator and Addressee is for the Addressee to collect the acorn from the digital game board. Given that knowledge of the acorn's location in the game board is available to the Communicator only (on a printed copy of the game board), a successful trial requires the Communicator to inform the Addressee, by virtue of movements of a bird on the game board, where the acorn is located. By touching a square on the screen with his/her finger, the Communicator can move the bird to that square, and this movement was also visible to the Addressee. However, the bird can only move to the center of each of the nine grid squares, and only through vertical or horizontal displacements. This feature of the task makes it difficult for the Communicator and the Addressee to discriminate the location of multiple potential targets within a square (the white circles) on the basis of the location of the bird alone. The Addressee has no spatial restrictions on the movements of the squirrel on the game board. (B) Participants were informed that they would be playing the game, as Communicators, with another adult and a 5-year-old child, in alternation. A digital photograph of the current presumed Addressee was presented to the communicator in full screen before the onset of each block of 5 trials, and in the top right corner during each block. In fact, a confederate performed the role of both Addressees, while remaining blind to which one of the two roles he was performing in any given trial. 
white circles). The Communicator had no restrictions on planning time or on movement time. The end of the movement epoch was marked by the return of the bird on the central square of the game board (nest). At this point, the token of the Addressee (the squirrel) appeared in the center of the digital game board, visible to both players. The Addressee moved the squirrel to the location deemed appropriate given the movements of the Communicator. The Addressee had no temporal or spatial restrictions on the movements of the squirrel on the game board. Successful trials, in which the Addressee had moved to the location of the target, resulted in the presentation of a large acorn on the screen. A red "no" icon was presented over a small acorn for unsuccessful trials.

Task difficulty was manipulated by introducing a disparity between the movements allowed to the bird token (i.e. translations to the center of each square) and the potential location of target object (i.e. the white circles). Namely, the bird could not be overlaid on the precise location of the acorn when a square contained more than one white circle. Importantly, even in trials where the acorn was located in a square with one white circle, the participant needed to find a way to make clear to the Addressee which square contained the acorn, disambiguating it from other squares on the game board, in particular from those squares visited by the bird while moving across the game board. Trials where the acorn was located in a square with one, two, or three white circles were pseudo-randomly intermixed such that there was an overall increase in difficulty during the course of the experiment. The rationale for manipulating task difficulty was to challenge the participants toward the creation of new communicative behaviors.

\section{Experimental procedure}

During the experiment, the participant sat in an experimental room, facing a monitor displaying the digital game board. An experimenter sat next to the participant, providing the task instructions and the trial-specific location of the target object (i.e. an acorn), but playing no part in the communicative game. A confederate sat in another room facing another monitor, showing the same game board seen by the participant. First, each participant was familiarized with the experimental setup ( 5 trials, $\sim 5 \mathrm{~min}$ ). During these familiarization trials the participant was encouraged to freely move the bird around the game board, experiencing the constraints on the bird's movements as described in the task section. The participant was also instructed to return the bird character to the nest (center game board location) at the end of his movements. This task requirement was emphasized by a continuous sound (a bird squeak) that started when the participant moved away from the nest and stopped when he returned the bird to the nest.
Second, each participant was informed that he would be playing an interactive game with two addressees in turns; either an adult or a child ('5-year-old'). They were told that the game partners were sitting in other rooms and that they could see the bird token and the digital game board on their monitors. In fact, the confederate performed the role of both addressees, while remaining blind to which one of the two roles he was performing in any given trial. At the onset of each trial the confederate did not know the location of the acorn, and he could infer that location only from the movements of the participant. There were a total of 50 trials, and the participant alternately interacted with the two pseudo addressees in blocks of five trials ( $\sim 35$ min, Figure $2 \mathrm{~B})$. There were two presentation orders of adult-child addressees and two sets of target configurations, counterbalanced over participants. There were two confederates distributed across participants. The game was programmed using Presentation (Neurobehavioral Systems, Albany, CA, USA) software on a Windows XP personal computer.

\section{Data analysis}

Participants' behaviors were analyzed offline, focused on the periods when the participants moved the bird. This study builds on the findings of previous reports involving the same task. Those findings were obtained in a group of women (Newman-Norlund, et al., 2009) and in a group of five-year-olds (Stolk, Hunnius, et al., 2013), showing that the Communicators' beliefs about the age of the Addressees changed their communicative behavior. More precisely, the participants in those studies spent longer time on communicatively relevant locations of the game board when interacting with a presumed young Addressee (vs. an older Addressee), i.e. using time as a tool to place emphasis on the location of the acorn ('Target'). Assuming that we could replicate those findings in the healthy and lesion control groups, this study was designed to test whether a focal vmPFC lesion interferes with the ability of those patients to adjust their communicative behavior to the presumed abilities of the Addressee. Accordingly, we considered the same dependent measure used in previous studies, namely the time spent on game board locations, calculated as the time interval between the first contact of the finger on the touch screen within the area of a square of the game board and the subsequent contact of the finger within the area of a neighboring square of the game board. We distinguished between Target (square containing the acorn) and Non-target locations (other visited locations on the game board) and considered the mean time spent on those location types per trial. It should be emphasized that, given the absence of temporal restrictions on the total time the participant could spend on the game board, the time spent on Target locations and the time spent on Non-target locations could vary independently. We also screened for potential outliers 
(indicative of procedural uncertainties), considering an observation an outlier when the probability associated with either mean time spent on Target or on Non-target location was $1 \%$ or less ( $\mid Z$-score $\mid>\sim 2.5$ ). We also checked for multivariate outliers using pairwise distances (Mahalanobis' distance) between these measures, employing a 5\% probability threshold as an outlier criterion, leaving $89.3 \pm 2.5 \%$ (mean \pm SD) of the original trials for further analysis ( 45 trials).

The main analysis tested for between-groups differences in communicative adjustment to the age of the Addressee, using a univariate ANOVA with Group (vmPFC, LC, HC) as between-subjects factor, and the degree of communicative adjustment in each participant as dependent variable. Communicative adjustment was indexed as the relative difference in time spent on Target locations between presumed child and adult Addressee ([child - adult] / [adult]). This withinsubject normalization procedure reduces the influence of between-subjects variance due to individual differences in (unrestricted) movement times. The sources of the between group differences in communicative adjustments were further qualified with post-hoc comparisons using Fisher's least significant difference (LSD) and paired t-tests, as appropriate. Furthermore, we tested whether the within-group communicative adjustments were communicatively specific, using a two-way repeated-measures ANOVA with Addressee (Adult, Child) and Location (Target, Non-target) as factors. This analysis directly contrasts the between-addressee difference in time spent on a communicatively relevant location of the game board (Target location) with the betweenaddressee difference in time spent on other locations of the game board (Nontarget locations) (see also (Newman-Norlund, et al., 2009; Stolk, Hunnius, et al., 2013).

Two control analyses were used to assess the specificity of the communicative adjustments. First, we tested whether the between-group differences in communicative adjustments were driven by generic motor-related differences between groups, using a multivariate ANOVA with a between-subjects factor of Group (vmPFC, LC, HC) and a within-subjects factor of Addressee (Adult, Child). This analysis considered the dependent variables of planning time, movement time, number of moves, movement time of the confederate, and communicative success. Second, we tested whether the between-group differences in communicative adjustments were driven by the vmPFC group being generically slower in their performance than the other groups, using a two-way univariate ANOVAs with factor Group (vmPFC, LC, HC) and Locations (Target, Non-target). Post-hoc tests (Fisher's LSD) and linear regression analysis were used to further qualify those between-group differences.
A follow-up analysis tested whether vmPFC patients adjust their behavio following a communicative error, better distinguishing the location where the acorn was located from other visited locations of the game board (NewmanNorlund, et al., 2009; Stolk, Hunnius, et al., 2013). To measure the degree of adjustment, we used the 'discriminability' between those locations types, calculated per trial as the mean time spent on target locations divided by the mean time spent on non-target locations (Blokpoel, et al., 2012). Of interest is how the discriminability changes after a communicative error has occurred, indexed as the relative difference in discriminability between the trial following a communication error and the preceding trial that resulted in that error ([trial ${ }^{\mathrm{t}}$ trial $\left.\left.^{t-1}\right] /\left[\operatorname{trial}^{\mathrm{t}-1}\right]\right)$. These adjustments only involved $\mathrm{t}-1$ trials that were preceded by successful communication, i.e. trials that were not post error adjustments themselves. We then considered the mean change in discriminability for each participant and tested between group differences in post communication error adjustment using Wilcoxon rank sum tests for independent samples.

\section{Voxel-based lesion-adjustment mapping}

To assess the fine-scale anatomical relationship between lesion location and communicative adjustment to the mental model of an addressee, we mapped lesion-adjustment patterns using 'voxel-based lesion-symptom mapping' (VLSM; (Bates, et al., 2003; Rorden \& Karnath, 2004)). This method does not require participants to be grouped by lesion site as in the analysis described above, but instead makes use of continuous lesion information, assessing the relevance of each voxel, whether involving white or grey matter, for making communicative adjustments. For each voxel, participants from the vmPFC, LC, or HC groups that did not have a lesion in that voxel were selected and the distribution of communicative adjustments made by these participants were tested agains null, i.e. no communicative adjustment, using a one-sample t-test. Because each voxel used a t-test with varying degrees of freedom, the resulting t-scores were converted to Z-scores of an equivalent probability distribution. 


\section{Results}

\section{Communicative success}

The percentage of successfully communicated trials across participants was 59 $\pm 20 \%$ (mean \pm SD). This is well above chance level $(6.7 \%$; 15 potential target locations). The vmPFC patient group solved $66 \pm 12 \%$ of the trials, the lesion control group (LC) $55 \pm 25 \%$, and the healthy control group (HC) solved 59 $\pm 21 \%$. There were no statistically significant differences in communicative success between the different participants groups, nor as a function of presumed addressee (see below)

\section{Communicative adjustments to the presumed abilities of an interlocutor}

We tested whether patients with a lesion in ventromedial prefrontal cortex are able to adapt their referential communicative behavior to the presumed cognitive abilities of their interlocutor, as inferred from the interlocutor's age (Figure 2B). A univariate ANOVA with a between-subjects factor of Group (vmPFC, LC, HC) was used to compare the different sample populations on the degree of communicative adjustment to the beliefs of an interlocutor, indexed by the relative time spent on Target locations - where the acorn was located - between presumed child and adult Addressee. There was a main effect of participant group on communicative adjustment, $F(2,28)=3.4, p=.046$, effect size partial $2=.20$. Post-hoc analysis (Fisher's LSD test) indicated that this effect was attributable to the group of patients with ventromedial prefrontal cortex (vmPFC) lesions, showing statistically significant differences with the lesion control (LC) group, $p=.035$, and the healthy control (HC) group, $p=$ .023 , see Figure 3A. There were no statistically significant differences between the lesion and healthy control group (LC vs. HC), $p=.91$.

We then assessed whether those adjustments were specific to communicatively relevant locations of the game board. Three repeated measures ANOVAs, separately for each participant group (vmPFC, LC, HC), were used to test for statistically significant interaction effects of the factors Addressee (Adult, Child) and Location (Target, Non-target) on the mean time spent on game board locations. All groups showed a main effect of Location, vmPFC: $F(1,7)=10.8, p$ $=.013$; LC: $F(1,7)=18.6, p=.004$; HC: $F(1,7)=7.5, p=.016$, indicating that participants in this study spent longer on communicatively relevant locations than other visited locations of the game board. However, statistically significant interaction effects between the factors Addressee and Location were confined to the LC and HC groups, $F(1,14)=5.2, p=.038$ and $F(1,7)=14.2, p=.007$

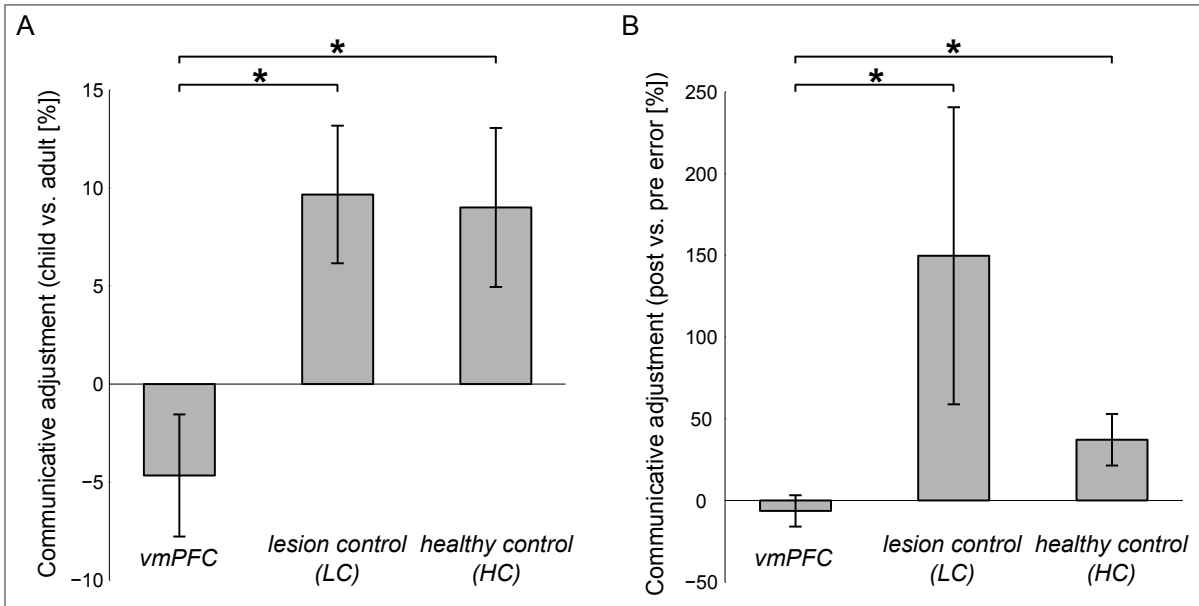

Figure 3. Communicative adjustments made by the participants while moving the bird across the game board, separately per participant group (vmPFC, LC, and HC). (A) Communicative adjustment was indexed as the relative difference of time spent on the Target location - where the acorn was located - between presumed child and adult Addressees ([child - adult] / [adult]). (B) Communicative adjustment was here indexed as the relative difference in 'discriminability' (of the acorn's location from other visited locations) between the trial following a communication error and the preceding trial that resulted in that error $\left(\left[\right.\right.$ trial $^{t}-$ trial $\left.^{t-1}\right] /\left[\right.$ trial $\left.\left.^{t-1}\right]\right)$. Asterisks denote $p<.05$. Error bars denote SEMs.

respectively (vmPFC: $p=.12$ ). In the LC group, there was also a main effect of Addressee on time spent at locations of the game board, $F(1,7)=8.4, p=$ .023 (vmPFC: $p=.10$; HC: $p=.12$ ), likely driven by strong communicative adjustments on Target locations.

Further investigation of the above mentioned interaction effects, using twosided pairwise t-tests, showed that participants in the LC and HC group spent more time on the Target locations (containing the acorn) when they thought to be interacting with the child Addressee as compared to the adult Addressee, $t(7)=3.5, p=.010$ and $t(14)=2.6, p=.022$ respectively. This communicative adjustment, in mean time spent on Target locations, was absent in the vmPFC group, $p=.11$, see Figure 4. Furthermore, none of the participant groups showed statistically significant differences between the two Addressee types for the mean time spent on Non-target locations (other visited locations), all groups $p>.19$. 


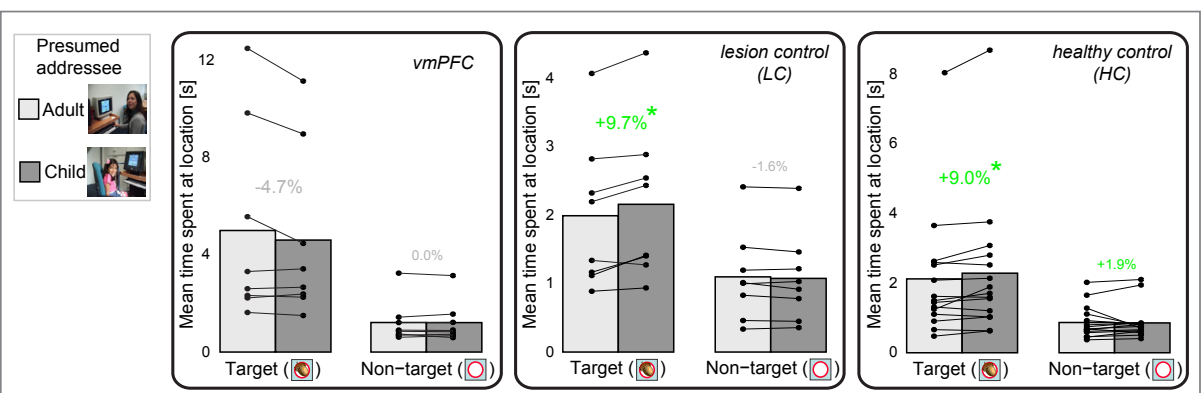

Figure 4. Time spent on Target and Non-target locations (average time per trial) by the participants as a function of presumed adult and child Addressee, separately per participant group (vmPFC, LC, and HC). Similarly to the lesion control (LC) and healthy control (HC) groups, patients with damage to the ventromedial prefrontal cortex (vmPFC) spent longer on communicatively relevant (Target) than irrelevant locations (Non-target). Unlike the lesion and healthy control groups, mean time spent on Target locations by the vmPFC group did not differ between presumed adult and child Addressees. The colored numbers indicate the adjustments made per location type by the participants (average over the group), indexed as the relative difference of time spent on the type of location between presumed child and adult Addressees. Asterisks denote $p<.05$, as determined by pairwise t-tests.

Control analysis \#1: communicative vs. generic motor effects A multivariate ANOVA with a between-subjects factor of Group (vmPFC, LC, HC) and a within-subjects factor of Addressee (Adult, Child) was used to investigate whether these factors had an unexpected influence on other dependent measures of task behaviors. We found a main effect of Group on the number of moves made by the participants during the movement interval, $F(2,62)=4.5, p=.015$, and on response times of the confederate, $F(2,62)=$ $4.8, p=.012$. Post-hoc analysis (Fisher's LSD test) showed that the main effect of Group on number of moves made by the participants pertained to the vmPFC patients making fewer movements than the healthy control group (HC), $p=$ .004 (vmPFC vs. LC, $p=.16$; LC vs. HC, $p=.18$ ). The main effect of Group on response time of the confederate was attributable to faster confederate response times in the lesion control group (LC) than in the vmPFC and healthy control group (HC), $p=.004$ and $p=.028$ respectively (vmPFC vs. HC, $p=.24$ ), likely originating from the fact that most of the patients from the lesion control group interacted with a different confederate than the other sample populations. Given that communicative adjustments were observed in interactions involving both confederates, i.e. within the LC and HC groups, it is unlikely that confederate response times could have differentially influenced communicative adjustments in the vmPFC group, given that this group interacted with the same confederate as the HC group. There were no main effects of Group on participants' planning times, movement times, or communicative success, all $p>.24$. There were no main effects of Addressee or interaction effects of Group and Addressee on any of the dependent variables of planning time, movement time, number of moves, confederate response time, or communicative success, all $p>.50$.

\section{Control analysis \#2: Target vs. Non-target locations}

We also tested whether vmPFC patients spent relatively longer on any of the game board location types than the other groups. A univariate ANOVA with a between-subjects factor of Group (vmPFC, LC, HC) and a within-subjects factor of Location (Target, Non-target) was used to investigate for the influence of these factors on the mean time spent at a game board location. There was a main effect of Group, $F(2,56)=4.1, p=.021$, and a main effect of Location, $F(1,56)=18.1, p<.001$, with the latter indicating that participants spent longer on communicatively relevant than irrelevant portions of the game (cf. mean time spent on Target vs. Non-target of each group, Figure 4). An interaction effect of Group and Location did not reach statistical significance, $F(2,56)=$ $2.8, p=.070$. Post-hoc analysis (Fisher's LSD test) of the main effect of Group on mean time spent at locations of the game board indicated that this effect was attributable to the group of patients with ventromedial prefrontal cortex (vmPFC) lesions, spending significantly longer on portions of the game board than the lesion control (LC) and healthy control group, $p=.025$ and $p=.009$ respectively (LC vs. HC, $p=.93$ ). Further exploration of this effect using two univariate ANOVAs with factor Group (vmPFC, LC, HC) showed that there was an effect of Group on time spent on Target locations, $F(2,28)=3.6, p=.039$ but not on Non-target locations, $p=.43$. Post-hoc analysis of this main effect of Group on time spent on Target locations indicated that the vmPFC group spent longer on Target locations than the other groups, vmPFC vs. LC, $p=.030$; vmPFC vs. HC, $p=.019$ (LC vs. HC, $p=.90$ )

We next tested whether the lack of communicative adjustment to the presumed cognitive level of an addressee in the vmPFC group could be a consequence of this sample population spending relatively longer on the Target locations of the game board. A linear regression analysis was used to assess the relationship between a vmPFC patient's mean time spent on target location and the same patient's communicative adjustment to a presumed addressee. There was no statistically significant relation between these two dependent variables, $p=$ .21 (neither for the other groups; LC, $p=.61$; HC, $p=.86$ ), indicating that the magnitude of communicative adjustment was not a function of mean time spent on communicatively relevant locations. 


\section{Lesion-adjustment mapping}

To confirm the above findings, and to gain more insight in which parts of ventromedial prefrontal cortex are particularly relevant for making communicative adjustments, we mapped lesion-adjustment patterns on a voxel-by-voxel basis. For each brain voxel, participants from the vmPFC, LC, or HC groups that were spared from a lesion in that voxel were selected and the communicative adjustments made by these participants were tested against a null distribution of no communicative adjustment. In other words, this analysis was not based on a-priori categorization of the participants in an experimental group, nor restricted to the vmPFC region. The results indicate that excluding patients with a lesion to a region of the right ventromedial prefrontal cortex yielded largest Z-scores, i.e. consistently larger communicative adjustments over the group (Figure 5A). The lack of adjustment at Non-target location (Figure 5B) indicates that these adjustments were confined to communicatively relevant locations of the game board (Target locations).
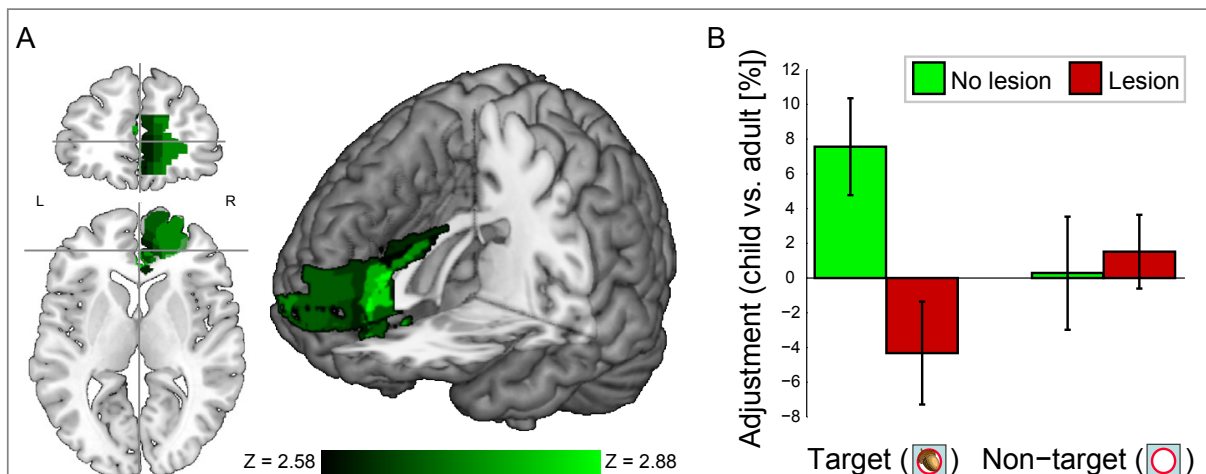

Figure 5. (A) Voxel-based lesion-adjustment mapping showing voxel-wise relevance for making communicative adjustments between presumed child and adult Addressees. For each voxel, participants spared from a lesion to that voxel were selected and the communicative adjustments by these participants were compared against a null distribution of no adjustment. The threshold of the color axis was adjusted to resolve the spatial structure around the statistically significant peaks: Z-scores correspond to $.005>p>.002$. (B) Adjustments as a function of voxel status (No lesion, Lesion) and board location (Target, Non-target), for a peak voxel (MNI coordinates $[1,46,5]$, indicated by crosshairs in panel A). Error bars denote SEMs.

\section{Communicative adjustments following communicative errors}

All participant groups spent longer on communicatively relevant than on irrelevant locations of the game board, distinguishing the location where the acorn was located from other visited locations of the game board. We also tested whether vmPFC patients adapt this type of behavior following a communicative error. To measure the degree of adjustment, we used the 'discriminability' between those locations types, calculated per trial as the mean time spent on target locations divided by the mean time spent on non-target locations (Blokpoel, et al., 2012). Of interest is how the discriminability changes after a communicative error has occurred, indexed as the relative difference in discriminability between the trial following a communication error and the preceding trial that resulted in that error ([trial ${ }^{t}-$ trial $\left.^{t-1}\right] /\left[\right.$ trial $\left.\left.^{\mathrm{t}-1}\right]\right)$. We then considered the mean change in discriminability for each participant and tested the hypothesis that following a lesion in ventromedial prefrontal cortex these post error adjustments are altered. Wilcoxon rank sum tests for independent samples indicated that the vmPFC patient group showed significantly less adjustment following a communication error than both the lesion control group, $p<.001$, and the healthy control group, $p<.043$, see Figure 3B. A wilcoxon signed rank test, testing post error adjustment of the vmPFC lesion group against null, indicated that vmPFC patients did not adjust their communicative behaviors following a communication error, $p>.84$ (LC: $p=.063$; HC: $p=.022$ ). There were no statistically significant differences in discriminability changes between the different groups following a successfully accomplished trial (vmPFC: $12 \pm$ 48\%; LC: $-6 \pm 56 \%$; HC: $5 \pm 36 \%$; mean \pm SD), both $p>.19$. 


\section{Discussion}

In the present study we have tested whether patients with a lesion in ventromedial prefrontal cortex (vmPFC) adapt their referential communicative behavior to the presumed cognitive abilities of their interlocutor, as inferred from the interlocutor's age. Participants were asked to influence the behavior of an addressee, in an experimental setting where the communicative means made available to the participants were purportedly limited, challenging them to devise new communicative behaviors that could be understood by the addressees. We compared vmPFC patients' communicative behaviors evoked during task performance with behaviors of a lesion control group (LC), with extent-matched lesions outside the vmPFC, and with behaviors of a healthy control group (HC), matched on gender, age, and education. There are three main results. First, similarly to lesion and healthy controls, patients with damage to the ventromedial prefrontal cortex spent longer on the location where the acorn was located than on other visited locations of the game board. This finding indicates that a lesion in vmPFC does not alter the ability to produce communicative actions, distinguishing communicatively relevant from irrelevant locations of the game board. Second, in contrast to lesion and healthy controls, patients with vmPFC lesions did not spontaneously spend longer on these communicatively relevant locations when they thought to be interacting with the child addressee. Third, following a communicative error, patients with vmPFC did not increase the contrast between communicative and instrumental components of their actions, as spontaneously done by the LC and HC groups. The latter two observations indicate that vmPFC lesions alter the ability to make referential communicative adjustments to the inferred knowledge of a communicative partner. Those findings suggest a cerebral dissociation between selecting an effective communicative action and adjusting the manner of that action to the presumed characteristics of an addressee.

\section{Communicative decisions in vmPFC patients}

Patients with damage to the ventromedial prefrontal cortex showed no deficit in the overall effect of their communicative actions, with matched performance across groups. They also used strategies similar to those of the control groups, using time as a tool to put emphasis on communicatively relevant locations of the game board. In fact, the present results indicate that they spent even longer on these communicatively relevant locations than the other sample populations, whilst the mean time spent on other visited locations of the game board was matched to that of controls. This finding indicates that a lesion in vmPFC does not interfere with the patients' motivation to communicate (ShamayTsoory, Aharon-Peretz, \& Perry, 2009). The finding is also unlikely to reflect perseveration, a behavior often attributed to vmPFC patients (Bechara, et al., 1994). Those patients did not have problems switching between different task components, as when spontaneously spending more time on communicatively relevant than irrelevant locations of the game board, or switching between reaching a communicatively relevant location and returning to the starting location. It could be argued that longer times spent at communicatively relevant locations by the vmPFC group could have obscured numerically small communicative adjustments made by this sample population. However, the magnitude of the communicative adjustments was not a linear function of time spent on communicatively relevant locations. In fact, the longer times spent on target locations by the vmPFC group are more consistent with the idea that these patients were not able to estimate how much time would be sufficient for their addressees to understand the communicative relevance of that location. First, whereas the LC and HC groups fine-tuned their communicative behaviors to knowledge and beliefs about their addressees, the vmPFC patients disproportionally enhanced the contrast between the time spent on target and on non-target locations, irrespectively of the presumed characteristics of the addressee. Second, the vmPFC patients were not able to dynamically update their mental model of the addressee: following a communicative error, those patients did not increase the contrast between communicative and instrumental components of their actions, as LC and HC did. This effect was not a consequence of failing to detect the occurrence of a communicative error: vmPFC patients moved slower after an error than after a correct trial $(t(7)=2.5, p=.041)$. Taken together, these observations suggest that vmPFC patients' communicative decisions are not informed by a mental model of their communicative partner.

\section{Use and retrieval of social information}

Fine-grained analysis of the anatomical relationship between lesion location and communicative adjustments revealed that the right vmPFC region is particularly important for implementing communicative adjustments. This observation fits with the notion that right-sided vmPFC patients have been found to have profound disturbances within social conduct, decision making, and emotional processing, whereas left-sided vmPFC patients seemed less affected (Tranel, Bechara, \& Denburg, 2002). This finding also fits with recent electrophysiological observations in healthy subjects engaged in a variant of the task used in this study, showing a right hemisphere dominance when participants planned communicative actions (Stolk, Verhagen, et al., 2013). More generally, grey matter density in the vmPFC has also been linked to the size of the social network and status of an individual (Lewis, Rezaie, Brown, Roberts, \& Dunbar, 2011; Sallet et al., 2011), in line with a role of this region in processing a menta model of the social world. Yet, the vmPFC seems to be particularly geared 
towards using social knowledge for biasing current decisions, rather than verbal retrieval. In contrast to the lack of communicative adjustments observed during task performance, the vmPFC patients accurately rated their addressees' ages when probed. This dissociation between use and verbal retrieval of social information in vmPFC patients fits with previous observations, showing that those patients are unaware of their socially inappropriate behavior during conversations, but not when seeing it on video (Beer, et al., 2006). Furthermore, vmPFC patients' concepts of gender are still intact and can be explicitly probed, but this knowledge is not automatically taken into account during a response task (Milne \& Grafman, 2001). It is conceivable that previous reports describing un-impaired attribution of mental states to other agents (Bird, Castelli, Malik, Frith, \& Husain, 2004), or un-impaired audience design effects during verbal interactions (Gupta, et al., 2012) stem from the apparently preserved ability of patients with vmPFC lesions to effectively retrieve verbal knowledge on social facts and procedures.

\section{Conclusion}

This study exploits an unfortunate experiment of nature to test whether a lesion in the ventromedial prefrontal cortex impairs human ability to adapt their referential communicative behavior to a mental model of their communicative partner. Specificity of the effects of brain damage is ensured by lesion-symptom mapping and by comparison with communicative behaviors evoked by lesion and healthy control subjects. Following a lesion to the ventromedial prefrontal cortex, patients were still motivated and able to select and alter effective communicative actions but failed to spontaneously adjust their communicative behavior to the addressee's performance and presumed abilities. These findings qualify the contributions of the ventromedial prefrontal cortex to human communicative abilities, showing that this region might be necessary for informing communicative decisions with inferred knowledge of a communicative partner. 

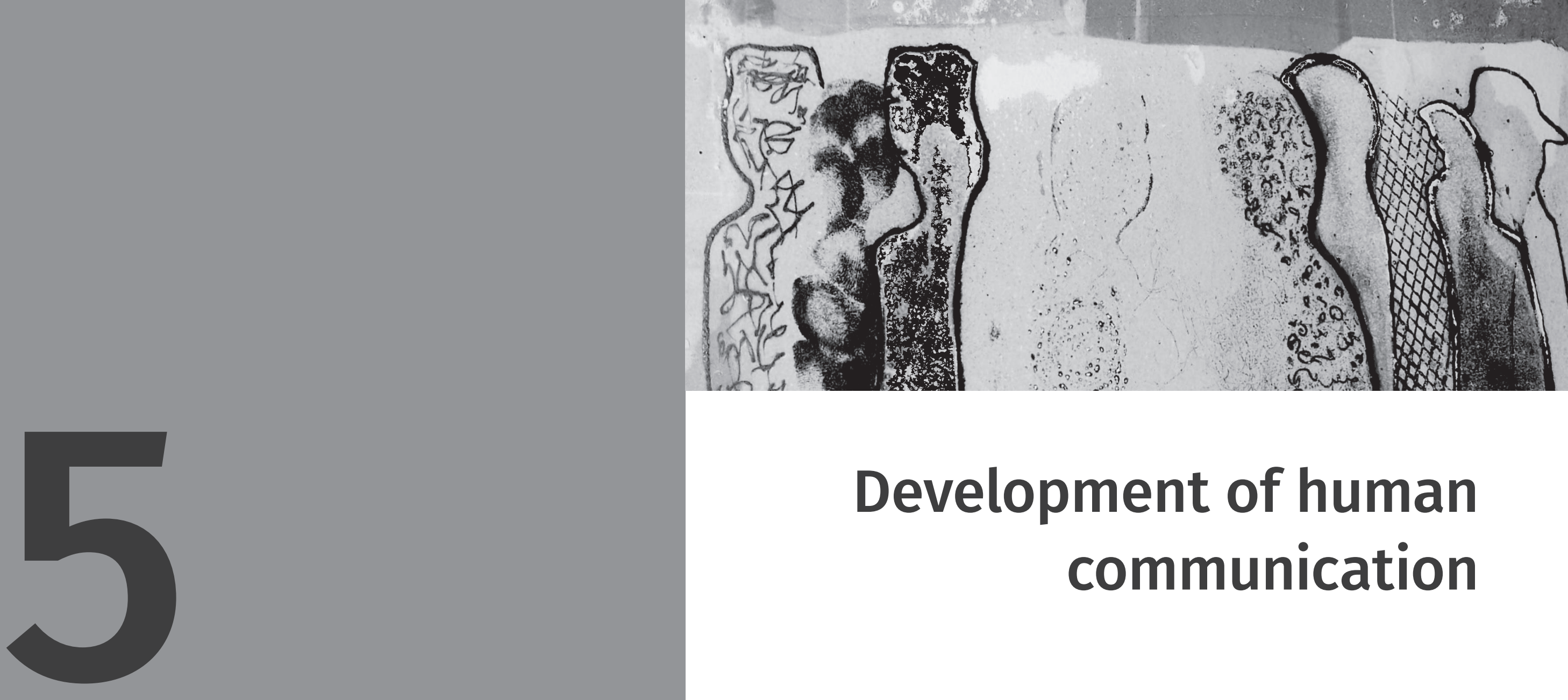


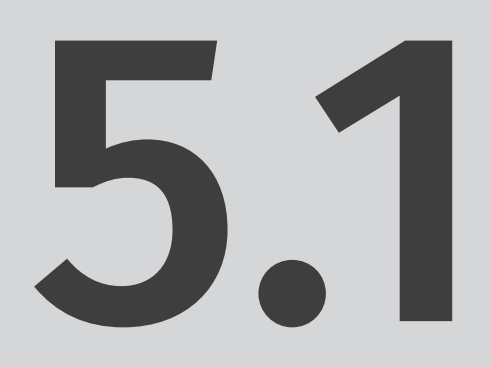

Early social experience predicts referential communicative adjustments in fiveyear-old children

Stolk A, Hunnius S, Bekkering H, Toni I 


\section{Abstract}

A large body of work has focused on children's ability to attribute mental states to other people, and whether these abilities are influenced by the extent and nature of children's social interactions. However, it remains largely unknown which developmental factors shape children's ability to influence the mental states of others. Building on the suggestion that collaborative experiences early in life might be crucial for the emergence of mental coordination abilities, here we assess the relative contribution of social exposure to familial and non-familial agents on children's communicative adjustments to their mental model of an addressee ('audience design'). During an online interactive game, five-year-olds spontaneously organized their non-verbal communicative behaviors according to their beliefs about an interlocutor. The magnitude of these communicative adjustments was predicted by the time spent at daycare, from birth until four years of age, over and above effects of familial social environment. These results suggest that the degree of non-familial social interaction early in life modulates the influence that children's beliefs have on their referential communicative behavior.

\section{Introduction}

Humans often use un-observable variables like beliefs, desires, and intentions to disambiguate agents' behavior, attributing mental states to other people and to oneself (Frith \& Frith, 2006; Premack \& Woodruff, 1978). These mentalizing abilities emerge during early childhood (Frith \& Frith, 2003) and variations in mentalizing skills appear to be related to social environmental factors (Ronald, Viding, Happe, \& Plomin, 2006). Among these factors, collaborative experiences of a child with adult group members might play a crucial role (de Rosnay \& Hughes, 2006; Hrdy, 2009). These interactions might allow children to gradually construct knowledge of the world, as well as knowledge of other people's mental states, by capturing cognitive regularities that cooperative agents try to make transparent to the child (Carpendale \& Lewis, 2004). Eventually, children start using this knowledge to manipulate the mental states of other agents during referential communicative interactions. For instance, 4-year-old children use presumed knowledge of an interlocutor to select linguistic behaviors designed to change those mental states, producing more explicit descriptions of a toy when speaking to a blind as compared to a non-blind addressee (Maratsos, 1973), and simpler utterances towards a toddler than an adult (Shatz \& Gelman, 1973). Five-year-old children can produce verbal requests that take into account the presumed knowledge of their interlocutor (Nilsen \& Graham, 2009). However, it remains largely unknown how children learn to adjust their referential communicative behaviors to their mental model of an addressee.

Here we elaborate on the suggestion that the extent and nature of the social interaction children experience will influence the development of children's social understanding (Carpendale \& Lewis, 2004; Cole \& Mitchell, 2000; de Rosnay \& Hughes, 2006; Lewis, Freeman, Kyriakidou, Maridaki-Kassotaki, \& Berridge, 1996; Perner, Ruffman, \& Leekam, 1994). Humans are exceptional among existing hominids for experiencing early developmental exposure to cooperative nonkin (Burkart, Hrdy, \& Van Schaik, 2009), i.e. conspecifics that lack a genetic reason for collaborating, and it has been suggested that this developmental feature might boost motivational predispositions to share mental states with others (Hrdy, 2009). We quantify one aspect of this faculty through audience design, i.e. adjustments of communicative acts to the presumed abilities and knowledge of an interlocutor (Clark, 1996). Given that audience design presupposes control of the ability to share mental states with others, we focus on five-year-old children, i.e. children with fully-fledged theory of mind capacities (Wellman, Cross, \& Watson, 2001). We quantify developmental exposure to two main sources of social interactions experienced by children between zero and four years of age, namely familial and non-familia 
experiences. The former were quantified in terms of years of experience with siblings, and parents' level of education. The latter were quantified in terms of days per week of attendance to daycare (Cole \& Mitchell, 2000; Cutting \& Dunn, 1999; Holmes, Black, \& Miller, 1996; Perner, et al., 1994; Ruffman, Perner, Naito, Parkin, \& Clements, 1998).

Audience design effects were quantified in a controlled experimental setting involving the production of referential non-verbal behaviors with a communicative goal (de Ruiter, et al., 2010), exploiting a protocol previously validated in adults (Newman-Norlund, et al., 2009). In contrast to linguistic communication, the communicative behaviors evoked under these experimental conditions could not be directly based on previous concrete experiences. Accordingly, the novel communicative situation experienced by the children in this study allowed us to directly tap into their ability to influence the mental states of others through behaviors generated ex-novo. Five-year-old participants were told they were playing an online interactive game with a 2-year-old toddler and with a same-age peer, in alternation. In fact, a confederate performed the role of both addressees, while remaining blind to which one of the two roles he was performing in any given trial. Accordingly, both performance and response times of the two presumed addressees were matched. This feature of the protocol allowed us to test whether the mere belief that the child is communicating with addressees of different ability induces internally generated adjustments in the child behavior, over and above performance-related mutual adjustments (Hasson, et al., 2012; Pickering \& Garrod, 2007). Furthermore, the precise quantification of children behavior afforded by this protocol distinguished between belief-driven adjustments restricted to the communicative components of the actions, and generic priming effects (Bargh, Chen, \& Burrows, 1996; Marshall, Bouquet, Thomas, \& Shipley, 2010). These procedures allowed us to test whether the social environment experienced by a child early during his development influences his ability to adjust a self-generated communicative behavior to his mental model of the addressee.

\section{Materials and Methods}

\section{Participants}

The experiment was approved by the local medical ethical committee (ECG, Nijmegen, The Netherlands). Parents with 5 -year-old children $(\mathrm{N}=24,12$ females, mean age 5.09, range 5.02 - 5.16) were recruited from a database of the Baby Research Center Nijmegen. The children's parents provided written informed consent for participation of their children in the study, and all participants received a book or monetary compensation for their visit.

\section{Experimental design}

The game involves a Communicator (a 5-year-old participant, displayed as a bird on the game board) and an Addressee (the confederate, displayed as a squirrel) interacting on a digital game board with a $3 \times 3$ grid layout (see Figure 1A). On each trial, their joint goal was for the Addressee to collect an acorn from the game board. Given that knowledge of the acorn's location in the game board was available to the Communicator only (on a printed copy of the game board, visible throughout the trial, see Figure 1A), a successful trial of this game required the Communicator to inform the Addressee where the acorn was located. Given the experimental setup, the Communicator could inform the Addressee only by moving the bird across the game board (event 2 in Figure 1B). The Addressee could then move the squirrel to the acorn's location only by interpreting the meaning of the Communicator's movements on the game board (event 3 in Figure 1B). For details on the experimental procedure see Appendix A of this chapter.

By touching a square on the screen with his/her finger, the Communicator could move the bird token to that square, and this movement was also visible to the Addressee. The bird could only move to the center of each of the nine grid squares, and only through vertical or horizontal displacements. This feature of the task was introduced to create a spatial disparity between the movements of the bird and the potential locations of the target object (any of the thirteen white circles, see Figure 1B). Namely, the bird could not be overlaid on the precise location of the acorn when a square contained more than one white circle (see Manipulation of task difficulty of Appendix A for details). The Communicator had no restrictions on planning time (event 1 in Figure 1B) or on movement time (event 2). The end of the movement epoch was marked by the return of the bird on the central square of the game board (nest). At this point, the token of the Addressee (the squirrel) appeared, in the center of the digita game board, visible to both players. The Addressee moved the squirrel to the location deemed appropriate given the movements of the Communicator (event 
3). The Addressee had no temporal or spatial restrictions on the movements of the squirrel on the game board. Successful trials, in which the Addressee had moved to the location of the target, resulted in the presentation of a large acorn on the screen (event 4). A red "no" icon was presented over a small acorn for unsuccessful trials.

There were a total of 50 trials, subdivided in blocks of five trials $(\sim 35 \mathrm{~min}$, Figure 1C). Each child was informed that he would be playing an interactive game with two addressees in turns; either a toddler ('2-year-old') or a sameage peer ('5-year-old'). They were told that the game partners were sitting in other rooms and that they could see the bird token and the digital game board on their monitors. There were two pairs of fictitious child-toddler addressees, two presentation orders of child-toddler addressees, and two sets of target configurations, counterbalanced over participants.

\section{Quantification of the social environment}

Given that the extent and nature of the social interactions experienced by children is widely thought to influence the development of their social understanding (Carpendale \& Lewis, 2004; de Rosnay \& Hughes, 2006; Lewis, et al., 1996; Perner, et al., 1994), we considered two main sources of social interactions experienced by children, namely familial and non-familial experiences, reconstructed from interviews with the parents of the children. Familial experiences were indexed with the parents' level of education (11 levels, $7.4 \pm 1.6$, group mean $\pm \mathrm{SD}$, range $4.5-10.5$ ) and years of experience with siblings (i.e. the product of age and number of siblings: $4.3 \pm 3.4$, range 0 - 15.2; number of siblings: $1.2 \pm 0.7$, range $0-3$ ). Non-familial experiences were indexed with the time spent at daycare (days per week) between the age of 0 and 4 (mean over these four years; $1.7 \pm 0.9$ days per week, range $0.25-3$ ) We did not consider between ages 4 and 5 given that in the Netherlands it is customary to start primary school at age 4 .

\section{Data analysis}

Audio- and video-recordings of the participant's behavior were analyzed offline. Those trials in which the child behavior revealed procedural uncertainties (e.g. failing to return to the nest within 15 seconds, or interrupting the bird movements to look at the location of the acorn in the instruction game board) were excluded, leaving $80.1 \pm 13.4 \%$ (mean \pm SD) of the original trials for further analysis ( $\sim 40$ trials; four participants interrupted their performance after 30 trials).
This study builds on the findings of a previous report involving the same task and obtained in a group of women (Newman-Norlund, et al., 2009), showing that the communicator's belief about age of the addressee changed communicative behavior. More precisely, these adults spent longer time on communicatively relevant locations of the game board when interacting with a presumed child addressee (vs. an adult addressee), i.e. using time as a tool to place emphasis on target information. The first goal of this study was to replicate this finding in a
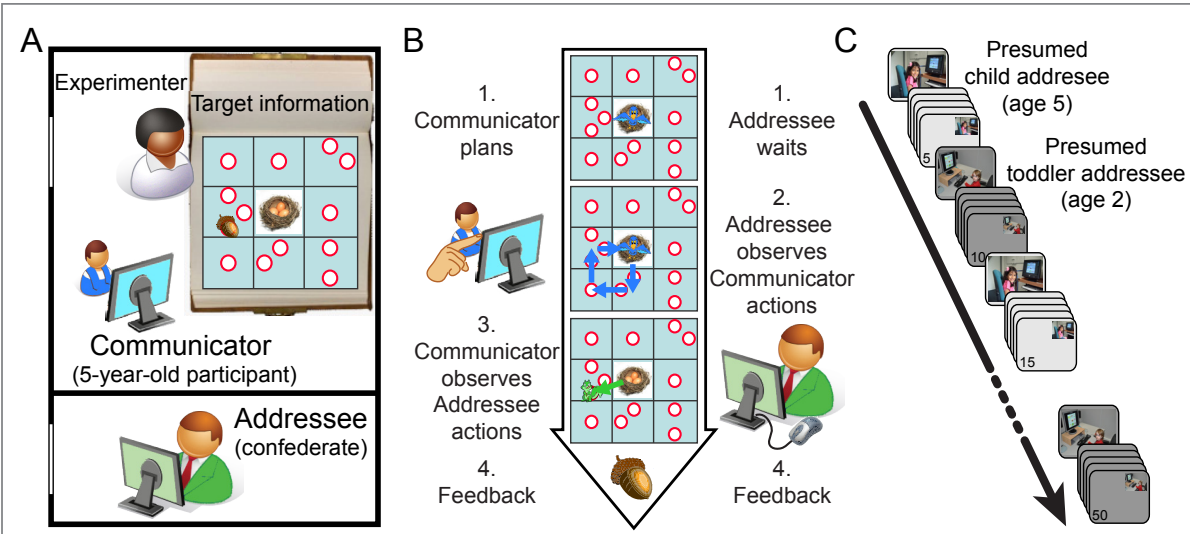

Figure 1. Task setup. (A) The Communicator, a 5-year-old participant, sat next to an Experimenter who provided the task instructions and the trial-specific location of the acorn but played no part in the communicative game. The Addressee, a confederate who performed the role of a toddler and a child (see panel C), while remaining blind to which one of the two roles he was performing in any given trial, sat outside the experimental room facing another monitor. (B) Each single trial encompassed four successive events. (1) the Experimenter showed to the Communicator only the location of the acorn (see panel A), and the Communicator had unlimited time to plan the movements; (2) the Communicator moved the bird icon on the game board by touching a touch-screen with a finger (the movements of the bird were visible to both Communicator and Addressee); (3) the Addressee moved the squirrel icon on the game board with a digital mouse (the movements of the squirrel were visible to both Communicator and Addressee); (4) both players received common feedback on the communicative success of the trial. Note that the bird, unlike the squirrel which could move freely, could only move to the center of each of the nine grid squares, and only through vertical or horizontal displacements. This feature of the task made it difficult for the Communicator and the Addressee to discriminate the location of multiple potential targets within a square (the white circles) on the basis of the location of the bird alone. (C) A digital photograph of the current presumed addressee was presented to the Communicator in full screen before the onset of each block of 5 trials, and in the top right corner during each block. 
group of five-year-old children. Accordingly, we considered the same dependent variable (namely, Time spent on game board locations), using the same statistical comparison, namely a two-way ANOVA with factors Addressee (Toddler, Child) and Location (Target, Non-target). The Time spent on game board location by the Communicator was calculated as the time interval between the first contact of the finger on the touch screen within the area of a square of the game board (either a Target or a Non-target location) and the subsequent contact of the finger within the area of a neighboring square of the game board. We considered the mean time spent on those location types per trial. It should be emphasized that, given the absence of temporal restrictions on the total time the children could spend on the game board, the time spent on target locations and the time spent on non-target locations could vary independently.

Having replicated the findings of (Newman-Norlund, et al., 2009) in this group of five year-olds (Figure 2), we used a multiple linear regression analysis to assess the differential contribution of familial and non-familial sources of social interactions experienced by these children in the first four years of their life. These three independent variables (i.e. parents' level of education, years of experience with siblings, and time spent at daycare, see above) were jointly considered in the multiple regression analysis, with the degree of communicative adjustment observed in each child as dependent variable (i.e. the relative difference, [toddler - child] / [child], in time spent on Target locations between presumed toddler and child Addressee). This statistical approach allows one to make specific inferences on the inter-subject variance accounted for one variable, over and above the variance accounted by the other variables included in the multiple regression model.

\section{Results}

\section{Communicative success}

The percentage of successfully communicated trials was $63.4 \pm 8.0 \%$ (mean \pm $\mathrm{SD})$. This is well above chance level (7.7\%; 13 potential target locations).

\section{Communicative adjustments}

We tested whether 5-year-old children are able to adapt their referential communicative behavior (event 2 in Figure 1B) to the presumed age, or cognitive level, of their interlocutor. A two-way analysis of variance revealed a significant interaction of the factors Addressee (Toddler, Child) and Location (Target, Non-target) on the mean time spent on game board locations during the movement epochs, $F(1,23)=5.4, p=.03$. This interaction was driven by the fact that the 5-year-old children spent more time on the Target locations (containing the acorn) when they thought to be interacting with the toddler Addressee as compared to the child Addressee, $t(23)=2.6, p=.014$, two-sided paired t-test. There was no difference between the two Addressee types for the mean time spent on the Non-target locations (other visited locations), $t(23)=$ $0.04, p=.97$; see Figure 2 .

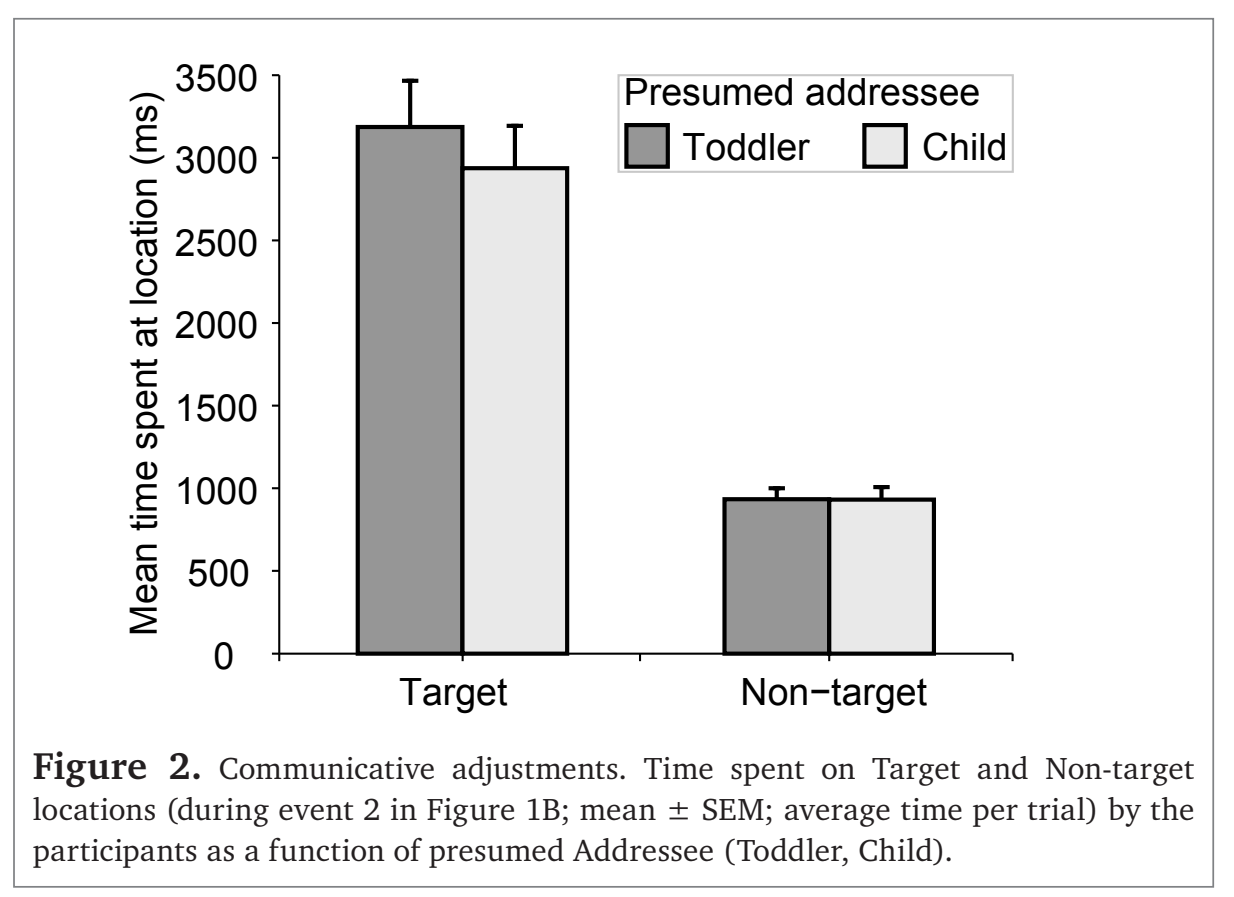




\section{Effects of social environment}

We evaluated whether quantitative indexes of developmental exposure to social interactions of the child could explain inter-individual variability in the communicative adjustment observed over the whole group. A multiple linear regression analysis indicated that daycare attendance (i.e. mean days per week spent at daycare before starting school) predicted the communicative adjustments made by the 5 -year-old participants, $\mathrm{R}^{2}=.34, F(3,23)=3.4, p$ $=.039$ (full model), Beta $=.598, p=.005, \mathrm{R}_{\text {adj }}^{2}=.24$ (daycare attendance); see Figure 3. Parents' level of education (Beta $=-.14, p=.45$ ) and years of experience with siblings (Beta $=.04, p=.84$ ) did not significantly account for inter-subject variance in communicative adjustments.

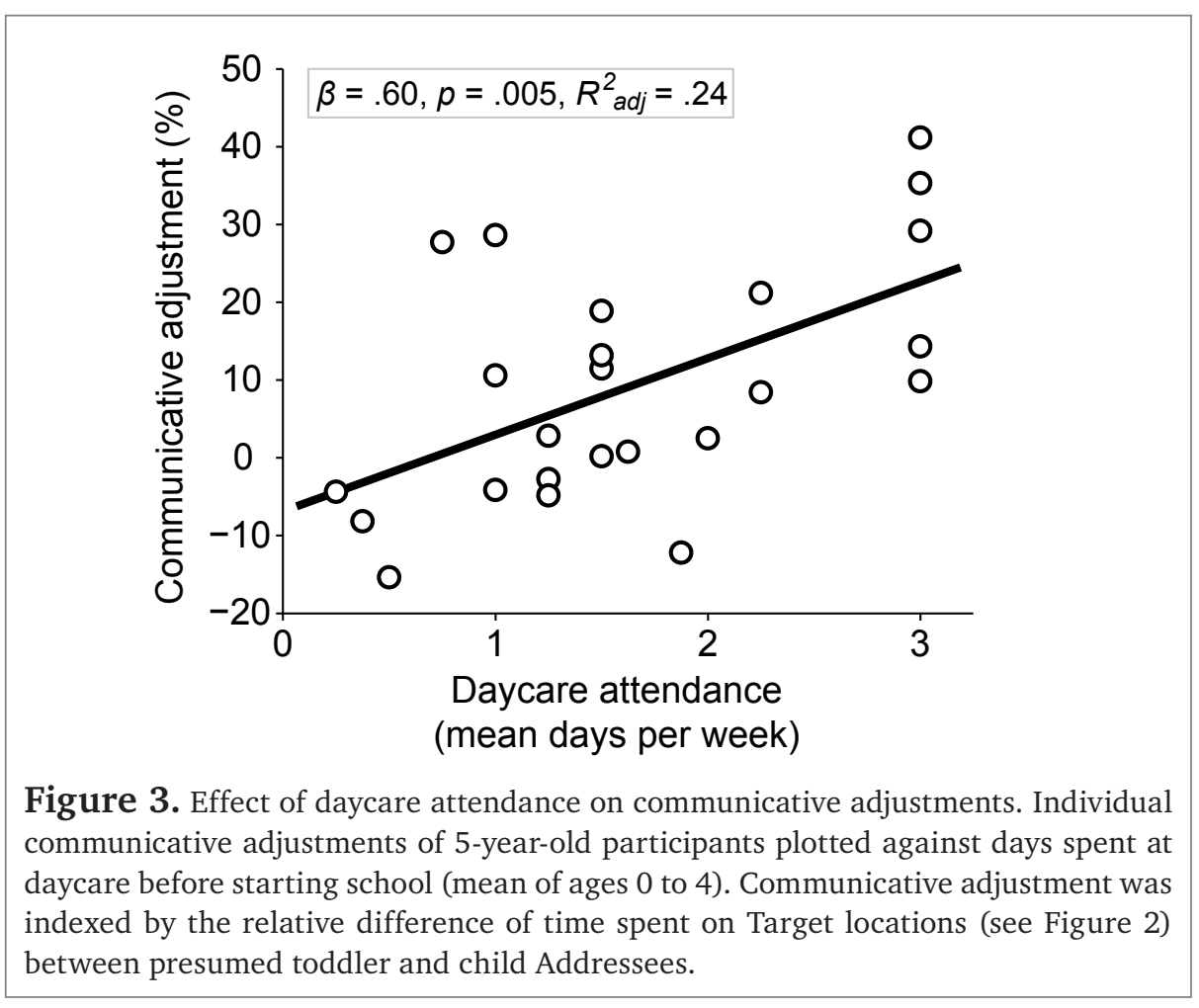

\section{Discussion}

We have tested whether the expression of audience design abilities in 5-year-old children is modulated by their previous history of social interactions. Participants were asked to influence the behavior of an addressee, in an experimental setting where no pre-existing communicative conventions were immediately available. In fact, the communicative means made available to the children were purportedly limited, challenging them to devise new communicative behaviors that could be understood by the addressees. There are three main results. First, 5 -year-old children were able to influence the mental states of others even at their first encounter with a novel communicative setting. This communicative behavior was internally generated by the children, and motorically different from the behavior of the two presumed addressees (Figure 1B). Second, the mere belief of communicating with addressees of different ages selectively influenced the communicative behavior of the participants. The children spent longer at communicatively relevant locations when interacting with a presumed toddler addressee as compared to a presumed child addressee. This communicative adjustment was not a generic priming effect, being absent in communicatively irrelevant locations of the game-board. Third, the communicative adjustment observed in the children was predicted by the time spent at daycare during the previous years of their life. This latter finding refines the notion that human communicative skills might be shaped early during development (Burkart, et al., 2009; Herrmann, Call, Hernandez-Lloreda, Hare, \& Tomasello, 2007), emphasizing the fundamental role of non-familial interactions in the gradual construction of children's social understanding and abilities to influence the mental states of others (Carpendale \& Lewis, 2004; de Rosnay \& Hughes, 2006).

It has been suggested that children gradually construct mental variables through the regularities they experience within social interaction (Carpendale \& Lewis, 2004; de Rosnay \& Hughes, 2006; Peterson \& Siegal, 2000). In contrast to a large body of work focusing on verbal reports of children's ability to attribute mental states to other people, as during Theory of Mind tasks (Baron-Cohen, Leslie, \& Frith, 1985; Wellman, et al., 2001), here we considered children's ability to influence the mental states of others through non-verbal behaviors, i.e. the magnitude of their communicative adjustments. These spontaneous adjustments provided a sensitive index for quantifying inter-individual differences in communicative abilities close to the onset of those abilities. This sensitivity might arise from the implicit nature of the index of audience design used in this study, in line with findings previously obtained during language comprehension in children of similar age (Nilsen \& Graham, 2012; Nilsen, Graham, Smith, \& Chambers, 2008). Namely, in contrast to previous work 
exploring how a child's inhibitory control handles the conflict between the knowledge of the child and that of the addressee (Nilsen \& Graham, 2012), in this study we manipulated the presumed abilities of the addressee, minimizing demands on the control abilities of the child (Rubio-Fernandez \& Geurts, 2013).

The magnitude of communicative adjustments in 5-year-old children was predicted by the time spent in daycare during previous years of their life, over and above the effects accounted for by measures of the familial social environment (sibling experience, educational level of the parents). One possible mechanism accounting for this observation might relate to the importance that overheard communicative interactions have on the linguistic development of a child (Dunn \& Shatz, 1989; Oshima-Takane, 1988; Oshima-Takane, Goodz, \& Derevensky, 1996). Namely, kindergarten attendance might considerably boost the variety of children's experience with this source of pragmatic inputs, enhancing their communicative skills. More generally, the structured social interactions afforded by a daycare environment (e.g. cooperative play, frequent integration of new group members) might provide the child with a larger set of communicative challenges than those experienced within a relatively stereotyped familial environment (Tomasello, 2008). These challenges might differ substantially from those experienced in a familial environment. In kindergarten, a child needs to communicate with a multitude of agents, and those agents lack a genetic reason for collaboration. Finally, kindergarten provides children with caregiving 'alloparents' that might boost their socioemotional development (Van IJzendoorn, Sagi, \& Lambermon, 1992).

It remains to be seen how the present findings, showing stronger effects of non-familial over familial experiences on the development of referential communicative adjustments, can be reconciled with previous reports, showing that measures of familial interactions predicted 'false belief understanding' (Lewis, et al., 1996; Perner, et al., 1994), as assessed with verbal reports. One possibility is that the communicative adjustments observed in this study might be mainly driven by children's assumptions on the presumed cognitive capacities of the addressees, rather than by children's understanding that the beliefs, desires, or intentions of other agents differ from reality (Cole \& Mitchell, 2000; Perner, et al., 1994). Differences in outcome measures might also play a role, e.g. implicit measures of knowledge about a communicative interaction (as gathered through eye movements, reaction time, or movement times) vs. explicit verbal reports requiring a degree of executive control (Nilsen, et al., 2008; Rubio-Fernandez \& Geurts, 2013).
This study opens the way for systematic and sensitive investigations into the contributions of early social experiences towards children's communicative abilities, raising the possibility to chart the developmental trajectories generated by familial and non-familial social interactions (e.g. siblings, parents, nonsibling peers, alloparents) through longitudinal studies with objective measures of the time spent on those interactions. 


\section{Supporting Information}

Experimental procedure

During the experiment, the participant sat in a sound-proof experimental room, facing a monitor displaying the digital game board (Figure 1A), while being video- and audio-recorded. An experimenter sat next to the participant, providing the task instructions and the trial-specific location of the target object (i.e. an acorn), but playing no part in the communicative game. A confederate sat outside the experimental room facing another monitor, showing the same game board seen by the participant. First, each child was familiarized with the experimental setup ( 5 trials, $\sim 5 \mathrm{~min}$ ). During these familiarization trials the child was encouraged to freely move the bird around the game board, experiencing the constraints on the bird's movements as described in the experimental design. The child was also instructed to return the character to the nest (center game board location) at the end of his movements. This task requirement was emphasized by a continuous sound (a bird squeak) that started when the child moved away from the nest and stopped when the child returned the bird to the nest.

Second, each child was informed that he would be playing an interactive game with two addressees in turns; either a toddler ('2-year-old') or a same-age peer (' 5 -year-old'). They were told that the game partners were sitting in other rooms and that they could see the bird token and the digital game board on their monitors. In fact, the confederate performed the role of both addressees, while remaining blind to which one of the two roles he was performing in any given trial. At the onset of each trial the confederate did not know the location of the acorn, and he could infer that location only from the movements of the participant. There were a total of 50 trials ( $\sim 35$ min, Figure 1C). The participant alternately interacted with the two pseudo addressees in blocks of five trials. There were two pairs of fictitious child-toddler addressees, two presentation orders of child-toddler addressees, and two sets of target configurations, counterbalanced over participants. The game was programmed using Presentation (Neurobehavioral Systems, Albany, CA, USA) software on a Windows XP personal computer.

\section{Manipulation of task difficulty}

Task difficulty was manipulated by introducing a disparity between the movements allowed to the bird token (i.e. translations to the center of each square) and the potential location of target object (i.e. the white circles).

Namely, the bird could not be overlaid on the precise location of the acorn when a square contained more than one white circle. 'Easy, medium, and hard trials' 
are distinguished according to their local characteristics, i.e. trials where the acorn was located in a square with one, two, or three white circles, respectively. Importantly, even in relatively easy trials the child needed to find a way to make clear to the Addressee which square contained the acorn, disambiguating it from other squares on the game board, in particular from those squares visited by the bird while moving across the game board. Easy, medium, and hard trials were pseudo-randomly intermixed such that there was an overall increase in difficulty during the course of the experiment. The rationale for manipulating task difficulty was to challenge the participants toward the creation of new communicative behaviors.

The percentage of successfully communicated easy trials was $94.6 \pm 6.7 \%$. Medium and hard trials were successfully communicated on $57.1 \pm 11.7 \%$ and $16.8 \pm 19.9 \%$ of the trials, respectively.

Note that the above mentioned classification scheme is not suitable for objectively and consistently categorizing the communicative difficulty experienced by the participants, since trials with similar local characteristics might actually differ in difficulty. For instance, the top right square of the game board contains the same number of potential target locations (i.e. 2 circles, see Figure 1A) as the square in the middle of the bottom row. However, discriminating between the two circles in the latter square may involve different communicative strategies than discriminating between the two circles in the top right square: The left circle in the bottom middle square may require a detour over other squares to disambiguate it from the top circle in the same square. Besides the number of potential target locations, the exact spatial configuration of those target locations may lead to different communicative strategies and difficulties.

\section{Supplemental Analyses}

In the following sections, we report a number of additional, post-hoc analyses designed to verify the specificity of the findings reported in the main text.

\section{Communicative specificity of the adjustments in children} behavior

We performed additional analyses to verify whether the adjustments reported in the main analysis (Figure 2) were specific to communicative aspects of the children's behavior. The main analysis already addresses this issue by contrasting the time spent on a communicatively relevant location of the game board (i.e. Target location) with the time spent on other locations of the game board (Non-target locations). Here we report the results of additional posthoc comparisons on dependent variables selected for being closer to motoric than communicative aspects of the children's performance. The aim of these comparisons was to assess whether the interaction effect found in the Addressee * Location ANOVA could in fact be a generic motoric effect evoked by the belief of communicating with a Child or a Toddler. We considered the dependent variables of planning times (duration of event 1, Figure 1b), movement times (duration of event 2), the number of moves made by the participants, movemen times of the confederate (duration of event 3), and communicative success. These additional post-hoc tests were calculated for each of the two Addressee types (Toddler, Child) and statistically compared by means of two-sided paired t-tests. It should be emphasized that these post-hoc tests dealt exclusively with one of the main effects considered in the two-way ANOVA (namely, Child vs. Toddler difference), and therefore those tests could not increase the risk of finding a false positive interaction in the two-way ANOVA.

We found that the dependent variables of overall planning and movement times $(p=.13$ and $p=.17)$ and the number of moves made by the participants ( $p=$ .87), as well as performance and response times of the confederate ( $p=.12$ and $p=.10$ ), were matched between both Addressee types. These results support the notion that the adjustments reported in the main analysis (Figure 2) were specific to communicative aspects of the children's behavior.

\section{Temporal dynamics of the communicative adjustments}

We performed an additional analysis to test whether the children's communicative adjustments reported in the main analysis (Figure 2) were present from their first encounter with this communicative setting. The two-way ANOVA with factors Addressee and Location reported in the main text (Figure 2) was 
therefore further qualified by separately testing this interaction in the first and in the second half of the experiment, through a three-way ANOVA with factors Addressee, Location, and Task epoch. There was a significant three-way interaction, $F(1,23)=5.2, p=.033$, indicating that the interaction effect of Addressee and Location on the Time spent on game board locations was present in the first half of the experiment, $F(1,23)=5.9, p=.024$, but not in the second half, $F(1,23)=0.4, p=.56$; see Figure S1. These results indicate that the communicative adjustments reported in the main analysis (Figure 2) were present from the children's first encounter with this communicative setting. In fact, the communicative adjustments were attenuated with experience of the ongoing communicative interaction, in line with the findings of (NewmanNorlund, et al., 2009).

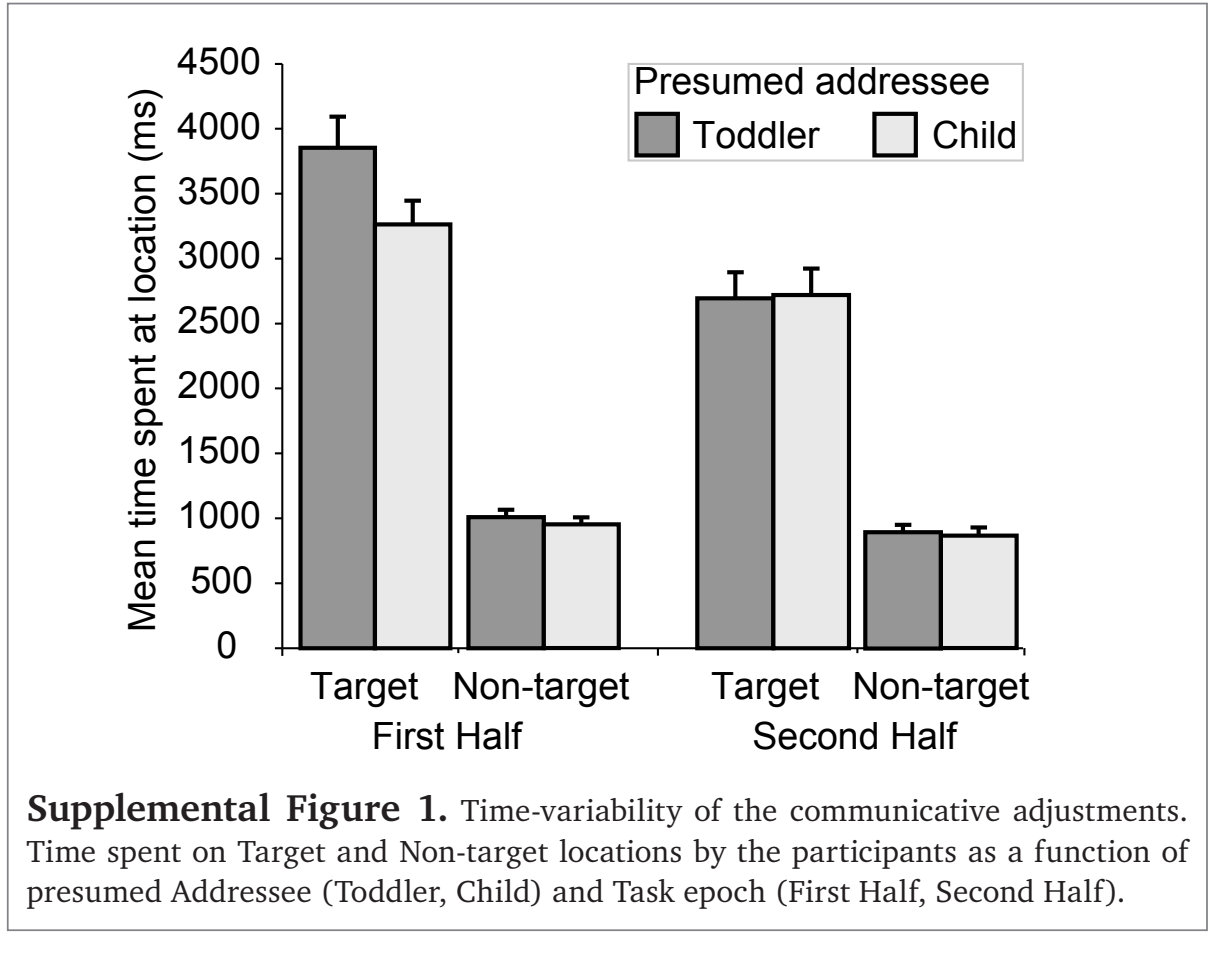

Specificity of the social environmental parameters considered in this study

We performed additional post-hoc analyses to verify the stability and specificity of the predictive relation between daycare attendance and communicative adjustments reported in the main analysis (Figure 3). We assessed the predictive value of a number of additional independent variables (Table S1) on the magnitude of the children's communicative adjustments. These variables capture general measures of task performance, children's experience with audio-visual devices, and derivative measures of the main sources of social interactions experienced by the five-year-olds (i.e. familial and non-familial). We used single linear regression analyses to maximize the sensitivity of these control analyses, at the costs of reduced specificity, in order to assess whether the result obtained in the multiple regression analysis (Figure 3) could in fact be derived from other parameters describing the children's environment or their task performance. Even under these statistically lenient conditions, these alternative parameters were not accounting for significant portions of the children's communicative adjustments. These results support the notion that the predictive value of daycare attendance on communicative adjustments reported in the main analysis (Figure 3) was specific across a number of conceivable parameters.

\begin{tabular}{|c|c|c|c|c|}
\hline & Beta & t-statistic & significance $(p)$ & $\mathbf{R}_{\mathrm{adj}}^{2}$ \\
\hline \multicolumn{5}{|l|}{ Siblings } \\
\hline Number of siblings & -.140 & -0.662 & .515 & -.029 \\
\hline Number of younger siblings & -.125 & -0.125 & .589 & -.029 \\
\hline Number of older siblings & -.011 & -0.053 & .958 & -.045 \\
\hline Cumulated sibling age & .126 & 0.594 & .559 & -.029 \\
\hline Birth order (e.g. first born) & -.011 & -0.053 & .958 & -.045 \\
\hline \multicolumn{5}{|l|}{ Socio-economic status } \\
\hline Mother's education level & .052 & 0.243 & .811 & -.043 \\
\hline Father's education level & -.077 & -0.362 & .721 & -.039 \\
\hline \multicolumn{5}{|l|}{ Task performance } \\
\hline Communicative success & -.163 & -0.774 & .447 & -.018 \\
\hline Relative communicative success & .032 & 0.152 & .881 & -.044 \\
\hline Communicative success easy trials & -.049 & -0.228 & .822 & -.043 \\
\hline Communicative success medium trials & .347 & 1.738 & .096 & .081 \\
\hline Communicative success hard trials & -.282 & -1.379 & .182 & .038 \\
\hline \multicolumn{5}{|l|}{ Other } \\
\hline Time spent at home with computer and television & .231 & 1.112 & .278 & .010 \\
\hline
\end{tabular}



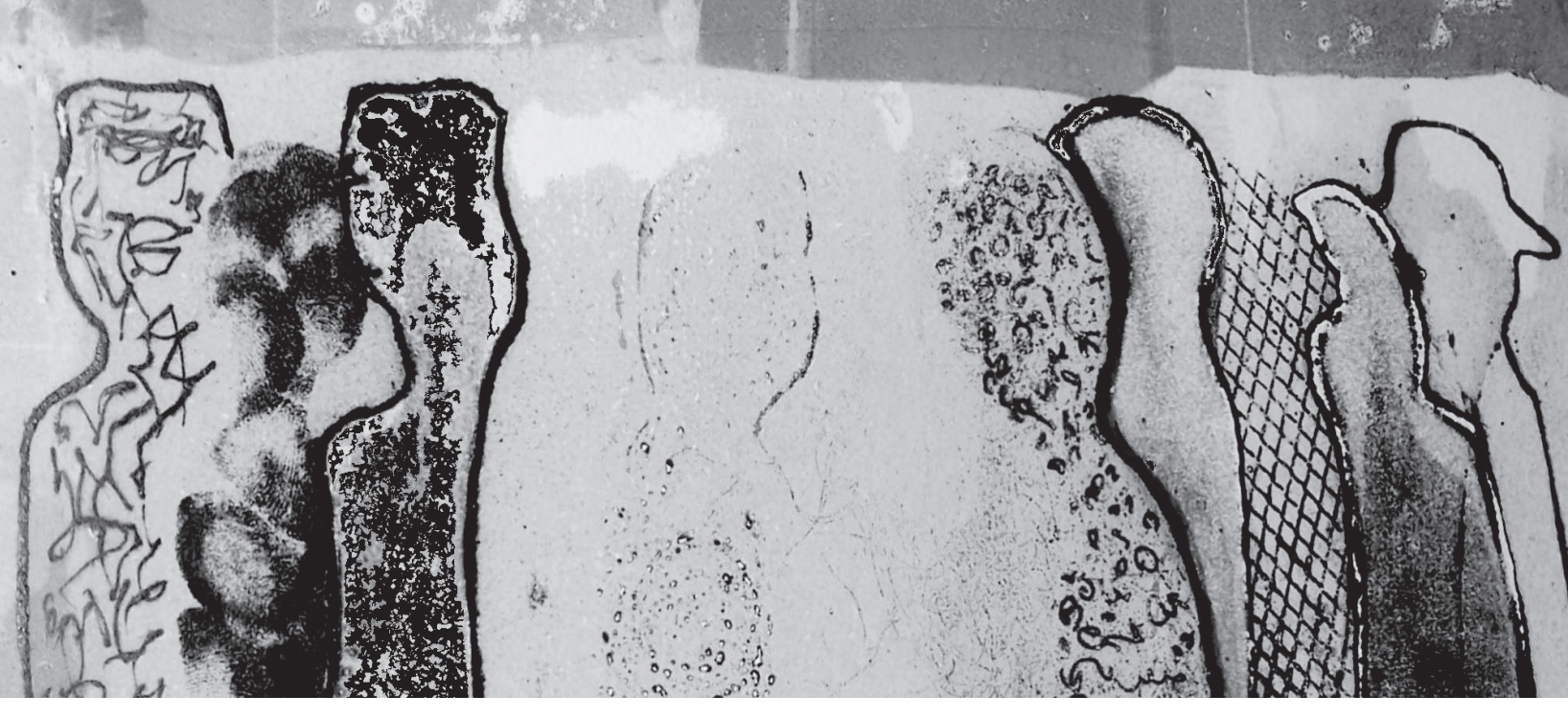

General discussion 
Although a great deal of effort has been spent in studying features and rules of natural language (Chomsky, 1995; de Saussure, 1910-1911; Jackendoff, 2002), undoubtedly improving our understanding of the cognitive structures intrinsic to this faculty (Hauser, et al., 2002), considerably less emphasis has been given to understanding one of the biological functions supported by language, i.e. referential communication (Levinson, 2006; Schilbach, et al., 2013). As outlined in the general introduction, it has been suggested that encodingdecoding schemes, sensorimotor associations, language abilities, reinforcement learning algorithms, automatic interactive alignments, or specialized (social) brain modules support our communicative abilities (Adolphs, 2009; Chomsky, 1995; Hari, Himberg, Nummenmaa, Hamalainen, \& Parkkonen, 2013; Hasson, et al., 2012; Keysers \& Perrett, 2004; Pickering \& Garrod, 2004; Rizzolatti \& Craighero, 2004; Shannon, 1948). However, given the vast number of possible meanings that can be attributed to a communicative action, it remains unclear how symbols can be rapidly selected and interpreted by virtue of any of those mechanisms.

In this thesis, I have addressed the fundamental issue of how meanings are continuously negotiated during communicative interactions. These semiotic negotiations happen continuously: obviously when communicating with others in the absence of a common idiom, but also when learning a language as infants, as well as during most daily conversations. Common words do not contain fixed meanings - they may provide us with clues on a communicative meaning - but are coordinated through an interactive process by which people in dialogue seek and provide evidence that they understand one another (Brennan, et al., 2010). I have addressed this continuous negotiation of meaning by considering human communication as a joint construct of interacting agents, contingent on the interaction dynamics. I have operationalized these fundamental properties of human referential communication through experimental procedures in which people interacted independently from the speech and gestures that are 
often used as behavioral vehicles for communicating mental representations. I have exploited a live interactive task (see BOX 1 in chapter 1 ) in which pairs of participants, as in natural dialogue, continuously need to disambiguate each other's behaviors by taking into account inferred knowledge and beliefs of their interlocutor, conceptual knowledge that presumably accumulates or is sharpened in our minds as we interact. In this final chapter, I will first summarize studies described in the previous chapters and then provide an integrated view of the mechanisms supporting our communicative abilities in the light of the novel findings described in this thesis.

\section{Summary of the empirical findings}

Studying social neural dynamics comes with hard methodological challenges. First, it is necessary to appropriately capture the social dynamics. That means having an experimental platform that combines experimental control with sensitivity to the multidimensional and time-varying features of interpersonal interactions. Second, it is necessary to appropriately capture the neural dynamics. Magnetoencephalography offers the possibility to do that, non-invasively, in humans. However, MEG is also exquisitely sensitive to variations in the position of the head of participants engaged in communicative interactions over multiple experimental sessions. Chapter 2.1 described a set of tools that I have created to overcome those methodological issues, a necessary step for being able to capture functionally specific neuronal markers of human communicative interactions. However, given that head movements pose a fundamental challenge for MEG measurements, these methods might be applied to study cognitive systems other than the main focus of this thesis. Accordingly, I have quantified their use in the context of three different experimental settings, involving somatosensory, visual, and auditory stimuli, assessing both individual and group-level statistics. The online head localization procedure allowed for optimal repositioning of subjects over multiple sessions, resulting in a $28 \%$ reduction of the variance in dipole position and an improvement of up to $15 \%$ in statistical sensitivity. Offline incorporation of the head position time-series into the general linear model resulted in improvements of group-level statistical sensitivity between $15 \%$ and $29 \%$. These tools thus can substantially reduce the influence of head movement within and between sessions, contributing to the sensitivity of many cognitive neuroscience experiments.

Chapter 3 captured the social neural dynamics of the mechanisms supporting our referential communicative abilities, as operationalized with the communication game. In chapter 3.1, I have used MEG to assess spectral, temporal, and spatial characteristics of neural activity evoked when people generate and understand novel shared symbols during live communicative interactions. By directly contrasting those phenomena with a control interaction involving no communicative necessities, I have tested the prediction that both production and comprehension of novel communicative actions rely on shared neural patterns associated with flexible conceptual knowledge. During communicative interactions, two brain regions exhibited significantly stronger power, most pronounced around $55-85 \mathrm{~Hz}$, resulting from broad-band up-regulated neural activity in ventromedial prefrontal cortex and the right temporal cortex. This effect was strongly right-lateralized, emerged already before the occurrence of a particular communicative problem, and well before the observation of communicative actions. These findings sharpen our understanding of the mechanisms supporting human communicative behavior by showing how portions of temporal and ventral prefrontal cortex support both production and comprehension of novel communicative actions with neural activity that predate in time the occurrence of observable communicative events, offering a novel perspective on the implementation of the computations occurring during communicative behavior. In chapter 3.2 I have qualified the characteristics of this flexible conceptual knowledge, showing that it relates to inferred knowledge and beliefs shared across interlocutors. I simultaneously recorded fMRI in two interacting participants engaged in building a pairspecific conversational context, and tested for the cerebral consequences of manipulating common ground dynamics. As common ground emerged within a pair of interlocutors, activity in the right superior temporal gyrus also increased, during both production and comprehension of a communicative action. Using spectral coherence analysis of the BOLD signal across pairs, I have shown that this increased activity was coherent across participants of a pair over periods spanning multiple communicative interactions, well above the occurrence of individual sensory tokens. This slow-frequency within-pair coherency was considerably stronger than that measured in pairs of communicators not sharing a conversational context. These findings indicate that establishing common ground relies on shared, pair-specific reasoning about conceptual features of an ongoing interaction, rather than about the sensory tokens of the interaction.

The studies in chapter 3 , as well as many others dealing with understanding of another agent's intentions, have highlighted several key cortical regions, such as the right posterior superior temporal sulcus (pSTS) and the ventromedial prefrontal cortex (vmPFC). Chapter 4 qualified the contributions of those regions to human communicative abilities by interfering with their neural activity. In chapter 4.1 I have disturbed neural activity in right pSTS to test whether this region is necessary for understanding the meaning of novel communicative actions. Specificity of TMS intervention was ensured by controlling for the communicative relevance of the stimuli and for the cerebral location of the 
intervention. Performance was indexed by Task Efficiency, defined as the number of correct responses per unit of planning time, and by Efficiency Rate, defined as the rate of change (across trials) in Task Efficiency. After rTMS over right pSTS, but not left MT+, the Efficiency Rate of participants was reduced in the communicative game, but not in a visual tracking task. In contrast, after rTMS over left MT+, participants were not able to benefit from experience gained during the previous trials of the visual tracking task (Task X Site interaction). There were no corresponding interactions between tasks and site of rTMS intervention on the Task Efficiency parameter. These findings qualify how right pSTS contributes to understanding the meaning of non-verbal communicative actions. Contradicting previous models of right pSTS contribution to human communication, repetitive TMS over right pSTS did not disrupt the general ability to interpret novel communicative actions. Instead, interference of this region affected the ability to improve task performance. Accordingly, right pSTS appears to be necessary for incorporating previous knowledge, accumulated during interactions with a communicative partner, to constrain the inferential process that leads to action understanding. In chapter 4.2, I have exploited an unfortunate experiment of nature to test whether a lesion in the vmPFC impairs patients' ability to adapt their referential communicative behavior to the presumed age, or cognitive level, of their interlocutor. Specificity of the effects of brain damage was ensured by lesion-symptom mapping and by comparing communicative behaviors evoked by vmPFC patients with behaviors evoked by lesion and healthy control subjects. Ventromedial prefrontal lesion patients communicated as effectively as lesion and healthy controls, but failed to adjust their communicative behavior to both the addressee's performance and presumed abilities. These findings indicate that following a lesion to the ventromedial prefrontal cortex, humans are still motivated and able to select and alter effective communicative actions, but those actions are not guided by a mental model of their current communicative partner. This observation confirms the notion that the ventromedial prefrontal cortex is involved in selecting behaviors guided by a mental model of the social world, and it suggests a cerebral dissociation between selecting an effective communicative action and adjusting the manner of that action to the presumed characteristics of the addressee.

In chapter 5 I have considered a different element of the causal mechanisms supporting our communicative abilities. I took a developmental perspective to understand how the neural mechanisms isolated in chapters 3 and 4 are acquired and shaped. Building on the suggestion that collaborative experiences early in life might be crucial for the emergence of mental coordination abilities, in chapter 5.1 I have assessed the relative contribution of social exposure to familial and non-familial agents on 5-year-olds' referential communicative adjustments to their mental model of an addressee within the communication game. In contrast to linguistic communication, the communicative behaviors evoked under these experimental conditions could not be directly based on previous concrete experiences. Accordingly, the novel situation experienced by the children allowed me to directly tap into their ability to influence the mental states of others through internally generated behaviors. I found that the 5-year-olds spontaneously organized their non-verbal communicative behaviors according to their beliefs about an interlocutor, producing more emphatic communicative actions when they thought they were addressing a two-yearold toddler than when they thought they were addressing an age-peer. Other aspects of their actions were not influenced by their beliefs. The main finding of this study was that the magnitude of these communicative adjustments was predicted by the time spent at daycare, from birth until four years of age, but not by effects of familial social environment. These results suggest that the degree of non-familial social interaction early in life modulates the influence that children's beliefs have on their referential communicative behavior.

\section{Outlook}

This work raises a number of outstanding issues that deserve further investigations. The ability to quickly converge on a common ground of knowledge and beliefs across communicators, efficiently building new and reconfiguring existing semiotic conventions, emerges at different levels of human communication; from infants learning a language without access to the local communicative conventions, to adults with purportedly limited communicative means (shape movements on a game board) as in the studies outlined above. The present work indicates that the meaning of novel shared symbols might be rapidly inferred by embedding those symbols in a conceptual space whose activation predates in time the processing of the symbols themselves (van Berkum, et al., 2008). Even during a simple conversation, we continuously update and sharpen our (conceptual) predictions on sensory material according to the recent history of the communicative interaction. However, it remains a mystery how the human mind constrains the inferential process that leads to action selection and understanding within communicative interaction; i.e. how do we abstract and sort the available information on the basis of relevance? I expect that future investigations on the neural implementation of how representations are constructed from and integrated with incoming stimulus material may provide insight in why individual differences and particular communication failures occur. 
This work also hints at a number of neurocognitive dissociations that will deserve further investigation. For instance, there is an ongoing debate as to whether our theory-of-mind abilities can be subdivided in a cognitive component, supporting our abilities to take into account knowledge and beliefs of another agent, and an affective component, supporting our abilities to take into account the feelings of another agent (Gupta, et al., 2012; Shamay-Tsoory, et al., 2009). Recent investigations from our lab seem to initially support such a dissociation, as when measures of fluid intelligence and systemizing abilities, but not empathy and reward-related tendencies, have been shown to account for significant portions of inter-subject variability in the ability to quickly grasp novel communicative meanings according to recent communicative interactions (e.g. chapter 4.1 and (Volman, et al., 2012)). In contrast, empathy scores appear to be more closely related to audience design abilities (Newman-Norlund, et al., 2009). Taken together, I suggest that while pro-social attitudes (approximately indexed by empathy) might provide the motivational drive necessary for adjusting communicative behavior to a given agent (Tomasello, 2008), other general-purpose cognitive abilities (approximately indexed by fluid intelligence) might provide the computational tools necessary to cope with the complexity of human referential communication (van Rooij, et al., 2011). Studying human development might provide a relevant handle for understanding how those motivational drives and cognitive abilities are implemented and coordinated. In a first attempt to address these issues (chapter 5.1), I have investigated children's ability to influence the mental state of others, and whether these abilities are influenced by the extent and nature of children's social interactions (Carpendale \& Lewis, 2004; de Rosnay \& Hughes, 2006; Dunn \& Shatz, 1989; Hrdy, 2009; Lewis, et al., 1996; Perner, et al., 1994). The rationale and focus of this study is quite different from a large body of existing developmental work that has focused on our ability to attribute mental states to others (BaronCohen, et al., 1985; Wellman, et al., 2001). In a nutshell, my work suggested that referential communicative abilities might be bootstrapped within social interaction itself: 5-year-olds' internally-generated communicative adjustments to their mental model of an addressee were shaped by their early social experience with other cognitive agents. Those results illustrate the relevance of charting the developmental trajectories generated by different sources of social interaction through longitudinal studies with objective measures of the time spent on those interactions. It is known that, in adults, social network size has a positive impact on neural circuits deemed relevant for social cognition, e.g. vmPFC, pSTS, anterior cingulate cortex, and amygdala (Bickart, Wright, Dautoff, Dickerson, \& Barrett, 2011; Kanai, Bahrami, Roylance, \& Rees, 2012; Lewis, et al., 2011; Sallet, et al., 2011). Accordingly, it appears relevant to explore how brain development is influenced by early social experiences that have an impact on our communicative abilities, and whether such effects are long-lasting.

Studying the effects of brain lesion on non-verbal communicative abilities is another dimension that, besides its clinical relevance, might prove extremely fruitful for understanding the neural mechanisms supporting those abilities. For instance, studies of patients suffering from frontotemporal dementia [FTD - a deterioration of the ventral base of the frontal lobe progressing towards the anterior temporallobes (Snowden, Neary, \& Mann, 2002)] reveal that intrinsically motivated social relationships are severely affected when the frontal lobe and right temporal pole degenerate (Fiske, 2010). The behavioral variant of FTD (bvFTD), also referred to as frontal variant FTD (fvFTD), is known for leading patients to fail to recognize that anything is wrong with their behaviors (lack of insight) (Avineri, 2010). The patient is able to reason about social rules even though the same patient has difficulty putting these rules into action (Mikesell, 2010). In a similar vein, patients can understand that others can have different beliefs but perform badly when asked about the emotional state of another (Mates, 2010). Social deficits may be seen during conversational exchanges where a patient is unable to keep track of events occurring during the interaction, and thus is unable to hold a coherent conversation. Semantic dementia, also referred to as temporal variant FTD (tvFTD), is associated with predominantly temporal lobe atrophy, typically greater in the left than in the right hemisphere (Weder, Aziz, Wilkins, \& Tampi, 2007). Studies involving semantic dementia patients show that the anterior temporal lobes are important for accessing knowledge of coherent concepts (Lambon Ralph, et al., 2010). When these patients are shown a picture of a cat and are asked to point out other related items from a list, they point to photos of animals sharing superficial features with the cat, rather than conceptual similarities. For instance, the patients might include furry and long-tailed animals, and exclude tigers and lions. Taken together, these observations point to a degree of specialization between temporal and frontal contributions to human communication: The anterior temporal lobes might be particularly relevant for processing coherent concepts, whereas the frontal cortex might be involved in putting this knowledge into action as when dealing with model-based representations of other agents. This consideration fits with the observation that patients with vmPFC lesions were able to communicate as effectively as control groups, but they did not adjust their communicative behavior to the characteristics and performance of a presumed addressee (chapter 4.2). It remains to be seen whether these considerations on a division of labour between frontal (vmPFC) and anterior temporal cortex generalize to non-communicative decisions (e.g. foraging choices, (Kolling, Behrens, Mars, \& Rushworth, 2012)) and to linguistic communication (Gupta, et al., 2012). 
Finally, it should be emphasized that this thesis has largely focused on empirical observations obtained in the context a highly controlled experimental setup, designed to capture one crucial element of communicative interaction, namely sharing meanings of novel symbols extended over several seconds. It remains to be seen whether this approach is adequate for understanding the theoretical components and the cerebral mechanisms supporting human communication. Certainly, it will be important to test how the present findings generalize to other communicative materials (e.g. linguistic and/or gestural), and to interactive situations where communicative roles can be frequently exchanged, as during natural dialogue.

\section{Conclusion}

This thesis elaborates upon theories of human referential communication and extends these theories to the domain of context-dependent social interaction, an important feature for the generation of novel shared symbols. Functional imaging data, supported by the observation of consequences of brain injury and transient interference with brain function, highlight a fundamental role for right temporal and ventromedial prefrontal brain regions in the coordination and mediation of social cognitive constructs as required for referential communication in social interaction. I have discussed empirical evidence obtained in an interactive communicative setting, showing that generating and comprehending novel shared symbols upregulates the same neuronal mechanisms in these cortical regions known to be crucial for processing conceptual knowledge, across pairs of communicators, and over temporal scales independent from transient sensorimotor events (chapter 3.1). In fact, the neural dynamics of right superior temporal gyrus indicates that those conceptual operations span multiple communicative exchanges, temporally synchronized within a communicating pair, and modulated when novel knowledge is generated among interlocutors (chapter 3.2). During comprehension of novel symbols, the right posterior superior temporal sulcus supports our ability to benefit from recent communicative experiences with a communicative partner (chapter 4.1), whilst during generation, the ventromedial prefrontal cortex is crucial for making communicative adjustments to the inferred knowledge and beliefs of the interlocutor (chapter 4.2). Taken together, these findings indicate that the meaning of a novel symbol arises from a conceptual space dynamically defined by the ongoing interaction, rather than by individual communicative tokens themselves. The figure on the other page summarizes these considerations and the main issues addressed in this thesis.

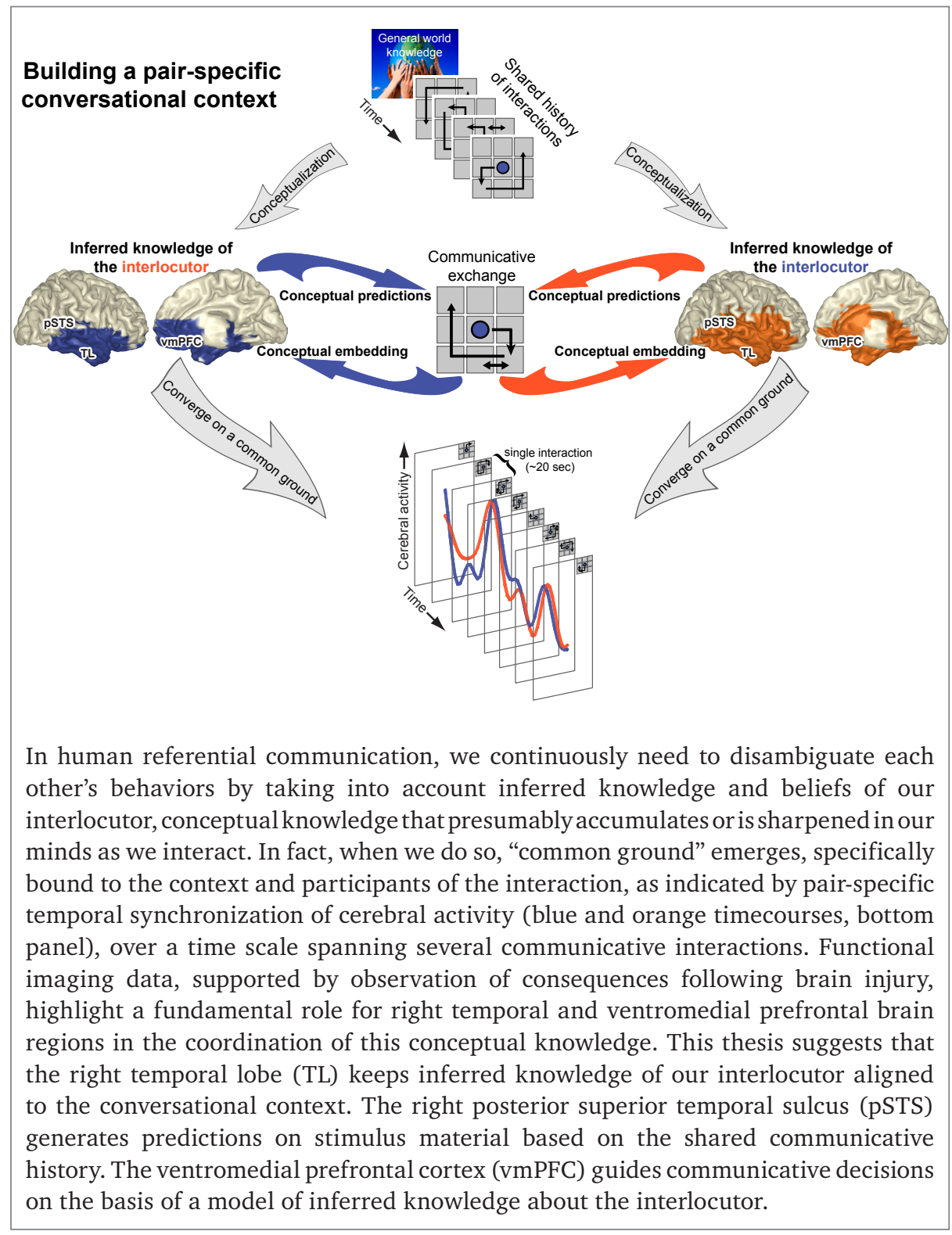



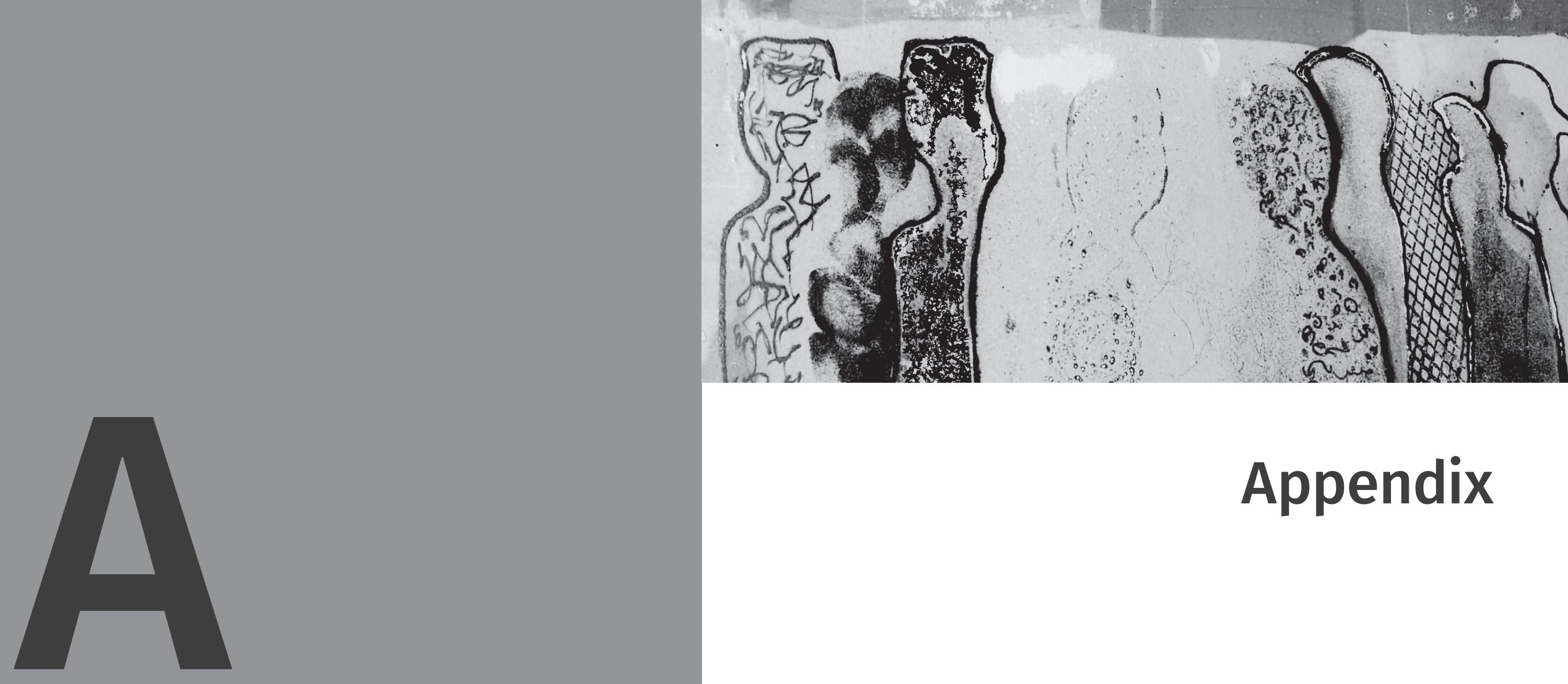

Appendix 


\section{Nederlandse samenvatting}

Stel je voor dat je in een kroeg je lege glas van de tafel pakt en in de lucht houdt. Om deze actie te begrijpen, is het belangrijk de mechanismen te bestuderen die de grijp- en hefbeweging mogelijk maken; bijvoorbeeld hoe patronen van retinale stimulatie vertaald kunnen worden naar veranderingen in momentum van de armspieren. Deze informatie is fundamenteel om de instrumentele aspecten van de beweging te begrijpen, maar een barman die jouw actie observeert zou volledig de plank misslaan wanneer hij alleen deze aspecten van jouw actie in acht zou nemen. Sommige acties, misschien wel de meeste menselijke acties, worden niet alleen geselecteerd en begrepen aan de hand van hun mechanische consequenties, maar vooral ook aan de hand van hun sociale implicaties. Het is heden de vraag hoe de sociale constructen die noodzakelijk zijn om een dergelijke communicatieve actie te begrijpen, in het menselijk brein worden geïntegreerd met de instrumentele aspecten van de actie. Tegelijkertijd is weinig bekend over hoe onze uitzonderlijke communicatieve vaardigheden, wanneer vergeleken met andere diersoorten, cognitief geïmplementeerd en gevormd worden. Dit proefschrift betrekt zich tot het begrijpen van hoe we een actie kunnen selecteren die de 'mentale toestand' van een ander kan beïnvloeden, zoals geschetst door de situatie waarin een klant een drankje kan bestellen door zijn lege glas te heffen in het zicht van een barman.

In deze wetenschappelijke proeve heb ik een van de grote vraagstukken in de sociale wetenschappen geadresseerd. Namelijk, hoe we in staat zijn betekenissen van een communicatieve actie te delen gedurende onze sociale interacties. Onderhandelingen over de betekenis van een communicatieve actie zijn alomtegenwoordig. Een voor de hand liggend voorbeeld is wanneer we met anderen communiceren in de afwezigheid van een gezamenlijke taal (denk aan een buitenlandse toerist), of wanneer we een taal leren als kind. Maar dergelijke onderhandelingen vinden ook plaats tijdens onze dagelijkse 
gesprekken. Gemeenschappelijke woorden bevatten geen vaste betekenissen - ze verschaffen ons hints met betrekking tot een betekenis - maar worden onderhandeld middels een interactief proces waarin de deelnemers aan een dialoog zowel bewijs zoeken als verstrekken omtrent wederzijds begrip. In dit proefschrift heb ik deze continue onderhandeling inzake een communicatieve betekenis geadresseerd door menselijke communicatie als een gezamenlijk construct van de communicatieve partners te beschouwen die gebeurlijk is met de interactiedynamiek. De studie van deze fundamentele eigenschappen van menselijke communicatie heb ik geoperationaliseerd middels experimentele procedures waarin mensen met elkaar interacteren, buiten het bereik van de spraak en gebaren die vaak ingezet worden voor het communiceren van betekenissen. Ik heb gebruik gemaakt van een non-verbale, interactieve communicatie taak (zie BOX 1 in hoofdstuk 1) waarin proefpersoonparen, net als in een alledaags gesprek, continu elkaars acties proberen te begrijpen aan de hand van wat ze denken dat de ander weet, kennis die waarschijnlijk toeneemt of wordt aangescherpt naargelang verdere interacties plaatsvinden. Hier volgt een samenvatting van de empirische studies die beschreven zijn in de voorgaande hoofdstukken, gevolgd door een geïntegreerde beschouwing van de mechanismen die onze communicatieve vaardigheden ondersteunen aan de hand van de nieuwe bevindingen beschreven in dit proefschrift.

De studie van de neurale mechanismen die sociale interactie ondersteunen, brengt zware methodologische uitdagingen met zich mee. Allereerst is het belangrijk op passende wijze de interactiedynamiek te vangen. Hiervoor heeft men een experimenteel platform nodig dat experimentele controle combineert met gevoeligheid voor de multidimensionale en tijdsafhankelijke eigenschappen van interpersoonlijke interactie. Ten tweede is het noodzakelijk de neurale dynamiek te vangen. Magnetoencephalography (MEG) biedt de mogelijkheid om dat te doen, non-invasief, in mensen. Echter, MEG is erg gevoelig voor variaties in hoofdpositie van de proefpersonen wanneer deze gedurende meerdere experimentele sessies met elkaar interacteren. Hoofdstuk 2.1 beschreef een set van gereedschappen die ik gecreëerd heb om deze methodologische obstakels te overwinnen. Een noodzakelijke stap om functioneel specifieke neuronale kenmerken van menselijke communicatieve interacties te vangen. Aangezien hoofdbewegingen een fundamenteel probleem vormen voor MEG metingen in het algemeen, kunnen deze gereedschappen ook toepassing vinden in de studie van andere cognitieve systemen dan beschreven in dit proefschrift. Daarom heb ik de consequenties van toepassing van deze methoden vastgesteld in de context van drie verschillende experimentele situaties, bestaande uit stimulatie van het somatosensorische, het visuele, en het auditieve systeem. Een van de gereedschappen, een hoofdlocalisatie procedure, maakte het mogelijk proefpersonen optimaal te herpositioneren tussen verscheidene meetsessies, resulterende in een $28 \%$ afname van variantie in de localisatie van de neuronale bron en een verbetering van $15 \%$ in de statistische gevoeligheid. Het meenemen van de hoofdpositie informatie in de analyses, door middel van een 'general linear model', resulteerde in een 15 tot $29 \%$ toename van de statistische gevoeligheid op groepsniveau. Deze gereedschappen kunnen dus substantieel bijdragen aan het minimaliseren van de gevolgen van hoofdbewegingen tussen en binnen meetsessies, daarbij bijdragend aan de gevoeligheid van vele cognitieve neurowetenschappelijke experimenten.

Hoofdstuk 3 beschreef de neurale mechanismen die sociale interactie ondersteunen, als gevangen met de communicatie taak. In hoofdstuk 3.1 heb ik MEG gebruikt om zowel spectrale, temporele, als spatiële karakteristieken van neurale activiteit te beschrijven die onderliggend zijn aan het ontstaan van nieuwe gedeelde betekenissen van communicatieve acties, zoals uitgelokt door de communicatie taak. Door deze fenomenen direct te contrasteren met een interactieve controle taak met daarin geen communicatieve noodzaak, testte ik de predictie dat zowel de productie als comprehensie van nieuwe communicatieve acties hetzelfde neurale patroon laten zien. Want, zo luidde de hypothese, in beide gevallen wordt informatie omtrent de kennis van een communicatieve partner in het proces meegenomen. Tijdens de communicatieve interacties, lieten twee hersengebieden significant meer signaal activiteit zien, goed waarneembaar rondom $55-85 \mathrm{~Hz}$ in het spectrum, resulterend door breedbandige, omhoog gereguleerde neurale activiteit in de ventromediale prefrontale cortex en de rechter temporaalkwab. Dit effect was sterk rechts-gelateraliseerd en was al zichtbaar voordat er een afzonderlijke communicatieve handeling plaatsvond. Deze bevindingen verscherpen ons begrip van de mechanismen onderliggend aan onze communicatieve vaardigheden door de laten zien hoe delen van de prefrontaal en de temporaalkwab, gebieden die noodzakelijk zijn voor de verwerking van conceptuele kennis, zowel de productie als comprehensie van nieuwe communicatieve acties ondersteunen. De constatering van neurale activiteit die de communicatieve gebeurtenissen voorafgaat, biedt een nieuw perspectief betreft de computaties die plaatsvinden tijdens communicatieve interacties. In hoofdstuk 3.2 heb ik de eigenschappen van deze conceptuele kennis verder gekwalificeerd, waarbij ik heb laten zien dat het gerelateerd is aan kennis die verondersteld wordt gedeeld te zijn tussen de communicatieve partners. Hiervoor heb ik de hersenactiviteit van twee proefpersonen tegelijk gemeten met twee functional magnetic resonance imaging (fMRI) scanners, terwijl deze proefpersonen bezig waren met het opbouwen van een paarspecifieke conversationele context aan gedeelde betekenissen tijdens een serie van communicatieve interacties. Terwijl er 'common ground' ontstond in de 
proefpersoonparen, nam activiteit toe in de rechter temporaalkwab, zowel tijdens de productie als comprehensie van een nieuwe communicatieve actie. Gebruikmakende van spectrale coherentie analyse van de BOLD signalen van de verschillende proefpersoonparen heb ik laten zien dat deze toenemende activiteit alleen coherent was tussen de proefpersonen van hetzelfde paar, over tijdsperiodes die langer waren dan de tijdsduur van meerdere communicatieve interacties opgeteld. Deze laag-frequente, paar-specifieke koppeling was significant sterker dan in proefpersoonparen die geen communicatieve geschiedenis deelden. Een aantal invloedrijke theorieën betreft menselijke communicatie (zie hoofdstuk 1) legt sterk de nadruk op de specifieke, instrumentele eigenschappen van een communicatieve actie. Echter, bij elkaar genomen, lijken deze bevindingen er eerder op te wijzen dat de betekenis van een communicatieve actie meer afhankelijk lijkt te zijn van conceptuele eigenschappen, gedefinieerd door de paar-specifieke conversationele context, met daarin verondersteld gedeelde betekenissen.

De studies in hoofdstuk 3, evenals andere studies die zich bezig houden met hoe we de intenties van een ander begrijpen, hebben een aantal relevante corticale gebieden op de kaart gezet, zoals de rechter posterieur superieur temporaal sulcus (pSTS) en de ventromediale prefrontaal cortex (vmPFC). Hoofdstuk 4 heeft de bijdrage van deze gebieden aan menselijke communicatieve vaardigheden gekwalificeerd door te interfereren met neurale activiteit in deze gebieden. In hoofdstuk 4.1 heb ik de neurale activiteit in de rechter pSTS verstoord om te testen of dit gebied noodzakelijk is voor het begrijpen van de betekenis van nieuwe communicatieve acties. Specificiteit van de interventie met transcraniële magnetische stimulatie (TMS) werd gewaarborgd door te controleren voor de communicatieve relevantie van de stimuli middels een nietcommunicatieve taak, en voor de locatie van de interventie middels stimulatie van een ander, aangrenzend hersengebied. Observatie van het gedrag liet zien dat er in beide taken sterke leereffecten aanwezig waren. Echter, na stimulatie van de rechter pSTS, maar niet van het controle hersengebied, nam de communicatieve prestatie minder snel toe dan gedurende de controle taak. De proefpersonen konden dus minder goed hun voordeel doen met de ervaring die ze hadden opgedaan tijdens voorgaande communicatieve interacties. Deze bevindingen kwalificeren de rol van de rechter pSTS in het begrijpen van de betekenis van nieuwe non-verbale communicatieve acties door te laten zien dat de rechter pSTS noodzakelijk lijkt voor het incorporeren van kennis opgedaan tijdens eerdere interacties met een communicatieve partner, mogelijk om de betekenis van een communicatieve actie te achterhalen. In hoofdstuk 4.2 heb ik gebruik gemaakt van een ongelukkig experiment van moeder natuur om te testen of een lesie in de ventromediale prefrontale cortex (vmPFC) van invloed is op het vermogen om een communicatieve actie aan te passen aan de veronderstelde leeftijd, en dus het cognitief niveau, van de communicatieve partner. Specificiteit van de effecten van hersenschade werd gewaarborgd door 'lesion-symptom mapping', en door vergelijkenis van de communicatieve handelingen van vmPFC patiënten met die van lesie en gezonde controle proefpersonen. Ventromediale prefrontale lesie patiënten bleken in staat net zo effectief te communiceren als de controle proefpersonen, maar slaagden er niet in rekening te houden met de prestaties en cognitieve vermogens van hun communicatieve partners. In tegenstelling tot de controle proefpersonen legden zij niet meer nadruk tijdens het uitvoeren van hun communicatieve handelingen wanneer ze met een kind dachten te spelen dan wanneer met een volwassen persoon. Deze bevindingen wijzen erop dat na een lesie in de vmPFC, mensen nog steeds gemotiveerd en in staat zijn om effectieve communicatie acties te produceren maar dat deze acties niet worden begeleid door een 'mentaal model' van hun communicatieve partner. Deze observatie bevestigt de stelling uit de literatuur dat de vmPFC betrokken is bij de selectie van gedrag aan de hand van een mentaal model van de sociale omgeving. En het suggereert dat er een cerebrale dissociatie is tussen het selecteren van een effectieve communicatieve actie en het aanpassen van die actie aan de veronderstelde eigenschappen van de communicatieve partner.

In hoofdstuk 5 heb ik een ander element van de causale mechanismen die onze communicatieve vaardigheden ondersteunen in acht genomen. Ik heb hierbij een ontwikkelingsperspectief aangenomen om te begrijpen hoe de neurale mechanismen geïdentificeerd in hoofdstukken 3 en 4, worden verworven en gevormd. Bouwende op de suggestie in de literatuur dat collaboratieve ervaringen vroeg in het leven cruciaal zouden kunnen zijn voor de ontwikkeling van communicatieve vaardigheden, heb ik in hoofdstuk 5.1 de relatieve bijdrage van sociale blootstelling aan familiaire als aan niet-familiaire anderen aan de communicatieve aanpassingen van 5 jaar oude kinderen bepaald. In tegenstelling tot verbale communicatie kunnen de communicatieve acties van de kinderen in de communicatie taak niet direct gebaseerd worden op eerdere concrete ervaringen. Dankzij de nieuwe situatie die de kinderen ervoeren, kon ik dus direct hun vermogen om de mentale toestand van een ander te beïnvloeden, aftappen. Hierbij vond ik dat 5 -jarigen spontaan hun non-verbale communicatieve acties aanpassen aan veronderstelde cognitieve eigenschappen van hun communicatieve partner. Ze legden meer nadruk tijdens het uitvoeren van hun communicatieve handelingen wanneer ze met een fictieve tweejarige peuter dachten te spelen dan wanneer met een leeftijdsgenootje. De hoofdbevinding van deze studie was dat de grootte van deze communicatieve aanpassingen voorspeld werd door de tijd die de kinderen hadden doorgebracht 
op het kinderdagverblijf, vanaf geboorte totdat ze naar school gingen in hun vierde levensjaar. Hierbij vond ik geen effecten van de familiaire sociale omgeving, suggesterende dat vooral vroege niet-familiaire sociale interactie van invloed is op de mate waarin de veronderstellingen van kinderen worden meegenomen in hun communicatief gedrag.

\section{Conclusie}

Deze wetenschappelijke proeve bouwt voort op bestaande theorieën over menselijke communicatie en breidt deze uit naar het domein van contextafhankelijke sociale interactie, een belangrijk ingrediënt voor het ontstaan van nieuwe gedeelde betekenissen. Gegevens aan de hand van metingen van hersenactiviteit, gesteund door observaties van de consequenties van hersenschade en tijdelijkeinterferentievan hersenfunctie, hebben een belangrijke rol aan het licht gebracht voor de prefrontaal en rechter temporaalkwab in de ondersteuning van onze communicatieve vaardigheden. Ik heb uitgewijd over empirisch bewijs, verkregen in een interactieve communicatieve setting, dat laat zien dat zowel de productie als de comprehensie van nieuwe gedeelde betekenissen dezelfde neuronale mechanismen omhoog reguleert in die gebieden die nodig zijn voor de verwerking van conceptuele kennis. Deze neurale processen vonden tegelijk plaats in proefpersonen van hetzelfde paar, gedurende tijdsperioden die langer waren dan de vergankelijke gebeurtenissen tijdens een enkele communicatieve handeling, en werden gemoduleerd door het ontstaan van nieuwe kennis in een paar. Tijdens de comprehensie van communicatieve acties ondersteunt de rechter pSTS door kennis te leveren aan de hand van de recente communicatieve geschiedenis met een communicatieve partner, terwijl tijdens de productie van communicatieve acties, de vmPFC noodzakelijk is voor het maken van communicatieve aanpassingen naar de veronderstelde kennis en niveau van de communicatieve partner. Bij elkaar genomen, lijken de bevindingen in dit proefschrift erop te wijzen dat de betekenis van een communicatieve boodschap ontstaat aan de hand van conceptuele eigenschappen van die boodschap, dynamisch gedefinieerd tijdens voortdurende interactie met een communicatieve partner, dan door de boodschap zelf. 


\section{References}

Adjamian, P., Barnes, G. R., Hillebrand, A Holliday, I. E., Singh, K. D., Furlong, P. L., Route, P. J. (2004). Co-registration of magnetoencephalography with magnetic

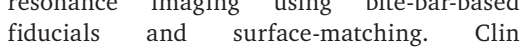
Neurophysiol, 115(3), 691-698.

Adolphs, R. (2009). The social brain: neural basis of social knowledge. Annu Rev Psychol, 60, 693-716.

Amodio, D. M., \& Frith, C. D. (2006). Meeting of minds: the medial frontal cortex and socia

Anstey, K. J, Salim, A. Lord S. R. 20. Mitchell, P., Mill, K. \& Von Sanden, C. (2007). Our correct use of ANCOVA yields acceptable results - Response. Journal of the International Neuropsychological Society, 13(2), 371-371.

Arfeller, C., Schwarzbach, J., Ubaldi, S., Ferrari, P., Barchiesi, G., \& Cattaneo, L. (2013). Wholebrain haemodynamic after-effects of $1-\mathrm{Hz}$ magnetic stimulation of the posterior superior temporal cortex during action observation. Brain Topogr, 26(2), 278-291.

Arieli, A., Sterkin, A., Grinvald, A., \& Aertsen, A. (1996). Dynamics of ongoing activity: enticis 1868-1871

Avineri, N. (2010). The Interactive Organization of 'Insight': Clinical Interviews with Frontotemporal Dementia Patients. In A. W. Mates, L. Mikesell \& M. S. Smith (Eds.), Language, Interaction and Frontotemporal Dementia: Reverse Engineering the Social
Bahnemann, M., Dziobek, I., Prehn, K., Wolf, I., \& Heekeren, H. R. (2010). Sociotopy in the emporoparietal cortex: common in the distinct processes Social Cognitive and Affective Neuroscience, 5(1), 48-58.

Bandettini, P. A., Jesmanowicz, A., Van Kylen, J. Birn, R. M., \& Hyde, J. S. (1998). Functiona MRl of brain activation induced by scanner acoustic noise. Magn Reson Med, 39(3),

gh, J. A., Chen, M., \& Burrows, L. (1996). Automaticity of social behavior: direct effects (

ron-Cohen, S., Leslie, A. M. \& Frith, U. (1985) Does the autistic child have a "theory of mind"? Cognition, 21(1), 37-46.

Barr, D. J. (2004). Establishing conventional communication systems: Is common knowledg

Bates, E., Wilson, S. Me Science, 28(6), 937-962. Sereno, M. ... Knight, R. T., \& Dronkers, N. F. (2003). Voxel-based lesion-symptom mapping. Nat Neurosci, 6(5), 448-450.

Bechara, A., Damasio, A. R., Damasio, H., \& Anderson, S. W. (1994). Insensitivity to future prefrontal cottex. Cogition, 50(1-3), 7-15

Beer, J. S., John, O. P., Scabini, D., \& Knight, R. T. (2006) Orbitofrontal cortex and socia behavior: integrating self-monitoring and emotion-cognition interactions. J Cogn Neurosci, 18(6), 871-879

Behabadi, B. F., Polsky, A., Jadi, M., Schiller, J., \& Mel, B. W. (2012). Location-dependent excitatory synaptic interactions in pyramida neuron dendrites. PLoS Comput Biol, 8(7),
Behrens, T. E., Hunt, L. T., \& Rushworth, M. F. (2009). The computation of socid

Behrens, T. E., Hunt, L. T., Woolrich, M. W., \& Rushworth, M. F. (2008). Associative learning of social value. Nature, 456(7219), 245-249.

Beintema, J. A., \& Lappe, M. (2002). Perception of biological motion without local image motion.

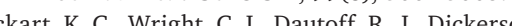

B. C. \& Bart, L. F. (2011), A. J., Dickerson, and social network size in humans. Nat Neurosci, 14(2), 163-164.

Bird, C. M., Castelli, F., Malik, O., Frith, U., \& Husain M. (2004). The impact of extensive medial frontal lobe damage on 'Theory of Mind' and cognition. Brain, 127(Pt 4), 914-928.

Blanke, O., Mohr, C., Michel, C. M., Pascual-Leone, A., Brugger, P., Seeck, M., . . . Thut, G. (2005). Linking out-of-body experience and self processing to mental own-body imagery at the temporoparietal junction. Journal of
Neuroscience, 25(3), 550-557.

P. Toni, ${ }^{2}$ \& design in human communication: Recipient heuristics or perspective taking? Front Hum Neurosci, 6, 253.

Blumhagen, F., Zhu, P., Shum, J., Scharer, Y. P., Yaksi, E., Deisseroth, K., \& Friedrich, R. W. (2011). Neuronal filtering of multiplexed odour representations. Nature, 479(7374), 493-498.

Boroojerdi, B., Prager, A., Muellbacher, W., \& Cohen, L. G. (2000). Reduction of human visual cortex excitability using $1-\mathrm{Hz}$ transcranial magne stim

Jon Contributions of the human temporoparietal
junction and MT/V5+ to the timing interception revealed by transcranial magnetic stimulation. J Neurosci, 28(46), 12071-12084.

Brand, R. J., Baldwin, D. A., \& Ashburn, L. A. (2002). Evidence for 'motionese': modifications in mothers' infant-directed action. Developmental Science, 5(1), 72-83

Brennan, S. E., Galati, A., \& Kuhlen, A. (2010). Two minds, one dialog: Coordinating speaking and understanding. In B. Ross (Ed.), Psychology of Learning and Moivation (Vol. 53, pp. 301-344). Brodsky, P., Wate computational structure of child directed anguage. In D. McNamara \& J. Trafton (Eds), Proceedings of the 29th Cognitive Science Society Conference. Austin, TX: Cognitive Science Society.
Burkart, J. M., Hrdy, S. B., \& Van Schaik, C. P. (2009) Cooperative Breeding and Human Cognitive 175-186

Buzsaki, G., Anastassiou, C. A., \& Koch, C. (2012). The origin of extracellular fields and currents-EEG, ECoG, LFP and spikes. Nat Rev Neurosci, $13(6), 407-420$.

X. J. (2012). Mechanisms of cillations. Annu Rev Neurosci, 35,

Cacioppo, J. T., Petty, R. E., \& Kao, C. F. (1984). The Efficient Assessment of Need for Cognition. Journal

Camerer, C. F. (2003). Behavioural studies of strategic thinking
$7(5), 225-231$. C., Acik, A., Konig, P., Engel, A. K., \& Hipp, J. F. (2012). The saccadic spike artifact in MEG. Neuroimage, 59(2), 1657-1667.

Carpendale, J. 1., \& Lewis, C. (2004). Constructing children's social understending within social interaction. Behavioral and Brain Sciences, 27(1), 79-96; discussion 96-151.

Carpenter, P. A., Just, M. A., \& Shell, P. (1990) What One Intelligence-Test Measures - a Theoretical Account of the Processing in the Review, 97(3), 404-431.

Carruthers, P. (1996). Simulation and selfknowledge: a defence of theory-theory. In P. Carruthers \& P. K. Smith (Eds.), Theorie of theories of mind (pp. 22-38). Cambridge: Cambridge University Press.

MIT Press.

E. Braghitoni, D \& di Pellegrino, G. (2012). It Is the Outcome that Counts Damage to the Ventromedial Prefrontal Cortex Disrupts the Integration of Outcome and Belie Information for Moral Judgment. Journal o the Internationa $962-971$. Peli, E, Maccioli, M., Ladavas, E., \& Persino, G. (2007). Selective deficit in personal moral judgment following damage
to ventromedial prefrontal cortex. Soc Cogn Affect Neurosci, 2(2), 84-92.

Ciaramelli, E, Sperotto, R. G Mattioli, F, \& di Pellegrino, G. (2013). Damage to the interpersonal disgust. Soc Cogn Affect Neurosci, 8(2), 171-180.

Clark, H. H. (1996). Using language. Cambridge, England: Cambridge University Press. 
Cole, K., \& Mitchell, P. (2000). Siblings in the development of executive control and a theory of mind. British Journal of Development Psychology, 18, 279-295.

Cutica, I., Bucciarelli, M., \& Bara, B. G. (2006). Neuropragmatics: Extralinguistic pragmatic ability is better preserved in left-hemispheredamaged patients than in right-hemispheredamaged patients. Bran Lang, 98(1), 12-25. Cutting, $A, L$, \& Din, background: individual differences and interrelations. Child Dev, 70(4) 853-865.

Dale, A. M., \& Sereno, M. I. (1993). Improved Localization of Cortical Activity by Combining Eeg and Meg with Mri Cortical Surface Reconstruction - a Linear-Approach. Journal of Cognitive Neuroscience, 5(2), 162-176.

Danchin, E., Giraldeau, L. A., Valone, T. J., \& Wagner, R. H. (2004). Public information: from nosy neighbors to cultural evolution. Science,

Dawkins, R., \& Krebs, J. (1978). Animals signals \& D. NB (Eds.), Behvion. In Krebs $J$ evolutionary approach (pp. 282-309). Oxford: Blackwell.

de Langavant, L. C., Remy, P., Trinkler, I., McIntyre, J., Dupoux, E., Berthoz, A., \& Bachoud-Levi, A. C. (2011). Behavioral and Neural Correlates of Communication via Pointing. PLoS One, 6(3).

de Lange, F. P., Spronk, M., Willems, R. M., Toni, I., Bekkering, H. (2008). Complementary systems for understanding action intentions. Curr Biol, $18(6), 454-457$

de Munck, J. C., Verbunt, J. P., Van't Ent, D., \& Van Dis 3 digitizer and motion monitoring syster. Phys Med Biol, 46(8), 2041-2052.

de Rosnay, M., \& Hughes, C. (2006). Conversation and theory of mind: Do children talk their way to socio-cognitive understanding? British Journal of Developmental Psychology, 24, 7-37.

de Ruiter, J. P., Noordzij, M. L., Newman-Norlund, S., Newman-Norlund, R., Hagoort, P., Levinson, $\mathrm{S}$. C., \& Toni, I. (2010). Exploring the cognitive infrastructure of comminatcation. Interaction Studies, 11(1), 51-7

de Saussure, F. (1910-1911). [Saussure's Lectures on (a)

Ansing Designs. Multivarite in Repeated Measures 16(1), 105-123.
Derix, J., Iljina, O., Schulze-Bonhage, A., Aertsen, A., \& Ball, T. (2012). "Doctor" or "darling"? Decoding the communication partner from ECoG of the anterior temporal lobe during nonexperimental, real-life social interaction. Fron Hum Neurosci, 6, 25

Drager, B., Breitenstein, C., Helmke, U., Kamping, S. $\&$ Knecht, S. (2004). Specific and nonspecific picture-word verification. European Journal of Neuroscience, 20(6), 1681-1687.

Duecker, F, \& Sack, A. T. (2013). Pre-stimulus shan TMS facilitates target detection. PLoS One, 8(3), e57765.

Dunn, J., \& Shatz, M. (1989). Becoming a Conversationalist Despite (or Because of Having an Olde
$60(2), 399-410$.

Egidi, G., \& Caramazza, A. (2013). Cortical system for local and global integration in discours comprehension. Neuroimage, 71, 59-74.

Evans, N., \& Levinson, S. C. (2009). With diversity in mind: Freeing the language sciences from Sciences, 32(5), 472-492

Feiler, L., \& Camerer, C. F. (2010). Code Creation in Endogenous Merger Experiments. Economic Inquiry, 48(2), 337-352.

Fiske, A.P. (2010). Dispa Fails to Sustain Social Relationships. In A. W. Mates, L. Mikesell \& M. S. Smith (Eds.) Language, Interaction, and Frontotempora Dementia: Reverse Engineering the Socia

Fodor, J. A. (2000). The Mind Doesn't Work That Way. Cambridge, MA: MIT Pres

(2010). The free-energy principle: a 127-138.

Friston, K., Kilner, J., \& Harrison, L. (2006). A free energy principle for the brain. Journal of Physiology-Paris, 100(1-3), 70-87.

Friston, K. J., Holmes, A., Poline, J. B., Price, C. J., \& Frith, C. D. (1996). Detecting activations in PET and FMRr. levels of inference and power. Neuroimage, 4 (3 Pt 1), 223-235.

Frith, C. D., \& Frith, U. (1999). Interacting minds-a biological basis. Science, 286(5445),

rith, C. D., \& Frith, U. (2006). The neural basis of C. D. \& Fing. Neuron, 50(4), 531-534.

neurophysiology of mentalizing Philos Trans Soc Lond B Biol Sci, 358(1431), 459-473.
Gaetz, W., Otsubo, H., \& Pang, E. W. (2008). Magnetoencephalography for clinical pediatrics: the effect of head positioning on Clin Neurophysiol, 119(8), 1923-1933.

Galantucci, B. (2005). An experimental study of the emergence of human communication systems. Cognitive Science, 29(5), 737-767.

Galantucci, B., \& Garrod, S. (2011). Experimental semiotics: a review. Frontiers in Human

ao, T., Scholl, B. J.,

(2012) Dissociating the Detection of Intentionality Temporal Sulcus. Journal of Neuroscience, 32(41), 14276-14280

Garrod, S., \& Pickering, M. J. (2004). Why is conversation so easy? Trends Cogn Sci, 8(1), $8-11$

Gentner, D. (2003). Why we're so smart. In D. Gentner \& S. Goldin-Meadow (Eds.), Language in mind. Advances in the study of language and Press (pp. 195-235). Cambidge, MA: MIT

Giese, M. A., \& Poggio, T. (2003). Neural mechanisms or the recognition of biological movements. Nat Rev Neurosci, 4(3), 179-192.

Goldstone, R. L., \& Rogosky, B. J. (2002). Using relations within conceptual systems to translate across

Grezes, J., Frith, C. D., \& Passingham, R. E. (2004). Inferring false beliefs from the actions of oneself and others: an fMRI study. Neuroimage,

21(2), 744-750.
Grieser, D. L., \& Kuhl, P. K. (1988). Maternal Speech to Infants in a Tonal Language - Support fo Developmental Psychology, 24(1), 14-20.

Gross, J., Kujala, J., Hamalainen, M., Timmermann, L., Schnitzler, A., \& Salmelin, R. (2001). tudying neural interactions in the human brain. Proc Natl Acad Sci U S A, 98(2), 694-699.

Grossman, E. D., Battelli, L., \& Pascual-Leone, A. (2005). Repetitive TMS over posterior STS disrupts perception of biological motion. Vision

Gupta, R., Tranel, D., \& Duff, M. C. (2012). Ventrome does ground in social interaction: implications for seory of mind. Neuropsychologia, 50(1), 145-152.

Haegens, S., Luther, L., \& Jensen, O. (2012). Somatosensory anticipatory alpha activity increases to suppress distracting input. J Cogn Neurosci, 24(3), 677-685
Haegens, S., Osipova, D., Oostenveld, R., \& Jensen, O. (2010). Somatosensory working memory performance in humans depends on both engagement and disengagement of regions a distributed network. Hum Brain Mapp, 31(1)

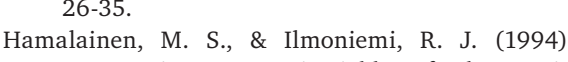
Interpreting Magnetic-Fields of the Brain (1) 35-42.

M., \& Parkerg, T., Nummenmaa, L., Hamalainen M., \& Parkkonen, L. (2013). Synchrony of
brains and bodies during implicit interpersonal interaction. Trends in Cognitive Sciences, 17(3), 105-106.

Hasson, U., Ghazanfar, A. A., Galantucci, B Garrod, S., \& Keysers, C. (2012). Brain-tobrain coupling: a mechanism for creating an sharing a social world. Trends in Cognitive

Hasson, U., Yang, E., Vallines, I., Heeger, D. J., \& receptive windows in human cortex of temporal 28(10), 2539-2550.

Hauser, M. D., Chomsky, N., \& Fitch, W. T. (2002) The faculty of language: what is it, who has) it, and how did it evolve? Science, 298(5598), 1569-1579.

He, B. J. (2011). Scale-free properties of the functional magnetic resonance imaging signal during rest and task. J Neurosci, 31(39), $13786-13795$.

He, B. J., Zempel, J. M., Snyder, A. Z., \& Raichle, M. E. (2010). The temporal structures an functional significance of scale-free brai

. overt language production in functional magnetic resonance imaging. Neuroreport 17(6), 579-582.

Hermes, D., Miller, K. J., Vansteensel, M. J. (201) E. J., Leijten, F. S., \& Ramsey, N. . (2012). Neurophysiologic correlates of fMRI in 1689-1699.

He 1689-1699. Hare B \& Tomasello (a) the cultural intelligence hypothesis. Science, 317(5843), 1360-1366.

Holler, J., \& Stevens, R. (2007). The effect of common ground on how speakers use gesture and speech to represent size information.
Journal of Language and Social Psychology, 26(1), 4-27. 
Holmes, H. A., Black, C., \& Miller, S. A. (1996). A Cross-Task Comparison of False Belief Understanding in a Head Start Po
Exp Child Psychol, 63(2), 263-285.

Hoogenboom, N., Schoffelen, J. M., Oostenveld, R., Parkes, L. M., \& Fries, P. (2006). Localizing human visual gamma-band activity in frequency, time and space. Neuroimage, 29(3),
764-773.

Horton, W. S., \& Keysar, B. (1996). When do speakers take into account common ground? Cognition, take into acco
59(1), 91-117.

Hrdy, S. B. (2009). Mothers and others: the evolutionary origins of mutual understanding. Cambridge, Mas
University Press.

Iacoboni, M. (2005). Neural mechanisms of imitation. Curr Opin Neurobiol, 15(6), 632-637.

lacoboni, M., Molnar-Szakacs, I., Gallese, V., Buccino, G., Mazziotta, J. C., \& Rizzolatti, G. (2005). Grasping the intentions of others with one's own mirror neuron system. PLoS Biol,
3(3), e79.

its in. (2002). Information: Its interpretation, its inheritance, and its sharing. Philosophy of

Brain, R. (2002). Foundations of Language: Brain, Meaning, Grammar, Evolu
Oxford: Oxford University Press

Jacobs, N., \& Garnham, A. (2007). The role of conversational hand gestures in a narrative task. Journal of Memory and Language, 56(2), 291-303.

Jakobs, O., Langner, R., Caspers, S., Roski, C., Cieslik, E. C., Zilles, K., - . Eickhoff, S. B. (2012). Across-study and within-subject functional junction subregion involved in stimulus-context integration. Neuroimage, 60(4), 2389-2398.

Jarsky, T., Roxin, A., Kath, W. L., \& Spruston, N. (2005). Conditional dendritic spike of hippocampal CA1 pyramidal neurons. Nat Neurosci, 8(12), 1667-1676.

Jiang, J., Dai, B., Peng, D., Zhu, C., Liu, L., \& Lu, C. (2012). Neural synchronization during faceto-face communication. J Neurosci, 32(45), 16064-16069.

Kaczmarek, B. L. (1984). Neurolinguistic analysis of verbal utterances in patients with focal les

Kanai, R., Bahrami, B., Roylance, R. \& Rees, (2012). Online social network size is reflected in human brain structure. Proceedings of the Royal Society B-Biological Sciences, 279(1732), 1327-1334.
Keren, A. S., Yuval-Greenberg, S., \& Deouell, L. Y. (2010). Saccadic spike potentials in gammaband EEG: chara suppression. Neuroimage, 49(3), 2248-2263.

Keysar, B., \& Horton, W. S. (1998). Speaking with common ground: from principles to processes in pragmatics: a reply to Polichak and Gerrig. Cognition, 66(2), 191-198.

(20)ers, C., \& Perrett, D. I. (2004). Demstifying Con

irby, S., \& Hurford, J. (2002). The emergence of
linguistic structure: An overview of the iterated learning model. In A. Cangelosi \& D. Paris (Eds.), Simulating the Evolution of Language (pp. 121-148). London: Springer.

Knosche, T. R. (2002). Transformation of wholehead MEG recordings between different sensor positions. Biomed Tech (Berl), 47(3), 59-62.

Kolling, N., Behrens, T. E., Mars, R. B., \& Rushworth, M. F. (2012). Neural mechanisms of foraging. Science, $336(6077), 95-98$.

Kominis, I. K., Kornack, T. W., Allred, J. C., \& multich, M. V. (2003). A subfemtotesla 422(6932), 596-599.

Maguire, E. A. (2009). Jr. J., Hassabis, D., \& of conceptual knowledge during huma decision making. Neuron, 63(6), 889-901.

Lambon Ralph, M. A., Sage, K., Jones, R. W., \& Mayberry, E. J. (2010). Coherent concepts are computed in the anterior temporal lobes. Proc Natl Acad Sci U S A, 107(6), 2717-2722.

Larkm, M. (2012). Acelly

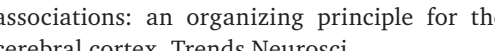
Cr. Y. Honey, C. J. Silbert, L. J. \&

(2011). Topographic mapping of \& Hasson, U. emporal receptive windows using a narry of story. J Neurosci, 31(8), 2906-2915.

Leslie, A. M., Friedman, O., \& German, T. P. (2004) Core mechanisms in "theory of mind". Trends Cogn Sci, 8(12), 528-533.

Levinson, S. C. (2006). On the human interactional engine. In N. Enfield \& S. Levinson (Eds.), Roots of Human Sociality (pp. 39-69): Ber Publisher

Lewis, C., Freeman, N. H., Kyriakidou, C., Maridaki Kassotaki, K., \& Berridge, D. M. (1996). Social sibling influences or general apprenticeship? Child Development, 67(6), 2930-2947.

Lewis, P. A., Rezaie, R., Brown, R., Roberts, N., \& Dunbar, R. I. (2011). Ventromedial prefrontal and social network size. Neuroimage, 57(4) 1624-1629.
Litvak, V., Zeller, D., Oostenveld, R., Maris, E., Cohen, A., Schramm, A., ... Classen, J. (2007). LTP-like changes induced by paired associative stimulation of the primary somatosensory cortex in humans: source analysis and associated changes in b
Neurosci, 25(9), 2862-2874.

Logothetis, N. K., Pauls, J., Augath, M., Trinath, T. \& Oeltermann, A. (2001). Neurophysiologica investigation of the basis of the fMRI signal. Nature, 412(6843), 150-157.

Luczak, A., Bartho, P., \& Harris, K. D. (2013). Gating of sensory input by spontaneous cortical activity. J Neurosci, 33(4), 1684-1695.

Machizawa, M. G., \& Driver, J. (2011). Principal component analysis of behavioural individual differences suggests that particular aspects of visual working memory may relate to specific aspects of attention. Neuropsychologia, 49(6),

Maclaren, J., Herbst, M., Speck, O., \& Zaitsev, M. (2012). Prospective motion correction in brain likovic, A, Amunts, K, Schleich, A.,

Malikovic, A., Amsts, K., Schleicher, A., Mohlberg, (2007). Cytoarchitectonic a... Zilles, K human extrastriate cortex in the region of V5/ MT+: a probabilistic, stereotaxic map of area hOc5. Cereb Cortex, 17(3), 562-574.

Manning, J. R., Jacobs, J., Fried, I., \& Kahana, M. J. (2009). Broadband shifts in local field potential power spectra are correlated with single-neuron spikng in arumans. J Neurosci,

$\begin{array}{lll}\begin{array}{ll}\text { 29(43), } \\ \text { Maratsos, M. P. P. }\end{array} & \text { (1973). Nonegocentric }\end{array}$ Communication Abilities in Preschool-

(2007). No, 697-700.

statistical testing of EEG- and MEG-data. J Neurosci Methods, 164(1), 177-190.

Marshall, P. J., Bouquet, C. A., Thomas, A. L., \& Shipley, T. F. (2010). Motor contagion in young children: Exploring social influences on perception-action coupling. Neural Netw, 23(8-9), 1017-1025.

Mashal, N., Faust, M., Hendler, T., \& Jung-Beeman, M. (2007). An fMRI investigation of the neural correlates underlying the processing of nove metaphoric express $100(2), 115-126$.

Mates, A. W. (2010). Using Social Deficits in Frontotemporal Dementia to Develop a Neurobiology of Person Reference. In A W. Mates, L. Mikesell \& M. S. Smith (Eds.), Dementia: Reverse Engineering the Social Mind.
Maus, G. W., Ward, J., Nijhawan, R., \& Whitney, D. (2012). The Perceived Position of Movin Objects: Transcranial Magnetic Stimulation o Area MT+ Reduces the Flash-Lag Effect. Cere Cortex.

McIntyre, J., Stratta, F., \& Lacquaniti, F. (1998) Short-term memory for reaching to visual targets: psychophysical evidence for body 8423-8435.

Medvedovsky, M., Taulu, S., Bikmullina, R., \& Paetau, R. (2007). Artifact and head movement compensation in MEG. Neurol Neurophysio Neurosci, 4 .

Menenti, L., Pickering, M. J., \& Garrod, S. C. (2012). Toward a neural basis of interactive Neuroscience, 6, 185

Mikesell, L. (2010). Examining Perservative Behaviors of a Frontotemporal Dementia Patient and Caregiver Responses: The Benefits of Observing Ordinary Interactions and L. Mikesell \& M. S. Smith (Eds), W. Mates, . Reverse Engineering the Social Mind

Miller, K. J. (2010). Broadband spectral change: evidence for a macroscale correlate of
population firing rate? J Neurosci, 30(19), 6477-6479

Miller, K. J., denNis, M., Shenoy, P., Miller, W., Rao, R. P., \& Ojemann, J. G. (2007) W. electrocorticography. Neuroimage, $37(2)$

iller, K. J., Hermes, D., Honey, C. J., Sharma, M., Rao, R. P., den Nijs, M., ... . Leuthardt, population activity by rhythm phase in human occipital cortex during a visual search task. Front Hum Neurosci, 4, 197.

Miller, K. J., Leuthardt, E. C., Schalk, G., Rao, R. P. G. G. (2007). Spectral changes in cortical surface 27(9), 2424-2432.

Miller, K. J., Sorensen, L. B., Ojemann, J. G., \& den Nijs, M. (2009). Power-law scaling in the bra surface electric potential. PLoS Comput Bio (12), e

man, J. (2001). Ventromedia prefrontal cortex lesions in humans eliminate Neuroscience, 21(12), RC150.

Mitchell, J. P., Ames, D. L., Jenkins, A. C. \& Banaj, M. R. (2009). Neural Correlates of Stereotype Application. Journal of Cognitive Neuroscience, 21(3), 594-604. 
Mitchell, J. P., Macrae, C. N., \& Banaji, M. R. (2006). Dissociable medial prefrontal contributions to judgments of similar and dissimilar others. Neuron, 50(4), 655-663.

Morosan, P., Rademacher, J., Schleicher, A., Amunts, K., Schormann, T., \& Zilles, K. (2001). Human primary auditory cortex: cytoarchitectonic subdivisions and mapping into a spatial

Mottaghy, F. M., Keller, C. E., Gangitano, M., Ly, J., Thall, M., Parker, J. A., \& Pascual-Leone, and treatment effects of repetitive transcranial magnetic stimulation in depressed patients. Psychiatry Research-Neuroimaging, 115(1-2), 1-14.

Newman-Norlund, S. E., Noordzij, M. L., NewmanNorlund, R. D., Volman, I. A., Ruiter, J. P., Hagoort, P., \& Toni, I. (2009). Recipient design in tacit communication. Cognition, 111(1),

Nichols, S., \& Stich, S. P. (2003). Mindreading: an integrated account of pretence, self-awareness, Clarendon press. Nichols, T., Brett, M., Andersson, J., Wager, T., \&
Poline, J. B. (2005). Valid conjunction inference with the minimum statistic Neuroimage, with 3 , $653-660$. Fernandez, G., \& Jensen, O. (2008). Visual areas become less engaged in associative recall following memory stabilization. Neuroimage, 40(3), 1319-1327.

Nilsen, E. S., \& Graham, S. A. (2009). The relations between children's communicative Cogn Psychol, 58(2), 220-249.

ilsen, E. S., \& Graham, S.

development of preschoolers' (2012). The communicative ambiguity. Child Dev, 83(4) 1400-1415.

Nilsen, E. S., Graham, S. A., Smith, S., \& Chambers, C. G. (2008). Preschoolers' sensitivity to referential ambiguity: evidence for a dissociation between implicit understanding and explicit behavior. Dev Sci, 11(4), 556-562.

Nixon, S. J., Lawton-Craddock, A., Tivis, R., \& Ceballos, N. (2007). Nicotine's effects on Clinical and Experimental Research, 31(12), 2083-2091.

Nolte, G. (2003). The magnetic lead field theorem in the quasi-static approximation and its use for magnetoencephalography forward calculation
in realistic volume conductors. Phys Med Biol, 48(22), 3637-3652.
Noordzij, M. L., Newman-Norlund, S. E., de Ruiter, J. P., Hagoort, P., Levinson, S. C., \& Toni, I. (2009). Brain mechanisms underlying human communication. Frontiers in Human Neuroscience, 3,14

Noordzij, M. L., Newman-Norlund, S. E., de Ruiter, J. P., Hagoort, P., Levinson, S. C., \& Toni, 1. (2010). Neural correlates of intention

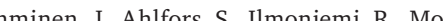

Numm \& Nenonen, J., (1995). Transformation multichannel magnetocardiographic signals to standard grid form. IEEE Trans Biomed Eng, 42(1), 72-78

Nurminen, J., Taulu, S., \& Okada, Y. (2008) Effects of sensor calibration, balancing an parametrization on the signal space separatio method. Phys Med Biol, 53(7), 1975-1987.

O'Neill, M., Bard, K. A., Linnell, M., \& Fluck, M. (2005). Maternal gestures with 20-month-old Shea, J Boudrias M. H. Stagg C. J, 352-359. V., Kischka, U., Blicher, J. U., \& Johansen-Berg, TDCS in chrodicting behavionsal response

kun, M., Naim, A., \& Lampl, I. (2010). The subthreshold relation between cortical loca field potential and neuronal firing unveiled by intracellular recordings in awake rats. Neurosci, 30(12), 4440-4448.

Onton, J., \& Makeig, S. (2009). Highfrequency Broadband Modulations of Electroencephalographic Spectra. Front Hum Neurosci, 3, 61.

Oostenveld, R., Fries, P., Maris, E., \& Schoffelen, J. M. (2011). FieldTrip: Open source software for lectrophysiological data. Comput Intell Neurosci, 2011, 156869.

Orban de Xivry, J. J., Marko, M. K., Pekny, S. E. Pastor, D, Izawa, J., Celnik, P. \& Shadmehr, R. (2011). Stimulation of the human moto cortex alters generalization patterns of moto learning. J Neurosci, 31(19), 7102-7110.

Oshima-Takane, Y. (1988). Children learn from speech not addressed to them: the case of personal pronouns. J Child Lang, 15(1), 95-108.

(1996). Birth Goder effects on early leng, J. L. (1996). Birth order effects on early language from overheard speech? Child Development, 67(2), 621-634.

Owings, D., \& Morton, E. (1998). Animal vocal communication: a new approach. New York: Cambridge University.
Paus, T., Jech, R., Thompson, C. J., Comeau, R., Peters, T., \& Evans, A. C. (1997). Transcranial magnetic stimulation during positron emission tomography: a new method for studying connectivity of the human

Neurosci, 17(9), 3178-3184.
Peelen, M. V., Fei-Fei, L., \& Kastner, S. (2009). Neural mechanisms of rapid natural scene
categorization in human visual cortex. Nature, 460 (7251), 94-97.

Perner, J., Ruffman, T., \& Leekam, S. R. (1994). Theory of Mind Is Contagious - You Catch It from Your
$1228-1238$.

Peterson, C. C. \& Siegal, M. (2000). Insights into theory of mind from deafness and autism. Mind \& Language, 15(1), 123-145.

Pickering, M. J., \& Garrod, S. (2004). Toward mechanistic psychology of dialogue. Behavioral and Brain Sciences, 27(2), 169-190, discussio

190-226.
Pickering, M. J., \& Garrod, S. (2007). Do people use language production to make predictions $105-110$.

Pickering, M. J., \& Garrod, S. (2013). An integrated theory of language production and 36(4), 329-347.

Premack, D., \& Woodruff, G. (1978). Does the Chimpanzee Have a Theory of Mind. Behavioral and Brain Sciences, 1(4), 515-526.

Price, C. J., \& Friston, K. J. (1997). Cognitive conjunction: a new approach to brain activation

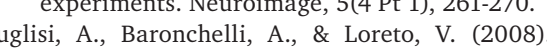
Cultural route to the emergence of linguistic categories. Proc Natl Acad Sci U S A, 105(23), $7936-7940$

Quine, W. V. (1960). Word and Object. Cambridge, Mass: MIT Press.

eod, A. M., Snyder, A. Z. powers, W. J., Gusnard, D. A., \& Shulman, G. L. Natl Acad Sci U S A, 98(2), 676-682.

Raven, J. (1989). The Raven Progressive Matrices a Review of National Norming Studies and Ethnic and Socioeconomic Variation within Measurement, 26(1), 1-16

Raven, J. (2000). The Raven's Progressive Matrices: Cognitive Psychology, 41(1), 1-48.

Ray, S., \& Maunsell, J. H. (2011). Different origins of gamma rhythm and high-gamma activity
in macaque visual cortex. PLoS Biol, 9(4) e1000610
Rizzolatti, G., \& Craighero, L. (2004). The mirrorneuron $169-192$ (2006). Individual differences in theory of mind ability in middle childhood and links with verbal ability and autistic traits. a twi study. Soc Neurosci, 1(3-4), 412-425.

Rorden, C., \& Brett, M. (2000). Stereotaxic display , C. \& Karnath $\mathrm{H}$ O. (2004). Using brain lesions to infer function: a relic from past era in the fMRI age? Nat Rev Neurosci, 5(10), 813-819.

Rubio-Fernandez, P., \& Geurts, B. (2013). How to pass the false-belief task before
birthday. Psychol Sci, 24(1), 27-33

Ruffman, T., Perner, J., Naito, M., Parkin, L., Clements, W. A. (1998). Older (but not younget) siblings facilitate false belief understanding.

Dev Psychol, 34(1), 161-174.
Sabbagh, M. A. (1999). Communicative intentions and language: evidence from right-hemisphere

Sallet, J, Mars, R, B, Noonan, M. P, Andersson, J. L., O'Reilly, J. X., Jbabdi, S Rushworth M. F. (2011). Social network size affects neural circuits in macaques. Science, 334(6056), 697-700.

Sander, T. H., Preusser, J., Mhaskar, R., Kitching J., Trahms, L., \& Knappe, S. (2012). Magnetoencephalography with a chip-scale atomic magnetometer. Biomed Opt Express,

Sass, K., Krach, S., Sachs, O., \& Kircher, T. (2009). Lion - tiger - stripes: Neural correlates of mallities. Neuroimase 45(1), 224-236.

Saxe, R., Xiao, D. K., Kovacs, G Perrett D. I Kanwisher, N. (2004). A region of right posterio superior temporal sulcus responds to observed intentional actions. Neuropsychologia, 42(11), 1435-1446.

Schilbach, L., Timmermans, B., Reddy, V., Costall, A., Bente, G., Schlicht, T., \& Vogeley, A Behavioral and Brain Sciences, 36(4), 393-414

Schippers, M. B., Roebroeck, A., Renken, R, Nanetti, L., \& Keysers, C. (2010). Mapping the during gestural com one brain Acad Sci U S A, 107(20), 9388-9393.

Schultz, J, Friston, K. J, O'Doherty, J, Wolpert, D. M., \& Frith, C. D. (2005). Activation in posterior superior temporal sulcus parallels parameter inducing the percept of animacy. Neuron, 45(4), 625-635. 
Scott-Phillips, T. C., Kirby, S., \& Ritchie, G. R. (2009). Signalling signalhood and the emergence of communication. Cognition, 113(2), 226-233. Searle, S. (2010). Making the Social World: The
Structure of Human Civilization: Oxford Structure of Human

Segaert, K., Menenti, L., Weber, K., Petersson, K. M., \& Hagoort, P. (2012). Shared syntax in language production and language comprehension--an

alten, R, \& Warglien, M. (2007). The emergence of simple languages in an experimental coordination game. Proc Natl Acad Sci U S A, 104(18), 7361-7366.

Shamay-Tsoory, S. G., Aharon-Peretz, J., \& Perry, D. (2009). Two systems for empathy: a double dissociation between emotional and cognitive empathy in inferior frontal gyrus versus ventromedial prefrontal lesions. Brain, 132(Pt 3), 617-627.

Shamay-Tsoory, S. G., Tomer, R., Berger, B. D., Goldsher, D., \& Aharon-Peretz, J. (2005). Impaired "affective theory of mind" is damage. Cogn Behav Neurol, 18(1), 55-67. Shannon, C. (1948). A mathematical theory of
communication. The Bell System Technical Journal, 27, 379-423.

Shatz, M., \& Gelman, R. (1973). The development of communication skills: modifications in the speech of young children as a function of listener. Monogr Soc Res Child Dev, 38(5), $1-38$.

Shultz, S., Lee, S. M., Pelphrey, K., \& McCarthy, G. (2011). The posterior superior temporal sulcus is sensitive to the outcome of human and nonand Affective Neuroscience, 6(5), 602-611

Siegal, M., \& Varley, R. (2002). Neural system involved in "theory of mind". Nat Rev Neurosci, 3(6), 463-471.

Singh, K. D., Holliday, I. E., Furlong, P. L., \& Harding, G. F. (1997). Evaluation of MRI-MEG/ EEG co-registration strategies using Monte Carlo simulation. Electroencephalogr Clin Neurophysiol, 102(2), 81-85,

Smith, S. M. (2002). Fast robust automated brain extraction. Hum Brain Mapp, 17(3), 143-155. Snowden, J. S., Neary, D., \& Mann, D. M. (2002). 140-143.

Sperber, D., \& Wilson, D. (2001). Relevance: communication and cognition: Blackwell Publishers. Steels, L. (2003). Evolving grounded communicatio
for robots. Trends Cogn Sci, 7(7), 308-312.
Stephens, G. J., Silbert, L. J., \& Hasson, U. (2010), Speaker-listener neural coupling underlie successful communication. Proc Natl Acad Sci U S A, 107(32), 14425-14430

Sterzer, P., Haynes, J. D., \& Rees, G. (2006). Primary visual cortex activation on the path of apparen motion is mediated by feedback from hMT + 5. Neuroimage, 32(3), 1308-1316.

Stolk, A., Hunnius, S., Bekkering, H., \& Toni, (2013). Early social experience predicts year-old children. PLos One, 8(8), e72667.

Stolk, A., Todorovic, A., Schoffelen, J. M., \& Oostenveld, R. (2013). Online and offline tools for head movement compensation in MEG. Neuroimage, 68, 39-48.

Stolk, A., Verhagen, L., Schoffelen, J. M., Oostenveld, R., Blokpoel, M., Hagoort, P., ... Toni, 1. (2013). Neural mechanisms of communicative innovation. Proc Natl Acad Sci U S A, 110(36), 14574-14579.

Stone, V. E., Baron-Cohen, S., \& Knight, R. T. (1998) Contal lobe contributions to theory of mind.

dre, G., Parkkonen, L., Bock, E., Baillet, rtMEG: a real-time software interface fo magnetoencephalography. Comput Intell Neurosci, 2011, 327953.

Taulu, S., Simola, J., \& Kajola, M. (2005). Application of the signal space separation method. IEE Transactions on Signal Processing, 53(9), 3359-3372.

Thesen, S., Heid, O., Mueller, E., \& Schad, L. R. (2000). Prospective acquisition correction for head motion with image-based tracking $457-465$.

Todorovic, A., \& de Lange, F. P. (2012). Repetition suppression and expectation are dissociable in time in early auditory evoked fields. J Neurosci, In press.

. P. (2011). Prior expectation mediates neural adaptation to repeated sounds in the auditory cortex: an MEG study. J Neurosci, 31(25), 9118-9123.

Tognoli, E., Lagarde, J., DeGuzman, G. C., \& Kelso, J. A. (2007). The phi complex as a neuromarke Sci U S A, 104(19), 8190-8195.

Tomasello, M. (2008). Origins of human communication. Cambridge, Mass.: MIT Press. Townsend, J. T., \& Ashby, F. G. (1983). Stochastic Modeling of Elementary Psychological
Processes. Cambridge [Cambridgeshire] Cambridge University Press.
Tranel, D., Bechara, A., \& Denburg, N. L. (2002). Asymmetric functional roles of right and left ventromedial prefrontal cortices in social conduct, decision-making, and

processing. Cortex, 38(4), 589-612.
.

Thesson, H. K., \& Ghazanfar, A. A. (2011). Statistical learning of social signals and its implication for the social brain

Tzourio-Mazoyer, N., Landeau, B., Papathanassiou, D., Crivello, F., Etard, O., Delcroix, N., . . Joliot, M. (2002). Automated anatomical labeling of activations in SPM using a macroscopic anatomical parcellation of the MNI MRI singlesubject brain. Neuroimage, 15(1), 273-289.

Uutela, K., Taulu, S., \& Hamalainen, M. (2001). Detecting and correcting for head movements in neuromagnetic measurements. Neuroimage, 14(6), 1424-1431

Valero-Cabre, A., Payne, B. R., \& Pascual-Leone, A. (2007). Opposite impact on (14)C-2 deoxyglucose brain metabolism following patterns of high and low frequency repetitive posterior parietal cortex. Experimental Brain Research, 176(4), 603-615.

van Ackeren, M. J. Casasanto, D., Bekkering, H. Hagoort, P., \& Rueschemeyer, S. A. (2012). Pragmatics in action: indirect requests engage theory of mind areas and the cortical moto network. J Cogn Neurosci, 24(1), 2237-2247.

van Berkum, J. J., van den Brink, D., Tesink, C. M. Kos, M., \& Hagoort, P. (2008). The neural integration of speaker and message. $J$ Cogn

Van IJzendoorn, M. H., Sagi, A., \& Lambermon, M. Data from Holland and Israel. In R. C. Piant (Ed.), Beyond the Parent: The Role of Othe Adults in Children's Lives. (pp, 5-24) San Francisco: Voland, Eckar and Jan Biese.

van Pelt, S., Boomsma, D. I., \& Fries, P. (2012). Magnetoencephalography in twins reveals a strong genetic determination of the peak frequency of visually induced gamma-
band synchronization. J Neurosci, 32(10), band synchro
3388-3392.

van Rooij, I. Kwisthout, J., Blokpoel, M., Szymanik, J., Wareham, T., \& Toni, I. (2011). Intentional difficult? Frontiers in Human Neuroscience, 5 ,

Van Veen, B. D., van Drongelen, W., Yuchtman, M. \& Suzuki, A. (1997). Localization of brain electrical activity via linearly constrained minimum variance spatial filtering. IEEE Tran Biomed Eng, 44(9), 867-880.
Verhagen, L., Dijkerman, H. C., Medendorp, W. P., \& Toni, I. (2012). Cortical dynamics of sensorimotor integration during gras planning. J Neurosci, 32(13), 4508-4519.

Volman, I., Noordzij, M. L., \& Toni, I. (2012). Sources of variability in human communicative skills. Front Hum Neurosci, 6, 310

Walter, H., Adenzato, M., Ciaramidaro, A., Enrici, .., Aa, L., \& Bara, B. G. (2004). Understanding (16)rior paracingulate cortex. J Cogn Nemrosi, 16(10), 1854-1863.

Warrenleubecker, A, \& Bohannon, J. N. (1984) Intonation Patterns in Child-Directed Speech Mother-Father Differences. Child Development, 55(4), 1379-1385.

Weder, N. D., Aziz, R., Wilkins, K., \& Tampi, R. R. (2007). Frontotemporal dementias: a review. Ann Gen Psychiatry, 6, 15

Wehner, D. T., Hamalainen, M. S., Mody, M., \& Ahlfors, S. P. (2008). Head movements of children in MEG: quantification, effects Neuroimage, 40(2), 541-550.

Wellman, H. M. Cross, D, \& Watson, J (2001) Meta-analysis of theory-of-mind development: the truth about false belief. Child Development 72(3), 655-684

Willems, R. M., de Boer, M., de Ruiter, J. P. Noordzij, M. L., Hagoort, P., \& Toni, I. (2010). A Dissociation Between Linguistic and Communicative Abilities in the Human Brain. Psychological Science, 21(1), 8-14

Wilson, H. S. (2004). Continuous head-localization and data correction in a whole-cortex ME sensor. Neurol Clin Neurophysiol, 2004, 56 .
Wittgenstein, L. (1953/2001). Philosophical Investigations: Blackwell Publishing

Woltz, D. J., \& Was, C. A. (2006). Availability of related long-term memory during and after attention focus in work
Cognit, 34(3), 668-684

Worsley, K. J., \& Friston, K. J. (1995). Analysis of fMRI time-series B. C., Hudac, C. M., Carter, E. J., Sobel, D. M., \& Pelphrey, K. A. (2009). Action understanding in the superior temporal sulcus region

Young, L., Camprodon, J. A., Hauser, M., Pascualof the right temporoparietal junction with transcranial magnetic stimulation reduces the role of beliefs in moral judgments. Proc Nat Acad Sci U S A, 107(15), 6753-6758 


\section{Donders Graduate School \\ for Cognitive Neuroscience Series}

van Aalderen-Smeets, S.I. (2007). Neural dynamics of visual selection. Maastrich dynamics of visual selection. Maas
University, Maastricht, the Netherlands.

2. Schoffelen, J.M. (2007). Neuronal communication through coherence in the huma
motor system. Radboud University Nijmegen Nijmegen, the Netherlands.

3. de Lange, F.P. (2008). Neural mechanisms of motor imagery. Radboud University Nijmegen, Nijmegen, the Netherlands.

4. Grol, M.J. (2008). Parieto-frontal circuitry in visuomotor control. Utrecht University, Utrech the Netherlands.

Bauer, M. (2008). Functional roles of rhythmic neuronal activity in the human visual and Nijmegen, Nijmegen, the Netherlands.

6. Mazaheri, A. (2008). The Influence of Ongoing Oscillatory Brain Activity on Evoked Responses and Behaviour. Radboud University Nijmegen, Nijmegen, the Netherlands.

7. Hooijmans, C.R. (2008). Impact of nutritional lipids and vascular factors in Alzheimer's Disease. Radboud University Nijmegen,

8. Gaszner, B. (2008). Plastic responses to stress by the rodent urocortinergic Edinger-Westphal Nijmegen, the Netherlands.

9. Willems, R.M. (2009). Neural reflections of meaning in gesture, language and action. Radboud University Nijmegen, Nijmegen, the Netherlands.

10. van Pelt, S. (2009). Dynamic neural representations of human visuomotor space. Radboud University Nijmegen, Nijmegen, the Netherlands.
11. Lommertzen, J. (2009). Visuomotor coupling at different levels of complexity. Radboud University Nijmegen, Nijmegen, the Netherlands.

12. Poljac, E. (2009). Dynamics of cognitive control in task switching: Looking beyond the switch cost. Radboud University Nijmegen, Nijmegen, the Netherlands.

13. Poser, B.A. (2009). Techniques for BOLD and blood volume weighted fMRI. Radboud University Nijmegen, Nijmegen, the

14. Baggio, G. (2009). Semantics and the electrophysiology of meaning. Tense, aspect,

van Wingen, G.A. (2009). Biological determinants of amygdala functioning. Radboud University Nijmegen Medical Centre, Nijmegen, the Netherlands.

16. Bakker, M. (2009). Supraspinal control of walking: lessons from motor imagery. Radboud University Nijmegen Medical Centre, Nijmegen,

17. Aarts, E. (2009). Resisting temptation: the role of the anterior cingulate cortex in adjusting cognitive control. Radboud Universily

18. Prinz, S. (2009). Waterbath stunning of the electroencephalogram and physical reflexes of broilers. Radboud University Nijmegen, Nijmegen, the Netherlands.

19. Knippenberg, J.M.J. (2009). The N150 of the Auditory Evoked Potential from the rat amygdala. In search for its functional significance. Radboud University Nijmegen, Nijmegen, the Netherlands.
20. Dumont, G.J.H. (2009). Cognitive and physiological effects of 3,4-methylenedioxymethamphetamine (MDMA or 'ecstasy') in combination with alcohol or cannabis in humans. Radboud

21. Pijnacker, J. (2010). Defeasible inference in autism: a behavioral and electrophysiogical Nijmegen, the Netherhis

22. de Vrijer, M. (2010). in spatial orientation. Radboud University Nijmegen, Nijmegen, the Netherlands.

23. Vergeer, M. (2010). Perceptual visibility and appearance: Effects of color and form. Radboud University Nijmegen, Nijmegen, the Netherlands.

24. Levy, J. (2010). In Cerebro Unveiling Unconscious Mechanisms during Reading. Radboud University Nijmegen, Nijmegen, the Netherlands.

25. Treder, M. S. (2010). Symmetry in (inter)action. Nijmegen, Nijmegen, the Netherlands.

26. Horlings, C.G.C. (2010). A Weak balance; balance and falls in patients with neuromuscula disorders. Radboud University Nijmegen, Nijmegen, the Netherlands.

27. Snaphaan, L.J.A.E. (2010). Epidemiology of post-stroke behavioural consequences. Radboud University Nijmegen Medical Centre, Nijmegen, the Netherlands.

28. Dado - van Beek, H.E.A. (2010). The regulation of cerebral perfusion in patients with Alzheimer's disease. Radboud University Nijmegen Medical
Centre, Nijmegen, the Netherlands. Centre, Nimegen, the Netherlands.

29. Derks, Nime nonpregangenendent stress adaptation in rodents. Radboud University Nijmegen, Nijmegen, the Netherlands.

30. Wyczesany, M. (2010). Covariation of mood and brain activity. Integration of subjective self-report data with quantitative EEG measures. Radboud University Nijmegen, Nijmegen, the Netherlands.

31. Beurze, S.M. (2010). Cortical mechanisms for reach planning. Radboud University Nijmegen, Nijmegen, the Nethelands.

2. van the Netherlands.

33. Lapatki, B.G. (2010). The Facial Musculature - Characterization at a Motor Unit Level. Radboud University Nijmegen, Nijmegen, the Netherlands.
4. Kok, P. (2010). Word Order and Verb Inflection in Agrammatic Sentence Production. Netherlands.

35. van Elk, M. (2010). Action semantics: Functional and neural dynamics. Radboud University

Nijmegen, Nijmegen, the Netherlands.
Majdandzic, J. (2010). Cerebral mechanisms frothers

7. Snijers, T.M.

M. (2010). More than words - neural

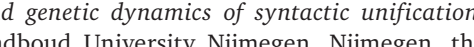
Netherlands.

38. Grootens, K.P. (2010). Cognitive dysfunction and effects of antipsychotics in schizophren and borderline personality disorder. Radboud University Nijmegen Medical Centre, Nijmegen, the Netherlands.

9. Nieuwenhuis, I.L.C. (2010). Memory consolidation: A process of integration bonverging evidence from MEG, FMRI and Medical Centre, Nijmegen, the Netherlands.

40. Menenti, L.M.E. (2010). The right language: differential hemispheric contributions to language production and comprehensio in context. Radboud University Nijmegen, Nijmegen, the Netherlands.

41. van Dijk, H.P. (2010). The state of the brain how alpha oscillations shape behaviour and event related responses. Radboud University Nijmegen, Nigmegen, the Netherlands.

. Meulenbrek, O.N. (2010). Neural correlates of episodic memory in healthy aging an Nijmegen, Nijmegen, the Netherlands.

43. Oude Nijhuis, L.B. (2010). Modulation of Nijmegen, Nijmegen, the Netherlands.

44. Qin, S. (2010). Adaptive memory: imaging medial temporal and prefrontal memory system Radboud Un
Netherlands.

45. Timmer, N.M. (2011). The interaction of hepara sulfate proteoglycans with the amyloid protein. Radboud University Nijnegen, Nijmegen, the

Craje, C. (2011). (A)typical motor planning and . Radboud University Nijmegen, the Netherlands.

47. van Grootel, T J. (2011). On the role of eye and head position in spatial localisation behaviour. Radboud University Nijmegen, Nijmegen, the Netherlands. 
48. Lamers, M.J.M. (2011). Levels of selective attention in action planning. Radboud U

Nijmegen, Nijmegen, the Netherlands.

9. van der Werf, J. (2011). Cortical oscillatory activity in human visuomotor integration. Radboud University Nijmegen, Nijmegen, the Netherlands.

50. Scheeringa, R. (2011). On the relation between oscillatory EEG activity and the BOLD stgnal. Radboud Univers

1. Bögels, S. (2011). The role of prosody in language comprehension: when prosodic breaks and pitch Nijmegen, Nijmegen, the Netherlands.

52. Ossewaarde, L. (2011). The mood cycle: hormonal influences on the female brain Radboud University Nijmegen, Nijmegen, the Netherlands.

53. Kuribara, M. (2011). Environment-induced activation and growth of pituitary melanotrope cells of Xenopus laevis. Radboud University

4. Helmich, R.C. (2011) Cerbot

in Parkinson's disease. Radboud Univation Nijmegen, Nijmegen, the Netherlands.

. Boelen, D. (2011). Order out of

Assessment and treatment of executive disorders in brain-injured patients. Radboud University Nijmegen, Nijmegen, the Netherlands.

56. Koopmans, P.J. (2011). fMRI of cortical layers. Radboud University Nijmegen, Nijmegen, the

Netherlands.
57. van der Linden, M.H. (2011). Experience based cortical plasticity in object category Nijmegen, the Netherlands.

58. Kleine, B.U. (2011). Motor unit discharges Physiological and diagnostic studies in AIS Radboud University Nijmegen Medical Centre, Nijmegen, the Netherlands.

59. Paulus, M. (2011). Development of action perception: Neurocognitive mechanisms underlying children's processing of others' actions. Radboud University Nijmegen, Nijmegen, the Netherlands.

60. Tieleman, A.A. (2011). Myotonic dystrophy type 2. A newly diagnosed disease in the Netherlands. Radboud University Nijmegen Medical Centre

van Leeuwen T M. (2011)

is not there'. Newral (2011). 'How one can see what is not there. Neural mechanisms of graphemeNijmegen, Nijmegen, the Netherlands.
62. van Tilborg, I.A.D.A. (2011). Procedural learning in cognitively impaired patients and its application in clinical practice. Radboud University Nijmegen, Nijmegen, the Netherlands.

63. Bruinsma, I.B. (2011). Amyloidogenic protein in Alzheimer's disease and Parkinson's disease interaction with chaperones and inflammation. Netherlands. Ehlers-Danlos syndrome and Marfan syndrome; expanding the phenotype of inherited connective tissue disorders and investigating the role of the extracellular matrix in muscle. Radboud University Nijmegen Medical Centre, Nijmegen, the Netherlands.

65. Reelick, M. (2011). One step at a time Disentangling the complexity of preventing falls in frail older persons. Radboud University Nijmegen Medical Centre, Nijmegen, the Netherlands.

66. Buur, P.F. (2011). Imaging in motion University Nijmegen, Nijmegen, the Netherlands.

67. Schaefer, R.S. (2011). Measuring the mind's ear: EEG of music imagery. Radboud University Nijmegen, Nijmegen, the Netherlands.

68. Xu, L. (2011). The non-preganglionic EdingerWestphal nucleus: an integration center for energy balance and stress adaptation Radboud University Nijmegen, Nijmegen, the Netherlands.

69. Schellekens, A.F.A. (2011). Gene-environmen interaction and intermediate phenotypes in
alcohol dependence. Radboud University Nijmon Nijmegen the Netherlonds.

van Marle, HJF. (2011) The amydala on

A neuroimaging investigation into on alert: function during acute stress and its aftermath. Radboud University Nijmegen, Nijmegen, the Netherlands.

71. de Laat, K.F. (2011). Motor performance in individuals with cerebral small vessel disease: an MRI study. Radboud University Nijmege Medical Centre, Nijmegen, the Neth

72. Madebach, A. (2011). Lexical access in speaking Studies on lexical selection and cascading Nijmegen, the Netherlands.

Poelmans, G JV. (2011).

networks for neurodevelopen and protein Radboud University Nijmegen, Nijmegen, the Netherlands.
74. van Norden, A.G.W. (2011). Cognitive function in elderly individuals with cerebral small vessel disease. An MRI study. Radboud University Nijmegen Medical Centre, Nijmegen, the Netherlands.

75. Jansen, E.J.R. (2011). New insights into $V$-ATPase functioning: the role of its accessory subunit Ac45 and a novel brain-specific Ac45 paralog. Radboud University Nijmegen,
Nijmegen, the Netherlands.

6. Haaxma, C.A. (2011). New perspectives on Radboud University Nijmegen Medical Centre, Nijmegen, the Netherlands.

77. Haegens, S. (2012). On the functional role of oscillatory neuronal activity in the somatosensory system. Radboud University Nijmegen, Nijmegen, the Netherlands.

78. van Barneveld, D.C.P.B.M. (2012). Integration of exteroceptive and interoceptive cues in spatial localization. Radboud University Nijmegen

79. Spies, P.E. (2012). The reflection of Alzheimer Tedice

. Helle, M. (2012). Artery-specific perfusion measurements in the cerebral vasculature by magnetic resonance imaging. University Nijmegen, Nijmegen, the Netherlands

81. Egetemeir, J. (2012). Neural correlates of reallife joint action. Radboud University Nijmegen, Nijmegen, the Netherlands.

82. Janssen, L. (2012). Planning and execution of (bi)manual grasping. Radboud University Nijmegen, Nijmegen, the Netherlands.

83. Vermeer, S. (2012). Clinical and genetic characterisation of Autosomal Recessive
Cerebellar Ataxias. Radboud University Nijmegen Medical Centre, Nijmegen, the Netherlands.

84. Vrins, S. (2012). Shaping object boundaries contextual effects in infants and adults. Radboud University Nijmegen, Nijmegen, the Netherlands.

85. Weber, K.M. (2012). The language learning brain: Evidence from second language and bilingual studies of syntactic processing. Netherlands.

Unagen, L. (2012). How to grasp a ripe tomato.

Nonkes, L J P. (2012) Sertonin transporter gene variance causes individual differences in rat behaviour: For better and for worse. Radboud University Nijmegen Medical Centre, Nijmegen, theNetherlands.
8. Joosten-Weyn Banningh, L.W.A. (2012) Learning to live with Mild Cognitive Impairmen: development and evaluation of a psychologica intervention for patients with Mild Cognitive Impairment and their significant others. Radboud University Nijmegen Med Xjmegen, the Netherlands.

89. Xiang, HD. (2012). The language network of

Snijders, A.H. (2012). Tackling freezing of gait Nijmegen Medical Centre, Nijmegen, the Netherlands.

91. Rouwette, T.P.H. (2012). Neuropathic pain and the brain - Differential involvement of corticotropinreleasing factor and urocortin in acute and chronic pain processing. Radboud University Nijmegen Medical Centre, Nijmegen, the Netherlands.

. Van de Meerendonk, N. (2012). States of indecision in the brain: Electrophysiological visual language perception. Radboud University Nijmegen, Nijmegen, the Netherlands.

93. Sterrenburg, A. (2012). The stress response of forebrain and midbrain regions: Neuropeptides sex-specificity and epigenetics. Radbou University Nijmegen, Nijmegen, The Netherlands.

94. Uithol, S. (2012). Representing action and intention. Radboud University Nijmegen,

5. Van Dam, W.O. (2012). On the specificity and flexibility of embodied lexical-semantic representations. Radboud University Nijmegen,
Nijmegen, The Netherlands.

96. Slats, D. (2012). CSF biomarkers of Alzheimer's disease: Serial sampling analysis and the study of circadian rhythmicity. Radboud University Nijmegen M
Netherlands.

97. Van Nuenen, B.F.L. (2012). Cerebral reorganization in premotor parkinsonism. Radboud University Nijmegen Medical Centre, Nijmegen, the Netherlands.

8. van Schouwenburg, M.R. (2012). Frontostriatal mechanisms of attentional control. Radboud University Nijmegen, Nijmegen, The - Azar, M.G. (2012

Aarning. (2012). On the theory of reinforcemen and sample complexity. Radboud University Nijmegen, Nijmegen, The Netherlands.

100. Meeuwissen, E.B. (2012) Cortical oscillatory activity during memory formation. Radboud University Nijmegen, Nijmegen, The Netherlands. 
101. Arnold, J.F. (2012). When mood meets memory: Neural and behavioral perspectives on emotional memory in health and depression. Radboud University Nijmegen, Nijmegen, The Netherlands.

102. Gons, R.A.R. (2012). Vascular risk factors in cerebral small vessel disease: A diffusion tensor imaging study. Radboud University Nijmege

203. Medical Centre, Nijmegen, the Netherlands.

03. Wingbermahle, E. (2012). Cognition and A neuropsychological perspective. Radboud University Nijmegen, Nijmegen, The Netherlands.

104. Walentowska, W. (2012). Facing emotional faces. The nature of automaticity of facial emotion processing studied with ERPS. Radboud University Nijmegen, Nijmegen, The Netherlands.

105. Hoogman, M. (2012). Imaging the effects of ADHD risk genes. Radboud University Nijmegen, Nijmegen, The Netherlands.

106. Tramper, J.J. (2012). Feedforward and feedback mechanisms in sensory motor control. Netherlands.

107. Van Eijndhoven, P. (2012). State and trait characteristics of early course major depressive
disorder. Radboud University Nijmegen Medical Centre, Nijmegen, the Netherlands.

108. Visser, E. (2012). Leaves and forests: Low level sound processing and methods for the large-scale analysis of white matter structure in autism. Radboud University Nijmegen, Nijmegen, The Netherlands.

109. Van Tooren-Hoogenboom, N. (2012). Neuronal communication in the synchronized brain. induced gamma band activity: Lessons from $M E G$. Radboud University Nijmegen, Nijmegen, The Netherlands.

110. Henckens, M.J.A.G. (2012). Imaging the stressed brain. Elucidating the time- and region-specific effects of stress hormones on brain function: A translational approach. Radboud University Nijmegen, Nijmegen, The Netherlands.

111. Van Kesteren, M.T.R. (2012). Schemas in the brain: Influences of prior knowledge on learning,memory, and education. Radboud Netherlands

112. Brenders, P. (2012). Cross-language interactions in beginning second language learners. Radboud University Nijmegen, Nijmegen, The Netherlands.
113. Ter Horst, A.C. (2012). Modulating motor imagery. Contextual, spatial and kinaesthetic influences. Radboud University Nijmegen, Nijmegen, The Netherlands.

114. Tesink, C.M.J.Y. (2013). Neurobiological insights into language comprehension in autism Context matters. Radboud University Nijmegen, Nijmegen, The Netherlands.

115. Böckler, A. (2013). Looking at the world together. How others' attentional relations to jointly Radboud University Nijmegen, Nijmegen, The Netherlands.

116. Van Dongen, E.V. (2013). Sleeping to Remember. On the neural and behavioral mechanism of sleep-dependent memory consolidation. Radboud University Nijmegen, Nijmegen, The Netherlands.

117. Volman, I. (2013). The neural and endocrin regulation of emotional actions. Radboud University Nijmegen, Nijmegen, The Netherlands.

118. Buchholz, V. (2013). Oscillatory activity Nijmegen, Nijmegen, The Netherlands.

19. Van Deurzen, P. A.M (2013), In for

processing and depressive symptoms in healthy adolescents. Radboud University Nijmegen, Nijmegen, The Netherlands.

120. Whitmarsh, S. (2013). Nonreactivity an metacognition in mindfulness. Radboud University Nijmegen, Nijmegen, The Netherlands.

121. Vesper, C. (2013). Acting together: Mechanism of intentional coordination. Radboud University Nijmegen, Nijmegen, The Netherlands.

122. Lagro, J. (2013). Cardiovascular and in clinical practice and prognostics in geriatric patients. Radboud University Nijmegen Medica Centre, Nijmegen, the Netherlands.

123. Eskenazi, T.T. (2013). You, us \& them: from motor simulation to ascribed shared intentionality in social perception. Radboud University Nijmegen, Nijmegen, The Netherlands

124. Ondobaka, S. (2013). On the conceptual and perceptual processing of own and others behavior. Radboud University Nijmegen, Nimnegen, The Netherlands.

Orestic acquisition of movement sequences to identify the best learning condition in good and poor writers. Radboud University Nijmegen, Nijmegen, The Netherlands.
126. Kalisvaart, J.P. (2013). Visual ambiguity in perception and action. Radboud University
Nijmegen Medical Centre, Nijmegen, The Netherlands

127. Kroes, M. (2013). Altering memories for emotional experiences. Radboud University Nijmegen, Nijmegen, The Netherlands

128. Duijnhouwer, J. (2013). Studies on the rotation problem in self-motion perception. Netherlands.

Netherlands.

(2013). Macroscopic networks in the human brain: Mapping connectivity in healthy and damaged brains. Univ
Twente, Enschede, The Netherlands.

130. Braakman, M.H. (2013). Posttraumatic stres disorder with secondary psychotic features. A diagnostic validity study among refugees in the Netherlands. Radboud University Nijmegen, Nijmegen, The Netherlands.

131. Zedlitz, A.M.E.E. (2013). Brittle brain power. Post-stroke fatigue, explorations into assessment Nijmegen,

132. Schoon, Y (2013), From a gait and falls clinic visit towards self-management of falls in frail elderly. Radboud University Nijmegen Medica Centre, Nijmegen, The Netherlands.

133. Jansen, D. (2013). The role of nutrition in Alzheimer's disease - A study in transgenic mouse models for Alzheimer's disease and vascular disorders. Radboud University Nijmegen,

134. Kos, M. (2013). On the waves of language Electrophysiological reflections on semantic and syntactic processing. Radboud Univ
Nijmegen, Nijmegen, The Netherlands. 135. Severens, M. (2013). Towards clinical BCI rehabilitation. Radboud University Nijmegen, Nijmegen, Sint Maartenskliniek, Nijmegen, The Netherlands.

136. Bergmann, H. (2014). Two is not always better than one: On the functional and neural (in) dependence of working memory and long-term memory. Radboud University Nijmegen, Nijmegen, The Netherlands.

137. Wronka, E. (2013). Searching for the biological basis of human mental abilitites. The tudied with P3. Radboud University Nijnegen, Nijmegen, The Netherlands.

thalamo-cortical system in role of the corticoRadboud University Nijmegen, Nijmegen, The Netherlands.
139. Brazil, I.A. (2013). Change doesn't come easy: Dynamics of adaptive behavior in psychopathy. Radboud Univers

140. Zerbi, V. (2013). Impact of nutrition on brain structure and function. A magnetic resonance imaging approach in Alzheimer mouse model. Radboud University Nijmegen, Nijmegen, Th

41. Delnooz, C.C.S. (2014). Unravelling primary focal dystonia. A treatment update and
new pathophysiological insights. Radboud University Nijmegen Medical Centre, Nijmegen, The Netherlands.

142. Bultena, S.S. (2013). Bilingual processing of cognates and language switches in sentence context. Radboud University Nijmegen, Nijmegen, The Netherlands.

143. Janssen, G. (2014). Diagnostic assessment of psychiatric patients: A contextual perspective on executive functioning. Radboud University Nijnegen, Nijmegen, The Netherlands.

44. Piai, V. Magalhães (2014). Choosing our of attention in spoken word production. Radboud University Nijmegen, Nijmegen, The Netherlands.

145. Van Ede, F. (2014). Preparing for perception. O the attentional modulation, perceptual relevance and physiology of oscillatory neural activity. Radboud University Nijmegen, Nijmegen, The Netherlands.

46. Brandmeyer, A. (2014). Auditory perceptual learning via decoded EEG neurofeedback: novel paradigm. Radboud University Nijmegen

47. Radke, S. (2014). Acting social: Neuroendocrine and clinical modulations of approach and
decision behavior. Radboud University Nijmegen, Nijmegen, The Netherlands.

148. Simanova, I. (2014). In search of conceptual representations in the brain: towards mindNijmegen, The Netherlands.

149. Kok, P. (2014). On the role of expectation visual perception: A top-down view of early visual cortex. Radboud University Nijmegen,

0. Van Geldorp, B. (2014). The long and the short of memory: Neuropsychological studies on the memory formation. Radboud University Nijmegen, Nijmegen, The Netherlands.

151. Meyer, M. (2014). The developing brain in action - Individual and joint action processin Radboud University Nijmegen, Nijmegen, Th Netherlands. 
152. Wester, A. (2014). Assessment of everyday memory in patients with alcohol-related cognitive disorders using the Rivermead
Behavioural Memory Test. Radboud University Nijmegen, Nijmegen, The Netherlands.

153. Koenraadt, K. (2014). Shedding light on cortical control of movement. Radboud University Nijmegen, Nijmegen; Sint Maartenskliniek, Nijmegen, The Netherlands.

54. Rutten-Jacobs, L.C.A. (2014). Long-term University Nijmegen Medical Centre, Nijmegen, The Netherlands. 155. Herbert, M.K. (2014). Facing uncertain
diagnosis: the use of CSF biomarkers for the differential diagnosis of neurodegenerative diseases. Radboud University Nijmegen Medical Centre, Nijmegen, The Netherlands.

156. Llera Arenas, A. (2014). Adapting brain computer interfaces for non-stationary changes. Radboud University Nijmegen, Nijmegen, The Netherlands.

57. Smulders, K. (2014). Cognitive control of gait and balance in patients with chronic stroke Nijmegen Medical Centre, Nijmegen, The Netherlands.

158. Boyacioglu, R. (2014). On the application of ultra-fast $f M R I$ and high resolution multiband fMRI at high static field strengths. Radboud University Nijmegen, Nijmegen, The Netherlands.

159. Kleinnijenhuis, M. (2014). Imaging fibres in the brain. Radboud University Nijmegen, Nijmegen, The Netherlands.

160. Geuze, J. (2014). Brain Computer Interfaces for Communication: Moving beyond the Nijmegen, The Netherlands.

Platonov, A. (2014). Mech

motion rivalry. Radboud University bijocular Nijmegen, The Netherlands.

162. Van der Schaaf, M.E. (2014). Dopaminergic modulation of reward and punishment learning. Radboud University Nijmegen Medical Centre, Nijmegen, The Netherlands.

163. Aerts, M.B. (2014). Improving diagnostic accuracy in parkinsonism. Radboud University Nijmegen Medical Centre, Nijmegen, The 64. Vlek

40. paradigm for, and the ethical aspects of BrainNijmegen, Nijmegen, The Netherlands.

165. Massoudi, R. (2014). Interaction of task-related and acoustic signals in single neurons of monkey auditory cortex. Radboud University Nijmegen, Nijmegen, The Netherlands
166. Stolk, A. (2014). On the generation of shared symbols. Radboud University Nijmegen, Nijmegen, The Netherlands. 


\section{Acknowledgments}

Analogous to our communicative abilities, the mechanisms supporting the construction of the present work are ill-defined, and certainly cannot be taken out of the social context (e.g. see chapter 5.1). Although the size of this booklet may suggest otherwise, my writing tends to be concise, potentially interfering with the expression of my gratitude in the most important section of the thesis. At risk of missing out on names, here is an attempt for a rough and ready list.

Without the impact of four individuals, this thesis would have turned out significantly different. Ivan Toni, 'il padrino'; science with you is like assembling an Italian motorcycle with one's best friend, not having access to a workshop manual, and thus not knowing whether you will end up with a Ducati, or even better, an Aprilia (without sidecar...). And like riding, the journey itself is no less fun than reaching its destination, as one discusses engineering and improvements thereof en route. Lennart Verhagen; despite that we have never planned a meeting, people seem to have strongly associated us, usually in the context of drinking coffee in 'my office' (some call it 'the library'). And despite what they say about too much caffeine, this work ostensibly has not suffered from our numerous inspiring interactions. Unfortunately I can no longer return you the 'favour' of being my paranymph. Robert Oostenveld \& Jan-Mathijs Schoffelen, a.k.a. 'the general' and 'captain' (sorry for that); conceptually your contributions to this booklet are not negligible. Whether during fantasizing about innovations of electrophysiology (e.g. see chapter 2.1) and other methodological advances that benefit a large scientific community, or when simply 'squashing bugs', I have learnt from each of you. I consider myself not only a co-developer, but also, in part, a product of FieldTrip.

The Donders is a fascinating environment, affording vast potential for (personal) development. Not a day I have left home with reluctance (Frisian understatement again), thanks to a large number of people, other than already mentioned above. For instance, I owe much to the Intention \& Action group; Frank Leone, Sasha Ondobaka, Inge Volman, Lennart Verhagen, Egbert Hartstra, Loek Brinkman, Anke Marit Albers, Marieke van der Schaaf, Miriam de Boer, Florian Krause, Mark Blokpoel, Iris van Rooij, Ivan Toni, Idil Kokal, Marius Zimmermann, Murielle Ferraye, Anke Murillo Oosterwijk, and Ian Cameron for their ongoing support despite my group meeting bombardments on human communication, electrophysiology, and other 'general issues'. The FieldTrip core development team; Robert Oostenveld, Jan-Mathijs Schoffelen, Saskia Haegens, Stephen Whitmarsh, Roemer van der Meij, Jörn Horschig, Eelke Spaak, Diego Lozano Soldevilla, Johanna Zumer, Lilla Magyari, Jim Herring, Nietzsche Lam, and
Haiteng Jiang for jointly building a platform that goes far beyond the level of code. Spending casual time with these scientific enthusiasts, who despite their own projects free up some time to facilitate overall research at the Donders and beyond, is attitude shaping. The Donders soccer team; Eelco van Dongen, Sash Ondobaka, Rene Scheeringa, Peter Koopmans, Tom Marshall, Ruud Berkers, Sean Fallon, Amin Aminaei, Roohollah Massoudi, Rasim Boyacioglu, Egbert Hartstra, Markus Barth, Peter Kok, and Alan Sanfey for dodging cannon balls and bodychecks on thursday evenings. My office mates; Esther Meeuwissen, Marieke van der Schaaf, Ruud Berkers, Tim van Mourik, and Maarten Leenders, for involuntary participation in my conspiracies. My paranymphs; Ruud Berkers \& Loek Brinkman, for closing the gap between Limburg and Friesland and sharing passion for local field potentials and pimpplots. Then there is a number of individuals whose suppport has been so natural that it is easily taken for granted. For instance, Marek Tyc, Edward Gerrits, Rene de Bruin, Bram Daams, Erik van den Boogert, Uriël Plones, Paul Gaalman, Sander Berends, Tildie Stijns, Nicole Stekkinger, Sandra Heemskerk, Joost van Meurs, and Arthur Willemsen. Finally, I owe thanks to a few that I have not mentioned yet given that they could not easily be pigeonholed, but who have contributed, whether directly or indirectly, to the positive, collaborative atmostphere that I have experienced at the Donders; Peter Hagoort, David Norris, Hanneke den Ouden, Floris de Lange, Ana Todorovic, Harold Bekkering, Sabine Hunnius, Larry O’Dwyer, Joost Wegman, Marlieke van Kesteren, Jeanette Mostert, Martine van Schouwenburg, et al. [insert(intranet/telephonelist)] - reached my project quotum.

I have also had the privilege to be surrounded by wonderful individuals outside the Donders. A small selection follows. My family; Ingrid Jurg, Kitty Stolk, Els \& Marianne Jurg, Loes \& Bertus Jurg, Gerrit \& Margriet Stolk, Jos \& Anja Stolk, and Hanneke \& Gerrit Bijl for providing a warm and, especially fun and inspiring nest. My late grandfather; Bertus Jurg, for unwittingly being a role model. My scientific uncle; Bert Otten, for bootstrapping my passion for neural and evolutionary mechanisms. My friends; Viktor Hennink, Martin Koopmans, Youri Kruse, Mark Noordenbos, Lourens Boersma, Wobbe Dijkstra, and others, that despite occasionally large geographical distances remain in contact, or in mind. Last but not least, I would like to thank my biggest support, my better half, and my toughest reviewer. Loes Bijl; the past four years in Nijmegen with you flew by so fast. I hope to share more adventures with you. 


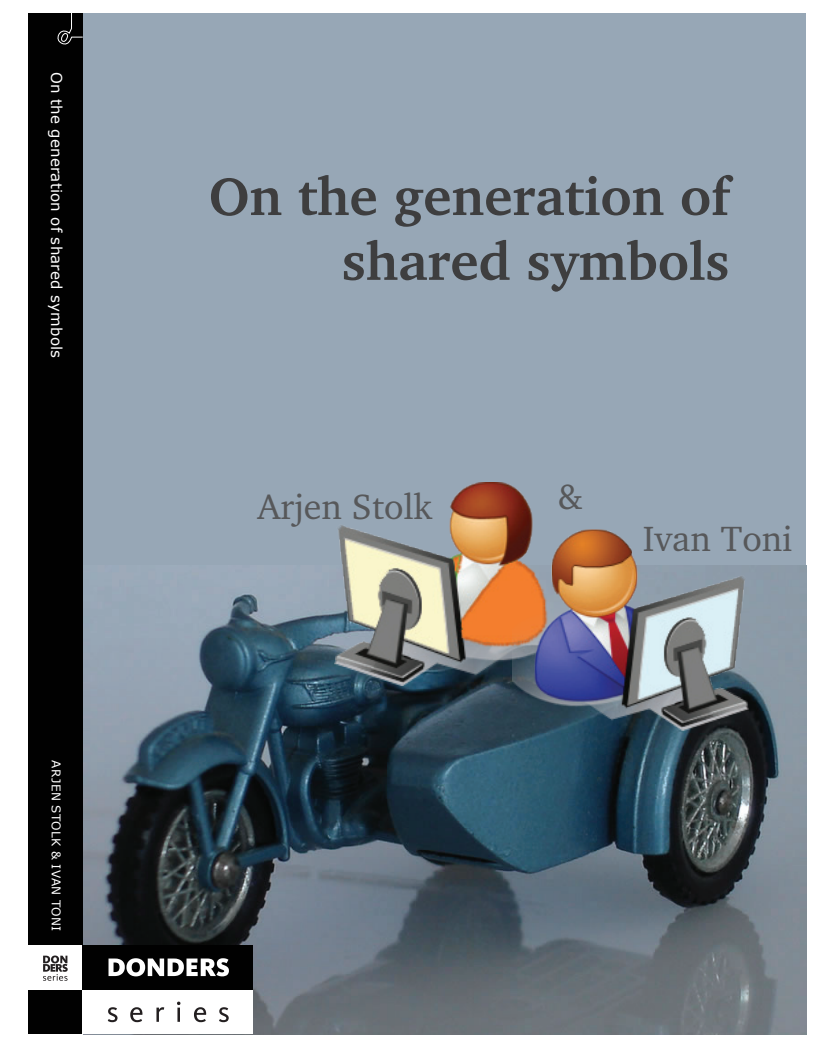

\section{About the author}

Arjen Stolk was born on the 31 $1^{\text {st }}$ of October 1982 in Arnhem, the Netherlands. He studied Human Movement Sciences at the University of Groningen. In this context, he wrote his bachelor's thesis in 2005 under the supervision of At Hof, on muscle activity patterns in human gait, as obtained with electromyography (EMG). In 2007 he wrote his master's thesis under the supervision of Bert Otten, Ritske de Jong and Mark Span, on the role of motor imagery in brain-computer interfacing, as assessed with electroencephalography (EEG). He then applied this knowledge at the University of Twente and the Medisch Spectrum Twente, to develop a brain-computer interface for the purpose of stroke rehabilitation. In 2009 he decided to switch to the field of Cognitive Neuroscience, and moved to the Donders Institute for Brain, Cognition and Behaviour. Here, he investigated the brain mechanisms underlying human communicative abilities under the supervision of Ivan Toni, and he became a member of the core team responsible for developing the FieldTrip open source software package for acquisition and analysis of electrophysiological data. At the Donders, he wrote this thesis, based on findings obained with magnetoencephalography (MEG), functional magnetic resonance imaging (fMRI), transcranial magnetic stimulation (TMS), and behavioral experiments involving patient and child populations. Since 2013 he is a postdoctoral researcher at the Donders Institute, using pharmacological intervention and electrophysiology to further unravel the brain mechanisms underlying human communication.

Alternative thesis front cover. 


\section{Publications}

Blokpoel M, van Kesteren M, Stolk A, Haselager P, Toni I, van Rooij I (2012). Recipient design in human communication: simple heuristics or perspective taking? Front Hum Neurosci 6

Stolk A, Todorovic A, Schoffelen JM, Oostenveld R (2013). Online and offline tools for head movement compensation in MEG. Neuroimage 68: 39-48

Stolk A, Verhagen L, Schoffelen JM, Oostenveld R, Blokpoel M, Hagoort P, van Rooij I, Toni I (2013). Neural mechanisms of communicative innovation. Proc Natl Acad Sci U S A 110: 14574-14579

Stolk A, Hunnius S, Bekkering H, Toni I (2013). Early social experience predicts referential communicative adjustments in five-year-old children. PLoS One 8

Stolk A, Noordzij ML, Volman I, Verhagen L, Overeem S, van Elswijk G, Bloem B, Hagoort P, Toni I (2014). Understanding communicative actions: a repetitive TMS study. Cortex 51: 25-34.

Stolk A (2014). In sync: metaphor, mechanism or marker of mutual understanding? J Neurosci 34(16): 5397-8

Stolk A, Blokpoel M, van Rooij I, Toni I (2014). On the generation of shared symbols. In R. Willems (Ed.), Towards a cognitive neuroscience of natural language use. Cambridge University Press, in press

\section{In preparation}

Brinkman L, Stolk A, Dijkerman HC, de Lange F, Toni I. Distinct roles for alphaand beta-band oscillations during the construction of goal-directed action plans.

Stolk A, Noordzij ML, Verhagen L, Volman I, Schoffelen JM, Oostenveld R, Hagoort P, Toni I. How minds meet: cerebral coherence between communicators marks the emergence of meaning.

Stolk A, D’Imperio D, di Pellegrino G, Toni I. Altered communicative adjustments following ventromedial prefrontal lesions.

Hartstra E, Stolk A, Mars R, Toni I, Ullsperger M. Temporal dynamics of posterror adjustments: an MEG study.

Verhagen L, Murillo Oosterwijk A, Stolk A, de Boer M, Toni I. Kinematics of communicative pointing.

de Boer M, Stolk A, Kokal I, Blokpoel M, Roelofs K, van Rooij I, Toni I. When reality and beliefs differ, oxytocin biases communicative choices towards reality.

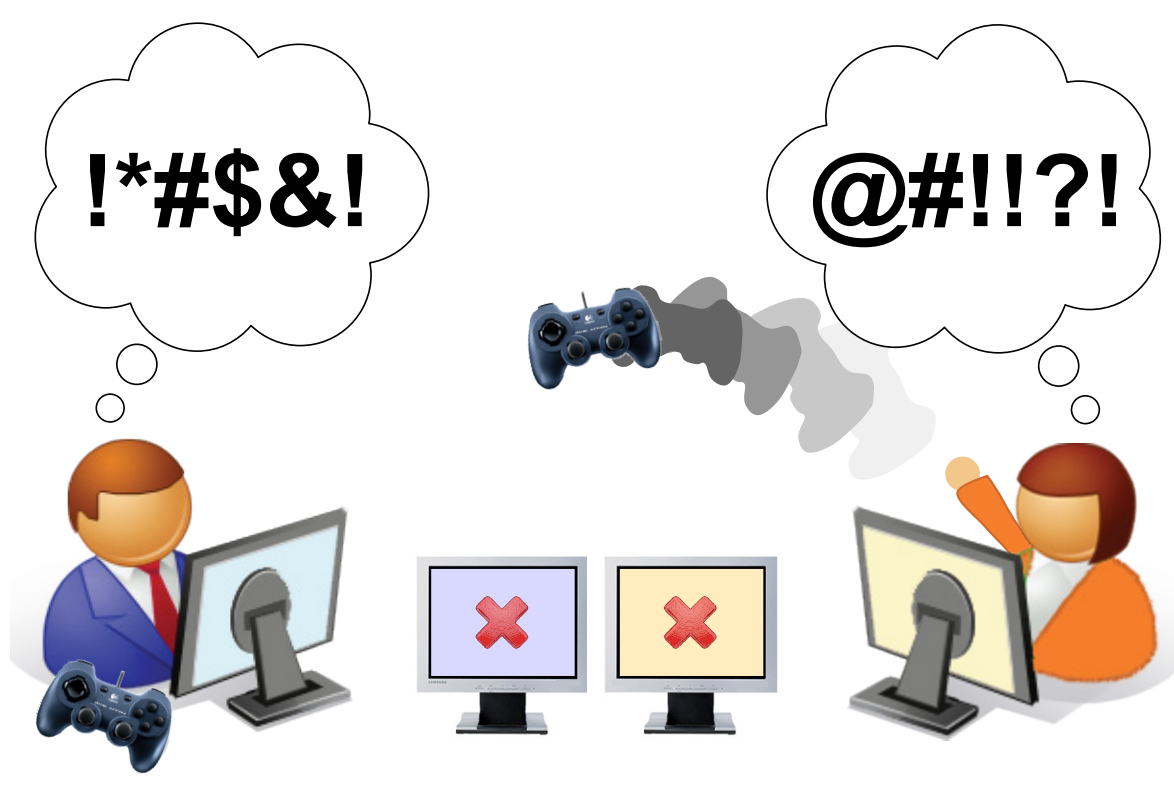




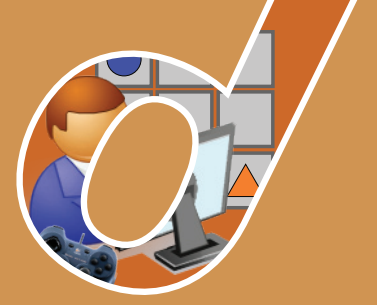

ISBN 978-94-62592-52-0 